\title{
Estrutura uniforme de espaços de Banach
}

\author{
João Gabriel Vitor de Carvalho
}

DisSERTAÇÃO APRESENTADA

$\mathrm{AO}$

Instituto DE MATEMÁtica e EstatísticA

$\mathrm{DA}$

Universidade DE SÃo PAUlo

PARA

OBTENÇÃO DO TÍTULO

$\mathrm{DE}$

Mestre em CiênCIAS

Programa: Matemática

Orientador: Prof. Dr. Wilson Albeiro Cuellar Carrera

Durante o desenvolvimento deste trabalho o autor recebeu auxílio financeiro da FAPESP, processo 2018/25569-0

São Paulo, fevereiro de 2021 


\section{Estrutura uniforme de Espaços de Banach}

Esta versão da dissertação contém as correções e alterações sugeridas pela Comissão Julgadora durante a defesa da versão original do trabalho, realizada em 19/03/2021. Uma cópia da versão original está disponível no

Instituto de Matemática e Estatística da Universidade de São Paulo.

Comissão Julgadora:

- Prof. Dr. Wilson Albeiro Cuellar Carrera (orientador) - IME-USP

- Prof. Dr. Thiago Grando - Unicentro

- Prof. Dr. Willian Hans Goes Corrêa - ICMC- USP 


\section{Agradecimentos}

Agradeço ao meu pai Galdino e aos meus irmãos Matheus e Lucas por todo apoio que me deram não só durante o mestrado como em toda minha vida. Agradeço e dedico esse texto à minha mãe Joelma (in memoriam), que sem dúvidas foi o ser humano mais incrível que conheci e com quem aprendi lições que levarei para toda vida.

Agradeço aos meus amigos Tiago, Hilário, Ana, Flávia e Giovani, que fizeram parte de várias fases da minha vida e com quem eu pude contar nos momentos mais difíceis.

Agradeço a meu orientador Wilson Cuellar, que foi sempre solícito para qualquer problema dentro e fora do mestrado. Agradeço também aos professores da graduação Thiago Castilho, Pedro Kauffman, Tiago Macedo e Leandro Candido, que lecionaram disciplinas que marcaram minha trajetória e sem dúvidas fizeram com que eu me apaixonasse ainda mais pela matemática.

Finalmente, agradeço a FAPESP pelo auxílio financeiro para o processo 2018/25569-0. 


\section{Resumo}

CARVAlHO, J. G. V. Estrutura uniforme de espaços de Banach. 2021. 107 f. Dissertação (Mestrado) - Instituto de Matemática e Estatística, Universidade de São Paulo, São Paulo, 2021.

Um problema comum em matemática é determinar quando dois objetos têm uma mesma estrutura, por exemplo, determinar quando dois espaços topológicos são homeomorfos. Em Análise Funcional, sabe-se que dois espaços de Banach isomorfos têm a mesma estrutura de espaço vetorial topológico, mas é possível que outras classes de funções entre espaços de Banach também preservem esta estrutura. Um resultado forte nesta linha é o clássico teorema de Mazur-Ulam, que afirma que uma isometria sobrejetora entre espaços de Banach reais é uma função afim, logo isometrias também preservam a estrutura linear de espaços de Banach reais. Este resultado motiva o estudo sobre o quanto da estrutura linear é preservada por outras classes de funções não lineares. No capítulo 3 trabalha-se com isomorfismos e mergulhos Lipschitz entre espaços de Banach. São desenvolvidos conceitos como conjuntos Haar-nulos, diferenciabilidade de Gâteaux e propriedade de Radon-Nikodým. É mostrado um resultado de Heinrich e Mankiewicz que diz que se $X$ e $Y$ são espaços de Banach e $Y$ tem a propriedade de Radon-Nikodým, entao todo mergulho Lipschitz $f: X \rightarrow Y$ pode ser linearizado através da derivada de Gâteaux. Este resultado irá abrir portas para vários outros resultados a respeito de isomorfismos Lipschitz e propriedades estáveis sob isomorfismos Lipschitz. Mostra-se por exemplo, que para $1<p<\infty$, todo espaço de Banach Lipschitz isomorfo a um espaço $L_{p}$ é também linearmente isomorfo a $L_{p}$. No capítulo 4 trabalha-se com os homeomorfismos uniformes. Resultados como o princípio de Gorelik e da teoria de Ramsey possibilitam estimativas relacionadas aos homeomorfismos uniformes que restringirão quando dois espaços de Banach podem ser uniformemente homeomorfos. Pode-se então provar o clássico teorema de Johnson, Lindenstrauss e Schechtman à respeito da estrutura uniforme dos espaços $\ell_{p}$ 's: se $1<p<\infty$ e $X$ é um espaço de Banach uniformemente homeomorfo a $\ell_{p}$, então $X$ é linearmente isomorfo a $\ell_{p}$. Enfim, prova-se o mesmo para os espaços $\ell_{p} \oplus \ell_{q}$, onde $1<p<q<2$ ou $2<p<q<\infty$ e depois para os espaços $\ell_{p} \oplus \ell_{q}$, onde $1<p<2<q<\infty$. Aquele estabelecido por Johnson, Lindenstrauss e Schechtman e este estabelecido por Kalton e Randrianarivony.

Palavras-chave: espaços de Banach, homeomorfismos uniformes, funções Lipschitz. 


\section{Abstract}

CARVAlHO, J. G. V. Uniform structure of Banach Spaces. 2021. 107 f. Dissertação (Mestrado) - Instituto de Matemática e Estatística, Universidade de São Paulo, São Paulo, 2021.

A common problem in mathematics is to determine when two objects have the same structure, for instance, determine when two topological spaces are homeomorphic. In Functional Analysis, it is known that two isomorphic Banach spaces have the same structure as topological vector spaces, but it is possible that others classes of functions preserve this structure. A strong result in this way is the classic Mazur-Ulam theorem, that asserts that a surjective isometry between real Banach spaces is an affine function, hence isometries also preserve the linear structure of real Banach spaces. In chapter 3 we deal with Lipschitz isomorphisms and embeddings. Concepts such as Haar null sets, Gâteaux differentiability and Radon-Nikodým property are developed. We show a result of Heinrich and Mankiewicz, that says that if $X$ and $Y$ are Banach spaces and $Y$ has the Radon-Nikodým property, then every Lipschtiz embedding $f: X \rightarrow Y$ can be linearized through the Gâteaux derivative. This result will make way to many others results concerning Lipschitz isomorphisms and stable properties under Lipschitz isomorphisms. It is shown, for instance, that for $1<p<\infty$, every Banach space Lipschitz isomorphic to a $L_{p}$ space is also linearly isomorphic to $L_{p}$. In chapter 4, we deal with uniform homeomorphisms. Results such as the Gorelik principle and results from Ramsey theory enable bounds related to the uniform homeomorphisms that will restrict when two Banach spaces can be uniform homeomorphic. Then, we can prove the classic theorem of Johnson, Lindenstrauss and Schechtman concerning the uniform structure of the spaces $\ell_{p}$ 's: if $1<p<\infty$ and $X$ is a Banach space uniformly homeomorphic to $\ell_{p}$, then $X$ is linearly isomorphic to $\ell_{p}$. In the end, it is proved the same for the spaces $\ell_{p} \oplus \ell_{q}$, where $1<p<q<2$ or $2<p<q<\infty$ and afterwards for the spaces $\ell_{p} \oplus \ell_{q}$, where $1<p<2<q<\infty$. The first one was established by Johnson, Lindenstrauss and Schechtman and the second one was established by Kalton and Randrianarivony.

Keywords: Banach spaces, uniform homeomorphisms, Lipschitz functions. 


\section{Sumário}

$\begin{array}{ll}\text { Lista de Abreviaturas } & \text { ix }\end{array}$

Lista de Símbolos $\quad$ xi

1 INTRODUÇÃOO 1

2 Preliminares $\quad 3$

2.1 Medida e Integração . . . . . . . . . . . . . . . . . . . . . . . 3

2.2 Probabilidade . . . . . . . . . . . . . . . . . 8

2.3 Análise Funcional . . . . . . . . . . . . . . . . . . . . . . . . . . . 14

2.3 .1 Conceitos e teoremas básicos . . . . . . . . . . . . . . . . . . 14

2.3 .2 Redes . . . . . . . . . . . . . . . . . . . . . 18

2.3.3 Ultrafiltros e Ultraprodutos . . . . . . . . . . . . . . . . . . 20

2.3.4 Propriedades locais de Espaços de Banach . . . . . . . . . . . . 24

3 FunÇões Lipschitz Entre espaÇos De Banach 31

3.1 Conjuntos Haar Nulos . . . . . . . . . . . . . . . . . . . . . . . . . 32

3.2 Integral de Bochner . . . . . . . . . . . . . . . . . . . . 38

3.3 Teorema de Rademacher para espaços de Banach . . . . . . . . . . . . . . . 44

3.4 Teorema de Rademacher fraco estrela . . . . . . . . . . . . . . . . . . . . 56

3.5 Isomorfismos Lipschitz entre espaços Reflexivos . . . . . . . . . . . . . . 62

4 Homeomorfismos Uniformes ENTRE ESPAÇOS DE BANACH 71

4.1 Definições Básicas . . . . . . . . . . . . . . . . . . . . . . 71

4.2 Pontos médio aproximados . . . . . . . . . . . . . . . . . . 77

4.3 Princípio de Gorelik . . . . . . . . . . . . . . . . . . . . . . . 80

4.4 Grafos Métricos . . . . . . . . . . . . . . . . . . . 81

4.5 Estrutura Uniforme dos espaços $\ell_{p} \ldots \ldots \ldots$. . . . . . . . . . . . 86

4.6 Estrutura Uniforme dos espaços $\ell_{p} \oplus \ell_{q} \ldots \ldots \ldots$. . . . . . . . . 95

5 Problemas em Aberto 101

$\begin{array}{lll}\text { A Teorema de Mazur Ulam } & 103\end{array}$ 
viii SUMÁRIO

Referências Bibliográficas

105

Índice Remissivo 


\section{Lista de Abreviaturas}

RNP Propriedade de Radon-Nikodým (Radon-Nikodým Property)

CLC Grosseira Lipschitz Contínua (Coarse Lipschitz Continuous) 


\section{Lista de Símbolos}

$\mathcal{P}(X) \quad$ Conjunto das partes de um conjunto $X$;

$\mathbb{N} \quad\{1,2,3, \ldots\} ;$

$\mathbb{Q} \quad$ Conjunto dos números racionais;

$\mathbb{R} \quad$ Conjunto dos números reais;

$[-\infty, \infty]$ Conjunto $\mathbb{R} \cup\{-\infty\} \cup\{+\infty\} ;$

$\mathbb{C} \quad$ Conjunto dos números complexos;

$\mathbb{F} \quad$ Corpo dos números reais ou complexos;

$[A] \quad$ Subespaço fechado gerado por um subconjunto $A$ de um espaço vetorial $V$;

$\ell_{p} \quad$ Espaço normado das sequências $\left(x_{n}\right)_{n \in \mathbb{N}}$ com valores em $\mathbb{F}$ tais que $\sum_{n \in \mathbb{N}}\left|x_{n}\right|^{p}<\infty$, onde $1 \leq p<\infty$ e cuja norma é dada por $\left\|\left(x_{n}\right)_{n \in \mathbb{N}}\right\|_{p}=$ $\left(\sum_{n \in \mathbb{N}}\left|x_{n}\right|^{p}\right)^{1 / p}$ para cada $\left(x_{n}\right)_{n \in \mathbb{N}} \in \ell_{p}$

$\ell_{\infty} \quad$ Espaço normado das sequências $\left(x_{n}\right)_{n \in \mathbb{N}}$ com valores em $\mathbb{F}$ tais que $\sup _{n \in \mathbb{N}}\left|x_{n}\right|<\infty$, cuja norma é dada por $\left\|\left(x_{n}\right)_{n \in \mathbb{N}}\right\|_{\infty}=\sup _{n \in \mathbb{N}}\left|x_{n}\right|$ para cada $\left(x_{n}\right)_{n \in \mathbb{N}} \in \ell_{\infty}$

$c_{0} \quad$ Subespaço de $\ell_{\infty}$ das sequências $\left(x_{n}\right)_{n \in \mathbb{N}}$ em $\mathbb{F}$ tais que $\lim _{n \in \mathbb{N}} x_{n}=0$;

$L_{p} \quad$ Espaço das funções $f:[0,1] \rightarrow \mathbb{R}$ Lebesgue mensuráveis tais que $\int_{0}^{1}|f(x)|^{p} d x<\infty$, identificando funções $f$ e $g$ tais que $f=g$ quase sempre com respeito à medida de Lebesgue e cuja a norma é dada por $\|f\|_{p}=$ $\left(\int_{0}^{1}|f(x)|^{p} d x\right)^{1 / p}$

$\mathcal{L}(X, Y) \quad\{T: X \rightarrow Y: T$ é linear e contínua $\}$, onde $X$ e $Y$ são espaços normados;

$B_{X}^{d}(x ; r) \quad\{y \in X: d(x, y)<r\}$, onde $(X, d)$ é um espaço métrico e $r>0$;

$B_{X} \quad\{y \in X:\|y\| \leq 1\}$, onde $X$ é um espaço normado;

$d(x, A) \quad \inf \{d(x, a): a \in A\}$, onde $(X, d)$ é um espaço métrico e $A \subset X$;

$\operatorname{diam}(A) \sup \{d(x, y): x, y \in A\}$, onde $(X, d)$ é um espaço métrico e $A \subset X$;

$\operatorname{Bor}(X) \quad\{A \subset X: A$ é boreliano $\}$, onde $X$ é um espaço topológico;

$\operatorname{Pr}(X) \quad$ Conjunto de todas medidas de probabilidade em $(X, \operatorname{Bor}(X))$, onde $X$ é um espaço topológico;

$\operatorname{Lip}(f) \quad \sup \left\{\frac{d_{Y}(f(x), f(y))}{d_{X}(x, y)}: x, y \in X\right.$ e $\left.x \neq y\right\}$, onde $f: X \rightarrow Y$ é uma função lipschtziana entre espaços métricos $\left(X, d_{X}\right)$ e $\left(Y, d_{Y}\right)$; 
$\mathcal{U} \quad$ Ultrafiltro em $\mathbb{N}$;

$X_{\mathcal{U}} \quad$ Ultraproduto de um espaço de Banach $X$ por um ultrafiltro $\mathcal{U}$;

$\operatorname{Mid}(x, y, \delta) \quad\{z \in X: \max (\|x-z\|,\|y-z\|) \leq(1+\delta)\|x-y\| / 2\}$, onde $X$ é um espaço normado, $x, y \in X$ e $\delta>0$;

$G_{k}(\mathbb{M}) \quad$ Espaço métrico de todos subconjuntos de $\mathbb{M}$ com tamanho $k$ equipado com a métrica $d\left(\left(n_{1}, \ldots, n_{k}\right),\left(m_{1}, \ldots, m_{k}\right)\right)=\left|\left\{i: n_{i} \neq m_{i}\right\}\right|$, onde $\left\{n_{1}, \ldots, n_{k}\right\}$, $\left\{m_{1}, \ldots, m_{k}\right\} \in G_{k}(\mathbb{M})$, onde as $k$-tuplas estão ordenadas de maneira crescente e $\mathbb{M}$ é um subconjunto infinito de $\mathbb{N}$;

$\omega_{f}(t) \quad \sup \left\{d_{2}(f(x), f(y)): x, y \in M_{1}\right.$ e $\left.d_{1}(x, y) \leq t\right\}$ para cada $t>0$, onde $f: M_{1} \rightarrow M_{2}$ é uma função entre espaços métricos $M_{1}$ e $M_{2}$;

$\operatorname{Lip}_{\infty}(f) \quad \limsup _{t \rightarrow \infty} \omega_{f}(t) / t$, onde $f: M_{1} \rightarrow M_{2}$ é uma função entre espaços métricos $M_{1}$ e $M_{2}$ tal que $\limsup _{t \rightarrow \infty} \omega_{f}(t) / t<\infty$;

$K_{f}(t) \quad \sup \left\{\frac{d_{2}(f(x), f(y))}{\max \left\{t, d_{1}(x, y)\right\}}: x, y \in M_{1}\right.$ e $\left.x \neq y\right\}$, onde $f: M_{1} \rightarrow M_{2}$ é uma função entre espaços métricos $M_{1}$ e $M_{2}$; 


\section{Capítulo 1}

\section{INTRODUÇÃO}

Um problema comum em matemática é determinar quando dois objetos têm uma mesma estrutura; por exemplo, determinar quando dois grupos são isomorfos ou quando dois espaços topológicos são homeomorfos. Em Análise Funcional, sabemos que dois espaços de Banach isomorfos têm a mesma estrutura de espaço vetorial topológico, mas podemos nos perguntar o quanto desta estrutura é preservado por funções não-lineares. Temos por exemplo, o clássico teorema de Mazur-Ulam (veja o apêndice para uma demonstração).

Teorema 1.0.1 (Mazur-Ulam). Sejam $X, Y$ espaços de Banach reais e $f: X \rightarrow Y$ uma isometria sobrejetora de $X$ em $Y$. Então $f$ é uma função afim.

Logo, a estrutura de espaço métrico de um espaço de Banach real determina toda sua estrutura linear. No outro extremo, temos um resultado de [Kad67] e [Tor81]. Lembramos que o caráter de densidade de um espaço topológico é a menor cardinalidade de um conjunto denso.

Teorema 1.0.2 (Kadets, Toruńczyk). Sejam X e Y espaços de Banach de dimensão infinita com o mesmo caráter de densidade. Então $X$ e $Y$ são homeomorfos.

Então, um homeomorfismo não diz nada sobre a estrutura linear dos espaços de Banach. Vamos considerar então algumas classes de funções não-lineares que sejam mais abrangentes que as isometrias e menos abundantes que os homeomorfismos, como por exemplo os homeomorfismos coarse, os homeomorfismos uniformes e os isomorfismos Lipschitz. Veremos que para alguns espaços clássicos, como os espaços $\ell_{p}$ 's e os espaços $L_{p}$ 's, veremos alguns resultados positivos a respeito de sua estrutura não-linear.

No segundo capítulo vamos introduzir boa parte dos conceitos que iremos usar ao decorrer do texto. Em particular, vamos relembrar os principais resultados da teoria de medida e todos os conceitos necessários da teoria de probabilidade. Estes conceitos serão fundamentais no desenvolvimento do capítulo 3. Também relembraremos alguns conceitos de análise funcional e desenvolver o básico sobre redes e ultraprodutos. Para finalizar, vamos falar sobre propriedades locais de espaços de Banach, isto é, propriedades de subespaços de dimensão 
finita. Estas propriedades serão importantes no capítulo 4 pois veremos que elas são preservadas por homeomorfismos uniformes.

No capítulo seguinte vamos trabalhar com funções lipschitzianas entre espaços de Banach. Começaremos demonstrando uma generalização do clássico teorema de Rademacher.

Teorema 1.0.3 (Rademacher). Seja $f: \mathbb{R}^{n} \rightarrow \mathbb{R}^{m}$ uma função Lipschitz. Então $f$ é diferenciável quase sempre com respeito à medida de Lebesgue.

Para isso, desenvolveremos a noção de conjuntos Haar Nulos, que será responsável por traduzir o conceito de subconjuntos de $\mathbb{R}^{n}$ com medida de Lebesgue nula para subconjuntos de um espaço de Banach $X$. Também vamos desenvolver a integração de Bochner, responsável por integrar funções cujo codomínio é um espaço de Banach, e a propriedade de RadonNikodým. Com isso desenvolvido, mostraremos um resultado de [HM82] que diz que se $X$ e $Y$ são espaços de Banach, $Y$ tem a propriedade de Radon-Nikodým e $f: X \rightarrow Y$ é um mergulho Lipschitz, isto é, uma função para a qual existem $A, B>0$ tais que

$$
A\|x-y\| \leq\|f(x)-f(y)\| \leq B\|x-y\| \text { para cada } x, y \in X
$$

então podemos linearizar este mergulho Lipschtz através da derivada de Gâteaux.

Em seguida, vamos trabalhar com mergulhos Lipschitz da forma $f: X \rightarrow Z^{*}$, onde $X$ e $Z$ são espaços de Banach com $X$ separável. Introduziremos o conceito de fraca*-diferenciabilidade e mostrar um outro resultado de [HM82] que nos permite linearizar tais funções, mas dessa vez sem ser necessária a propriedade de Radon-Nikodým.

Por fim, mostraremos que isomorfismos Lipschitz $f: X \rightarrow Y$ entre espaços de Banach reflexivos $X$ e $Y$ implicam que $X$ é isomorfo a um subespaço complementado de $Y$ e viceversa. Conseguimos então que $X$ e $Y$ são isomorfos se $X$ e $Y$ satisfazem a decomposição de Pełczyński, que será abordada durante o capítulo.

No quarto capítulo, vamos trabalhar com homeomorfismos uniformes. Para conseguir estimativas através dessa classe de funções, vamos trabalhar com dois conceitos diferentes. O primeiro é o princípio de Gorelik, que nos diz que um homeomorfismo uniforme não pode enviar bolas grandes de um espaço de codimensão finita em uma vizinhança pequena de um espaço de codimensão infinita. Junto à noção de pontos médio aproximados, vamos conseguir aplicar o princípio para espaços como $\ell_{p}, \ell_{p} \oplus \ell_{q}$ e $c_{0}$. O segundo conceito é o estudo de grafos métricos em $\mathbb{N}$. Junto à teoria de Ramsey vamos demonstrar algumas estimativas de funções cujo domínio é um grafo métrico em um subconjunto infinito de $\mathbb{N}$. Vamos provar então o clássico resultado de [JLS96] que nos diz que espaços $\ell_{p}$ 's, para $1<p<\infty$, são determinados por sua estrutura uniforme, isto é, todo espaço de Banach uniformemente homeomorfo a $\ell_{p}$ com $1<p<\infty$ é também isomorfo a $\ell_{p}$. Depois vamos mostrar que o mesmo vale para as somas $\ell_{p} \oplus \ell_{q}$. Este resultado foi provado em [JLS96] para $1<p<q<2$ e $2<p<q<\infty$ e o caso $1<p<2<q<\infty$ foi provado em [Kal08] utilizando o conceito de grafos métricos. 


\section{Capítulo 2}

\section{PRELIMINARES}

\subsection{Medida e Integração}

Nesta seção iremos relembrar conceitos e resultados relacionados à teoria de medida que usaremos ao decorrer do texto. Como referência principal indicamos [Rud70] e [Dur13]. Vamos fixar um espaço de medida arbitrário $(\Omega, \mathcal{F}, \mu)$ ao longo da seção. Fixamos $\operatorname{Bor}\left(\mathbb{R}^{k}\right)$ como sendo a $\sigma$-álgebra dos borelianos, isto é, a menor $\sigma$-álgebra contendo os abertos de $\mathbb{R}^{k}$. Também fixamos $\left(\mathbb{R}^{k}, \mathfrak{M}_{\mathbb{R}^{k}}, m_{\mathbb{R}^{k}}\right)$ como sendo o espaço de medida de Lebesgue em $\mathbb{R}^{k}$.

Definição 2.1.1. Sejam $X$ um conjunto, $A \subset X e\left(A_{n}\right)_{n \in \mathbb{N}}$ uma sequência de subconjuntos de $X$. Dizemos que $A_{n} \nearrow A$ se $A_{n} \subset A_{n+1}$ para todo $n \in \mathbb{N}$ e $A=\bigcup_{n \in \mathbb{N}} A_{n}$.

Proposição 2.1.2. Sejam $A \in \mathcal{F} e\left(A_{n}\right)_{n \in \mathbb{N}}$ uma sequência de conjuntos de $\mathcal{F}$ tal que $A_{n} \nearrow$ A. Então $\lim _{n \in \mathbb{N}} \mu\left(A_{n}\right)=\mu(A)$.

Demonstração. Ver [Rud70] Teorema 1.19-(d), página 16.

Definição 2.1.3. Seja $X$ um conjunto e $\mathcal{A} \subset \mathcal{P}(X)$. Definimos $\sigma(\mathcal{A})$ como sendo a $\sigma$-álgebra gerada por $\mathcal{A}$, isto é, a menor $\sigma$-álgebra contendo $\mathcal{A}$.

Definição 2.1.4. Seja $X$ um conjunto. Dizemos que $\mathcal{A} \subset \mathcal{P}(X)$ é

1. um $\pi$-sistema se $A, B \in \mathcal{A}$ implica $A \cap B \in \mathcal{A}$.

2. um $\lambda$-sistema se:

(a) $X \in \mathcal{A}$;

(b) $A, B \in \mathcal{A}$ e $A \subset B$ implicam $B \backslash A \in \mathcal{A}$;

(c) $A_{n} \in \mathcal{A}$ e $A_{n} \nearrow A$ implicam $A \in \mathcal{A}$.

Teorema 2.1.5 (Lema $\pi$ - $\lambda$ ). Sejam $\mathcal{A}$ um $\pi$-sistema e $\mathcal{B}$ um $\lambda$-sistema tal que $\mathcal{A} \subset \mathcal{B}$. Então $\sigma(\mathcal{A}) \subset \mathcal{B}$.

Demonstração. Ver [Dur13] Teorema A.1.4, página 344. 
Lembramos que uma função $f: \Omega \rightarrow[-\infty, \infty]$ é dita mensurável se $f^{-1}[A] \in \mathcal{F}$ para cada boreliano $A$ de $[-\infty, \infty]$. A proposição a seguir é uma condição suficiente para que uma função seja mensurável.

Proposição 2.1.6. Seja $f: \Omega \rightarrow[-\infty, \infty]$ uma função tal que $f^{-1}[(\alpha, \infty]] \in \mathcal{F}$ para cada $\alpha \in \mathbb{R}$. Então $f$ é uma função mensurável.

Demonstração. Indicamos [Rud70] Teorema 1.12.

Definição 2.1.7. Seja $P$ uma propriedade para a qual um ponto $x \in \Omega$ pode ter ou não. Dizemos que $P$ vale quase sempre com respeito ao espaço de medida $(\Omega, \mathcal{F}, \mu)$ se existe um conjunto $A \in \mathcal{F}$ tal que $\mu(A)=0$ e $P$ vale para todo ponto de $\Omega \backslash A$.

Estamos particularmente interessados no caso em que $f: \Omega \rightarrow \mathbb{C}$ é uma função mensurável e $P$ é a propriedade $f(x)=0$. Então dizemos que $f=0$ quase sempre se existe um conjunto $A \in \mathcal{F}$ tal que $\mu(A)=0$ e $f(x)=0$ para todo $x \in \Omega \backslash A$.

Proposição 2.1.8. Sejam $X$ espaço topológico, $(Y, d)$ espaço métrico completo e $\left(f_{n}\right)_{n \in \mathbb{N}}$ uma sequência de funções $f_{n}: X \rightarrow Y$ contínuas. Então o conjunto

$$
S=\left\{x \in X: \text { existe o limite } \lim _{n \in \mathbb{N}} f_{n}(x)\right\}
$$

é boreliano.

Demonstração. De fato, podemos escrever

$$
\begin{aligned}
S & =\bigcap_{n \in \mathbb{N}} \bigcup_{k \in \mathbb{N}}\left\{x \in X: d\left(f_{k_{1}}(x), f_{k_{2}}(x)\right)<\frac{1}{n} \text { para cada } k_{1}, k_{2} \geq k\right\} \\
& =\bigcap_{n} \bigcup_{k} \bigcap_{k_{1}, k_{2} \geq k}\left\{x \in X: d\left(f_{k_{1}}(x), f_{k_{2}}(x)\right)<\frac{1}{n}\right\} .
\end{aligned}
$$

Como o conjunto $\left\{x \in X: d\left(f_{k_{1}}(x), f_{k_{2}}(x)\right)<\frac{1}{n}\right\}$ é aberto para cada $k_{1}, k_{2} \in \mathbb{N}$ e $n \in \mathbb{N}$, o resultado segue.

Lema 2.1.9 (Fatou). Seja $\left(f_{n}\right)_{n \in \mathbb{N}}$ uma sequência de funções $f_{n}: \Omega \rightarrow[0, \infty]$ mensuráveis. Então

$$
\int_{\Omega} \liminf _{n \in \mathbb{N}} f_{n} d \mu \leq \liminf _{n \in \mathbb{N}} \int_{\Omega} f_{n} d \mu
$$

Demonstração. Ver [Rud70] Proposição 1.28, página 22.

Definição 2.1.10. Sejam $1 \leq p<\infty$ e $f: \Omega \rightarrow \mathbb{C}$ uma função mensurável. Definimos

$$
\|f\|_{p}=\left(\int_{\Omega}|f|^{p} d \mu\right)^{1 / p} \in[0, \infty] .
$$

Chamamos de espaço $L^{p}(\mu)$ o espaço de todas as funções $f: \Omega \rightarrow \mathbb{C}$ mensuráveis para as quais o valor $\|f\|_{p}$ é finito. 
Teorema 2.1.11 (Desigualdade de Minkowski). Sejam $1 \leq p<\infty, f: \Omega \rightarrow[0, \infty] e$ $g: \Omega \rightarrow[0, \infty]$ mensuráveis. Então

$$
\left(\int_{\Omega}(f+g)^{p} d \mu\right)^{1 / p} \leq\left(\int_{\Omega} f^{p} d \mu\right)^{1 / p}+\left(\int_{\Omega} g^{p} d \mu\right)^{1 / p} .
$$

Demonstração. Ver [Rud70] Teorema 3.5-(2), página 62.

Embora $\|\cdot\|_{p}$ satisfaça quase todas as propriedades de uma norma, temos que $\|f\|_{p}=0$ para todo $f=0$ quase sempre. Definimos então uma relação de equivalência $\equiv \operatorname{em~} L^{p}(\mu)$ dada por $f \equiv g$ se e somente se $f=g$ quase sempre.

Definição 2.1.12. Definimos o espaço vetorial $L_{p}(\mu)$ como sendo o quociente $L^{p}(\mu) / \equiv$. Dada $\bar{f} \in L_{p}(\mu)$, definimos o valor $\|\bar{f}\|_{p}$ como sendo $\|f\|_{p}$, onde $f \in \bar{f}$.

Proposição 2.1.13. A função $\|\cdot\|_{p}: L_{p}(\mu) \rightarrow[0, \infty)$ definida por $\|\cdot\|_{p}(\bar{f})=\|\bar{f}\|_{p}$ é bem definida e é uma norma completa em $L_{p}(\mu)$.

Demonstração. Ver [Rud70] Teorema 3.9, página 65 e Teorema 3.11, página 66.

Para não sobrecarregar a notação, sempre que pudermos vamos trabalhar com funções representantes ao invés das classes de equivalência em $L_{p}(\mu)$.

No caso em que o espaço de medida é $([0,1], \mathfrak{M}, m)$, vamos chamar o espaço $L_{p}(m)$ de $L_{p}[0,1]$ ou simplesmente de $L_{p}$, desde que esteja claro sobre qual espaço estamos nos referindo.

O resultado a seguir é uma propriedade de continuidade interessante do espaço $L_{1}$ e será utilizado no capítulo 3.

Teorema 2.1.14. Sejam $f \in L_{1}\left(\mathbb{R}^{n}\right)$. Então para quase todo $u \in \mathbb{R}^{n}$, vale

$$
\lim _{r \rightarrow 0^{+}} \frac{1}{m_{\mathbb{R}^{n}}(B(u ; r))} \int_{B(u ; r)}|f(y)-f(u)| d y=0 .
$$

Demonstração. Veja [Rud70] Teorema 8.8.

Dados dois espaços mensuráveis $(\Omega, \mathcal{F})$ e $(\Psi, \mathcal{G})$, denotamos por $(\Omega \times \Psi, \mathcal{F} \times \mathcal{G})$ o espaço mensurável produto. Se $\mu$ é uma medida em $(\Omega, \mathcal{F})$ e $\nu$ é uma medida em $(\Psi, \mathcal{G})$, denotamos por $\mu \otimes \nu$ a medida produto no espaço $(\Omega \times \Psi, \mathcal{F} \times \mathcal{G})$. A construção do espaço produto e da medida produto pode ser encontrada no capítulo 7 de [Rud70].

Definição 2.1.15. Dizemos que um espaço de medida $(\Omega, \mathcal{F}, \mu)$ é $\sigma$-finito se $\Omega=\bigcup_{n \in \mathbb{N}} A_{n}$, onde $A_{n} \in \mathcal{F}$ e $\mu\left(A_{n}\right)<\infty$ para cada $n \in \mathbb{N}$.

Definição 2.1.16. Dada uma função $f: \Omega \times \Psi \rightarrow \mathbb{C}$, definimos $f_{x}: \Psi \rightarrow \mathbb{C}$ por $f_{x}(y)=$ $f(x, y)$ para todo $x \in \Omega$ e analogamente, definimos $f_{y}: \Omega \rightarrow \mathbb{C}$ por $f_{y}(x)=f(x, y)$ para todo $y \in \Psi$. 
Proposição 2.1.17. Seja $f: \Omega \times \Psi \rightarrow \mathbb{C}$ mensurável. Então as funções $f_{x}$ e $f_{y}$ são mensuráveis com respeito aos espaços mensuráveis $(\Psi, \mathcal{G})$ e $(\Omega, \mathcal{F})$, respectivamente.

Demonstração. Ver [Rud70] Proposição 7.5, página 138.

Teorema 2.1.18 (Fubini para funções positivas). Sejam $(\Omega, \mathcal{F}, \mu)$ e $(\Psi, \mathcal{G}, \nu)$ espaços de medida $\sigma$-finitos. Seja $f: \Omega \times \Psi \rightarrow[0, \infty]$ uma função mensurável. Então as funções $x \mapsto$ $\int_{\Psi} f_{x} d \nu$ e $y \mapsto \int_{\Omega} f_{y} d \mu$ são mensuráveis e

$$
\int_{\Omega}\left(\int_{\Psi} f_{x} d \nu\right) d \mu=\int_{\Omega \times \Psi} f d(\mu \otimes \nu)=\int_{\Psi}\left(\int_{\Omega} f_{y} d \mu\right) d \nu .
$$

Demonstração. Ver [Rud70] Teorema 7.8 a), página 140.

Teorema 2.1.19 (Fubini para funções $\left.L_{1}\right)$. Sejam $(\Omega, \mathcal{F}, \mu)$ e $(\Psi, \mathcal{G}, \nu)$ espaços de medida $\sigma$-finito. Seja $f: \Omega \times \Psi \rightarrow \mathbb{C}$ uma função em $L_{1}(\mu \otimes \nu)$. Então $f_{x} \in L_{1}(\nu)$ para quase todo $x \in \Omega, f_{y} \in L_{1}(\mu)$ para quase todo $y \in \Psi$ e as funções definidas quase sempre $x \mapsto \int_{\Psi} f_{x} d \nu$ e $y \mapsto \int_{\Omega} f_{y} d \mu$ estão em $L_{1}(\mu)$ e $L_{1}(\nu)$, respectivamente. Por fim,

$$
\int_{\Omega}\left(\int_{\Psi} f_{x} d \nu\right) d \mu=\int_{\Omega \times \Psi} f d(\mu \otimes \nu)=\int_{\Psi}\left(\int_{\Omega} f_{y} d \mu\right) d \nu
$$

Demonstração. Ver [Rud70] Teorema 7.8 c), página 141.

Um caso particular e de nosso interesse é o caso em que os espaços de medida são $\left(\mathbb{R}^{n}, \mathfrak{M}_{\mathbb{R}^{n}}, m_{\mathbb{R}^{n}}\right)$ e $\left(\mathbb{R}^{m}, \mathfrak{M}_{\mathbb{R}^{\mathfrak{m}}}, m_{\mathbb{R}^{m}}\right)$. Vale ressaltar que a medida de Lebesgue $m_{\mathbb{R}^{n+m}}$ não é a medida produto $m_{\mathbb{R}^{n}} \otimes m_{\mathbb{R}^{m}}$. Porém segue do resultado 7.12 [Rud70] que os teoremas acima continuam valendo se trocarmos a medida produto $m_{\mathbb{R}^{n}} \otimes m_{\mathbb{R}^{m}}$ pela medida de Lebesgue $m_{\mathbb{R}^{n+m}}$. Em particular, temos

Teorema 2.1.20 (Fubini em $\mathbb{R}^{n} \times \mathbb{R}^{m}$ ). Seja $f: \mathbb{R}^{n} \times \mathbb{R}^{m} \rightarrow[0, \infty]$ uma função mensurável. Então as funções $x \mapsto \int_{\mathbb{R}^{n}} f_{x} d \lambda$ e $y \mapsto \int_{\mathbb{R}^{m}} f_{y} d \mu$ são mensuráveis $e$

$$
\int_{\mathbb{R}^{m}}\left(\int_{\mathbb{R}^{n}} f(x, y) d x\right) d y=\int_{\mathbb{R}^{n} \times \mathbb{R}^{m}} f d m_{\mathbb{R}^{n+m}}=\int_{\mathbb{R}^{n}}\left(\int_{\mathbb{R}^{m}} f(x, y) d y\right) d x .
$$

O resultado a seguir pode ser encontrado em contexto mais gerais, mas vamos enunciar uma versão simplificada que será o suficiente para o nosso propósito.

Teorema 2.1.21 (Mudança de Variável). Seja $A \subset \mathbb{R}^{n}$ um aberto e $g: A \rightarrow \mathbb{R}^{n}$ uma função injetora, derivável, cuja derivada é contínua e tal que $\operatorname{det} g^{\prime}(x) \neq 0$ para todo $x \in A$. Se $f: g(A) \rightarrow \mathbb{R}$ é integrável, então

$$
\int_{g(A)} f(y) d y=\int_{A}(f \circ g)(x)\left|\operatorname{det} g^{\prime}(x)\right| d x .
$$

Demonstração. Ver [Spi65] Teorema 3-13, página 67. 
Definição 2.1.22. Uma função $\nu: \mathcal{F} \rightarrow \mathbb{C}$ (respectivamente $\nu: \mathcal{F} \rightarrow \mathbb{R}$ ) é chamada de medida complexa (respectivamente medida real) se para cada sequência de mensuráveis $\left(E_{k}\right)_{k}$ satisfazendo $E_{k} \cap E_{l}=\emptyset$ para $k \neq l$, vale

$$
\nu\left(\bigcup_{k=1}^{\infty} E_{k}\right)=\sum_{k=1}^{\infty} \nu\left(E_{k}\right)
$$

Definição 2.1.23. Seja $\nu: \mathcal{F} \rightarrow \mathbb{C}$ uma medida complexa (real). Definimos a variação total de $\nu$ como sendo a função $|\nu|: \mathcal{F} \rightarrow[0,+\infty]$ dada por

$$
|\nu|(E)=\sup \left\{\sum_{k=1}^{\infty}\left|\nu\left(E_{k}\right)\right|: E=\bigcup_{k=0}^{\infty} E_{k} \text { e } E_{k} \cap E_{l}=\emptyset \text { para } k \neq l\right\}
$$

Teorema 2.1.24. A variação total $|\nu|$ de uma medida complexa (medida real) $\nu$ em $\mathcal{F}$ é uma medida positiva em $\mathcal{F}$.

Demonstração. Veja [Rud70] Teorema 6.2, página 118.

Definição 2.1.25. Sejam $\mu$ uma medida em $\mathcal{F}$ e $\nu$ uma medida complexa (medida real) em $\mathcal{F}$. Dizemos que $\nu$ é absolutamente contínua com respeito a $\mu$ se $\nu(E)=0$ para todo $E \in \mathcal{F}$ satisfazendo $\mu(E)=0$. Neste caso, denotamos $\nu \prec \mu$.

Exemplo 2.1.26. Seja $f: \Omega \rightarrow \mathbb{C}$ uma função integrável. Definimos $\nu: \mathcal{F} \rightarrow \mathbb{C}$ por

$$
\nu(E)=\int_{E} f d \mu
$$

para cada $E \in \mathcal{F}$. Então $\nu$ é uma medida complexa absolutamente contínua com respeito a $\mu$.

Teorema 2.1.27. Sejam $\mu$ uma medida em $\mathcal{F}$ e $\nu$ uma medida complexa em $\mathcal{F}$ absolutamente contínua com respeito a $\mu$. Então para todo $\epsilon>0$ existe $\delta>0$ tal que $|\nu(E)|<\epsilon$ sempre que $E \in \mathcal{F}$ e $|\mu(E)|<\delta$.

Demonstração. Veja [Rud70] Teorema 6.11, página 125. 


\subsection{Probabilidade}

Nesta seção iremos introduzir conceitos da teoria de probabilidade que usaremos no capítulo 3. Indicamos como referência principal o livro [Dur13].

Definição 2.2.1. Um espaço de probabilidade é um espaço de medida $(\Omega, \mathcal{F}, \mathbb{P})$ tal que $\mathbb{P}(\Omega)=1$.

Ao longo desta seção vamos fixar um espaço de probabilidade $(\Omega, \mathcal{F}, \mathbb{P})$.

Definição 2.2.2. Um vetor aleatório real em $(\Omega, \mathcal{F}, \mathbb{P})$ é uma função $\xi: \Omega \rightarrow \mathbb{R}^{n}$ mensurável. Caso $n=1$, dizemos que $\xi$ é uma variável aleatória.

Definição 2.2.3. Sejam $\xi_{1}, \ldots, \xi_{n}$ variáveis aleatórias. Definimos o vetor aleatório $\left(\xi_{1}, \ldots, \xi_{n}\right)$ : $\Omega \rightarrow \mathbb{R}^{n}$ por $\left(\xi_{1}, \ldots, \xi_{n}\right)(\omega)=\left(\xi_{1}(\omega), \ldots, \xi_{n}(\omega)\right)$ para cada $\omega \in \Omega$.

\section{Definição 2.2.4.}

1. Sejam $\xi: \Omega \rightarrow \mathbb{R}^{n}$ um vetor aleatório e $A \in \operatorname{Bor}\left(\mathbb{R}^{n}\right)$. Definimos o conjunto $\{\xi \in A\}$ $\operatorname{por} \xi^{-1}[A]$;

2. Sejam $\xi_{1}, \ldots, \xi_{n}$ variáveis aleatórias e $A_{1}, \ldots, A_{n} \in \operatorname{Bor}(\mathbb{R})$. Definimos $\left\{\xi_{1} \in A_{1}, \ldots, \xi_{n} \in\right.$ $\left.A_{n}\right\}$ como sendo $\left\{\left(\xi_{1}, \ldots, \xi_{n}\right) \in A_{1} \times \ldots \times A_{n}\right\}$;

3. Sejam $\xi$ uma variável aleatória e $t \in \mathbb{R}$. Definimos o conjunto $\{\xi \leq t\}$ como sendo $\xi^{-1}[(-\infty, t]]$. Analogamente, definimos os conjuntos $\{\xi<t\}$ e $\{\xi=t\}$ como sendo $\xi^{-1}[(-\infty, t)]$ e $\xi^{-1}[\{t\}]$, respectivamente.

Observação 2.2.5. Note também que dadas variáveis aleatórias $\xi_{1}, \ldots, \xi_{n}$ e $A_{1}, \ldots, A_{n} \in$ $\operatorname{Bor}(\mathbb{R})$, temos que $\left\{\left(\xi_{1}, \ldots, \xi_{n}\right) \in A_{1} \times \ldots \times A_{n}\right\}=\bigcap_{i=1}^{n}\left\{\xi_{i} \in A_{i}\right\}$.

Definição 2.2.6. Seja $\xi: \Omega \rightarrow \mathbb{R}$ uma variável aleatória. A distribuição de $\xi$ é a medida $\mu$ em $(\Omega, \mathcal{F})$ dada por $\mu(A)=\mathbb{P}\left(\xi^{-1}[A]\right)$ para cada $A \in$ Bor $(\mathbb{R})$. A função distribuição de $\xi e ́$ a função $F: \mathbb{R} \rightarrow[0,1]$ dada por $F(t)=\mathbb{P}(\xi \leq t)$.

Definição 2.2.7. Seja $\xi: \Omega \rightarrow \mathbb{R}$ uma variável aleatória. Dizemos que $\xi$ tem distribuição uniforme se sua medida distribuição $\mu$ é dada por $\mu(A)=m_{\mathbb{R}}(A \cap(0,1))$ para cada $A \in$ $\operatorname{Bor}(\mathbb{R})$.

Proposição 2.2.8. Seja $F: \mathbb{R} \rightarrow[0,1]$ a função distribuição de uma variável aleatória $\xi: \Omega \rightarrow \mathbb{R}$. Então:

1. F é crescente;

2. $\lim _{x \rightarrow \infty} F(x)=1 e \lim _{x \rightarrow-\infty} F(x)=0$;

3. $\lim _{y \rightarrow x^{+}} F(y)=F(x)$; 
4. $\lim _{y \rightarrow x^{-}} F(y)=P(\xi<x)$;

5. $P(\xi=x)=F(x)-\lim _{y \rightarrow x^{-}} F(y)$.

Reciprocamente, se $F: \mathbb{R} \rightarrow \mathbb{R}$ satisfaz as propriedades 1,2 e 3 , então existe uma variável aleatória $\xi$ em $((0,1)$, Bor $(0,1))$ tal que $F$ é a função distribuição de $\xi$.

Demonstração. As propriedades $1-5$ seguem da definição de função distribuição e das propriedades da medida de probabilidade $\mathbb{P}$.

Reciprocamente, seja $F: \mathbb{R} \rightarrow \mathbb{R}$ uma função satisfazendo 1,2 e 3 . Seja $(\Omega, \mathcal{F}, \mathbb{P})$ o espaço mensurável $((0,1), \operatorname{Bor}(0,1))$ equipado com a medida de Lebesgue. Definimos $\xi:(0,1) \rightarrow \mathbb{R}$ por

$$
\xi(t)=\sup \{y \in(0,1): F(y)<t\} \text { para cada } t \in(0,1) .
$$

Veja que $\xi$ está bem definida pois o supremo está sendo tomado em um conjunto limitado. Vamos mostrar que $\{t \in(0,1): \xi(t) \leq x\}=(0, F(x)$ ]. Veja que isto é o suficiente para concluir a demonstração. De fato, teremos que $F$ é a função distribuição de $\xi$ pois $\mathbb{P}(\xi \leq$ $x)=\mathbb{P}(0, F(x)]=F(x)$ e além disso, $\xi^{-1}[(-\infty, x]]=(0, F(x)]$ é um boreliano e então $\xi$ é mensurável pela Proposição 2.1.6.

Veja que $t \leq F(x)$ implica $\xi(t) \leq x$. De fato, temos que $x \notin\{y \in(0,1): F(y)<t\}$ pois caso contrário $F(x)<t$. Caso $x<\xi(t)$, existiria $y \in(0,1)$ tal que $x<y$ e $F(y)<t$. Como $F$ é crescente isto implicaria que $F(x) \leq F(y)<t$, uma contradição. Logo $x \geq \xi(t)$ e $\operatorname{assim}(0, F(x)] \subset \xi^{-1}[(0, x]]$. Por outro lado, se $t>F(x)$, existe por 3) um $\epsilon>0$ tal que $F(x+\epsilon)<t$. Mas então $x<x+\epsilon \leq \xi(t)$, ou seja, $\xi^{-1}(0, x] \subset(0, F(x)]$.

Definição 2.2.9. O valor esperado de uma variável aleatória $\xi: \Omega \rightarrow \mathbb{R}$ é o valor $\mathbb{E}[\xi]$ definido por

$$
\mathbb{E}[\xi]=\int_{\Omega} \xi(\omega) d \mathbb{P}(\omega) .
$$

A variância é o valor var $(\xi)$ definido por

$$
\operatorname{var}(\xi)=\mathbb{E}\left[\xi^{2}\right]-\mathbb{E}[\xi]^{2}
$$

Vamos definir agora o conceito de independência. Estamos interessados principalmente no conceito de variáveis aleatórias independentes, que será utilizado ao decorrer do texto. Entretanto, alguns outros conceitos de independência também serão tratados pois irão simplificar alguns resultados.

\section{Definição 2.2.10.}

1. Sejam $\xi_{1}, \ldots, \xi_{n}$ variáveis aleatórias no espaço de probabilidade $(\Omega, \mathcal{F}, \mathbb{P})$. Dizemos que estas variáveis aleatórias são independentes se para quaisquer borelianos $B_{1}, \ldots, B_{n} \in$ $\operatorname{Bor}(\mathbb{R})$, temos $\mathbb{P}\left(\bigcap_{i=1}^{n}\left\{\xi_{i} \in B_{i}\right\}\right)=\prod_{i=1}^{n} \mathbb{P}\left(\xi_{i} \in B_{i}\right)$. 
2. Dizemos que $A_{1}, \ldots, A_{n} \in \mathcal{F}$ são independentes se para qualquer $I \subset\{1, \ldots, n\}$, temos $\mathbb{P}\left(\bigcap_{i \in I} A_{i}\right)=\prod_{i \in I} \mathbb{P}\left(A_{i}\right)$.

3. Dizemos que coleções $\mathcal{A}_{1}, \ldots, \mathcal{A}_{n} \subset \mathcal{P}(\Omega)$ contendo $\Omega$ são independentes se para qualquer $A_{i} \in \mathcal{A}_{i}$ para $i=1, \ldots, n$, temos $\mathbb{P}\left(\bigcap_{i=1}^{n} A_{i}\right)=\prod_{i=1}^{n} \mathbb{P}\left(A_{i}\right)$.

No caso em que precisamos dizer quando uma sequência de variáveis aleatórias é independente, precisamos de outra definição.

Definição 2.2.11. Seja $\left(\xi_{n}\right)_{n \in \mathbb{N}}$ uma sequência de variáveis aleatórias em $(\Omega, \mathcal{F}, \mathbb{P})$. Dizemos que esta sequência é independente se para todo subconjunto finito $F \subset \mathbb{N}$, temos que $\left\{\xi_{i}\right.$ : $i \in F\}$ é um conjunto de variáveis aleatórias independentes.

Lema 2.2.12. Sejam $\mathcal{A}_{1}, \ldots, \mathcal{A}_{n} \subset \mathcal{P}(\Omega)$ independentes tais que cada $\mathcal{A}_{i}$ contém $\Omega$ e é um $\pi$-sistema. Então $\sigma\left(\mathcal{A}_{1}\right), \ldots, \sigma\left(\mathcal{A}_{n}\right)$ são independentes.

Demonstração. Vamos mostrar que $\sigma\left(\mathcal{A}_{1}\right), \mathcal{A}_{2}, \ldots, \mathcal{A}_{n}$ são independentes, e o resultado seguirá por indução. Seja $A_{i} \in \mathcal{A}_{i}$ para cada $i=2, \ldots, n$. Definimos $A=\bigcap_{i=2}^{n} A_{i} \mathrm{e}$

$$
\mathcal{C}=\{B \subset \Omega: \mathbb{P}(B \cap A)=\mathbb{P}(B) \mathbb{P}(A)\}
$$

Vamos mostrar que $\mathcal{C}$ é um $\lambda$-sistema. De fato, $\Omega \in \mathcal{C}$ e $B_{n} \nearrow B$ com $B_{n} \in \mathcal{C}$ implicam pela Proposição 2.1.2 que

$$
\mathbb{P}(B \cap A)=\lim _{n \in \mathbb{N}} \mathbb{P}\left(B_{n} \cap A\right)=\lim _{n \in \mathbb{N}} \mathbb{P}\left(B_{n}\right) P(A)=\mathbb{P}(B) \mathbb{P}(A) .
$$

Por fim, sejam $B, C \in \mathcal{C}$ com $B \subset C$. Então

$\mathbb{P}((C \backslash B) \cap A)=\mathbb{P}((C \cap A) \backslash(B \cap A))=\mathbb{P}(C \cap A)-\mathbb{P}(B \cap A)=\mathbb{P}(A)(\mathbb{P}(C)-\mathbb{P}(B))=\mathbb{P}(C \backslash B) \mathbb{P}(A)$.

Como $\mathcal{A}_{1} \subset \mathcal{C}$, segue do Teorema 2.1.5 que $\sigma\left(\mathcal{A}_{1}\right) \subset \mathcal{C}$ e para todo $C \in \sigma\left(\mathcal{A}_{1}\right)$, temos

$$
\mathbb{P}\left(C \cap \bigcap_{i=2}^{n} A_{i}\right)=\mathbb{P}(C) . \prod_{i=2}^{n} \mathbb{P}\left(A_{i}\right)
$$

Portanto, segue também que a igualdade acima vale para cada $C \in \sigma\left(\mathcal{A}_{1}\right)$ e para todo $A_{i} \in$ $\mathcal{A}_{i}$, com $i=2, \ldots, n$. Isto mostra que $\sigma\left(\mathcal{A}_{1}\right), \mathcal{A}_{2}, \ldots, \mathcal{A}_{n}$ são independentes, como queríamos mostrar.

O lema a seguir nos dá uma condição suficiente para que variáveis aleatórias sejam independentes.

Lema 2.2.13. Sejam $\xi_{1}, \ldots, \xi_{n}$ variáveis aleatórias em $(\Omega, \mathcal{F}, \mathbb{P})$. Suponha que para todos $x_{1}, \ldots, x_{n} \in(-\infty, \infty]$, temos $\mathbb{P}\left(\xi_{1} \leq x_{1}, \ldots, \xi_{n} \leq x_{n}\right)=\prod_{i=1}^{n} \mathbb{P}\left(\xi_{i} \leq x_{i}\right)$. Então $\xi_{1}, \ldots, \xi_{n}$ s $\tilde{a} o$ independentes. 
Demonstração. Definimos $\mathcal{A}_{i}=\left\{\left\{\xi_{i} \leq y\right\}: y \in(-\infty, \infty]\right\}$. Temos que $\mathcal{A}_{i}$ forma um $\pi$-sistema pois $\left\{\xi_{i} \leq y\right\} \cap\left\{\xi_{i} \leq z\right\}=\left\{\xi_{i} \leq \min (y, z)\right\}$. Além disso, $\mathcal{A}_{1}, \ldots, \mathcal{A}_{n}$ são independentes por hipótese. Pelo Lema 2.2.12, temos que $\sigma\left(\mathcal{A}_{1}\right), \ldots, \sigma\left(\mathcal{A}_{n}\right)$ são independentes. Se $\sigma\left(\xi_{i}\right)=\left\{\xi_{i}^{-1}[A]: A \in \operatorname{Bor}(\mathbb{R})\right\}$, então $\sigma\left(\mathcal{A}_{i}\right) \subset \sigma\left(\xi_{i}\right)$ pois $\sigma\left(\xi_{i}\right)$ é uma $\sigma$-álgebra contendo os borelianos $\left\{\xi_{i} \leq y\right\}$ para cada $y \in \mathbb{R}$. Vejamos que $\sigma\left(\xi_{i}\right) \subset \sigma\left(\mathcal{A}_{i}\right)$. De fato, como $\xi_{i}^{-1}(-\infty, y] \in \sigma\left(\mathcal{A}_{i}\right)$ para cada $y \in \mathbb{R}$, temos que $\xi_{i}$ é $\sigma\left(\mathcal{A}_{i}\right)$-mensurável pela Proposição 2.1.6, isto é, $\xi_{i}^{-1}[A] \in \sigma\left(\mathcal{A}_{i}\right)$ para todo $A \in \operatorname{Bor}(\mathbb{R})$ e assim $\sigma\left(\xi_{i}\right) \subset \sigma\left(\mathcal{A}_{i}\right)$.

Logo $\sigma\left(\xi_{1}\right), \ldots, \sigma\left(\xi_{n}\right)$ são independentes e portanto $\xi_{1}, \ldots, \xi_{n}$ são independentes.

Proposição 2.2.14. Sejam $\xi_{1}, \ldots, \xi_{n}$ variáveis aleatórias independentes em $(\Omega, \mathcal{F}, \mathbb{P})$ com distribuição $\mu_{1}, \ldots, \mu_{n}$, respectivamente. Então $\left(\xi_{1}, \ldots, \xi_{n}\right)$ tem distribuição $\mu_{1} \otimes \ldots \otimes \mu_{n}$.

Demonstração. Sejam $A_{1}, \ldots, A_{n}$ borelianos em $\mathbb{R}$. Então pela independência das variáveis aleatórias temos

$$
\begin{aligned}
\mathbb{P}\left(\left(\xi_{1}, \ldots, \xi_{n}\right) \in A_{1} \times \ldots \times A_{n}\right)=\mathbb{P}\left(\xi_{1} \in A_{1}, \ldots, \xi_{n} \in A_{n}\right) & =\prod_{i=1}^{n} \mathbb{P}\left(\xi_{i} \in A_{i}\right) \\
& =\prod_{i=1}^{n} \mu_{i}\left(A_{i}\right) \\
& =\mu_{1} \otimes \ldots \otimes \mu_{n}\left(A_{1} \times \ldots \times A_{n}\right) .
\end{aligned}
$$

Pela unicidade da medida produto $\mu_{1} \otimes \ldots \otimes \mu_{n}$, segue que

$$
\mathbb{P}\left(\left(\xi_{1}, \ldots, \xi_{n}\right) \in B\right)=\mu_{1} \otimes \ldots \otimes \mu_{n}(B)
$$

para todo $B \in \mathcal{F}^{n}$ e o resultado segue.

Nosso objetivo agora é construir variáveis aleatórias independentes com distribuições previamente dadas. Para o caso no qual o número de distribuições é finito, a construção é dada pelo resultado seguinte.

Teorema 2.2.15. Sejam $F_{1}, \ldots, F_{n}: \mathbb{R} \rightarrow[0,1]$ funções de distribuição. Então existem variáveis aleatórias independentes $\xi_{1}, \ldots, \xi_{n}$ definidas em um espaço de probabilidade $(\Omega, \mathcal{F}, \mathbb{P})$ tais que $\mathbb{P}\left(\xi_{i} \leq t\right)=F_{i}(t)$ para todo $t \in \mathbb{R}$.

Demonstração. Seja $Y_{i}:(0,1) \rightarrow \mathbb{R}$ uma variável aleatória cuja função distribuição é $F_{i}$. Seja $\mu_{i}$ a distribuição de $Y_{i}$. Sejam $\Omega=(0,1)^{n}$ e $\xi_{i}:(0,1)^{n} \rightarrow \mathbb{R}$ como sendo a $i$-ésima projeção e $\mathbb{P}=\mu_{1} \otimes \ldots \otimes \mu_{n}$. Então $\mathbb{P}$ é uma medida de probabilidade e para todo $i=1, \ldots, n$, temos que $\mathbb{P}\left(\xi_{i} \leq x\right)=\mu_{i}(-\infty, x]=F_{i}(x)$. Além disso, para cada $x_{1}, \ldots, x_{n} \in(-\infty, \infty]$, temos

$$
\mathbb{P}\left(\xi_{1} \leq x_{1}, \ldots, \xi_{n} \leq x_{n}\right)=\mathbb{P}\left(\left(-\infty, x_{1}\right] \times \ldots \times\left(-\infty, x_{n}\right]\right)=\prod_{i=1}^{n} \mathbb{P}\left(\xi_{i} \leq x_{i}\right)
$$

Pelo Lema 2.2.13, $\xi_{1}, \ldots, \xi_{n}$ são independentes. 
Agora vamos tratar o caso no qual é dada uma sequência infinita de distribuições. Por mais que a ideia seja a mesma, precisamos de um pouco mais de teoria para construir a medida. Mais precisamente, precisamos do teorema da extensão de Kolmogorov.

Definição 2.2.16. Definimos a $\sigma$-álgebra produto $\sigma\left(\mathbb{R}^{\mathbb{N}}\right)$ em $\mathbb{R}^{\mathbb{N}}$ como sendo

$$
\sigma\left(\left\{\prod_{i=1}^{n} B_{i} \times \prod_{i>n} \mathbb{R}: n \in \mathbb{N} \text { e } B_{1}, \ldots, B_{n} \in \operatorname{Bor}(\mathbb{R})\right\}\right)
$$

Definição 2.2.17. Para cada $n \in \mathbb{N}$, seja $\mu_{n}: \mathbb{R}^{n} \rightarrow[0,1]$ uma medida de probabilidade. A sequência $\left(\mu_{n}\right)_{n \in \mathbb{N}}$ é dita consistente se

$$
\mu_{n+1}\left(\prod_{i=1}^{n}\left(a_{i}, b_{i}\right] \times \mathbb{R}\right)=\mu_{n}\left(\prod_{i=1}^{n}\left(a_{i}, b_{i}\right]\right)
$$

para todos $a_{i}, b_{i} \in \mathbb{R}$ e $n \in \mathbb{N}$.

Teorema 2.2.18 (Teorema da extensão de Kolmogorov). Sejam $\left(\mu_{n}\right)_{n \in \mathbb{N}}$ uma sequência de medidas de probabilidade consistente. Então existe uma única medida de probabilidade $\mathbb{P}$ em $\left(\mathbb{R}^{\mathbb{N}}, \sigma\left(\mathbb{R}^{\mathbb{N}}\right)\right)$ satisfazendo

$$
\mathbb{P}\left(\prod_{i=1}^{n}\left(a_{i}, b_{i}\right] \times \prod_{i>n} \mathbb{R}\right)=\mu_{n}\left(\prod_{i=1}^{n}\left(a_{i}, b_{i}\right]\right)
$$

para todos $a_{i}, b_{i} \in \mathbb{R}$ e $n \in \mathbb{N}$.

Demonstração. Indicamos [Dur13] Teorema A.3.1.

Teorema 2.2.19. Seja $\left(F_{n}\right)_{n \in \mathbb{N}}$ uma sequência de funções distribuição. Então existe uma sequência de variáveis aleatórias $\left(\xi_{n}\right)_{n \in \mathbb{N}}$ independentes em um espaço de probabildiade $(\Omega, \mathcal{F}, \mathbb{P})$ tais que $\mathbb{P}\left(\xi_{n} \leq t\right)=F_{n}(t)$ para todo $t \in \mathbb{R}$ e todo $n \in \mathbb{N}$.

Demonstração. Seja $(\Omega, \mathcal{F})=\left(\mathbb{R}^{\mathbb{N}}, \sigma\left(\mathbb{R}^{\mathbb{N}}\right)\right)$. Para cada $i \in \mathbb{N}$, fixamos uma variável aleatória $Y_{i}$ cuja função distribuição é $F_{i}$ e definimos $\mu_{i}$ como sendo a medida distribuição de $Y_{i}$. Definimos então $\mathbb{P}_{n}=\mu_{1} \otimes \ldots \otimes \mu_{n}$. Veja que

$$
\begin{aligned}
\mathbb{P}_{n+1}\left(\left(a_{1}, b_{1}\right] \times \ldots \times\left(a_{n}, b_{n}\right] \times \mathbb{R}\right) & =\mu_{1} \otimes \ldots \otimes \mu_{n+1}\left(\left(a_{1}, b_{1}\right] \times \ldots \times\left(a_{n}, b_{n}\right] \times \mathbb{R}\right) \\
& =\mu_{1} \otimes \ldots \otimes \mu_{n}\left(\left(a_{1}, b_{1}\right] \times \ldots \times\left(a_{n}, b_{n}\right]\right) \\
& =\mathbb{P}_{n}\left(\left(a_{1}, b_{1}\right] \times \ldots \times\left(a_{n}, b_{n}\right]\right) .
\end{aligned}
$$

Então $\left(\mathbb{P}_{n}\right)_{n \in \mathbb{N}}$ é uma sequência consistente e pelo teorema da extensão de Kolmogorov existe uma única medida de probabilidade $\mathbb{P}$ com as propriedades dadas pelo enunciado. Seja $\xi_{n}: \mathbb{R}^{\mathbb{N}} \rightarrow \mathbb{R}$ a $n$-ésima projeção, então

$$
\left.\mathbb{P}\left(\xi_{i} \leq t\right)=\mathbb{P}\left(\prod_{j=1}^{i-1} \mathbb{R} \times(-\infty, t] \times \prod_{j>i+1} \mathbb{R}\right)=\mathbb{P}_{i}\left(\prod_{j=1}^{i-1} \mathbb{R} \times(-\infty, t]\right)=\mu_{i}(-\infty, t]\right)=F_{i}(t) .
$$


Além disso, se $F \subset \mathbb{N}$ é finito e $n_{0}=\max F$, temos

$$
\mathbb{P}\left(\xi_{i} \leq x_{i}: i \in F\right)=\mathbb{P}_{n_{0}}\left(\xi_{i} \leq x_{i}: i \in F\right)=\prod_{i \in F} \mathbb{P}_{n_{0}}\left(\xi_{i} \leq x_{i}\right)=\prod_{i \in F} \mathbb{P}\left(\xi_{i} \leq x_{i}\right)
$$

Isto mostra que $\left(\xi_{n}\right)_{n \in \mathbb{N}}$ é uma sequência de variáveis aleatórias independentes.

Corolário 2.2.20. Seja $\left(\mu_{n}\right)_{n \in \mathbb{N}}$ uma medidas de probabilidade. Então existe uma sequência de variáveis aleatórias $\left(\xi_{n}\right)_{n \in \mathbb{N}}$ independentes em um espaço de probabildiade $(\Omega, \mathcal{F}, \mathbb{P})$ tais que $\mathbb{P}\left(\xi_{n}^{-1}[A]\right)=\mu_{n}(A)$ para cada $A \in \operatorname{Bor}(\mathbb{R})$ e para todo $n \in \mathbb{N}$.

Demonstração. Para cada $n \in \mathbb{N}$, definimos uma função distribuição $F_{n}(t)=\mu_{n}((-\infty, t])$. Pelo teorema acima, existe uma sequência de variáveis aleatórias $\left(\xi_{n}\right)_{n \in \mathbb{N}}$ independentes em um espaço de probabilidade $(\Omega, \mathcal{F}, \mathbb{P})$ tais que $\mathbb{P}\left(\xi_{n} \leq t\right)=F_{n}(t)$ para todo $t \in \mathbb{R}$ e $n \in \mathbb{N}$. Fixado $n \in \mathbb{N}$, sejam $\mathcal{A}=\{(-\infty, t]: t \in \mathbb{R}\}$ e $\mathcal{B}=\left\{A \in \operatorname{Bor}(\mathbb{R}): \mathbb{P}\left(\xi_{n}^{-1}\right)[A]=\mu_{n}(A)\right\}$. Note que $\mathcal{A}$ é um $\pi$-sistema e $\mathcal{B}$ é um $\lambda$-sistema. Segue então do Lema 2.1.5 que $\operatorname{Bor}(\mathbb{R})=$ $\sigma(\mathcal{A})=\mathcal{B}$, isto é, $\mathbb{P}\left(\xi_{n}^{-1}[A]\right)=\mu_{n}(A)$ para todo $A \in \operatorname{Bor}(\mathbb{R})$.

Vamos terminar a seção enunciando um resultado clássico da teoria de probabilidade que usaremos na seção 3.

Definição 2.2.21. Sejam $\left(\xi_{n}\right)_{n \in \mathbb{N}}$ uma sequência de variáveis aleatórias em $(\Omega, \mathcal{F}, \mathbb{P})$ e $\left(F_{n}\right)_{n \in \mathbb{N}}$ a sequência associada das funções distribuição. Seja $\xi$ uma variável aleatória em $(\Omega, \mathcal{F}, \mathbb{P})$ e $F$ sua função distribuição. Dizemos que a sequência $\left(\xi_{n}\right)_{n \in \mathbb{N}}$ converge fracamente a $\xi$ se $F_{n}(t) \rightarrow F(t)$ para todo ponto $t$ no qual $F$ é contínua.

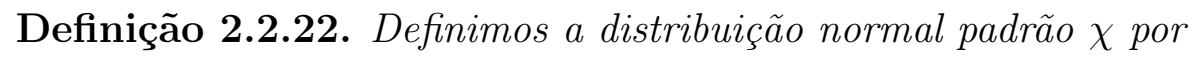

$$
\chi(x)=\int_{-\infty}^{x}(2 \pi)^{-1 / 2} e^{-x^{2} / 2} d x \text { para todo } x \in \mathbb{R} .
$$

Teorema 2.2.23 (Teorema da Convergência Central). Seja $\left(\xi_{n}\right)_{n \in \mathbb{N}}$ uma sequência de variáveis aleatórias independentes em $(\Omega, \mathcal{F}, \mathbb{P})$ e igualmente distribuídas. Sejam $\mu=\mathbb{E}\left[\xi_{1}\right]$, $\sigma^{2}=\operatorname{var}\left(\xi_{1}\right)$ e $S_{n}=\xi_{1}+\ldots+\xi_{n}$. Então

$$
\left(S_{n}-n \mu\right) /\left(\sigma n^{1 / 2}\right) \rightarrow \chi
$$

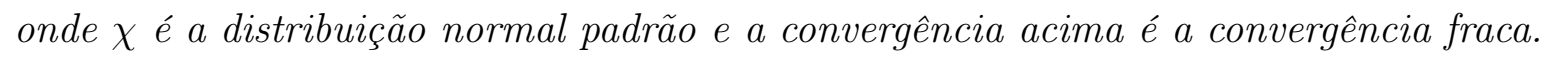

Demonstração. Indicamos [Dur13] Teorema 3.4.1. 


\subsection{Análise Funcional}

Nesta seção iremos relembrar alguns resultados clássicos de Análise Funcional e introduzir alguns conceitos que serão utilizados ao decorrer do texto. Indicamos como principal referência o [Meg98]. Iremos denotar por $\mathbb{F}$ o corpo dos escalares $\mathbb{R}$ ou $\mathbb{C}$.

\subsubsection{Conceitos e teoremas básicos}

Definição 2.3.1. Seja $X$ um espaço normado. A bola unitária é o conjunto $B_{X}=\{x \in X$ : $\|x\| \leq 1\}$. A esfera unitária é o conjunto $S_{X}=\{x \in X:\|x\|=1\}$. A bola aberta centrada em $x_{0} \in X$ e com raio $r>0$ é o conjunto $B\left(x_{0} ; r\right)=\left\{x \in X:\left\|x-x_{0}\right\|<r\right\}$.

Definição 2.3.2. Sejam $X$ um espaço normado, $A \subset X$ um subconjunto não vazio e $x \in X$. Definimos

$$
d(x, A)=\inf _{a \in A}\|x-a\| .
$$

Lema 2.3.3 (Riesz). Sejam $X$ um espaço normado, $M$ um subespaço próprio e fechado de $X$ e $0<\theta<1$. Então existe $x \in S_{X}$ tal que $d(x, M) \geq \theta$.

Demonstração. Ver [Meg98] Lema 3.4.18, página 325.

Observação 2.3.4. Seja $\tau>0$ e $0<\theta^{\prime}<\tau$. Sob as condições acima, podemos aplicar o resultado para $\theta=\theta^{\prime} / \tau$ e obter que existe $x \operatorname{com}\|x\|=\tau$ e $d(x, M) \geq \theta^{\prime}$.

Teorema 2.3.5 (Hahn-Banach). Sejam X um espaço normado, $Y$ um subespaço de $X$ e $f_{0}$ um funcional linear limitado em $Y$. Então existe um funcional linear limitado $f$ em $X$ tal que $\|f\|=\left\|f_{0}\right\|$ e $f(y)=f_{0}(y)$ para todo $y \in Y$.

Demonstração. Ver [Meg98] Teorema 1.9.6, página 75.

Corolário 2.3.6. Sejam $X$ um espaço normado e $x \in X \backslash\{0\}$. Então existe um funcional f em $X$ tal que $\|f\|=1$ e $f(x)=\|x\|$.

Demonstração. Ver [Meg98] Corolário 1.9.8, página 76.

Corolário 2.3.7. Seja $X$ um espaço normado separável. Então existe uma sequência $\left(x_{n}^{*}\right)_{n \in \mathbb{N}}$ em $X^{*}$ tal que

$$
\|x\|=\sup _{n}\left|x_{n}^{*}(x)\right| \text { para todo } x \in X .
$$

Demonstração. Seja $\left(x_{n}\right)_{n \in \mathbb{N}}$ uma sequência densa em $X$ e contida em $X \backslash\{0\}$. Pelo Corolário 2.3.6, existe $x_{n}^{*}$ tal que $\left\|x_{n}^{*}\right\|=1$ e $x_{n}^{*}\left(x_{n}\right)=\left\|x_{n}\right\|$ para cada $n \in \mathbb{N}$. Sejam $x \in X$ e $\epsilon>0$. Tome $N \in \mathbb{N}$ tal que $\left\|x-x_{N}\right\|<\epsilon$. Então

$$
|\|x\|-| x_{N}^{*}(x)\left|\left\|\leq\left|\|x\|-\left\|x_{N}\right\|\right|+\left|\left\|x_{N}\right\|-\right| x_{N}^{*}(x)\right\| \leq\right|\|x\|-\left\|x_{N}\right\||+| x_{N}^{*}\left(x-x_{N}\right) \mid<2 \epsilon .
$$


segue então que $\|x\| \leq \sup _{n}\left|x_{n}^{*}(x)\right|+2 \epsilon$. Como $\epsilon$ foi escolhido arbitrariamente, segue que $\|x\| \leq \sup _{n}\left|x_{n}^{*}(x)\right|$. Por outro lado, para cada $n \in \mathbb{N}$, temos $\left|x_{n}^{*}(x)\right| \leq\left\|x_{n}^{*}\right\|\|x\|=\|x\|$ e o resultado segue.

Definição 2.3.8. Sejam $X$ espaço normado e $Y$ um subespaço de $X$. Definimos o espaço quociente $X / Y$ como sendo a coleção $\{\bar{x}: x \in X\}$, onde $\bar{x}$ é o conjunto $\{y \in X: x-y \in Y\}$ para cada $x \in X$.

Teorema 2.3.9. Sejam $X$ espaço normado e $Y$ um subespaço fechado de $X$. Então $X / Y$ é um espaço normado com a norma

$$
\|\bar{x}\|=\inf _{y \in \bar{x}}\|y\| \text { para cada } \bar{x} \in X / Y
$$

Além disso, se X é um espaço de Banach, então X/Y também é um espaço de Banach.

Demonstração. Veja [Meg98] Teorema 1.7.4. e Teorema 1.7.7.

Definição 2.3.10. Sejam $X$ e $Y$ espaços normados. Definimos $\mathcal{L}(X, Y)$ como sendo o espaço de todos operadores lineares e contínuos $T: X \rightarrow Y$.

Definição 2.3.11. Sejam $X$ espaço normado e $Y$ um subespaço de $X$. Definimos a função quociente como sendo a função $\pi: X \rightarrow X / Y$ dada por $\pi(x)=\bar{x}$.

Lembramos que uma aplicação linear $T: X \rightarrow Y$ entre espaços normados $X$ e $Y$ é aberta se para todo aberto $V$ em $X$, temos que $T(V)$ é um aberto em $Y$.

Proposição 2.3.12. Sejam $X$ espaço normado e $Y$ um subespaço de $X$. Então a função quociente $\pi$ é uma transforamção linear contínua e aberta. Além disso, se $Y \neq X$, então temos $\|\pi\|=1$.

Em geral, podemos definir uma função quociente entre dois espaços normados.

Definição 2.3.13. Sejam $X$ e $Z$ espaços de Banach. Dizemos que um operador $q \in \mathcal{L}(X, Z)$ é um operador quociente se q é sobrejetora e aberta.

Teorema 2.3.14. Sejam $X$ e $Z$ espaços de Banach. Então $Z$ é isomorfo a um quociente de $X$ se e somente se existe uma função quociente $q: X \rightarrow Z$

Demonstração. Suponha que $Z$ é isomorfo a um quociente de $X$. Então existem $Y$ subespaço fechado de $X$ e $T: X / Y \rightarrow Z$ isomorfismo. Então se $\pi: X \rightarrow X / Y$ é a função quociente, o operador $q=T \circ \pi$ é um operador quociente entre $X$ e $Z$.

Por outro lado, se existe um operador quociente $q: X \rightarrow Z$, então o resultado segue de [Meg98] Teorema 1.7.13.

Um conceito que usaremos bastante é o de subespaço complementado. 
Definição 2.3.15. Sejam $X$ um espaço vetorial e $N$ e $M$ subespaços de $X$. Dizemos que $X$ é a soma interna de $N$ e $M$ se $N \cap M=\{0\}$ e se para todo $x \in X$ existem $n \in N$ e $m \in M$ tais que $x=n+m$.

Definição 2.3.16. Sejam $X$ um espaço normado e $M$ um subespaço fechado de $X$. Dizemos que $M$ é um subespaço complementado de $X$ se existe um subespaço $N$ de $X$ também fechado tal que $X$ é a soma direta de $M$ e $N$.

Uma forma mais direta de verificar se um subespaço é complementado é através de projeções contínuas.

Definição 2.3.17. Seja $X$ um espaço normado. Uma projeção contínua é uma função $P$ : $X \rightarrow X$ linear e contínua tal que $P(P(x))=P(x)$ para todo $x \in X$.

Proposição 2.3.18. Sejam $X$ um espaço de Banach e $M$ um subespaço fechado de $X$. Então $M$ é um subespaço complementado de $X$ se e somente se existe uma projeção continua $P: X \rightarrow X$ cuja imagem é $M$.

Demonstração. Ver [Meg98] Corolário 3.2.15, página 299.

Vamos denotar o espaço dual de um espaço normado $X$ por $X^{*}$, isto é, $X^{*}=\left\{x^{*}: X \rightarrow\right.$ $\mathbb{F}: x^{*}$ é linear e contínua $\}$. Denotamos o espaço bidual, isto é, o espaço $\left(X^{*}\right)^{*}$, simplesmente por $X^{* *}$.

Definição 2.3.19. Definimos a inclusão canônica $Q: X \rightarrow X^{* *}$ por $Q(x)\left(x^{*}\right)=x^{*}(x)$ para cada $x \in X$ e $x^{*} \in X^{*}$.

Dizemos que um espaço normado é reflexivo se $Q$ é um operador sobrejetor.

Agora vamos relembrar os conceitos de topologia fraca e fraca* em espaços de Banach. Antes disso, vamos definir o que é um espaço vetorial topológico.

Definição 2.3.20. Seja $X$ um espaço vetorial e $\tau$ uma topologia em $X$ com as seguintes propriedades:

1. A função soma $S: X \times X \rightarrow X$ dada por $S(x, y)=x+y$ é contínua.

2. A função multiplicação por escalar $M: \mathbb{F} \times X$ dada por $M(\alpha, x)=\alpha x$ é contínua.

Então dizemos que o par $(X, \tau)$ é um espaço vetorial topológico.

É claro que o exemplo mais básico de espaço vetorial topológico são os próprios espaços normados. Além disso, estes são espaços localmente convexos.

Definição 2.3.21. Seja $X$ um espaço vetorial topológico. Dizemos que $X$ é um espaço localmente convexo se existe uma base de abertos convexos para a topologia de $X$. 
Definição 2.3.22. Sejam $X$ um espaço vetorial topológico e $A$ um subconjunto de $X$. Dizemos que $A$ é limitado se para toda vizinhança $U$ de 0 , existe um $s_{U}>0$ tal que $A \subset t U$ para todo $t>s_{U}$.

Proposição 2.3.23. Seja $X$ um espaço vetorial topológico e $K \subset X$ compacto. Então $K$ é um conjunto limitado.

Demonstração. Ver [Meg98] Proposição 2.2.11, página 170.

Definição 2.3.24. Sejam X um espaço vetorial topológico e $\left(x_{n}\right)_{n \in \mathbb{N}}$ uma sequência em $X$. Dizemos que $\left(x_{n}\right)_{n \in \mathbb{N}}$ é uma sequência de Cauchy se para toda vizinhança $U$ de 0 , existir um $N \in \mathbb{N}$ tal que $x_{n}-x_{m} \in U$ para todos $n, m \geq N$.

Proposição 2.3.25. Toda sequência de Cauchy em um espaço vetorial topológico é limitada.

Demonstração. Ver [Meg98] Proposição 2.2.12, página 171.

Definição 2.3.26 (Topologia Fraca). Seja X um espaço normado. A topologia fraca em X é a menor topologia para a qual todos funcionais $x^{*} \in X^{*}$ são contínuos com respeito a esta topologia. Denotamos a topologia fraca em $X$ por $\sigma\left(X, X^{*}\right)$.

Teorema 2.3.27. Um espaço normado X munido da topologia fraca é um espaço vetorial topológico localmente convexo.

Demonstração. Ver [Meg98] Teorema 2.5.2, página 212.

Observação 2.3.28. Uma base de abertos para a topologia fraca é a família de todos abertos do tipo

$$
V\left(x ; x_{1}^{*}, \ldots, x_{n}^{*}, \epsilon\right)=\left\{y \in X:\left|x_{i}^{*}(x)-x_{i}^{*}(y)\right|<\epsilon \text { para todo } i=1, \ldots, n\right\}
$$

onde $x \in X, x_{1}^{*}, \ldots, x_{n}^{*} \in X^{*} e \epsilon>0$.

Definição 2.3.29 (Topologia Fraca*). Sejam $X$ um espaço normado e $Q: X \rightarrow X^{* *}$ a inclusão canônica no espaço bidual de $X$. Então a topologia fraca* em $X^{*}$ é a menor topologia em $X^{*}$ para a qual todos funcionais $f \in Q(X)$ são contínuos com respeito a esta topologia. Denotamos a topologia fraca* em $X^{*}$ por $\sigma\left(X^{*}, X\right)$.

Teorema 2.3.30. Seja $X$ um espaço normado. Então $X^{*}$ munido da topologia fraca* é um espaço vetorial topológico localmente convexo. Além disso, se $X$ for um espaço reflexivo, as topologias fraca e fraca* em $X^{*}$ coincidem.

Demonstração. Ver [Meg98] Teorema 2.6.2, página 224.

Observação 2.3.31. Uma base de abertos para a topologia fraca* é a familia de todos abertos do tipo

$$
V\left(x^{*} ; x_{1}, \ldots, x_{n}, \epsilon\right)=\left\{y^{*} \in X^{*}:\left|x^{*}\left(x_{i}\right)-y^{*}\left(x_{i}\right)\right|<\epsilon \text { para todo } i=1, \ldots, n\right\},
$$

onde $x^{*} \in X^{*}, x_{1}, \ldots, x_{n} \in X$ e $\epsilon>0$. 
Teorema 2.3.32 (Banach-Alaoglu). Seja X um espaço normado. Então o subconjunto $B_{X^{*}}$ é fraco* compacto.

Demonstração. Ver [Meg98] Teorema 2.6.18, página 229.

Proposição 2.3.33. Sejam $X$ espaço normado e $Q: X \rightarrow X^{* *}$ a inclusão canônica. Então $Q$ é um homeomorfismo entre $\left(X, \sigma\left(X, X^{*}\right)\right)$ e $(Q(X), \tau)$, onde $\tau$ é a topologia de subespaço de $Q(X)$ com respeito à topologia $\sigma\left(X^{* *}, X^{*}\right)$ em $X^{* *}$.

Demonstração. Ver [Meg98] Proposição 2.6.24, página 232.

Corolário 2.3.34. Seja $X$ um espaço reflexivo. Então $B_{X}$ é compacto com respeito à topologia fraca de $X$.

Demonstração. Temos que $Q\left(B_{X}\right)=B_{X^{* *}}$, que é $\sigma\left(X^{* *}, X^{*}\right)$-compacto pelo Teorema 2.3.32. Assim, pela proposição $2.3 .33, B_{X}$ é um conjunto compacto com respeito à topologia fraca de $X$.

Os próximos dois resultados destoam um pouco do resto da seção mas serão usados no capítulo 4.

Teorema 2.3.35 (Ernest Michael). Sejam $X$ e $Y$ espaços de Banach e $T: X \rightarrow Y$ um operador linear contínuo e sobrejetor. Então para cada $\lambda>1$ existe uma função continua $\varphi: Y \rightarrow X$ satisfazendo

1. $\varphi(y) \in T^{-1}[\{y\}]$ para cada $y \in Y$.

2. $\|\varphi(y)\| \leq \lambda \inf \left\{\|z\|: z \in T^{-1}[\{y\}]\right\}$ para cada $y \in Y$.

3. $\varphi(\alpha y)=\alpha \varphi(y)$ para todo $\alpha \in \mathbb{F}$ e para todo $y \in Y$.

Demonstração. Ver [Mic56] Proposição 7.2, página 375.

Teorema 2.3.36 (Teorema do ponto fixo de Schauder). Sejam $X$ espaço de Banach e $K \subset X$ um compacto convexo. Então toda função contínua $f: K \rightarrow K$ tem um ponto fixo.

Demonstração. Ver [Sch30].

\subsubsection{Redes}

Como vamos trabalhar com espaços vetoriais topológicos, o conceito de rede é conveniente para verificar algumas propriedades topológicas, como compacidade.

Definição 2.3.37. Um conjunto dirigido é um conjunto $I \neq \emptyset$ com uma relação $\preceq$ satisfazendo:

1. $\alpha \preceq \alpha$ para todo $\alpha \in I$. 
2. $\alpha \preceq \beta$ e $\beta \preceq \gamma$ implicam $\alpha \preceq \gamma$ para todo $\alpha, \beta, \gamma \in I$.

3. para todos $\alpha, \beta \in I$, existe $\gamma_{\alpha, \beta} \in I$ tal que $\alpha \preceq \gamma_{\alpha, \beta}$ e $\beta \preceq \gamma_{\alpha, \beta}$.

Definição 2.3.38. Sejam $X$ um conjunto e I um conjunto dirigido. Uma rede em $X$ é uma função $f: I \rightarrow X$. Se $f(\alpha)=x_{\alpha}$ para cada $\alpha \in I$, podemos denotar $f$ por $\left(x_{\alpha}\right)_{\alpha \in I}$.

Quando não for necessário explicitar o conjunto de índices $I$, vamos omiti-lo para não sobrecarregar a notação.

Definição 2.3.39. Sejam $X$ um espaço topológico e $\left(x_{\alpha}\right)_{\alpha}$ uma rede em $X$. Dizemos que $\left(x_{\alpha}\right)_{\alpha}$ converge para $x \in X$ se para cada aberto $U$ de $X$ contendo $x$, existe $\alpha_{U} \in I$ tal que $\alpha_{U} \preceq \alpha$ implica $x_{\alpha} \in U$.

Proposição 2.3.40. Se $X$ é um espaço topológico Hausdorff, então o limite de uma rede $\left(x_{\alpha}\right)_{\alpha}$, caso exista, é único.

Demonstração. Ver Proposição 2.1.17 [Meg98], página 145.

Podemos denotar então o limite de uma rede convergente $\left(x_{\alpha}\right)_{\alpha}$ em um espaço topológico Hausdorff por $\lim _{\alpha} x_{\alpha}$.

O resultado a seguir caracteriza funções contínuas através de redes.

Proposição 2.3.41. Sejam $X$ e $Y$ espaços topológicos, $x_{0} \in X$ e $f: X \rightarrow Y$ uma função. Então $f$ é continua em $x_{0}$ se e somente se $\lim _{\alpha} f\left(x_{\alpha}\right)=f\left(x_{0}\right)$ para qualquer rede $\left(x_{\alpha}\right)_{\alpha}$ convergindo para $x_{0}$ em $X$.

Demonstração. Ver Proposição 2.1.21 [Meg98], página 146.

Para caracterizar conjuntos compactos por redes assim como caracterizamos compactos em espaços métricos com sequências, precisamos do conceito de subrede.

Definição 2.3.42. Sejam I um conjunto dirigido e $J \subset I$. Dizemos que $J$ é cofinal se para cada $\alpha \in I$, existe $\beta_{\alpha} \in J$ tal que $\alpha \preceq \beta_{\alpha}$.

Definição 2.3.43. Sejam $X$ um conjunto, $I$ um conjunto dirigido e $f: I \rightarrow X$ uma rede em $X$. Suponha ainda que $J$ é um outro conjunto dirigido e $g: J \rightarrow I$ uma função tal que

1. $g\left(\beta_{1}\right) \preceq g\left(\beta_{2}\right)$ para $\beta_{1} \preceq \beta_{2}$ em J.

2. $g[J]$ é cofinal em $I$.

Então a rede $f \circ g: J \rightarrow X$ é chamada de subrede de $f$.

Proposição 2.3.44. Sejam $X$ um espaço topológico e $K \subset X$. Então $K$ é compacto se e somente se toda rede em $K$ admite uma subrede com limite em $K$.

Demonstração. Ver [Meg98] Proposição 2.1.37, página 152. 
Assim como acontece com sequências, podemos caracterizar quando uma rede converge na topologia fraca ou fraca* de um espaço normado da seguinte forma:

Proposição 2.3.45. Seja X um espaço normado.

1. Uma rede $\left(x_{\alpha}\right)_{\alpha}$ em $X$ converge para $x \in X$ na topologia fraca se e somente se a rede em $\mathbb{F}$ dada por $\left(x^{*}\left(x_{\alpha}\right)\right)_{\alpha}$ converge para $x^{*}(x)$ para todo $x^{*} \in X^{*}$.

2. Uma rede $\left(x_{\alpha}^{*}\right)_{\alpha}$ em $X^{*}$ converge para $x^{*} \in X^{*}$ na topologia fraca* se e somente se a rede em $\mathbb{F}$ dada por $\left(x_{\alpha}^{*}(x)\right)_{\alpha}$ converge para $x^{*}(x)$ para todo $x \in X$.

Demonstração. Ver Proposição 2.4.4 [Meg98], página 204.

\subsubsection{Ultrafiltros e Ultraprodutos}

Agora iremos introduzir os conceitos de ultrafiltros e ultraprodutos. Com essas ferramentas em mãos, iremos obter diversos resultados a respeito de isomorfismos Lipschitz e homeomorfismos uniformes entre espaços de Banach, que serão definidos nos capítulos futuros.

Definição 2.3.46. Seja $\mathcal{I}$ um conjunto não vazio. Um filtro em $\mathcal{I}$ é um subconjunto $\mathcal{F}$ de $\mathcal{P}(\mathcal{I})$ satisfazendo:

1. $\emptyset \notin \mathcal{F}$.

2. $A \subset B$ e $A \in \mathcal{F}$, então $B \in \mathcal{F}$

3. Se $A, B \in \mathcal{F}$, então $A \cap B \in \mathcal{F}$.

Um ultrafiltro em $\mathcal{I}$ é um filtro $\mathcal{U}$ em $\mathcal{I}$ que é maximal, isto é, se $\mathcal{F}$ é um filtro em $\mathcal{I}$ contendo $\mathcal{U}$, então $\mathcal{F}=\mathcal{U}$.

Proposição 2.3.47. Sejam $\mathcal{I}$ um conjunto não vazio e $\mathcal{U}$ um filtro em $\mathcal{I}$. Então $\mathcal{U}$ é ultrafiltro se e somente se para todo $A \subset \mathcal{I}$, temos $A \in \mathcal{U}$ ou $A^{c} \in \mathcal{U}$, mas não ambos.

Demonstração. Suponha que $\mathcal{U}$ seja um filtro que não satisfaz a propriedade acima, isto é, existe $A \in \mathcal{I}$ tal que $A \notin \mathcal{U}$ e $A^{c} \notin \mathcal{U}$. Suponha sem perda de generalidade que $A \neq \emptyset$ (caso contrário consideramos $\mathcal{I}=A^{c}$ ). Veja que não podemos ter $A \cap B=\emptyset$ para algum $B \in \mathcal{U}$, pois teríamos $B \subset A^{c}$ e pela propriedade 2 de filtro, obteríamos $A^{c} \in \mathcal{U}$, uma contradição. Definimos então um conjunto $\mathcal{F}=\{C \subset \mathcal{I}: A \cap B \subset C$ para algum $B \in \mathcal{U}\}$. Note que $\emptyset \notin \mathcal{F}$ e que a propriedade 2 de filtro vale pela definição do conjunto. Se $C, D \in \mathcal{F}$, então existem $B_{C}$ e $B_{D}$ em $\mathcal{U}$ tais que $A \cap B_{C} \subset C$ e $A \cap B_{D} \subset D$. Mas então $A \cap\left(B_{C} \cap B_{D}\right) \subset C \cap D$ e temos que $B_{C} \cap B_{D} \in \mathcal{U}$ pois $\mathcal{U}$ é um filtro. Portanto $\mathcal{F}$ é um filtro contendo $\mathcal{U}$ e contendo $A$, logo $\mathcal{U}$ não é maximal, isto é, não é um ultrafiltro.

Por outro lado, suponha que $\mathcal{U}$ não seja um filtro maximal. Então existe um filtro $\mathcal{F}$ contendo 
$\mathcal{U}$ propriamente, isto é, existe $A \in \mathcal{F} \backslash \mathcal{U}$. Pelas propriedades 1 e 3 de filtro, não podemos ter $A^{c} \in \mathcal{U}$ pois caso contrário, $A^{c} \in \mathcal{F}$ e $\emptyset=A \cap A^{c} \in \mathcal{F}$. Portanto $A \notin \mathcal{U}$ e $A^{c} \notin \mathcal{U}$. Isto é, se para todo $A \subset \mathcal{I}$, tivermos $A \in \mathcal{U}$ ou $A^{c} \in \mathcal{U}$, devemos ter que $\mathcal{U}$ é um ultrafiltro.

Definição 2.3.48. Seja $\mathcal{U}$ um ultrafiltro em $\mathcal{I}$. Dizemos que $\mathcal{U}$ é principal em um ponto $x \in \mathcal{I}$ se $\{x\} \in \mathcal{U}$. Dizemos que $\mathcal{U}$ é não principal caso $\mathcal{U}$ não seja um ultrafiltro principal em nenhum ponto de $\mathcal{I}$.

Proposição 2.3.49. Sejam $\mathcal{I}$ um conjunto não vazio e $\mathcal{U}$ um ultrafiltro em $\mathcal{I}$. Temos:

1. $\mathcal{U}$ é um ultrafiltro principal em $x \in \mathcal{I}$, se e somente se $\mathcal{U}=\{A \subset \mathcal{I}: x \in A\}$.

2. Se $\mathcal{U}$ é não principal, então $\mathcal{U}$ não pode conter conjuntos finitos.

Demonstração. 1) Note que se $A \in \mathcal{U}$, necessariamente temos $x \in A$ pois caso contrário $\emptyset=A \cap\{x\} \in \mathcal{U}$. Logo $\mathcal{U} \subset\{A \subset \mathcal{I}: x \in A\}$ e a igualdade vale pois $\{A \subset \mathcal{I}: x \in A\}$ é um filtro contendo $\mathcal{U}$, que é maximal. Para a volta, basta provarmos que $\mathcal{U}$ é um filtro maximal. De fato, seja $\mathcal{F}$ é um filtro tal que $\mathcal{U} \subset \mathcal{F}$. Se $A \in \mathcal{F}$, temos que $\{x\} \cap A \in \mathcal{F}$ e pela propriedade 1) de filtro temos $x \in A$. Assim $\mathcal{F}=\mathcal{U}$.

2) Suponha por contradição que $A$ seja finito e $A \in \mathcal{U}$. Como $\mathcal{U}$ é não principal, para cada $a \in A$ temos que $\{a\} \notin \mathcal{U}$. Pela proposição anterior, $\{a\}^{c} \in \mathcal{U}$ para todo $a \in A$. Mas então $\emptyset=A \cap\left(\bigcap_{a \in A}\{a\}^{c}\right) \in \mathcal{U}$ pela propriedade 3 de filtro, obtendo uma contradição.

Definição 2.3.50. Seja X um espaço topológico Hausdorff, $\left(x_{n}\right)_{n \in \mathbb{N}}$ uma sequência em X e $\mathcal{U}$ um ultrafiltro não principal de $\mathbb{N}$. Dizemos que $x$ é o ultralimite de $\left(x_{n}\right)_{n \in \mathbb{N}}$ se para todo aberto $V$ contendo $x$, temos $\left\{n \in \mathbb{N}: x_{n} \in V\right\} \in \mathcal{U}$.

Observação 2.3.51. Sob as condições acima, afirmamos que o ultralimite de $\left(x_{n}\right)_{n \in \mathbb{N}}$, caso exista, é único. De fato, sejam $x$ e y ultralimites distintos da sequência acima. Como X é Hausdorff, existem abertos $U$ e $V$ tais que $x \in U, y \in V$ e $U \cap V=\emptyset$. Mas então o ultrafiltro $\mathcal{U}$ irá conter o conjunto $\left\{n \in \mathbb{N}: x_{n} \in U\right\} \cap\left\{n \in \mathbb{N}: x_{n} \in V\right\}=\emptyset$, o que não pode ocorrer em um filtro. Podemos então escrever o ultralimite da sequência $\left(x_{n}\right)_{n \in \mathbb{N}}$ como $\lim _{n \in \mathcal{U}} x_{n}$.

Também vale observar que sempre que existir o limite tradicional de uma sequência, este ponto será o ultralimite da sequência para qualquer ultrafiltro não principal $\mathcal{U}$ em $\mathbb{N}$. De fato, sejam $\left(x_{n}\right)_{n \in \mathbb{N}}$ umas sequência convergente e $x=\lim _{n \in \mathbb{N}} x_{n}$. Se $\mathcal{U}$ é um ultrafiltro não principal em $\mathbb{N}$, então para cada aberto $V$ contendo $x$, temos que $\left\{n \in \mathbb{N}: x_{n} \in V\right\}$ é um conjunto cofinito pela definição de limite de sequências. Portanto, pela Proposição 2.3.49 temos que $\left\{n \in \mathbb{N}: x_{n} \in V\right\}^{c} \notin \mathcal{U}$ e segue da Proposição 2.3.47 que $\left\{n \in \mathbb{N}: x_{n} \in V\right\} \in \mathcal{U}$.

Lema 2.3.52. Sejam $X$ e $Y$ espaços topológicos Hausdorff, $f: X \rightarrow Y$ contínua e $\left(x_{n}\right)_{n}$ uma sequência em $X$ cujo ultralimite $x=\lim _{n \in \mathcal{U}} x_{n}$ existe. Então $\lim _{n \in \mathcal{U}} f\left(x_{n}\right)$ existe em $Y$ e $\lim _{n \in \mathcal{U}} f\left(x_{n}\right)=f(x)$. 
Demonstração. Seja $V \subset Y$ um aberto contendo $f(x)$. Então, como $f$ é contínua, existe um aberto $U \subset X$ contendo $x$ tal que $y \in U$ implica $f(y) \in V$. Como $x=\lim _{n \in \mathcal{U}} x_{n}$, temos que $\left\{n \in \mathbb{N}: x_{n} \in U\right\} \in \mathcal{U}$. Mas pela escolha de $U$, temos

$$
\left\{n \in \mathbb{N}: x_{n} \in U\right\} \subset\left\{n \in \mathbb{N}: f\left(x_{n}\right) \in V\right\}
$$

Portanto $\left\{n \in \mathbb{N}: f\left(x_{n}\right) \in V\right\} \in \mathcal{U}$ e pela arbitrariedade de $V$, segue que $f(x)=\lim _{n \in \mathbb{N}} f\left(x_{n}\right)$.

Lema 2.3.53. Sejam $\mathcal{U}$ ultrafiltro não principal em $\mathbb{N},\left(x_{n}\right)$ e $\left(y_{n}\right)$ sequências reais cujo ultralimite existe e suponha que exista $D \in \mathcal{U}$ tal que $x_{n} \leq y_{n}$ para cada $n \in D$. Então $\lim _{n \in \mathcal{U}} x_{n} \leq \lim _{n \in \mathcal{U}} y_{n}$

Demonstração. Suponha que $\lim _{n \in \mathcal{U}} x_{n}>\lim _{n \in \mathcal{U}} y_{n}$. Sejam $(a, b)$ e $(c, d)$ intervalos contendo $\lim _{n \in \mathcal{U}} y_{n}$ e $\lim _{n \in \mathcal{U}} x_{n}$, respectivamente, e tais que $b<c$. Então tomamos $D_{0}=\left\{n \in \mathbb{N}: y_{n} \in(a, b)\right\} \cap\{n \in$ $\left.\mathbb{N}: x_{n} \in(c, d)\right\}$. Temos que $D_{0} \in \mathcal{U}$ pois é a interseção de dois elementos de $\mathcal{U}$ e $\operatorname{logo}$ $D_{0} \cap D \neq \emptyset$, que é uma contradição pois existiria $n \in D$ tal que $x_{n}>y_{n}$.

Lema 2.3.54. Sejam $\left(x_{n}\right)_{n \in \mathbb{N}}$ uma sequência em um espaço topológico $K$ compacto e Hausdorff e $\mathcal{U}$ um ultrafiltro não principal. Então existe o ultralimite $\lim _{n \in \mathcal{U}} x_{n}$.

Demonstração. Suponha por contradição que não exista o ultralimite. Então para todo $x \in$ $K$, existe um $V_{x}$ aberto contendo $x$ tal que $\left\{n \in \mathbb{N}: x_{n} \in V_{x}\right\} \notin \mathcal{U}$, isto é, $D_{x}=\{n \in \mathbb{N}$ : $\left.x_{n} \notin V_{x}\right\} \in \mathcal{U}$. Por compacidade, existem $y_{1}, \ldots, y_{m} \in K$ tais que $K=\bigcup_{i=1}^{m} V_{y_{i}}$. Então para todo $n \in \mathbb{N}$, existe $1 \leq i \leq m$ tal que $x_{n} \in V_{y_{i}}$, isto é, $n \notin D_{y_{i}}$ e portanto $\emptyset=\bigcap_{i=1}^{m} D_{y_{i}} \in \mathcal{U}$, uma contradição.

Definição 2.3.55. Seja $X$ um espaço de Banach. Definimos $\ell_{\infty}(X)$ como sendo o espaço de todas sequências limitadas em $X$ munido da norma $\left\|\left(x_{n}\right)_{n \in \mathbb{N}}\right\|=\sup _{n}\left\|x_{n}\right\|$.

Observação 2.3.56. Se $X$ é um espaço de Banach, então $\ell_{\infty}(X)$ é um espaço de Banach.

Definição 2.3.57. Sejam $X$ um espaço de Banach e $\mathcal{U}$ um ultrafiltro em $\mathbb{N}$. Definimos $c_{0, \mathcal{U}}(X)$ como sendo o subespaço de $\ell_{\infty}(X)$ dado por $c_{0, \mathcal{U}}(X)=\left\{\left(x_{n}\right)_{n \in \mathbb{N}} \in \ell_{\infty}(X): \lim _{n \in \mathcal{U}} x_{n}=\right.$ $0\}$.

Lema 2.3.58. Sejam $X$ um espaço de Banach e $\mathcal{U}$ um ultrafiltro em $\mathbb{N}$. Então $c_{0, \mathcal{U}}(X)$ é um subespaço fechado de $\ell_{\infty}(X)$.

Demonstração. Vamos mostrar que $c_{0, \mathcal{U}}(X)$ é fechado. De fato, sejam $\left(x_{n}\right)_{n \in \mathbb{N}}$ uma sequência em $c_{0, \mathcal{U}}(X)$ convergente a $x \in \ell_{\infty}(X)$ e $\epsilon>0$. Queremos mostrar que $D_{0}=\{k \in \mathbb{N}: x(k) \in$ $(-\epsilon, \epsilon)\} \in \mathcal{U}$. Seja $N \in \mathbb{N}$ tal que $\left\|x-x_{N}\right\|<\epsilon / 2$. Sabemos que $D_{1}=\left\{k \in \mathbb{N}: x_{N}(k) \in\right.$ $(-\epsilon / 2, \epsilon / 2)\} \in \mathcal{U}$ pois $x_{N} \in c_{0, \mathcal{U}}(X)$. Veja que se $k \in D_{1}$, então

$$
|x(k)| \leq\left|x_{k}-x_{N}(k)\right|+\left|x_{N}(k)\right| \leq\left\|x-x_{N}\right\|+\left|x_{N}(k)\right|<\epsilon,
$$


$\log D_{1} \subset D_{0}$ e portanto $D_{0} \in \mathcal{U}$.

Definição 2.3.59. Sejam $X$ um espaço de Banach e $\mathcal{U}$ um ultrafiltro em $\mathbb{N}$. O ultraproduto de $X$ com respeito ao ultrafiltro $\mathcal{U}$ é o quociente $\ell_{\infty}(X) / c_{0, \mathcal{U}}(X)$ e o denotamos por $X_{\mathcal{U}}$.

Observação 2.3.60. Note que $X_{\mathcal{U}}$ é um espaço de Banach pelo Lema 2.3.58.

Proposição 2.3.61. Seja X um espaço de Banach. Então a norma $\|\cdot\|_{\mathcal{U}}$ em $X_{\mathcal{U}}$ pode ser dada por

$$
\|\bar{x}\|_{\mathcal{U}}=\lim _{n \in \mathcal{U}}\left\|x_{n}\right\|
$$

para cada $\bar{x} \in X_{\mathcal{U}} e\left(x_{n}\right)_{n \in \mathbb{N}} \in \bar{x}$.

Demonstração. Seja $\bar{x} \in X_{\mathcal{U}}$ e $x=\left(x_{n}\right)_{n \in \mathbb{N}} \in \bar{x}$. Seja $L=\lim _{n \in \mathcal{U}}\left\|x_{n}\right\|$, que existe pelo Lema 2.3.54. Queremos mostrar que $L=\inf _{z \in \bar{x}}\|z\|_{\infty}$. Seja $\epsilon>0$. Então $D=\left\{n \in \mathbb{N}:\left|\left\|x_{n}\right\|-L\right|<\right.$ $\epsilon\} \in \mathcal{U}$ por definição de ultralimite. Definimos uma sequência $y=\left(y_{n}\right)_{n \in \mathbb{N}}$ em $\ell_{\infty}(X)$ por

$$
y(n)= \begin{cases}0 & n \in D \\ x(n) & n \notin D\end{cases}
$$

Então $\lim _{n \in \mathcal{U}} y(n)=0$ e $\operatorname{logo} x-y \in \bar{x}$. Além disso, como $x_{n}-y_{n}=0$ se $n \notin D$ e $x_{n}-y_{n}=x_{n}$ se $n \in D$, segue que

$$
\inf _{z \in \bar{x}}\|z\|_{\infty} \leq\|x-y\|_{\infty}=\sup _{n \in \mathbb{N}}\left\|x_{n}-y_{n}\right\|=\sup _{n \in D}\left\|x_{n}\right\| \leq L+\epsilon
$$

Como $\epsilon$ é arbitrário, segue que $\inf _{z \in \bar{x}}\|z\|_{\infty} \leq L$.

Por outro lado, sejam $\epsilon>0$ e $y=\left(y_{n}\right)_{n \in \mathbb{N}}$ em $\ell_{\infty}(X)$ tal que $\lim _{n \in \mathcal{U}} y_{n}=0$. Considere

$$
D=\left\{n \in \mathbb{N}:\left\|y_{n}\right\|<\epsilon\right\} \cap\left\{n \in \mathbb{N}:\left|\left\|x_{n}\right\|-L\right|<\epsilon\right\}
$$

Veja que $D \in \mathcal{U}$ pois é uma interseção de elementos de $\mathcal{U}$ e logo existe $k \in D$. Mas então

$$
\|x-y\|_{\infty} \geq\left\|x_{k}-y_{k}\right\| \geq\left\|x_{k}\right\|-\left\|y_{k}\right\| \geq L-\epsilon-\epsilon=L-2 \epsilon .
$$

Como $y$ foi escolhido arbitrariamente, segue que

$$
\inf _{z \in \bar{x}}\|z\|_{\infty}=\inf _{w \in c_{0, \mathcal{U}}}\|x-w\|_{\infty} \geq\|x-y\|_{\infty} \geq L-2 \epsilon
$$

Como $\epsilon$ foi escolhido arbitrariamente, concluimos então que $\inf _{z \in \bar{x}}\|z\|_{\infty} \geq L$ e portanto vale a igualdade.

Proposição 2.3.62. Sejam X um espaço de Banach reflexivo e $\mathcal{U}$ um ultrafiltro não principal em $\mathbb{N}$. Então $X$ é isométrico a um subespaço complementado de $X_{\mathcal{U}}$. 
Demonstração. Podemos identificar $X$ com um subespaço de $X_{\mathcal{U}}$ através da função $I: X \rightarrow$ $X_{\mathcal{U}}$ dada por $I(x)=\overline{\left(x_{n}\right)_{n \in \mathbb{N}}}$, onde $x_{n}=x$ para todo $n \in \mathbb{N}$. Vamos mostrar que $I[X]$ é complementado em $X_{\mathcal{U}}$. Como $X$ é reflexivo, para todo $\left(x_{n}\right)_{n \in \mathbb{N}} \in \ell_{\infty}(X)$, existe o ultralimite $w-\lim _{n \in \mathcal{U}} x_{n}$ na topologia fraca de $X$. Note que se $\left(y_{n}\right)_{n \in \mathbb{N}} \in \overline{\left(x_{n}\right)_{n \in \mathbb{N}}}$, temos $\lim _{n \in \mathcal{U}}\left(x_{n}-y_{n}\right)=0$ e então $w-\lim _{n \in \mathcal{U}}\left(x_{n}-y_{n}\right)=0$. Podemos definir então a função $P: X_{\mathcal{U}} \rightarrow X$ por

$$
P\left(\overline{\left(x_{n}\right)_{n \in \mathbb{N}}}\right)=w-\lim _{n \in \mathcal{U}} x_{n} \text { para cada } \overline{\left(x_{n}\right)_{n \in \mathbb{N}}} \in X_{\mathcal{U}}
$$

É direto que $P$ é linear. Além disso, se $\overline{\left(x_{n}\right)_{n \in \mathbb{N}}} \in X_{\mathcal{U}}$, tome $x^{*} \in S_{X^{*}}$ tal que $x^{*}\left(P\left(\overline{\left(x_{n}\right)_{n \in \mathbb{N}}}\right)\right)=$ $\left\|P\left(\overline{\left(x_{n}\right)_{n \in \mathbb{N}}}\right)\right\|$. Então aplicando o Lema 2.3.52, temos

$$
\begin{aligned}
\left\|P\left(\overline{\left(x_{n}\right)_{n \in \mathbb{N}}}\right)\right\| & =\left|x^{*}\left(P\left(\overline{\left(x_{n}\right)_{n \in \mathbb{N}}}\right)\right)\right| \\
& =\left|x^{*}\left(w-\lim _{n \in \mathcal{U}} x_{n}\right)\right| \\
& =\left|\lim _{n \in \mathcal{U}} x^{*}\left(x_{n}\right)\right| \\
& =\lim _{n \in \mathcal{U}}\left|x^{*}\left(x_{n}\right)\right| \\
& \leq \lim _{n \in \mathcal{U}}\left\|x_{n}\right\| \\
& =\left\|\overline{\left(x_{n}\right)_{n \in \mathbb{N}}}\right\| .
\end{aligned}
$$

Portanto $P$ é um operador linear com norma 1. Por fim, se $x \in X$, então

$$
P(I(x))=w-\lim _{n \in \mathcal{U}} x=x
$$

\subsubsection{Propriedades locais de Espaços de Banach}

Neste seção, o conceito de estrutura local se refere a estrutura dos subespaços de dimensão finita de um espaço de Banach. Nossa referência principal será [AK98].

Definição 2.3.63. Uma sequência de Rademacher é uma sequência $\left(\xi_{n}\right)_{n \in \mathbb{N}}$ de variáveis aleatórias mutualmente independentes definidas em um espaço de probabilidade $(\Omega, \mathcal{F}, \mathbb{P})$ tais que $\mathbb{P}\left(\xi_{n}=1\right)=\mathbb{P}\left(\xi_{n}=-1\right)=\frac{1}{2}$.

Para não pesar a notação, vamos estabelecer $\epsilon_{k}= \pm 1$ nos índices sempre que estiver estabelecido um $n \in \mathbb{N}$ e quisermos percorrer o conjunto das $n$-uplas $\left\{\left(\epsilon_{1}, \ldots, \epsilon_{n}\right): \epsilon_{i}= \pm 1\right\}$.

Proposição 2.3.64. Sejam $\left(\xi_{k}\right)_{k}$ umas sequência de Rademacher, $X$ um espaço de Banach e $x_{1}, \ldots, x_{n} \in X$. Então

$$
\int_{\Omega}\left\|\sum_{k=1}^{n} \xi_{k}(\omega) x_{k}\right\| d \omega=\frac{1}{2^{n}} \sum_{\epsilon_{k}= \pm 1}\left\|\sum_{k=1}^{n} \epsilon_{k} x_{k}\right\| .
$$


Ou seja, a primeira integral é a média do conjunto $\left\{\left\|\sum_{k=1}^{n} \epsilon_{k} x_{k}\right\|:\left(\epsilon_{1}, \ldots, \epsilon_{n}\right) \in\{-1,1\}^{n}\right\}$.

Demonstração. Vejamos primeiro que

$$
\Omega=\bigcup_{\epsilon_{k}= \pm 1}\left(\bigcap_{k=1}^{n}\left(\xi_{k}=\epsilon_{k}\right)\right) \cup N
$$

onde $N$ tem medida nula. De fato, se $\omega \notin \bigcup_{\epsilon_{k}= \pm 1}\left(\bigcap_{k=1}^{n}\left(\xi_{k}=\epsilon_{k}\right)\right)$, existe $1 \leq k \leq n$ tal que $\xi_{k}(\omega) \notin\{-1,1\}$ e assim $N \subset \bigcup_{k=1}^{n} \xi_{k}^{-1}[\mathbb{R} \backslash\{-1,1\}]$, que é uma união finita de conjuntos de medida nula pela definição de sequência de Rademacher. Mas então

$$
\begin{aligned}
\int_{\Omega}\left\|\sum_{k=1}^{n} \xi_{k}(\omega) x_{k}\right\| d \omega & =\sum_{\epsilon_{k}= \pm 1} \int_{\bigcap_{k=1}^{n}\left(\xi_{k}=\epsilon_{k}\right)}\left\|\sum_{k=1}^{n} \xi_{k}(\omega) x_{k}\right\| d \omega \\
& =\sum_{\epsilon_{k}= \pm 1} \int_{\bigcap_{k=1}^{n}\left(\xi_{k}=\epsilon_{k}\right)}\left\|\sum_{k=1}^{n} \epsilon_{k} x_{k}\right\| d \omega \\
& =\sum_{\epsilon_{k}= \pm 1} \mathbb{P}\left(\bigcap_{k=1}^{n}\left(\xi_{k}=\epsilon_{k}\right)\right)\left\|\sum_{k=1}^{n} \epsilon_{k} x_{k}\right\|
\end{aligned}
$$

Como as variáveis aleatórias são independentes, temos que

$$
\mathbb{P}\left(\bigcap_{k=1}^{n}\left(\xi_{k}=\epsilon_{k}\right)\right)=\prod_{k=1}^{n} \mathbb{P}\left(\left(\xi_{k}=\epsilon_{k}\right)\right)=\frac{1}{2^{n}}
$$

e segue resultado.

Definição 2.3.65. Seja $\left(\xi_{k}\right)_{k}$ umas sequência de Rademacher. Um espaço de Banach X tem tipo de Rademacher p para algum $1 \leq p \leq 2$ se existir uma constante $C$ tal que para cada sequência finita $x_{1}, \ldots, x_{n} \in X$, temos

$$
\left(\mathbb{E}\left\|\sum_{i=1}^{n} \xi_{i} x_{i}\right\|^{p}\right)^{1 / p} \leq C\left(\sum_{i=1}^{n}\left\|x_{i}\right\|^{p}\right)^{1 / p} .
$$

De forma análoga, um espaço de Banach $X$ tem cotipo de Rademacher q para algum $2 \leq$ $q \leq \infty$ se existir uma constante $C$ tal que para cada sequência finita $x_{1}, \ldots, x_{n} \in X$, temos

$$
\left(\sum_{i=1}^{n}\left\|x_{i}\right\|^{q}\right)^{1 / q} \leq C\left(\mathbb{E}\left\|\sum_{i=1}^{n} \xi_{i} x_{i}\right\|^{q}\right)^{1 / q} .
$$

\section{Exemplo 2.3.66.}

1. Pela desigualdade triangular, todo espaço de Banach tem tipo 1 e cotipo $\infty$; 
2. Todo espaço de Hilbert tem tipo e cotipo 2.

3. Se $1 \leq p \leq 2$, então $L_{p}(\mu)$ tem tipo $p$ e cotipo 2 .

4. Se $2<q<\infty$, então $L_{q}(\mu)$ tem tipo 2 e cotipo $q$.

Demonstração. 2) Segue da Lei do paralelogramo generalizada, veja [AK98] Proposição 6.2.9. Para 3) e 4), indicamos [AK98] Teorema 6.2.14.

O conceito de representabildiade finita é usado para dizer quando dois espaços de Banach têm a mesma estrutura local.

Definição 2.3.67. Sejam $X$ e $Y$ dois espaços de Banach isomorfos. Definimos a distância de Banach-Mazur por $d(X, Y)=\inf \left\{\|T\|\left\|T^{-1}\right\|: T: X \rightarrow Y\right.$ é um isomorfismo $\}$.

Definição 2.3.68. Sejam $X$ e $Y$ espaços de Banach de dimensão infinita. Dizemos que $X$ é finitamente representável em $Y$ se para qualquer subespaço $E \subset X$ de dimensão finita $e$ $\epsilon>0$, existe um subspaço de dimensão finita $F$ de $Y$ com $\operatorname{dim} F=\operatorname{dim} E$ e $d(E, F)<1+\epsilon$.

\section{Observação 2.3.69.}

1. Todo subespaço $Y$ de dimensão infinita de um espaço de Banach $X$ de dimensão infinita é finititamente representável $X$.

2. Essa propriedade é transitiva: ou seja, se $X, Y$ e $Z$ são espaços de Banach de dimensão infinita tais que $X$ é finitamente representável em $Y$ e $Y$ é finitamente representável em $Z$, então $X$ é finitamente representável em $Z$.

Definição 2.3.70. Sejam $1 \leq p \leq \infty$ e $n \in \mathbb{N}$. Definimos o espaço $\ell_{p}^{n}$ como sendo o espaço $\mathbb{R}^{n}$ munido da norma $\|\cdot\|_{p}$. Definimos ainda o espaço $\ell_{\infty}^{n}$ como sendo $\mathbb{R}^{n}$ munido da norma $\|\cdot\|_{\infty}$

Exemplo 2.3.71. Todo espaço de Banach é finitamente representável em $c_{0}$.

Demonstração. Sejam $X$ um espaço de Banach e $E$ um subespaço de $X$ de dimensão finita. Sejam $\epsilon>0$ arbitrário e $\nu<1$ tal que $\frac{1}{1-\nu}<1+\epsilon$. Tome $e_{1}^{*}, \ldots, e_{n}^{*} \in B_{E^{*}}$ tais que $B_{E^{*}} \subset \bigcup_{i=1}^{n} B\left(e_{i}^{*} ; \nu\right)$. Definimos então $T: E \rightarrow \ell_{\infty}^{n}$ por $T(e)=\left(e_{i}^{*}(e)\right)_{i=1}^{n}$. Veja que

$$
\|T\|=\sup _{e \in B_{E}}\|T(e)\|=\sup _{e \in B_{E}}\left(\max _{i=1, \ldots, n}\left|e_{i}^{*}(e)\right|\right) \leq\|e\| \leq 1
$$

pois cada $e_{i}^{*} \in B_{E^{*}}$, assim $\|T\| \leq 1$. Seja $e \in S_{E}$. Pelo Corolário 2.3.6, existe $e^{*} \in S_{E}^{*}$ tal que $e^{*}(e)=1$. Seja $i_{0} \in\{1, \ldots, n\}$ tal que $\left\|e^{*}-e_{i_{0}}^{*}\right\|<\nu$. Temos então que

$$
\left|e^{*}(e)\right|-\left|e_{i_{0}}^{*}(e)\right| \leq\left|e^{*}(e)-e_{i_{0}}^{*}(e)\right| \leq\left\|e^{*}-e_{i_{0}}^{*}\right\|<\nu
$$


e assim

$$
\|T(e)\|=\max _{i=1, \ldots, n}\left|e_{i}^{*}(e)\right| \geq\left|e_{i_{0}}^{*}(e)\right|>1-\nu .
$$

Temos assim que $T$ é um isomorfismo na imagem e que $\left\|T^{-1}\right\| \leq \frac{1}{1-\nu}$. Portanto

$$
\|T\|\left\|T^{-1}\right\| \leq \frac{1}{1-\nu}<1+\epsilon .
$$

Lema 2.3.72. Sejam X um espaço de Banach e E um espaço normado de dimensão finita. Suponha que $x_{1}, \ldots, x_{n} \in E$ sejam tais que $S_{E} \subset \bigcup_{i=1}^{n} B\left(x_{i}, \epsilon\right)$, onde $0<\epsilon<1$. Suponha que $T: E \rightarrow X$ seja uma transformação linear satisfazendo $1-\epsilon \leq\left\|T\left(x_{i}\right)\right\| \leq 1+\epsilon$ para $1 \leq i \leq n$. Então para todo $e \in E$ temos

$$
\frac{1-3 \epsilon}{1-\epsilon}\|e\| \leq\|T(e)\| \leq \frac{1+\epsilon}{1-\epsilon}\|e\|
$$

Demonstração. Sejam $e \in E$ tal que $\|e\|=1$ e $x_{j}$ tal que $\left\|e-x_{j}\right\| \leq \epsilon$. Então

$$
\|T(e)\| \leq\left\|T(e)-T\left(x_{j}\right)\right\|+\left\|T\left(x_{j}\right)\right\| \leq\|T\| \epsilon+(1+\epsilon) .
$$

Logo

$$
\|T\| \leq\|T\| \epsilon+(1+\epsilon)
$$

e assim $\|T\| \leq \frac{1+\epsilon}{1-\epsilon}$. Por outro lado,

$$
\|T(e)\| \geq\left\|T\left(x_{j}\right)\right\|-\left\|T(e)-T\left(x_{j}\right)\right\| \geq(1-\epsilon)-\|T\| \epsilon \geq(1-\epsilon)-\frac{1+\epsilon}{1-\epsilon} \epsilon=\frac{1-3 \epsilon}{1-\epsilon} .
$$

Proposição 2.3.73. Sejam X e Y espaços de Banach de dimensão infinita. Então

1. $X_{\mathcal{U}}$ é finitamente representável em $X$ para todo ultrafiltro não principal $\mathcal{U}$ em $\mathbb{N}$;

2. Se $Y$ é separável, então $Y$ é finitamente representável em $X$ se e somente se $Y$ é isométrico a um subespaço de $X_{\mathcal{U}}$.

Demonstração. 1) Fixemos representantes $x \in \bar{x}$ para todo $\bar{x} \in X_{\mathcal{U}}$. Sejam $E$ um subespaço de $X_{\mathcal{U}}$ de dimensão finita, $\nu>0$ e $\bar{x}_{1}, \ldots, \bar{x}_{n} \in S_{E}$ tais que $S_{E} \subset \bigcup_{i=1}^{n} B\left(\bar{x}_{i} ; \nu\right)$. Para cada $i \in\{1 \ldots, n\}$, temos que

$$
1=\left\|\bar{x}_{i}\right\|_{X_{\mathcal{U}}}=\lim _{n \in \mathcal{U}}\left\|x_{i}(n)\right\|
$$

$\operatorname{logo}$ existe um $A_{i} \in \mathcal{U}$ tal que $1-\nu<\left\|x_{i}(n)\right\|<1+\nu$ para cada $n \in A_{i}$. Tomamos então $A=\bigcap_{i=1}^{n} A_{i} \in \mathcal{U}$. Seja $k \in A$ qualquer. Definimos então $T: E \rightarrow X$ por $T(\bar{x})=x(k)$. Note que

$$
1-\nu<\left\|T\left(\bar{x}_{i}\right)\right\|=\left\|x_{i}(k)\right\|<1+\nu .
$$


Pelo Lema acima, $\|T\|\left\|T^{-1}\right\|<1+\epsilon$ desde que $\nu$ seja escolhido de forma que $\frac{1+\nu}{1-3 \nu}<1+\epsilon$.

2) Suponha que $Y$ seja finitamente representável em $X$. Vamos provar que $Y$ é isométrico a um subespaço de $X_{\mathcal{U}}$. Seja $\left(E_{n}\right)_{n \in \mathbb{N}}$ uma sequência crescente de subespaços de $Y$ de dimensão finita cuja união $E=\bigcup_{n \in \mathbb{N}} E_{n}$ é densa em $Y$. Para cada $n \in \mathbb{N}$, seja $T_{n}: E_{n} \rightarrow X$ tal que $1-1 / n \leq\left\|T_{n}\right\| \leq 1$. Definimos então $L: E \rightarrow \ell_{\infty}(X)$ por

$$
L(e)(k)= \begin{cases}0 & e \notin E_{k} \\ T_{k}(e) & e \in E_{k}\end{cases}
$$

Note que $L$ não é necessariamente linear, mas $L(x+y)-L(x)-L(y)$ é zero a partir de um certo $k$. De fato, se $x \in E_{k}$ e $y \in E_{k^{\prime}}$, seja $l$ o máximo dos dois. Então $x, y$ e $x+y$ pertencem ao espaço $E_{l}$ e $\operatorname{logo}$

$$
L(x+y)(n)=T_{n}(x+y)=T_{n}(x)+T_{n}(y)=L(x)(n)+L(y)(n)
$$

para todo $n \geq l$. Se $q: \ell_{\infty}(X) \rightarrow X_{\mathcal{U}}$ é a função quociente, então $q \circ L: E \rightarrow X_{\mathcal{U}}$ é um operador linear bem definido. Vamos provar que é uma isometria: seja $e \in E$ e $\epsilon>0$. Seja $k$ suficientemente grande tal que $e \in E_{k}$ e $1 / k<\epsilon$. Então para todo $n \geq k$, temos

$$
(1-\epsilon)\|e\|<(1-1 / k)\|e\| \leq(1-1 / n)\|e\| \leq\left\|T_{n}(e)\right\| \leq\|e\| .
$$

Assim, como $\|q \circ L(e)\|=\lim _{k \in \mathcal{U}}\left\|T_{k}(e)\right\|$, segue que $\|q \circ L(e)\|=\|e\|$.

Por outro lado, se $Y$ é isométrico a um subespaço de $X_{\mathcal{U}}$, segue de 1) que $Y$ é finitamente representável em $X$.

O teorema abaixo nos diz que, de certo modo, todo espaço é reflexivo localmente, isto é, a estrutura local do bidual de um espaço é a mesma do espaço em questão.

Teorema 2.3.74 (Princípio da Reflexividade Local). Seja X um espaço de Banach. Sejam $F$ um subespaço de dimensão finita de $X^{* *}$ e $G$ um subespaço de dimensão finita de $X^{*}$. Então dado $\epsilon>0$, existem um subespaço $E$ de $X$ contendo $F \cap X$ com dimE $=\operatorname{dim} F e$ um isomorfismo linear $T: F \rightarrow E$ tal que $\|T\|\left\|T^{-1}\right\|<1+\epsilon$ e satisfazendo $T(x)=x$ para cada $x \in F \cap X$ e $x^{*}\left(T\left(x^{* *}\right)\right)=x^{* *}\left(x^{*}\right)$ para cada $x^{*} \in G$ e $x^{* *} \in F$. Em particular, $X^{* *} e ́$ finitamente representável em $X$.

Demonstração. Indicamos [AK98] Teorema 11.2.4.

Definição 2.3.75. Dizemos que um espaço de Banach $X$ é superreflexivo se todo espaço de Banach $Y$ finitamente representável em $X$ é reflexivo.

Definição 2.3.76. Seja $1 \leq p \leq \infty$. Um espaço de Banach $X$ é chamado de espaço $\mathcal{L}_{p}$ se existe uma constante $\lambda>1$ tal que para todo subespaço de dimensão finita $E$ de $X$, existe um subespaço $F$ de $X$ contendo $E$ tal que $d\left(F, \ell_{p}^{m}\right) \leq \lambda$, onde $m=\operatorname{dim} F$. 
Teorema 2.3.77. Seja $1<p<\infty$ com $p \neq 2$. Então um espaço de Banach $X$ é um espaço $\mathcal{L}_{p}$ se e somente se $X$ é isomorfo a um subespaço complementado de $L_{p}$ e não é isomorfo a um espaço de Hilbert.

Demonstração. Indicamos [JL69].

Teorema 2.3.78. Sejam $1 \leq p<\infty$ e X um espaço $\mathcal{L}_{p}$. Então $X_{\mathcal{U}}$ é um espaço $\mathcal{L}_{p}$.

Demonstração. Veja [Bou83] Proposição 1.22.

Lema 2.3.79. Sejam $X$ e $Y$ espaços de Banach. Suponha que $Y$ seja finitamente representável em $X$ e que $X$ tem tipo $p$ e cotipo $q$ para $1 \leq p \leq 2$ e $2 \leq q \leq \infty$. Então $Y$ tem tipo $p$ e cotipo $q$ com as mesmas constantes de $X$.

Demonstração. Seja $\left(\xi_{k}\right)_{k}$ umas sequência de Rademacher. Como $X$ tem tipo $p$, existe uma constante $C$ tal que para cada sequência finita $x_{1}, \ldots, x_{n} \in X$, temos

$$
\left(\mathbb{E}\left\|\sum_{i=1}^{n} \xi_{i} x_{i}\right\|^{p}\right)^{1 / p} \leq C\left(\sum_{i=1}^{n}\left\|x_{i}\right\|^{p}\right)^{1 / p} .
$$

Vamos mostrar que $Y$ tem tipo $p$. Sejam $y_{1}, \ldots, y_{n} \in Y$ distintos. Sejam $E$ o espaço gerado por $\left\{y_{1}, \ldots, y_{n}\right\}, F$ um subespaço de $X$ com $\operatorname{dim} F=\operatorname{dim} E$ e $T: E \rightarrow F$ isomorfismo tal que $\|T\|\left\|T^{-1}\right\|<1+\epsilon$. Então

$$
\begin{aligned}
\left(\mathbb{E}\left\|\sum_{i=1}^{n} \xi_{i} y_{i}\right\|^{p}\right)^{1 / p} & =\left(\int_{\Omega}\left\|\sum_{i=1}^{n} \xi_{i}(\omega) y_{i}\right\|^{p} d \mu(\omega)\right)^{1 / p} \\
& =\left(\int_{\Omega}\left\|T^{-1} \circ T\left(\sum_{i=1}^{n} \xi_{i}(\omega) x_{i}\right)\right\|^{p} d \mu(\omega)\right)^{1 / p} \\
& \leq\left\|T^{-1}\right\|\left(\int_{\Omega}\left\|\sum_{i=1}^{n} \xi_{i}(\omega) T\left(y_{i}\right)\right\|^{p} d \mu(\omega)\right)^{1 / p} \\
& \leq\left\|T^{-1}\right\| C\left(\sum_{i=1}^{n}\left\|T\left(y_{i}\right)\right\|^{p}\right)^{1 / p} \\
& \leq\|T\|\left\|T^{-1}\right\| C\left(\sum_{i=1}^{n}\left\|y_{i}\right\|^{p}\right)^{1 / p} \\
& <(1+\epsilon) C\left(\sum_{i=1}^{n}\left\|y_{i}\right\|^{p}\right)^{1 / p} .
\end{aligned}
$$

Como $\epsilon$ foi escolhido arbitrariamente, segue que

$$
\left(\mathbb{E}\left\|\sum_{i=1}^{n} \xi_{i} y_{i}\right\|^{p}\right)^{1 / p} \leq C\left(\sum_{i=1}^{n}\left\|y_{i}\right\|^{p}\right)^{1 / p} .
$$

A demonstração que $Y$ tem cotipo $q$ é análoga. 


\section{Capítulo 3}

\section{FUNÇÕES LIPSCHITZ ENTRE ESPAÇOS DE BANACH}

Vamos iniciar o capítulo com a definição de função lipschitziana.

Definição 3.0.1. Sejam $\left(M_{1}, d_{1}\right)$ e $\left(M_{2}, d_{2}\right)$ espaços métricos e $f: M_{1} \rightarrow M_{2}$. Dizemos que $f$ é uma função lipschitziana, ou simplesmente Lipschitz, se existe $A>0$ tal que $d_{2}(f(x), f(y)) \leq A d_{1}(x, y)$ para todos $x, y \in M_{1}$. Definimos ainda

$$
\operatorname{Lip}(f)=\sup \left\{\frac{d_{2}(f(x), f(y))}{d_{1}(x, y)}: x, y \in M_{1}, x \neq y\right\}
$$

A seguir, vamos definir uma classe de funções entre espaços métricos mais abrangente que as isometrias, porém bastante relevante para nosso estudo.

Definição 3.0.2. Sejam $M_{1}$ e $M_{2}$ espaços métricos. Dizemos que $M_{1}$ e $M_{2}$ são Lipschitz isomorfos se existir uma função invertivel $f: M_{1} \rightarrow M_{2}$ Lipschitziana tal que sua inversa $f^{-1}$ é Lipschitziana. Neste caso, dizemos que $f$ é um isomorfismo Lipschitz.

Pelo Teorema de Mazur-Ulam A.0.3, sabe-se que isometrias sobrejetoras entre espaços de Banach reais são funções afim. Assim, isometrias preservam toda estrutura linear dos espaços de Banach reais. O passo natural é estudar os isomorfismos Lipschitz e nos perguntar sob quais condições conseguimos resultados semelhantes. Indicamos como referências [BL00] e [Kal08].

O primeiro objetivo do capítulo vai ser demonstrar uma generalização do teorema de Rademacher abaixo para espaços de Banach.

Teorema 3.0.3 (Rademacher). Seja $f: \mathbb{R}^{n} \rightarrow \mathbb{R}^{m}$ uma função Lipschitz. Então $f$ é diferenciável quase sempre com respeito à medida de Lebesgue.

Demonstração. Indicamos [EG92], seção 3.1.2. 
Sabe-se de [Haa33] que dado um grupo topológico localmente compacto $G$, existe uma única medida regular $\mu$ em $(G, \operatorname{Bor}(G))$ invariante por translações, chamada de medida de Haar. Um caso particular é da medida de Haar é a medida de Lebesgue em $\mathbb{R}^{n}$.

Por mais que um espaço de Banach de dimensão infinita não seja localmente compacto, podemos definir o que é um subconjunto Haar nulo de $X$ e iremos provar que para certos espaços de Banach $Y$, a menos de um conjunto Haar nulo de $X$, toda função $f: X \rightarrow Y$ Lipschitz será diferenciável com respeito a Gâteaux, onde este conceito de diferenciabilidade será definido nas seções seguintes. Ao longo do capítulo, todos espaços normados serão espaços em $\mathbb{R}$.

\subsection{Conjuntos Haar Nulos}

Os conjuntos Haar nulos vão traduzir o conceito de conjuntos com medida de Haar nula para um contexto mais geral de espaços e sem precisar definir uma medida.

Em 2.2.2, definimos vetor aleatório em espaços euclidianos. Essa definição se estende para os espaços de Banach.

Definição 3.1.1. Sejam $(\Omega, \mathcal{F}, \mathbb{P})$ um espaço de probabilidade e $X$ um espaço de Banach. Dizemos que uma função $\xi: \Omega \rightarrow X$ é um vetor aleatório se $\xi$ é uma função mensurável.

Definição 3.1.2. Sejam $X$ um espaço de Banach e $h: X \rightarrow[0, \infty]$ uma função Borelmensurável. Dizemos que $h$ é Haar nula se existem um espaço de probabilidade $(\Omega, \mathcal{F}, \mathbb{P})$ e um vetor aleatório $\xi: \Omega \rightarrow X$ tal que $\mathbb{E}(h(\xi+x))=0$ para cada $x \in X$. Em particular, dizemos que um boreliano $A \subset X$ é Haar nulo se a função $\chi_{A}$ é Haar nula.

Proposição 3.1.3. Sejam $X$ um espaço de Banach e $A \subset X$ boreliano. Então $A$ é Haar nulo se e somente se existe uma medida de probabilidade $\mu$ em $(X, B \operatorname{Bor}(X))$ satisfazendo $\mu(A+x)=0$ para cada $x \in X$.

Demonstração. Sejam $A$ um conjunto Haar nulo, $(\Omega, \mathcal{F}, \mathbb{P})$ um espaço de probabilidade e $\xi: \Omega \rightarrow X$ vetor aleatório satisfazendo a condição acima. Então $\int_{\Omega} \chi_{A}(\xi(\omega)+x) d \mathbb{P}=0$ para todo $x \in X$. Mas então, para todo $x \in X, \chi_{A}(\xi(\omega)+x)=0$ para quase todo $\omega \in \Omega$ com respeito a medida $\mathbb{P}$. Assim, para todo $x \in X$, temos $\xi(\omega) \notin A-x$ para quase todo $\omega$, que é equivalente a dizer que $\mathbb{P}\left(\xi^{-1}[A-x]\right)=0$. Tomamos então $\mu$ como sendo a medida distribuição de $\xi$.

Reciprocamente, tomamos $\xi=I d: X \rightarrow X$. Tomando $(\Omega, \mathcal{F}, \mathbb{P})=(X, \operatorname{Bor}(X), \mu)$, temos para cada $x \in X$ que

$$
\mathbb{E}\left(\chi_{A}(\xi+x)\right)=\int_{X} \chi_{A}(y+x) d \mu(y)=\int_{X} \chi_{A-x} y d \mu(y)=\mu(A-x)=0
$$

pois $\chi_{A}(y+x)=1$ se e somente se $y \in A-x$. 
Exemplo 3.1.4. Todo subconjunto boreliano $A$ de $\mathbb{R}^{n}$ com medida de Lebesgue nula é um conjunto Haar nulo. De fato, seja $\varphi: \mathbb{R}^{n} \rightarrow[0,1]$ uma função Lebesgue integrável tal que $\int_{\mathbb{R}^{n}} \varphi(x) d x=1$. Definindo

$$
\mu(E)=\int_{E} \varphi(x) d x \text { para cada E Lebesgue mensurável, }
$$

obtemos uma medida de probabilidade em $\left(\mathbb{R}^{n}, \operatorname{Bor}\left(\mathbb{R}^{n}\right)\right)$ tal que $\mu(A+x)=0$ para todo $x \in \mathbb{R}^{n}$.

Definição 3.1.5. Seja $X$ um espaço de Banach. Definimos o espaço das funções limitadas $C_{b}(X)$ como sendo o espaço de todas funções $f: X \rightarrow \mathbb{R}$ satisfazendo $\sup _{x \in X}|f(x)|<\infty$.

Proposição 3.1.6. A função $\|\cdot\|_{\infty}: C_{b}(X) \rightarrow[0, \infty)$ dada por $\|f\|_{\infty}=\sup _{x \in X}|f(x)|$ define uma norma completa em $C_{b}(X)$

Demonstração. Segue da definição e desigualdade triangular em $\mathbb{R}$ que $\|\cdot\|$ é uma norma em $C_{b}(X)$. Vamos mostrar que é completa. Seja $\left(f_{n}\right)_{n \in \mathbb{N}}$ uma sequência Cauchy em $C_{b}(X)$. Seja $\epsilon>0$ e $N$ tal que

$$
\sup _{x \in X}\left|f_{n}(x)-f_{m}(x)\right|=\left\|f_{n}-f_{m}\right\|_{\infty}<\epsilon \text { para cada } n, m \geq N \text {. }
$$

Mas então, para todo $x \in X$, a sequência $\left(f_{n}(x)\right)_{n \in \mathbb{N}}$ é Cauchy e portanto converge para um $f(x) \in \mathbb{R}$. Como $\epsilon$ independe de $x$ na equação acima, temos que para cada $x \in X$

$$
\left|f_{n}(x)-f_{m}(x)\right|<\epsilon \text { para cada } n, m \geq N
$$

e tomando o limite em $n$ obtemos para cada $x \in X$

$$
\left|f(x)-f_{m}(x)\right|<\epsilon \text { para cada } m \geq N
$$

e portanto

$$
\sup _{x \in X}\left|f(x)-f_{m}(x)\right| \leq \epsilon \text { para cada } m \geq N,
$$

provando tanto que $f \in C_{b}(X)$ (pois $f=f_{m}+f-f_{m}$ ) quanto que $\left\|f-f_{m}\right\|_{\infty} \leq \epsilon$ para todo $m \geq N$, ou seja, $f$ é o limite da sequência $\left(f_{n}\right)_{n \in \mathbb{N}}$ na norma $\|\cdot\|_{\infty}$.

Proposição 3.1.7. Sejam $(\Omega, \mathcal{F})$ um espaço mensurável e

$$
S=S(\Omega, \mathcal{F})=\{\mu: \mathcal{F} \rightarrow \mathbb{R}: \mu \text { é uma medida real satisfazendo }|\mu|(X)<\infty\}
$$

Então $S$ é um espaço vetorial real e a variação total $|\cdot|: S \rightarrow[0, \infty)$ (veja 2.1.23) define uma norma em $S$. 
Demonstração. Vamos mostrar que $S$ é um subespaço do espaço vetorial de todas medidas reais. Sejam $\mu, \nu \in S$ e $\alpha \in \mathbb{R}$. Veja que

$$
\begin{aligned}
|\alpha \mu|(X) & =\sup \left\{\sum_{k=1}^{\infty}\left|\alpha \mu\left(E_{k}\right)\right|: X=\bigcup_{k=0}^{\infty} E_{k} \text { e } E_{k} \cap E_{l}=\emptyset \text { para } k \neq l\right\} \\
& =\sup \left\{|\alpha| \sum_{k=1}^{\infty}\left|\mu\left(E_{k}\right)\right|: X=\bigcup_{k=0}^{\infty} E_{k} \text { e } E_{k} \cap E_{l}=\emptyset \text { para } k \neq l\right\} \\
& =|\alpha||\mu|(X)<\infty .
\end{aligned}
$$

Além disso, temos que

$$
\begin{aligned}
|\mu+\nu|(X) & =\sup \left\{\sum_{k=1}^{\infty}\left|\mu\left(E_{k}\right)+\nu\left(E_{k}\right)\right|: X=\bigcup_{k=0}^{\infty} E_{k} \text { e } E_{k} \cap E_{l}=\emptyset \text { para } k \neq l\right\} \\
& \leq \sup \left\{\sum_{k=1}^{\infty}\left|\mu\left(E_{k}\right)\right|+\sum_{k=1}^{\infty}\left|\nu\left(E_{k}\right)\right|: X=\bigcup_{k=0}^{\infty} E_{k} \text { e } E_{k} \cap E_{l}=\emptyset \text { para } k \neq l\right\} \\
& \leq|\mu|(X)+|\nu|(X)<\infty .
\end{aligned}
$$

Segue então tanto que $S$ é um espaço vetorial real quanto que $|\cdot|$ é uma norma em $S$.

Definição 3.1.8. Seja $X$ um espaço de Banach. Definimos $\operatorname{Pr}(X)$ como o conjunto de todas medidas de probabilidade em $(X, \operatorname{Bor}(X))$.

Observação 3.1.9. Veja que $\operatorname{Pr}(X) \neq \emptyset$ pois para cada $x \in X$, a medida de Dirac dada por

$$
\delta_{x}(A)=\left\{\begin{array}{ll}
0 & x \notin A \\
1 & x \in A
\end{array} \text { para cada } A \in \operatorname{Bor}(X),\right.
$$

é uma medida de probabilidade em $(X, \operatorname{Bor}(X))$.

A proposição acima nos diz que $\operatorname{Pr}(X)$ é um subconjunto do espaço normado $S(X, \operatorname{Bor}(X))$ com a norma da variação total. Mas podemos mergulhar $\operatorname{Pr}(X)$ no dual de $C_{b}(X)$.

Proposição 3.1.10. Existe um mergulho isométrico $T: \operatorname{Pr}(X) \rightarrow C_{b}(X)^{*}$.

Demonstração. Note que se $\mu$ é uma medida de probabilidade em $(X, \operatorname{Bor}(X))$, então a função $\varphi_{\mu}: C_{b}(X) \rightarrow \mathbb{R}$ dada por

$$
\varphi_{\mu}(f)=\int_{X} f d \mu
$$

define um funcional linear contínuo em $C_{b}(X)$. Além disso, a norma deste funcional é dada por

$$
\sup _{\|f\|_{\infty}=1}\left|\int_{X} f d \mu\right| \leq \sup _{\|f\|_{\infty}=1} \int_{X}\|f\|_{\infty} d \mu=\mu(X)=\|\mu\| .
$$


Além disso, tomando $f=1$, obtemos

$$
\sup _{\|f\|_{\infty}=1}\left|\int_{X} f d \mu\right| \geq \mu(X)=\|\mu\| .
$$

Então a função $T: \operatorname{Pr}(X) \rightarrow C_{b}(X)^{*}$ dada por $T(\mu)=\varphi_{\mu}$ é uma isometria entre $\operatorname{Pr}(X)$ e um subconjunto de $C_{b}(X)^{*}$.

Podemos então equipar $\operatorname{Pr}(X)$ com a topologia fraca estrela de $C_{b}(X)^{*}$, ou seja, $\mu_{n} \rightarrow \mu$ nesta topologia se e somente se $\int_{X} f d \mu_{n} \rightarrow \int_{X} f d \mu$ para toda $f \in C_{b}(X)$.

Definição 3.1.11. Sejam $\mu, \nu \in \operatorname{Pr}(X)$. Definimos a convolução como sendo a medida $\mu * \nu: \mathcal{F} \rightarrow[0, \infty)$ dada por

$$
\mu * \nu(E)=\int_{X} \mu(E-y) d \nu(y) \text { para cada } E \in \operatorname{Bor}(X) .
$$

Observação 3.1.12. Note que

$$
\mu * \nu(X)=\int_{X} \mu(X-y) d \nu(y)=\int_{X} 1 d \nu(y)=\nu(X)=1,
$$

logo a medida $\int_{X} \mu(E-y) d \nu(y)$ também é uma medida de probabilidade.

Definição 3.1.13. Sejam $X$ espaço de Banach e $A \subset X$. Definimos para cada $\epsilon>0$ o conjunto $A_{\epsilon} \subset X$ por

$$
A_{\epsilon}=\{y \in X: d(y, A) \leq \epsilon\}
$$

Observação 3.1.14. Veja que se $A \subset X$ e $y \in X$, então $(A+y)_{\epsilon}=A_{\epsilon}+y$ para todo $\epsilon>0$. De fato, temos que

$$
d(x-y, A)=\inf _{a \in A}\|a+y-x\|=\inf _{a+y \in A+y}\|a+y-x\|=d(x, A+y)
$$

logo, para todo $\epsilon>0$ temos $x \in(A+y)_{\epsilon}$ se e somente se $x-y \in A_{\epsilon}$.

A seguir vamos definir a métrica de Lévy-Prokhorov no espaço $\operatorname{Pr}(X)$.

Lema 3.1.15. Sejam $X$ um espaço de Banach e $A \subset X$ e $\epsilon>0$ Então:

1. $\rho(\mu, \nu)=\inf \left\{\epsilon>0: \mu(A) \leq \nu\left(A_{\epsilon}\right)+\epsilon\right.$ e $\nu(A) \leq \mu\left(A_{\epsilon}\right)+\epsilon$ para todo $\left.A \in \operatorname{Bor}(X)\right\}$ é uma métrica completa em $\operatorname{Pr}(X)$.

2. $\rho(\mu * \nu, \nu) \leq \rho\left(\mu, \delta_{0}\right)$ para cada $\mu, \nu \in \operatorname{Pr}(X)$, onde $\delta_{0}$ é a medida de Dírac em 0 .

Demonstração. 1) Ver [Pro56].

2) Seja $\epsilon>0$ tal que $\mu(A) \leq \delta_{0}\left(A_{\epsilon}\right)+\epsilon$ e $\delta_{0}(A) \leq \mu\left(A_{\epsilon}\right)+\epsilon$ para todo $A \in \operatorname{Bor}(X)$. Fixe 
$A \in \operatorname{Bor}(X)$ e veja que $\delta_{0}\left((A-y)_{\epsilon}\right)=1$ se e somente se $0 \in(A-y)_{\epsilon}=A_{\epsilon}-y$ e isto acontece se e somente se $y \in A_{\epsilon}$. Se $f(y)=\delta_{0}(A-y)_{\epsilon}$ para todo $y \in X$, então temos que $f=\chi_{A_{\epsilon}}$ e assim

$$
\mu * \nu(A)=\int_{X} \mu(A-y) d \nu(y) \leq \int_{X} \delta_{0}\left((A-y)_{\epsilon}\right) d \nu(y)+\epsilon=\int_{X} \chi_{A_{\epsilon}} d \nu(y)+\epsilon=\nu\left(A_{\epsilon}\right)+\epsilon .
$$

Por outro lado, como $(A-y)_{\epsilon}=A_{\epsilon}-y$ e como $\delta_{0}(A-y)=1$ se e somente se $y \in A$, temos

$$
\begin{aligned}
\mu * \nu\left(A_{\epsilon}\right)=\int_{X} \mu\left(A_{\epsilon}-y\right) d \nu(y) & =\int_{X} \mu\left((A-y)_{\epsilon}\right) d \nu(y) \\
& \geq \int_{X} \delta_{0}(A-y) d \nu(y)-\epsilon \\
& =\int_{X} \chi_{A} d \nu(y)-\epsilon \\
& =\nu(A)-\epsilon .
\end{aligned}
$$

Portanto, como $\epsilon$ foi escolhido arbitrariamente, segue que $\rho(\mu * \nu, \nu) \leq \rho\left(\mu, \delta_{0}\right)$.

Teorema 3.1.16. Sejam $X$ um espaço de Banach e $\left(A_{n}\right)_{n \in \mathbb{N}}$ uma sequência de borelianos de $X$ Haar nulos. Então $\bigcup_{n \in \mathbb{N}} A_{n}$ é Haar nulo.

Demonstração. Vamos começar demonstrando dois fatos.

Fato 1 : Se $A$ e $B$ são Haar nulos, então $A \cup B$ também é um conjunto Haar nulo.

Demonstração do Fato 1. De fato, sejam $\mu$ e $\nu$ as medidas dadas pela Proposição 3.1.3 para $A$ e $B$, respectivamente. Considere então a convolução $\mu * \nu$. Temos que para todo $x \in X$,

$$
\mu * \nu(A+x)=\int_{X} \mu(A+x-y) d \nu(y)=\int_{X} 0 d \nu(y)=0 .
$$

Também, como $x+y \in B$ se e somente se $y \in B-x$, temos

$$
\int_{X} \chi_{B}(x+y) d \nu(y)=\int_{X} \chi_{B-x}(y) d \nu(y)=\nu(B-x)=0,
$$

e analogamente $\mu(B-y)=\int_{X} \chi_{B}(x+y) d \mu(x)$. Segue então do Teorema de Fubini 2.1.18 que

$$
\begin{aligned}
\mu * \nu(B+x)=\int_{X} \mu(B-y) d \nu(y) & =\int_{X} \int_{X} \chi_{B}(x+y) d \mu(x) d \nu(y) \\
& =\int_{X \times X} \chi_{B}(x+y) d(\mu \otimes \nu)(x, y) \\
& =\int_{X} \int_{X} \chi_{B}(x+y) d \nu(y) d \mu(x) \\
& =\int_{X} \nu(B-x) d \mu(y)=0 .
\end{aligned}
$$


Então para todo $x \in X$, temos

$$
\mu * \nu(A \cup B+x)=\mu * \nu((A+x) \cup(B+x))=0
$$

Fato 2: Seja $A$ Haar nulo e $\mu$ a medida dada por 3.1.3. Para cada vizinhança aberta $U$ de $\operatorname{Pr}(X)$ contendo $\delta_{0}$, existe uma medida $\mu_{U} \in U$ tal que $\mu_{U}(A+x)=0$ para todo $x \in X$.

Demonstração do Fato 2. Vamos mostrar para as vizinhanças básicas $U=V\left(\delta_{0} ; f_{1}, \ldots, f_{n}, \epsilon\right) \cap$ $\operatorname{Pr}(X)$, onde $f_{1}, \ldots, f_{n} \in C_{b}(X)$. Tome $V$ um aberto de $X$ contendo 0 tal que $\left|f_{i}(0)-f_{i}(y)\right|<$ $\epsilon / 2$ para todo $y \in V$ e $i \in\{1, \ldots, n\}$. Defina $\mu_{U}(E)=\mu(E \cap V) / \mu(V)$ para todo $E \in \operatorname{Bor}(X)$.

Temos então

$$
\mu_{U}(A+x)=\mu((A+x) \cap V) / \mu(V)=0 \text { para todo } x \in X
$$

Além disso, para cada $i \in\{1, \ldots, n\}$ temos

$$
\begin{aligned}
\left|\delta_{0}\left(f_{i}\right)-\mu_{U}\left(f_{i}\right)\right|=\left|\int_{X} f_{i} d \delta_{0}-\int_{X} f_{i} d \mu_{U}\right| & =\left|\int_{X} f_{i} d \delta_{0}-\frac{1}{\mu(V)} \int_{V} f_{i} d \mu\right| \\
& =\left|f_{i}(0)-\frac{1}{\mu(V)} \int_{V} f_{i} d \mu\right| \\
& =\left|\frac{1}{\mu(V)} \int_{V}\left(f_{i}(0)-f_{i}(x)\right) d \mu(x)\right| \\
& \leq \frac{1}{\mu(V)} \int_{V}\left|f_{i}(0)-f_{i}(x)\right| d \mu(x) \\
& \leq \epsilon / 2<\epsilon .
\end{aligned}
$$

Logo $\mu_{U} \in U$ e o fato segue.

Em particular, tomando $\epsilon>0$ e o aberto $U=B\left(\delta_{0} ; \epsilon\right)$ em $\operatorname{Pr}(X)$, segue que para cada $A$ Haar nulo e $\epsilon>0$, existe $\mu_{U}$ tal que $\mu_{U}(A+x)=0$ para todo $x \in X$ e $\rho\left(\delta_{0}, \mu_{U}\right)<\epsilon$. Podemos então construir para todo $n \in \mathbb{N}$ uma medida $\mu_{n}$ tal que $\mu_{n}\left(A_{n}+x\right)=0$ para todo $x \in X$ e $\rho\left(\mu_{n}, \delta_{0}\right)<2^{-n}$. Logo

$$
\rho\left(\mu_{n} * \alpha, \alpha\right) \leq \rho\left(\mu_{n}, \delta_{0}\right)<2^{-n}
$$

onde $\alpha$ é qualquer convolução de medidas do conjunto $\left\{\mu_{k}: k=1, \ldots, n-1\right\}$ e $n \in \mathbb{N}$. Assim, a sequência dada por $\left(\prod_{k=1}^{N} * \mu_{k}\right)_{N \in \mathbb{N}}$ é Cauchy em $\operatorname{Pr}(X)$. Sendo $\operatorname{Pr}(X)$ um espaço métrico completo, está bem definido o produto $\mu=\prod_{k=1}^{\infty} * \mu_{k}=\lim _{N \in \mathbb{N}} \prod_{k=1}^{N} * \mu_{k}$. Veja que para cada $n \in \mathbb{N}$, temos $\mu=\mu_{n} * \nu_{n}$, onde $\nu_{n}=\prod_{k \neq n} * \mu_{k}$ e logo, pelo que mostramos na prova do Fato 1 , temos 
$\mu\left(A_{n}+x\right)=0$ para todo $n \in \mathbb{N}$ e para todo $x \in X$. Portanto $\mu\left(\bigcup_{n \in \mathbb{N}} A_{n}+x\right)=0$ para todo $x \in X$.

\subsection{Integral de Bochner}

Nesta seção vamos desenvolver o conceito de integração de Bochner. O processo para definir esta integral é muito análogo ao processo da integral de Lebesgue.

Vamos fixar um espaço de medida $\sigma$-finito $(\Omega, \mathcal{F}, \mu)$.

Definição 3.2.1. Uma função simples $s: \Omega \rightarrow X$ é mensurável se $s=\sum_{i=1}^{m} \chi_{A_{i}} x_{i}$, onde cada $A_{i}$ é mensurável. Neste caso, definimos ainda $\int_{A} s d \mu=\sum_{i=1}^{m} \mu\left(A_{i}\right) x_{i}$.

Observação 3.2.2. A definição da integral $\int_{A}$ sdu independe da representação de s.

Definição 3.2.3. Uma função $f: \Omega \rightarrow X$ é mensurável se existe uma sequência $\left(s_{n}\right)_{n \in \mathbb{N}}$ de funções simples mensuráveis em $(\Omega, \mathcal{F}, \mu)$ convergindo quase sempre para $f$.

Proposição 3.2.4. Uma função $f: \Omega \rightarrow X$ é mensurável se e somente se:

1. Para todo $x^{*} \in X^{*}$, a função $\omega \mapsto x^{*}(f(\omega))$ é mensurável.

2. Existe $A \in \mathcal{F}$ tal que $\mu(X \backslash A)=0 e[f(\omega): \omega \in A]$ é separável.

Demonstração. Seja $f: \Omega \rightarrow X$ mensurável. Seja $\left(s_{n}\right)_{n \in \mathbb{N}}$ uma sequência de funções simples mensuráveis convergindo quase sempre a $f$. Seja então $\left(x^{*} \circ s_{n}\right)_{n \in \mathbb{N}}$ são funções simples escalares convergindo quase sempre a $x^{*} \circ f$, logo esta é mensurável. Seja $Y$ o subespaço fechado de $X$ gerado por todos $s_{n}[\Omega]$. Veja que como cada $s_{n}[\Omega]$ tem dimensão finita, $Y$ é separável pois é gerado por um conjunto enumerável. Além disso, seja $A \in \mathcal{F}$ tal que $f(x)=\lim _{n \in \mathbb{N}} s_{n}(x)$ para todo $x \in A$ e tal que $\mu(X \backslash A)=0$. Então $f(x) \in Y$ para todo $x \in A$ e isto prova 2).

Para a volta, suponha sem perda de generalidade que existe um subespaço separável $Y$ de $X$ tal que $f(\omega) \in Y$ para todo $\omega \in \Omega$. Por 2.3.7, podemos encontrar $\left(y_{n}^{*}\right)_{n \in \mathbb{N}}$ em $S_{Y^{*}}$ tais que $\|y\|=\sup \left|y_{n}^{*}(y)\right|$ para todo $y \in Y$. Em particular, a função $\omega \mapsto\|f(\omega)-y\|$ será mensurável pois

$$
\|f(\omega)-y\|=\sup _{n}\left|y_{n}^{*}(f(\omega)-y)\right|
$$

isto é, a função é o supremo de funções que são mensuráveis por 1), e assim também é mensurável. Seja então $\epsilon>0,\left(y_{n}\right)_{n \in \mathbb{N}}$ densa em $Y$ e $A_{n}=\left\{\omega \in \Omega:\left\|f(\omega)-y_{n}\right\|<\epsilon\right\}$. Temos emtão $\Omega=\bigcup_{n \in \mathbb{N}} A_{n}$ e definindo $B_{n}=A_{n} \backslash \bigcup_{i=1}^{n-1} A_{i}$ e $g=\sum_{n \in \mathbb{N}} x_{n} \chi_{B_{n}}$, temos que $g$ é mensurável pois é limite de funções simples mensuráveis e $\|f(\omega)-g(\omega)\|<\epsilon$ por construção. Logo $f$ é mensurável. 
Definição 3.2.5. Dizemos que $f: \Omega \rightarrow X$ é Bochner integrável se existem $\left(s_{n}\right)_{n \in \mathbb{N}}$ simples mensuráveis convergindo quase sempre para $f$ tais que $\lim _{n, m} \int_{\Omega}\left\|s_{n}-s_{m}\right\| d \mu=0$, isto é, para todo $\epsilon>0$ existe $N \in \mathbb{N}$ tal que $n, m>N$ implica $\int_{\Omega}\left\|s_{n}-s_{m}\right\| d \mu<\epsilon$.

Definição 3.2.6. Dado um espaço de medida $(\Omega, \mathcal{F}, \mu)$ e um espaço de Banach $X$, definimos $L_{1}(\mu, X)$ como sendo o conjunto de todas funções $f: \Omega \rightarrow X$ Bochner integráveis.

Proposição 3.2.7. Sejam $f: \Omega \rightarrow X$ Bochner integrável e $\left(s_{n}\right)_{n \in \mathbb{N}}$ uma sequência de funções simples mensuráveis satisfazendo as condições da definição 3.2.5. Então

1. Existe $F: \Omega \rightarrow X$ mensurável tal que a função $\|F\|: \Omega \rightarrow[0, \infty)$ dada por $\|F\|(\omega)=$ $\|F(\omega)\|$ pertence a $L_{1}(\mu)$ e $\lim _{n \in \mathbb{N}} \int_{\Omega}\left\|F-s_{n}\right\| d \mu=0$.

2. $\lim _{n \in \mathbb{N}} \int_{\Omega} s_{n} d \mu$ existe e independe da escolha de $\left(s_{n}\right)_{n \in \mathbb{N}}$.

3. $\int_{\Omega}\|f\| d \mu$ é finito e $\lim _{n \in \mathbb{N}}\left\|\int_{\Omega} s_{n} d \mu\right\| \leq \int_{\Omega}\|f\| d \mu$.

Demonstração. 1) Seja $\left(s_{n_{i}}\right)_{i}$ uma subsequência de $\left(s_{n}\right)_{n \in \mathbb{N}}$ tal que $\int_{\Omega}\left\|s_{n_{i+1}}(\omega)-s_{n_{i}}(\omega)\right\| d \mu<$ $2^{-i}$. Definimos para cada $k \in \mathbb{N} g_{k}=\sum_{i=1}^{k}\left\|s_{n_{i+1}}-s_{n_{i}}\right\|$ e $g=\sum_{i=1}^{\infty}\left\|s_{n_{i+1}}-s_{n_{i}}\right\|$. Segue da desigualdade de Minkowski 2.1.11 que

$$
\left\|g_{k}\right\|_{L_{1}} \leq \sum_{i=1}^{k} \int_{\Omega}\left\|s_{n_{i+1}}(\omega)-s_{n_{i}}(\omega)\right\| d \mu<\sum_{i=1}^{k} 2^{-i}<1 \text { para cada } k \in \mathbb{N} .
$$

Segue então do Lema de Fatou 2.1.9 que

$$
\|g\|_{L_{1}}=\int_{\Omega}|g(\omega)| d \mu=\int_{\Omega} \liminf _{k}\left|g_{k}(\omega)\right| d \mu \leq \liminf _{k} \int_{\Omega}\left|g_{k}(\omega)\right| d \mu \leq 1
$$

Em particular, $g(x)<\infty$ quase sempre e logo a série $s_{n_{1}}+\sum_{i=1}^{\infty}\left(s_{n_{i+1}}-s_{n_{i}}\right)$ converge absolutamente para um $F(x)$ quase sempre. Para os pontos para os quais a série não convirja, deixemos $F(x)=0$. Como $s_{n_{k}}=s_{n_{1}}+\sum_{i=1}^{k}\left(s_{n_{i+1}}-s_{n_{i}}\right)$, segue que $F(x)=\lim _{i} s_{n_{i}}(x)$ quase sempre. Seja $\epsilon>0$. Existe $N$ tal que $\int_{\Omega}\left\|s_{n}-s_{m}\right\| d \mu<\epsilon$ para $n, m>N$. Para $m>N$ temos então, novamento pelo Lema de Fatou, que

$$
\int_{\Omega}\left|\|F\|-\left\|s_{m}\right\|\right| d \mu \leq \int_{\Omega}\left\|F-s_{m}\right\| d \mu \leq \liminf _{i} \int\left\|s_{n_{i}}-s_{m}\right\| d \mu \leq \epsilon
$$

Segue da desigualdade acima que $\lim _{m} \int_{\Omega}\left\|F-s_{m}\right\| d \mu=0$ e que $\|F\| \in L_{1}(\mu)$, pois $\|F\|-$ $\left\|s_{N+1}\right\| \in L_{1}(\mu)$ e $\left\|s_{N+1}\right\| \in L_{1}(\mu)$.

2) Pela desigualdade triangular temos

$$
\left\|\int_{\Omega} s_{n} d \mu-\int_{\Omega} s_{m} d \mu\right\| \leq \int_{\Omega}\left\|s_{n}-s_{m}\right\| d \mu
$$


Assim, decorre que $\left(\int_{\Omega} s_{n} d \mu\right)_{n \in \mathbb{N}}$ é uma sequência Cauchy em $X$ e logo convergente. Seja $F: \Omega \rightarrow X$ construída em 1). Em particular, mostramos que existe uma subsequência de $\left(s_{n}\right)_{n \in \mathbb{N}}$ convergindo quase sempre para $F$. Logo $F=f$ quase sempre e $\lim _{n \in \mathbb{N}} \int_{\Omega}\left\|f-s_{n}\right\| d \mu=0$. Assim, se $\left(r_{n}\right)_{n \in \mathbb{N}}$ é outra sequência de funções simples satisfazendo as mesmas propriedades, teremos que

$$
\begin{aligned}
\left\|\int_{\Omega} s_{n} d \mu-\int_{\Omega} r_{n} d \mu\right\| & \leq\left\|\int_{\Omega} s_{n} d \mu-\int_{\Omega} f d \mu\right\|+\left\|\int_{\Omega} f d \mu-\int_{\Omega} r_{n} d \mu\right\| \\
& \leq \int_{\Omega}\left\|f-s_{n}\right\| d \mu+\int_{\Omega}\left\|f-r_{n}\right\| d \mu
\end{aligned}
$$

e a última expressão tende a 0 pelo que provamos acima, mostrando que o limite independe da escolha das funções simples.

3) Como existe o limite $\lim _{n \in \mathbb{N}} \int_{\Omega}\left\|f-s_{n}\right\| d \mu$, existe $n$ suficientemente grande tal que $\int_{\Omega} \| f-$ $s_{n} \| d \mu<1$, e como

$$
\|f\| \leq\left\|f-s_{n}\right\|+\left\|s_{n}\right\|
$$

segue que

$$
\int_{\Omega}\|f\| d \mu \leq \int_{\Omega}\left\|f-s_{n}\right\| d \mu+\int_{\Omega}\left\|s_{n}\right\| d \mu<1+\int_{\Omega}\left\|s_{n}\right\| d \mu<\infty .
$$

Além disso,

$$
\begin{aligned}
\lim _{n \in \mathbb{N}}\left\|\int_{\Omega} s_{n} d \mu\right\| & =\liminf _{n \in \mathbb{N}}\left\|\int_{\Omega} s_{n} d \mu\right\| \\
& \leq \liminf _{n \in \mathbb{N}} \int_{\Omega}\left\|s_{n}\right\| d \mu \\
& \leq \liminf _{n \in \mathbb{N}} \int_{\Omega}\left(\left\|f-s_{n}\right\|\right) d \mu+\int_{\Omega}\|f\| d \mu \\
& =\int_{\Omega}\|f\| d \mu .
\end{aligned}
$$

Definição 3.2.8. Sejam $f: \Omega \rightarrow X$ uma função Bochner integrável e $\left(s_{n}\right)_{n \in \mathbb{N}}$ uma sequência de funções simples convergindo quase sempre para $f$ satisfazendo $\lim _{n, m} \int_{\Omega}\left\|s_{n}-s_{m}\right\| d \mu=0$. Definimos a integral de Bochner de $f$ como sendo $\lim _{n \in \mathbb{N}} \int_{\Omega} s_{n} d \mu$ e a denotamos por $\int_{\Omega} f d \mu$.

Proposição 3.2.9. Seja $f: \Omega \rightarrow X$. Então $f$ é Bochner integrável se e somente se $f$ é mensurável e $\int_{\Omega}\|f\| d \mu<\infty$.

Demonstração. A ida segue da Proposição 3.2.7. Para a volta, suponha também $\mu$ finita. Vimos na demonstração de 3.2.4 que para cada $\epsilon>0$ existe uma $g=\sum_{n \in \mathbb{N}} x_{n} \chi_{B_{n}}$ tal que 
$\|f(\omega)-g(\omega)\|<\epsilon$ para cada $\omega \in \Omega$. Então

$$
\begin{aligned}
\int_{\Omega}\left\|f(\omega)-\sum_{i=1}^{n} x_{i} \chi_{B_{i}}\right\| d \mu & =\int_{\bigcup_{i \leq n} B_{i}}\|f(\omega)-g(\omega)\| d \mu+\int_{\bigcup_{i>n} B_{i}}\|f(\omega)\| d \mu \\
& <\epsilon \mu(\Omega)+\int_{\bigcup_{i>n} B_{i}}\|f(\omega)\| d \mu .
\end{aligned}
$$

Como a medida $\mu$ é finita e a medida $E \mapsto \int_{E}\|f\| d \mu$ é absolutamente contínua com respeito a $\mu$, segue pelo Teorema 2.1.27 que o limite da expressão acima é 0 e portanto $f$ é Bochner integrável.

No caso em que $\Omega$ é a união disjunta $\bigcup_{n \in \mathbb{N}} A_{n} \operatorname{com} \mu\left(A_{n}\right)<\infty$ para todo $n \in \mathbb{N}$, então $f=\sum_{n \in \mathbb{N}} \chi_{A_{n}} f$ e cada $\chi_{A_{n}} f$ é Bochner integrável, logo $f$ é Bochner integrável.

Alguns teoremas clássicos de integração são válidos para a integral de Bochner.

Teorema 3.2.10. Sejam $f$ Bochner integrável e $f_{n}: \Omega \rightarrow X$ Bochner integrável para cada $n \in \mathbb{N}$.

1. (Teorema da convergência dominada) Se $f_{n} \rightarrow f$ quase sempre, $e\left\|f_{n}\right\| \leq g$ para alguma $g \in L_{1}(\Omega)$, então

$$
\lim _{n \in \mathbb{N}} \int_{\Omega}\left\|f-f_{n}\right\| d \mu=0
$$

2. Sejam $Y$ Banach e $T \in \mathcal{L}(X, Y)$. Então $T \circ f: \Omega \rightarrow Y$ é Bochner integrável e

$$
T\left(\int_{\Omega} f d \mu\right)=\int_{\Omega} T \circ f d \mu .
$$

3. Seja $f: \mathbb{R}^{m} \rightarrow X$ em $L_{1}\left(m_{\mathbb{R}^{m}}, X\right)$. Então vale para quase todo $u \in \mathbb{R}^{m}$ :

$$
\lim _{r \rightarrow 0^{+}} \frac{1}{m_{\mathbb{R}^{m}}(B(u ; r))} \int_{B(u ; r)}\|f(y)-f(u)\| d m_{\mathbb{R}^{m}}(y)=0 .
$$

4. Seja $f: \mathbb{R} \rightarrow X$ em $L_{1}(m, X)$ e $F: \mathbb{R} \rightarrow X$ dada por $F(x)=\int_{0}^{x} f(t) d t$. Então $F$ é diferenciável quase sempre e $F^{\prime}(x)=f(x)$.

Demonstração. 1) Note que $\|f\| \leq g$ e $\left\|f-f_{n}\right\| \leq 2 g$, o que nos dá $f, f-f_{n} \in L_{1}(\mu, X)$. Aplicando o Lema de Fatou para a função $2 g-\left\|f-f_{n}\right\|$ obtemos

$$
\begin{aligned}
\int_{\Omega} 2 g d \mu=\int_{\Omega}\left(2 g-\liminf _{n \in \mathbb{N}}\left\|f-f_{n}\right\|\right) d \mu & \leq \liminf _{n \in \mathbb{N}} \int_{\Omega}\left(2 g-\left\|f-f_{n}\right\|\right) d \mu \\
& =\int_{\Omega} 2 g d \mu+\liminf _{n \in \mathbb{N}}\left(-\int_{\Omega}\left\|f-f_{n}\right\| d \mu\right) \\
& =\int_{\Omega} 2 g d \mu-\limsup _{n \in \mathbb{N}} \int_{\Omega}\left\|f-f_{n}\right\| d \mu,
\end{aligned}
$$


assim

$$
\limsup _{n \in \mathbb{N}} \int_{\Omega}\left\|f-f_{n}\right\| d \mu \leq 0
$$

mas isto implica que

$$
\lim _{n \in \mathbb{N}} \int_{\Omega}\left\|f-f_{n}\right\| d \mu=0
$$

provando o que queríamos.

2) Se $\left(s_{n}\right)_{n \in \mathbb{N}}$ é uma sequência de funções simples convergindo a $f$ quase sempre, $\left(T \circ s_{n}\right)_{n \in \mathbb{N}}$ será uma sequência de funções simples convergindo a $T \circ f$ quase sempre, logo $T \circ f$ é mensurável. Além disso,

$$
\int_{\Omega}\|T \circ f\| d \mu \leq\|T\| \int_{\Omega}\|f\| d \mu
$$

que é finito pois $f$ é integrável. Logo $T \circ f$ é Bochner integrável pela Proposição 3.2.9. Por fim, se $f=\sum_{i=1}^{k} \chi_{A_{i}} x_{i}$, então

$$
T\left(\int_{\Omega} f d \mu\right)=\sum_{i=1}^{k} \mu\left(A_{i}\right) T\left(x_{i}\right)=\int_{\Omega} T \circ f d \mu .
$$

Em geral, basta tomar funções simples convergindo quase sempre a $f$.

3) Como a imagem de $f$ é um subconjunto separável de $X$, podemos supor sem perda de generalidade que $X$ é separável. Seja $\left(x_{n}\right)_{n \in \mathbb{N}}$ uma sequência densa em $X$. Aplicando o Teorema 2.1.14 para as funções $u \mapsto\left\|f(u)-x_{n}\right\|$, obtemos que

$$
\left\|f(u)-x_{n}\right\|=\lim _{r \rightarrow 0^{+}} \frac{1}{m_{\mathbb{R}^{m}}(B(u ; r))} \int_{B(u ; r)}\left\|f(y)-x_{n}\right\| d m_{\mathbb{R}^{m}}(y)
$$

para quase todo $u \in \mathbb{R}^{m}$, para todo $n \in \mathbb{N}$. Segue dai e da desigualdade triangular que para quase todo $u \in \mathbb{R}^{m}$, temos para todo $n \in \mathbb{N}$

$$
\limsup _{r \rightarrow 0^{+}} \frac{1}{m_{\mathbb{R}^{m}}(B(u ; r))} \int_{B(u ; r)}\|f(y)-f(u)\| d m_{\mathbb{R}^{m}}(y) \leq 2\left\|f(u)-x_{n}\right\| .
$$

Como $\left(x_{n}\right)_{n \in \mathbb{N}}$ é uma sequência densa, segue que o limite acima é 0, e o resultado segue.

4) Note que temos as seguintes desigualdades para todo $r>0$

$$
0 \leq \frac{1}{2 r} \int_{u-r}^{u}\|f(y)-f(u)\| d y \leq \frac{1}{2 r} \int_{u-r}^{u+r}\|f(y)-f(u)\| d y
$$

$\mathrm{e}$

$$
0 \leq \frac{1}{2 r} \int_{u}^{u+r}\|f(y)-f(u)\| d y \leq \frac{1}{2 r} \int_{u-r}^{u+r}\|f(y)-f(u)\| d y
$$


Por 3) obtemos que

$$
\lim _{r \rightarrow 0^{+}} \frac{1}{2 r} \int_{u-r}^{u+r}\|f(y)-f(u)\| d y=0
$$

$\log \mathrm{O}$

$$
\lim _{r \rightarrow 0^{+}} \frac{1}{r} \int_{u-r}^{u}\|f(y)-f(u)\| d y=0 \text { e } \lim _{r \rightarrow 0^{+}} \frac{1}{r} \int_{u}^{u+r}\|f(y)-f(u)\| d y=0
$$

Pela desigualdade triangular obtemos

$$
\lim _{r \rightarrow 0^{+}}\left\|\frac{1}{r} \int_{u-r}^{u} f(y) d y-f(u)\right\|=0 \text { e } \lim _{r \rightarrow 0^{+}}\left\|\frac{1}{r} \int_{u}^{u+r} f(y) d y-f(u)\right\|=0 .
$$

Mas

$$
\frac{1}{r}(F(u+r)-F(u))=-\frac{1}{r} \int_{u-(-r)}^{u} f(y) d y \text { para } r<0
$$

e

$$
\frac{1}{r}(F(u+r)-F(u))=\frac{1}{r} \int_{u}^{u+r} f(y) d y \text { para } r>0 .
$$

Portanto

$$
\lim _{r \rightarrow 0}\left\|\frac{1}{r}(F(u+r)-F(u))-f(u)\right\|=0 .
$$

Definição 3.2.11. Seja $f: \mathbb{R}^{n} \rightarrow X$ em $L_{1}\left(m_{\mathbb{R}^{n}}, X\right)$. Seja $u \in \mathbb{R}^{n}$ satisfazendo

$$
\lim _{r \rightarrow 0^{+}} \frac{1}{m_{\mathbb{R}^{n}}(B(u ; r))} \int_{B(u ; r)}\|f(y)-f(u)\| d m_{\mathbb{R}^{n}}(y)=0
$$

Dizemos então que u é um ponto de Lebesgue.

Enunciemos um resultado clássico na teoria de Análise Real.

Teorema 3.2.12 (Radon-Nikodým). Seja $(\Omega, \mathcal{F})$ um espaço mensurável. Sejam $\mu$ uma medida positiva $\sigma$-finita e $\nu$ uma medida complexa absolutamente continua com respeito a $\mu$. Então existe uma função $g: \Omega \rightarrow \mathbb{C} \mu$-integrável tal que

$$
\nu(A)=\int_{A} g d \mu \text { para cada } A \in \mathcal{F} .
$$

A seguir, vamos estudar os espaços para os quais vale este resultado (generalizando o conceito de medida em $\mathbb{C}$ para medidas em espaços de Banach $X$ ). Esta propriedade será fundamental para que possamos linearizar funções Lipschitz.

Definição 3.2.13. Sejam $(\Omega, \mathcal{F})$ um espaço mensurável e $X$ um espaço de Banach. Uma função $\tau: \mathcal{F} \rightarrow X$ é uma $X$-medida se $\tau(\emptyset)=0$ e $\tau\left(\bigcup_{n \in \mathbb{N}} E_{n}\right)=\sum_{n \in \mathbb{N}} \tau\left(E_{n}\right)$ sempre que $\left(E_{n}\right)_{n \in \mathbb{N}}$ for uma sequência de mensuráveis disjuntos.

Exemplo 3.2.14. Seja $f: \Omega \rightarrow X$ Bochner integrável. Então a função $\tau: \mathcal{F} \rightarrow X$ dada por $\tau(A)=\int_{A} f d \mu$ para cada $A \in \mathcal{F}$ define uma $X$-medida. 
Definição 3.2.15. Seja X um espaço de Banach. Diremos que X tem a propriedade de Radon-Nikodym (Ou simplesmente $X$ tem RNP) se satisfaz a seguinte propriedade:

Seja $(\Omega, \mathcal{F})$ um espaço de medida. Sejam $\tau$ uma $X$-medida e $\mu$ uma medida escalar de probabilidade em $(\Omega, \mathcal{F})$ tais que $\|\tau(A)\| \leq \mu(A)$ para todo $A \in \mathcal{F}$ com $\mu(A) \neq 0$. Então existe uma função $f \in L_{1}(\mu, X)$ tal que:

$$
\tau(A)=\int_{A} \text { fdu para todo } A \in \mathcal{F}
$$

\section{Teorema 3.2.16.}

1. Todo espaço de Banach reflexivo tem RNP.

2. Se $X=Z^{*}$ para algum espaço de Banach $Z$ e $X$ é separável, então $X$ tem $R N P$.

Demonstração. Ver [BL00] Corolário 5.12, página 107.

Exemplo 3.2.17. $c_{0}$ e $L_{1}$ não têm RNP. Veja [BL00] página 103 para demonstração.

\subsection{Teorema de Rademacher para espaços de Banach}

Nesta seção vamos finalmente demonstrar a generalização do teorema de Rademacher para funções $f: X \rightarrow Y$ entre um espaço de Banach $X$ e um espaço de Banach $Y$ com RNP. Vamos começar restringindo o domínio ao intervalo $[0,1]$, depois considerarmos espaços euclidianos até que finalmente vamos demonstrar para um espaço de Banach $X$ qualquer.

Definição 3.3.1. Sejam $X$ e $Y$ espaços de Banach e $f: X \rightarrow Y$ uma função. Dizemos que $f$ é Gâteaux diferenciável em $x_{0} \in X$ se existe $T \in \mathcal{L}(X, Y)$ tal que $\lim _{t \rightarrow 0} \frac{r(t x)}{t}=0$, onde

$$
r(x)=f\left(x_{0}+x\right)-f\left(x_{0}\right)-T(x) .
$$

Além disso, chamamos $T$ de derivada de Gâteaux de $f$ em $x_{0}$.

Em Análise, trabalha-se mais com o conceito de derivada de Fréchet, onde é necessário que o resto $r$ acima satisfaça $\lim _{\|x\| \rightarrow 0} \frac{r(x)}{\|x\|}=0$. Entretanto, a derivada de Gâteaux existe em condições mais gerais e será o suficiente para nossas aplicações.

Lema 3.3.2. Sejam $X$ e $Y$ espaços de Banach e $f: X \rightarrow Y$ uma função. Se $f$ é Gâteaux diferenciável em $x_{0} \in X$, então a derivada de Gâteaux de $f$ em $x_{0}$ é única.

Demonstração. Sejam $S$ e $R$ em $\mathcal{L}(X, Y)$ satisfazendo $\lim _{t \rightarrow 0} \frac{s(t x)}{t}=0$ e $\lim _{t \rightarrow 0} \frac{r(t x)}{t}=0$, onde

$$
s(x)=f\left(x+x_{0}\right)-f\left(x_{0}\right)-S(x) \text { e } r(x)=f\left(x+x_{0}\right)-f\left(x_{0}\right)-R(x) .
$$


Então para cada $x \in X$ e $t \in \mathbb{R} \backslash\{0\}$, temos

$$
S(x)-R(x)=\frac{S(t x)-R(t x)}{t}=\frac{r(t x)-s(t x)}{t}
$$

Portanto, para cada $x \in X$

$$
0=\lim _{t \rightarrow 0} \frac{r(t x)-s(t x)}{t}=S(x)-R(x)
$$

Ou seja, $S=R$ e o resultado segue.

Por conta deste resultado, podemos denotar a derivada de Gâteaux de $f$ em $x_{0}$ por $D_{f}\left(x_{0}\right)$.

Definição 3.3.3. Sejam $I \subset \mathbb{R}$ um intervalo e $f: I \rightarrow \mathbb{R}$ uma função. Dizemos que $f$ é absolutamente contínua se para todo $\epsilon>0$, existe um $\delta>0$ tal que para toda sequência de intervalos disjuntos $\left(\left(x_{k}, y_{k}\right)\right)_{k \in \mathbb{N}}$ satisfazendo $\left(x_{k}, y_{k}\right) \subset I$ para cada $k \in \mathbb{N} e$

$$
\sum_{k \in \mathbb{N}}\left(y_{k}-x_{k}\right)<\delta
$$

vale a desigualde

$$
\sum_{k \in \mathbb{N}}\left|f\left(y_{k}\right)-f\left(x_{k}\right)\right|<\delta
$$

O resultado a seguir é um resultado clássico de Análise Real.

Teorema 3.3.4. Sejam $I \subset \mathbb{R}$ um intervalo e $f: I \rightarrow \mathbb{R}$ absolutamente contínua. Então existe a derivada $f^{\prime}(x)$ para quase todo $x \in I$.

Demonstração. Ver Teorema [Nie97] 20.8.

Lema 3.3.5. Sejam $X$ espaço de Banach e $f:[0,1] \rightarrow X$ uma função lipschitziana. Então a função $F:[0,1] \rightarrow[0, \infty)$ dada por

$$
F(x)=\sup \left\{\sum_{i=1}^{m}\left\|f\left(a_{i}\right)-f\left(a_{i-1}\right)\right\|: 0=a_{0}<a_{1}<\ldots<a_{m}=x\right\} \text { para cada } x \in[0,1] .
$$

é uma função crescente e lipschitziana.

Demonstração. Sejam $0 \leq x<y \leq 1$. Sejam $a_{0}, \ldots, a_{m} \in[0,1]$ tais que $0=a_{0}<a_{1}<\ldots<$ $a_{m}=x$. Seja $a_{m+1}=y$. Então

$$
\sum_{i=1}^{m}\left\|f\left(a_{i}\right)-f\left(a_{i-1}\right)\right\| \leq \sum_{i=1}^{m+1}\left\|f\left(a_{i}\right)-f\left(a_{i-1}\right)\right\| \leq F(y)
$$


Pela arbitrariedade da partição, segue que $F(x) \leq F(y)$.

Afirmamos agora que

$$
F(y)=F(x)+\sup \left\{\sum_{i=1}^{m}\left\|f\left(a_{i}\right)-f\left(a_{i-1}\right)\right\|: x=a_{0}<a_{1}<\ldots<a_{m}=y\right\} .
$$

De fato, sejam $b_{0}, \ldots, b_{m} \in[0,1]$ tais que $0=b_{0}<b_{1}<\ldots<b_{n}=y$. Seja $j \in\{0, \ldots, n\}$ o menor número tal que $x<b_{j}$. Então

$$
\begin{aligned}
\sum_{i=1}^{n}\left\|f\left(b_{i}\right)-f\left(b_{i-1}\right)\right\| & =\sum_{i=1}^{j-1}\left\|f\left(b_{i}\right)-f\left(b_{i-1}\right)\right\|+\sum_{i=j}^{n}\left\|f\left(b_{i}\right)-f\left(b_{i-1}\right)\right\| \\
& \leq \sum_{i=1}^{j-1}\left\|f\left(b_{i}\right)-f\left(b_{i-1}\right)\right\|+\left\|f(x)-f\left(b_{j-1}\right)\right\| \\
& +\left\|f\left(b_{j}\right)-f(x)\right\|+\sum_{i=j}^{n}\left\|f\left(b_{i}\right)-f\left(b_{i-1}\right)\right\| \\
& \leq F(x)+\sup \left\{\sum_{i=1}^{m}\left\|f\left(a_{i}\right)-f\left(a_{i-1}\right)\right\|: x=a_{0}<a_{1}<\ldots<a_{m}=y\right\} .
\end{aligned}
$$

E segue do fato da partição ter sido escolhida arbitrariamente que

$$
F(y) \leq F(x)+\sup \left\{\sum_{i=1}^{m}\left\|f\left(a_{i}\right)-f\left(a_{i-1}\right)\right\|: x=a_{0}<a_{1}<\ldots<a_{m}=y\right\} .
$$

Por outro lado, sejam $x=b_{0}<b_{1}<\ldots<b_{m}=y$ e $\delta>0$. Seja $0=a_{0}<a_{1}<\ldots<a_{n}=x$ tal que

$$
\sum_{i=1}^{m}\left\|f\left(a_{i}\right)-f\left(a_{i-1}\right)\right\|>F(x)-\delta
$$

Temos então que

$$
\begin{aligned}
\sum_{i=1}^{m}\left\|f\left(b_{i}\right)-f\left(b_{i-1}\right)\right\| & =\sum_{i=1}^{m}\left\|f\left(b_{i}\right)-f\left(b_{i-1}\right)\right\|+\sum_{i=1}^{n}\left\|f\left(a_{i}\right)-f\left(a_{i-1}\right)\right\|-\sum_{i=1}^{n}\left\|f\left(a_{i}\right)-f\left(a_{i-1}\right)\right\| \\
& \leq F(y)-F(x)+\delta
\end{aligned}
$$

e como $\delta$ é arbitrário, segue que

$$
\sum_{i=1}^{m}\left\|f\left(b_{i}\right)-f\left(b_{i-1}\right)\right\| \leq F(y)-F(x)
$$

e como a partição é arbitrária, a afirmação segue. 
Mas então,

$$
\begin{aligned}
0 \leq F(y)-F(x) & =\sup \left\{\sum_{i=1}^{m}\left\|f\left(a_{i}\right)-f\left(a_{i-1}\right)\right\|: x=a_{0}<a_{1}<\ldots<a_{m}=y\right\} \\
& \leq \operatorname{Lip}(f) \sup \left\{\sum_{i=1}^{m}\left(a_{i}-a_{i-1}\right): x=a_{0}<a_{1}<\ldots<a_{m}=y\right\} \\
& =\operatorname{Lip}(f)(y-x) .
\end{aligned}
$$

Teorema 3.3.6. Seja X um espaço de Banach com RNP. Então toda função $f:[0,1] \rightarrow X$ Lipschitz é diferenciável quase sempre.

Demonstração. Seja $F:[0,1] \rightarrow[0, \infty)$ como no Lema 3.3.5. Então $F$ é lipschitziana e pelo resultado 3.3.4 e pelo Teorema Fundamental do Cálculo, segue que $F(x)=\int_{0}^{x} F^{\prime}(t) d t+F(0)$ para quase todo $x \in[0,1]$. Definindo para cada $A \in \operatorname{Bor}([0,1])$

$$
\mu(A)=\int_{A} F^{\prime}(t) d t
$$

obtemos uma medida absolutamente contínua com respeito a medida de Lebesgue $m$ e que satisfaz $F(x)=\mu([0, x])$ para quase todo $x$. Para cada aberto $V \subset[0,1]$, podemos escrevê-lo como a união disjunta $\bigcup_{i=1}^{\infty}\left(a_{i-1}, a_{i}\right)$ e definimos

$$
\tau(V)=\sum_{i=1}^{\infty}\left(f\left(a_{i}\right)-f\left(a_{i-1}\right)\right)
$$

Afirmamos que $\|\tau(V)\| \leq \mu(V)$ para cada aberto $V$. De fato, vamos escrever $V$ como a união disjunta $\bigcup_{i=1}^{\infty}\left(a_{i-1}, a_{i}\right)$. Seja $N \in \mathbb{N}$. Então, pelo que provamos no Lema 3.3.5

$$
\begin{aligned}
\left\|\sum_{i=1}^{N}\left(f\left(a_{i}\right)-f\left(a_{i-1}\right)\right)\right\| & \leq \sum_{i=1}^{N}\left\|\left(f\left(a_{i}\right)-f\left(a_{i-1}\right)\right)\right\| \\
& \leq \sum_{i=1}^{N}\left(F\left(a_{i}\right)-F\left(a_{i-1}\right)\right) \\
& =\sum_{i=1}^{N} \int_{a_{i-1}}^{a_{i}} F^{\prime}(t) d t \\
& =\mu\left(\bigcup_{i=1}^{N}\left(a_{i-1}, a_{i}\right)\right) .
\end{aligned}
$$

Passando o limite em $N$, a afirmação segue.

Como a medida de Lebesgue $m$ é regular, para cada Boreliano $A \subset[0,1]$ existe um sequência decrescente de abertos $\left(V_{n}\right)_{n \in \mathbb{N}}$ contendo $A$ satisfazendo $\lim _{n \in \mathbb{N}} m\left(V_{n}\right)=m(A)$. Mas então 
$\lim _{n \in \mathbb{N}} m\left(V_{n} \backslash A\right)=0$. Como temos $\mu \prec m$ (ver Definição 2.1.25), segue que $\lim _{n \in \mathbb{N}} \mu\left(V_{n} \backslash A\right)=0$ e assim $\lim _{n \in \mathbb{N}} \mu\left(V_{n}\right)=\mu(A)$. Segue então que $\left(\tau\left(V_{n}\right)\right)_{n \in \mathbb{N}}$ é uma sequência de Cauchy, portanto existe $\tau(A)=\lim _{n \in \mathbb{N}} \tau\left(V_{n}\right)$.

Então $\tau$ é uma $X$-medida e $\|\tau(A)\| \leq \mu(A)$ para todo $A \in \operatorname{Bor}([0,1])$. De fato, se $\left(V_{n}\right)_{n \in \mathbb{N}}$ é uma sequência de abertos contendo $A$ e satisfazendo $\lim _{n \in \mathbb{N}} \mu\left(V_{n}\right)=\mu(A)$, então

$$
\|\tau(A)\|=\lim _{n \in \mathbb{N}}\left\|\tau\left(V_{n}\right)\right\| \leq \lim _{n \in \mathbb{N}}\left\|\mu\left(V_{n}\right)\right\|=\mu(A) .
$$

Portanto, como $X$ tem RNP e $\mu \prec m$, existe $g:[0,1] \rightarrow X$ em $L_{1}(m, X)$ tal que

$$
f(x)=\tau([0, x])+f(0)=\int_{0}^{x} g(t) d t+f(0)
$$

quase sempre e assim $g=f^{\prime}$ quase sempre por 3.2.10 4).

Lema 3.3.7. Sejam $X$ e $Y$ espaços de Banach, $x_{0} \in X$ e $f: X \rightarrow Y$ lipschitziana. Seja $G$ um subgrupo denso (aditivo) de $X$ tal que existam as derivadas direcionais de $f$ em $x_{0}$ nas direções dos elementos de $G$ e que a função $u \mapsto \lim _{t \rightarrow 0}(f(x+t u)-f(x)) / t$ seja aditiva em $u \in G$. Então $f$ é Gâteaux diferenciável em $x_{0}$.

Demonstração. Para cada $t \neq 0$ definimos $h_{t}: X \rightarrow Y$ por

$$
h_{t}(u)=\left(f\left(x_{0}+t u\right)-f\left(x_{0}\right)\right) / t \text { para cada } u \in X
$$

e também para cada $u \in G$, definimos

$$
T(u)=\lim _{t \rightarrow 0}\left(f\left(x_{0}+t u\right)-f\left(x_{0}\right)\right) / t=\lim _{t \rightarrow 0} h_{t}(u) .
$$

Note que $\left\|h_{t}(u)-h_{t}(v)\right\| \leq \operatorname{Lip}(f)\|u-v\|$ para cada $t \neq 0$. Temos então que

$$
\|T(u)-T(v)\| \leq \operatorname{Lip}(f)\|u-v\|
$$

para cada $u, v \in G$. Veja que se $\left(v_{n}\right)_{n \in \mathbb{N}}$ é uma sequência em $G$ convergente, então a sequência $\left(T\left(v_{n}\right)\right)_{n \in \mathbb{N}}$ é Cauchy e assim existe o limite $\lim _{n \in \mathbb{N}} T\left(v_{n}\right)_{n \in \mathbb{N}}$.

Se $v \in X$, definimos então $T(v)=\lim _{n \in \mathbb{N}} T\left(v_{n}\right)$, onde $v_{n} \rightarrow v$ e $v_{n} \in G$ para cada $n \in \mathbb{N}$. Veja que a definição de $T(v)$ independe da sequência $\left(v_{n}\right)_{n \in \mathbb{N}}$ escolhida em $G$ pois se $\left(v_{n}^{(1)}\right)_{n \in \mathbb{N}}$ e $\left(v_{n}^{(2)}\right)_{n \in \mathbb{N}}$ são sequências em $G$ convergentes a $v$, então

$$
\left\|T\left(v_{n}^{(1)}\right)-T\left(v_{n}^{(2)}\right)\right\| \leq \operatorname{Lip}(f)\left\|v_{n}^{(1)}-v_{n}^{(2)}\right\| \rightarrow 0 .
$$

Nosso objetivo é mostrar que $T=D_{f}\left(x_{0}\right)$. Vamos provar primeiro que $T(v)=\lim _{t \rightarrow 0}\left(f\left(x_{0}+\right.\right.$ $\left.t v)-f\left(x_{0}\right)\right) / t$. De fato, sejam $v \in X,\left(v_{n}\right)_{n \in \mathbb{N}}$ sequência em $G$ convergente a $v$ e seja $\epsilon>0$. 
Tome $n_{0}$ grande o suficiente de modo que

$$
\left\|v-v_{n_{0}}\right\|<\operatorname{Lip}(f)^{-1} \epsilon / 3 \text { e }\left\|T\left(v_{n_{0}}\right)-T(v)\right\|<\epsilon / 3
$$

Seja $\delta>0$ tal que $|t|<\delta$ implica $\left\|T\left(v_{n_{0}}\right)-h_{t}\left(v_{n_{0}}\right)\right\|<\epsilon / 3$. Então

$$
\begin{aligned}
\left\|h_{t}(v)-T(v)\right\| & \leq\left\|h_{t}(v)-h_{t}\left(v_{n_{0}}\right)\right\|+\left\|h_{t}\left(v_{n_{0}}\right)-T\left(v_{n_{0}}\right)\right\|+\left\|T\left(v_{n_{0}}\right)-T(v)\right\| \\
& \leq \operatorname{Lip}(f)\left\|v-v_{n}\right\|+2 \epsilon / 3 \\
& <\epsilon,
\end{aligned}
$$

provando o que queríamos. Para finalizar a demonstração basta provar que $T$ é linear e contínua. Sejam $u, v \in X$ e $\left(u_{n}\right)_{n \in \mathbb{N}}$ e $\left(v_{n}\right)_{n \in \mathbb{N}}$ sequências em $G$ convergindo a $u$ e $v$, respectivamente. Então

$$
\|T(u)-T(v)\|=\lim _{n \in \mathbb{N}}\left\|T\left(u_{n}\right)-T\left(v_{n}\right)\right\| \leq \lim _{n \in \mathbb{N}} \operatorname{Lip}(f)\left\|u_{n}-v_{n}\right\|=\operatorname{Lip}(f)\|u-v\|,
$$

Assim $T$ é uma função Lipschitz. Além disso segue da aditividade da hipótese que

$$
T(u+v)=\lim _{n \in \mathbb{N}} T\left(u_{n}+v_{n}\right)=\lim _{n \in \mathbb{N}} T\left(u_{n}\right)+\lim _{n \in \mathbb{N}} T\left(v_{n}\right)=T(u)+T(v)
$$

Resta provar que $T(\alpha u)=\alpha T(u)$ para cada $\alpha \in \mathbb{R}$. De fato, segue da aditividade que $T(n v)=n T(v)$ para todo $n \in \mathbb{Z}$ e $v \in X$. Além disso,

$$
T(v)=T\left(n \frac{1}{n} v\right)=n T\left(\frac{1}{n} v\right)
$$

$\operatorname{logo} T\left(\frac{1}{n} v\right)=\frac{1}{n} T(v)$ e $T(q v)=q T(v)$ para todo $q \in \mathbb{Q}$ e $v \in X$. Assim, se $\alpha \in \mathbb{R}$ e $\left(q_{n}\right)_{n \in \mathbb{N}}$ é uma sequência de racionais convergindo a $\alpha$, temos que

$$
T(\alpha v)=\lim _{n \in \mathbb{N}} T\left(q_{n} v\right)=\lim _{n \in \mathbb{N}} q_{n} T(v)=\alpha T(v)
$$

Definição 3.3.8. Dizemos que uma função $\varphi: \mathbb{R}^{n} \rightarrow \mathbb{R}$ tem suporte compacto se $\overline{\left\{x \in \mathbb{R}^{n}: \varphi(x) \neq 0\right\}}$ é um compacto de $\mathbb{R}^{n}$.

Observação 3.3.9. Note que se $\varphi: \mathbb{R}^{n} \rightarrow \mathbb{R}$ é uma função contínua com suporte compacto, e se $K=\overline{\left\{x \in \mathbb{R}^{n}: \varphi(x) \neq 0\right\}}$, então

$$
\int_{\mathbb{R}^{n}}|\varphi(x)| d x=\int_{K}|\varphi(x)| d x<\infty .
$$

Lema 3.3.10. Sejam $f: \mathbb{R}^{n} \rightarrow X$ lipschitziana $e \varphi: \mathbb{R}^{n} \rightarrow \mathbb{R}$ uma função $C^{1}$ com suporte 
compacto. Definindo a convolução por

$$
g(x)=f * \varphi(x)=\int_{\mathbb{R}^{n}} f(y) \varphi(x-y) d y \text { para cada } x \in \mathbb{R}^{n} .
$$

Então temos que

1. A convolução é comutativa, isto é,

$$
g(x)=\int_{\mathbb{R}^{n}} f(x-y) \varphi(y) d y \text { para cada } x \in \mathbb{R}^{n} .
$$

2. g é uma função diferenciável e

$$
D_{g}(x)(u)=\int_{\mathbb{R}^{n}}\left(f(y) D_{\varphi}(x-y)(u)\right) d y \text { para cada } x, u \in \mathbb{R}^{n} .
$$

Demonstração. 1) Sejam $x^{*} \in X^{*}$ e $G: \mathbb{R}^{n} \rightarrow \mathbb{R}^{n}$ dada por $G(y)=x-y$. Defina $F: \mathbb{R}^{n} \rightarrow \mathbb{R}$ por $F(y)=x^{*}(f(x-y) \varphi(y))$. Pelo Teorema de mudança de variáveis 2.1.21 temos que

$$
\int_{\mathbb{R}^{n}} x^{*}(f(x-y) \varphi(y)) d y=\int_{\mathbb{R}^{n}} F(y) d y=\int_{\mathbb{R}^{n}} F \circ G(y) d y=\int_{\mathbb{R}^{n}} x^{*}(f(y) \varphi(x-y)) d y .
$$

Mas pelo Teorema 3.2.10 2) obtemos que

$$
x^{*}\left(\int_{\mathbb{R}^{n}} f(x-y) \varphi(y) d y\right)=x^{*}\left(\int_{\mathbb{R}^{n}} f(y) \varphi(x-y) d y\right) .
$$

Pela arbitrariedade de $x^{*} \in X^{*}$ e por Hahn Banach segue o resultado.

2) Seja $K$ o suporte da função $\varphi$. Veja que $D_{\varphi}(x)$ é o operador nulo para todo $x \in K^{c}$. De fato, seja $U$ aberto contendo $x$ e contido em $K^{c}$. Então para todo $u \in \mathbb{R}^{n}$ e $t$ suficientemente pequeno, $\varphi(x+t u)-\varphi(x)=0$, $\log 0 D_{\varphi}(x)(u)=0$ para todo $u \in \mathbb{R}^{n}$. Vamos definir para cada $x \in \mathbb{R}^{n}$ o operador $T=T_{x}: \mathbb{R}^{n} \rightarrow X$ por

$$
T(u)=\int_{\mathbb{R}^{n}}\left(f(y) D_{\varphi}(x-y)(u)\right) d y=\int_{-K+x} f(y) D_{\varphi}(x-y)(u) d y .
$$

Como $\varphi$ é uma função $C^{1}$ temos então que $\sup _{y \in-K+x}\left\|D_{\varphi}(x-y)\right\|<\infty$ e

$$
\|T(u)\| \leq \int_{-K+x}\left\|\left(f(y) D_{\varphi}(x-y)(u)\right)\right\| d y \leq\left(\int_{-K+x}\left\|D_{\varphi}(x-y)\right\|\|f(y)\| d y\right)\|u\| .
$$

Logo $T$ é um operador contínuo. Vamos mostrar que $T=D_{g}(x)$. 
Definimos um compacto

$$
K^{\prime}=\bigcup_{-1 \leq t \leq 1}(-K+t u+x)=-K+[-1,1] u+x
$$

e então fazemos $C=(-K+x) \cup K^{\prime}$. Então $C$ é um compacto e além disso, para cada $y \in C^{c}$, temos que

$$
\varphi(x-y+t u)-\varphi(x-y)=0-0=0 \text { para todo }-1 \leq t \leq 1,
$$

pois $\varphi(x-y)=0$ para todo $y \in(-K+x)^{c}$ e $\varphi(x-y+t u)=0$ para todo $y \in\left(K^{\prime}\right)^{c} \mathrm{e}$ $t \in[-1,1]$.

Como

$$
\lim _{t \rightarrow 0}(f(y)[\varphi(x-y+t u)-\varphi(x-y)] / t)=\left(f(y) D_{\varphi}(x-y)(u)\right) \text { para cada } y \in C,
$$

então para cada $y \in C$ e $t \in[-1,1]$ suficientemente pequeno, temos

$$
\|f(y)[\varphi(x-y+t u)-\varphi(x-y)] / t\| \leq\left\|f(y) D_{\varphi}(x-y)(u)\right\|+\|f(y)\|,
$$

mas como

$$
\int_{C}\left(\left\|f(y) D_{\varphi}(x-y)(u)\right\|+\|f(y)\|\right) d y<\infty
$$

podemos aplicar o Teorema de convergência dominada 3.2.10 1) para concluir que

$$
\begin{aligned}
\lim _{t \rightarrow 0} \int_{\mathbb{R}^{n}} f(y)[\varphi(x-y+t u)-\varphi(x-y)] / t d y & =\lim _{t \rightarrow 0} \int_{C} f(y)[\varphi(x-y+t u)-\varphi(x-y)] / t d y \\
& =\int_{C} \lim _{t \rightarrow 0}(f(y)[\varphi(x-y+t u)-\varphi(x-y)] / t) d y \\
& =\int_{C} f(y) D_{\varphi}(x-y)(u) d y \\
& =\int_{-K+x} f(y) D_{\varphi}(x-y)(u) d y \\
& =\int_{\mathbb{R}^{n}} f(y) D_{\varphi}(x-y)(u) d y \\
& =T_{x}(u) .
\end{aligned}
$$

Teorema 3.3.11. Sejam $X$ um espaço de Banach com $R N P$ e $f: \mathbb{R}^{n} \rightarrow X$ uma função lipschitziana. Então f é diferenciável quase sempre em $\mathbb{R}^{n}$.

Antes de demonstrar este teorema, demonstremos o lema a seguir.

Lema 3.3.12. Sejam $X$ um espaço de Banach com $R N P$ e $f: \mathbb{R}^{n} \rightarrow X$ uma função lipschitziana. Então para cada $v \in \mathbb{R}^{n}$ o conjunto $S_{v}$ de todos os pontos $x \in \mathbb{R}^{n}$ para os quais 
existe a derivada direcional $\lim _{t \rightarrow 0}(f(x+t v)-f(x)) / t$ é Borel mensurável e $S_{v}^{c}$ tem medida de Lebesgue nula.

Demonstração do Lema. Afirmamos que a derivada direcional $\lim _{t \rightarrow 0}(f(x+t v)-f(x)) / t$ existe se e somente se existe o limite $\lim _{k \rightarrow \infty} k\left(f\left(x+\frac{1}{k} v\right)-f(x)\right)$. De fato, suponha que exista o limite sequencial acima, seja $k \in \mathbb{N}$ e tome $1 /(k+1) \leq t<1 / k$. Temos:

$$
\begin{aligned}
\left\|\frac{f(x+t v)-f(x)}{t}-k\left(f\left(x+\frac{1}{k} v\right)-f(x)\right)\right\| & = \\
\left\|\frac{f(x+t v)-f\left(x+\frac{1}{k} v\right)}{t}+(1 / t-k)\left(f\left(x+\frac{1}{k} v\right)-f(x)\right)\right\| & \leq \\
\frac{|t-1 / k| \operatorname{Lip}(f)\|v\|}{t}+\frac{(1 / t-k) \operatorname{Lip}(f)\|v\|}{k} & = \\
\frac{2 \operatorname{Lip}(f)\|v\|(1-k t)}{k t} & < \\
\frac{2 \operatorname{Lip}(f)\|v\|}{k} . &
\end{aligned}
$$

Segue então que

$$
\lim _{t \rightarrow 0} \frac{f(x+t v)-f(x)}{t}=\lim _{k \rightarrow \infty} k\left(f\left(x+\frac{1}{k} v\right)-f(x)\right)
$$

Assim, $S_{v}$ é o conjunto dos pontos para os quais existe o limite pontual da sequência de funções contínuas $\left(f_{k}\right)_{k}$, onde $f_{k}(x)=k\left(f\left(x+\frac{1}{k} v\right)-f(x)\right)$ e portanto é um conjunto Borel mensurável por 2.1.8.

Seja $R_{v}=S_{v}^{c}$. Vamos mostrar que $m_{\mathbb{R}^{n}}\left(R_{v}\right)=0$. Para $v, x \in \mathbb{R}^{n}$ fixos, definimos a função $g_{x, v}: \mathbb{R} \rightarrow X$ por $g_{x, v}(t)=f(x+t v)$. Note que $x+t_{0} v \in R_{v}$ se e somente se não existe a derivada em $t_{0}$ da função $g_{x, v}$. Seja $J: \mathbb{R}^{n} \rightarrow \mathbb{R}^{n}$ um isomorfismo linear com $J\left(e_{1}\right)=v$, onde $e_{1}=(1,0, \ldots, 0)$. Então, aplicando o Teorema da mudança de variáveis 2.1 .21 e o Teorema de Fubini 2.1.20, obtemos

$$
\begin{aligned}
m_{\mathbb{R}^{n}}\left(R_{v}\right)=\int_{\mathbb{R}^{n}} \chi_{R_{v}}(x) d m_{\mathbb{R}^{n}}(x) & =\int_{\mathbb{R}^{n}} \chi_{R_{v}} \circ J(x)|\operatorname{det}(d J(x))| d m_{\mathbb{R}^{n}}(x) \\
& =\alpha \int_{\mathbb{R}^{n}} \chi_{R_{v}} \circ J(x) d m_{\mathbb{R}^{n}}(x) \\
& =\alpha \int_{\mathbb{R}} \ldots \int_{\mathbb{R}} \chi_{R_{v}}\left(J\left(x_{1}, x_{2}, \ldots, x_{n}\right)\right) d m_{\mathbb{R}}\left(x_{1}\right) d m_{\mathbb{R}}\left(x_{2}\right) \ldots d m_{\mathbb{R}}\left(x_{n}\right),
\end{aligned}
$$

onde $\alpha=|\operatorname{det} J|$. Mas fixados $x_{2}, \ldots, x_{n}$, podemos aplicar o Teorema 3.3 .6 à função $g_{x, v}$, onde $x=J\left(0, x_{2}, \ldots, x_{n}\right)$ e assim

$$
\int_{\mathbb{R}} \chi_{R_{v}}\left(J\left(x_{1}, x_{2}, \ldots, x_{n}\right)\right) d m_{\mathbb{R}}\left(x_{1}\right)=\int_{\mathbb{R}} \chi_{R_{v}}\left(J\left(0, x_{2}, \ldots, x_{n}\right)+x_{1} v\right) d m_{\mathbb{R}}\left(x_{1}\right)=0 .
$$


Demonstração do Teorema. Seja $G$ um subgrupo aditivo de $\mathbb{R}^{n}$ que seja denso e enumerável. Pelo Lema 3.3.12, para quase todo $x \in \mathbb{R}^{n}$ existem as derivadas direcionais nas direções dos elementos de $G$. Vamos mostrar que as derivadas direcionais $h_{u}(x)=\lim _{t \rightarrow 0}(f(x+t u)-f(x)) / t$ são aditivas em $u$ para quase todo $x \in \mathbb{R}^{n}$ e assim o resultado seguirá do Lema 3.3.7. Seja $\varphi: \mathbb{R}^{n} \rightarrow \mathbb{R}$ qualquer função $C^{1}$ com suporte compacto e satisfazendo $\int_{\mathbb{R}^{n}} \varphi(x) d x=1$. Defina então $g=f * \varphi$ como no Lema 3.3.10. Vimos que $g$ é diferenciável. Além disso, note que

$$
\|\varphi(y)[f(x-y+t u)-f(x-y)] / t\| \leq|\varphi(y)| \operatorname{Lip}(f)\|u\|
$$

logo segue da comutatividade da convolução e do Teorema da convergência dominada 3.2.10 1) que

$$
D_{g}(x) u=\lim _{t \rightarrow 0} \int_{\mathbb{R}^{n}} \varphi(y)[(f(x-y+t u)-f(x-y)) / t] d y=\int_{\mathbb{R}^{n}} \varphi(y) h_{u}(x-y) d y=\varphi * h_{u}(x)
$$

e assim

$$
\varphi *\left(h_{u+v}-h_{u}-h_{v}\right)=D_{g}(x)(u+v-u-v)=0
$$

para cada $u, v \in G$. Definindo para cada $k \in \mathbb{N}$ a função $\varphi_{k}: \mathbb{R}^{n} \rightarrow \mathbb{R}$ por $\varphi_{k}(x)=k^{n} \varphi(k x)$, obtemos uma sequência de funções satisfazendo as mesmas condições que $\varphi$ para cada $k \in \mathbb{N}$, isto é, $\varphi_{k}$ é uma função $C^{1}$ com suporte compacto e satisfaz

$$
\int_{\mathbb{R}^{n}} \varphi_{k}(x) d x=k^{n} \int_{\mathbb{R}^{n}} \varphi(k x) d x=k^{n} \frac{1}{k^{n}} \int_{\mathbb{R}^{n}} \varphi(x) d x=1 .
$$

Portanto a igualdade acima vale para toda $\varphi_{k}$. Vamos mostrar que $\lim _{k} \varphi_{k} * h(x)=h(x)$ para toda função limitada e mensurável $h: \mathbb{R}^{n} \rightarrow X$ e cada ponto de Lebesgue $x$ de $h$. De fato, seja $x$ um ponto de Lebesgue de $h$ e para cada $k \in \mathbb{N}$, defina $r(k)=\sup _{x \in \text { supp } \varphi}\|x\| / k$ e note que $\lim _{k} r(k)=0$. Veja que supp $\varphi_{k}=\frac{1}{k} \operatorname{supp} \varphi, \operatorname{logo} \operatorname{supp} \varphi_{k} \subset B(0 ; 2 r(k))$. Podemos supor sem perda de generalidade que $X$ é um espaço separável pois a imagem de $f$ o é. Logo, por 2.3.7, existe uma sequência $\left(x_{m}^{*}\right)_{m}$ em $S_{X^{*}}$ tal que $\|x\|=\sup _{m}\left|x_{m}^{*}(x)\right|$ para todo $x \in X$. Sejam $\epsilon>0$ e $k$ suficientemente grande tais que

$$
\frac{1}{m(B(0 ; 2 r(k)))} \int_{B(0 ; 2 r(k))}\|h(x+z)-h(x)\| d z<\epsilon .
$$

Temos para cada $m \in \mathbb{N}$ :

$$
x_{m}^{*}(h(x))=x_{m}^{*}(h(x)) \int_{B(0 ; 2 r(k))} \varphi_{k}(z) d z=\int_{B(0 ; 2 r(k))} x_{m}^{*}\left(h(x) \varphi_{k}(z)\right) d z .
$$


Segue então para cada $m \in \mathbb{N}$ que

$$
\begin{aligned}
\left|x_{m}^{*}(h(x))-x_{m}^{*}\left(\varphi_{k} * h(x)\right)\right| & =\left|\int_{B(0 ; 2 r(k))} x_{m}^{*}\left(h(x) \varphi_{k}(z)\right) d z-\int_{B(0 ; 2 r(k))} x_{m}^{*}\left(h(x+z) \varphi_{k}(z)\right) d z\right| \\
& =\left|\int_{B(0 ; 2 r(k))} \varphi_{k}(z) x_{m}^{*}(h(x+z)-h(x)) d z\right| \\
& \leq\left\|\varphi_{k}\right\|_{\infty} m(B(0 ; 2 r(k))) \frac{1}{m(B(0 ; 2 r(k)))} \int_{B(0 ; 2 r(k))}\left|x_{m}^{*}(h(x+z)-h(x))\right| d \\
& \leq\left\|\varphi_{k}\right\|_{\infty} m(B(0 ; 2 r(k))) \frac{1}{m(B(0 ; 2 r(k)))} \int_{B(0 ; 2 r(k))}\|h(x+z)-h(x)\| d z \\
& \leq k^{n}\|\varphi\|_{\infty} m(B(0 ; 2 r(k))) \epsilon
\end{aligned}
$$

pois $\left\|\varphi_{k}\right\|_{\infty} \leq k^{n}\|\varphi\|_{\infty}$. Mas para todo $r>0$, temos $m(B(0 ; r))=r^{n} C_{n}$, onde $C_{n}$ é uma constante dependendo apenas da dimensão $n$. Como $r(k)^{n}=\frac{1}{k^{n}}\left(\sup _{x \in \operatorname{supp} \varphi}\|x\|\right)^{n}$, segue que

$$
\left|x_{m}^{*}(h(x))-x_{m}^{*}\left(\varphi_{k} * h(x)\right)\right| \leq 2^{n} C_{n}\|\varphi\|_{\infty}\left(\sup _{x \in \text { supp } \varphi}\|x\|\right)^{n} \epsilon .
$$

Temos assim que

$$
\left\|h(x)-\varphi_{k} * h(x)\right\| \leq 2^{n} C_{n}\|\varphi\|_{\infty}\left(\sup _{x \in \operatorname{supp} \varphi}\|x\|\right)^{n} \epsilon,
$$

provando então que $h(x)=\lim _{k} \varphi_{k} * h(x)$. Segue então que

$$
\left(h_{u+v}-h_{u}-h_{v}\right)(x)=\lim _{k} \varphi_{k} *\left(h_{u+v}-h_{u}-h_{v}\right)(x)=0 .
$$

Portanto as derivadas direcionais em $x$ existem nas direções de $G$ para quase todo $x \in \mathbb{R}^{n}$ e $u \mapsto h_{u}(x)$ é aditiva em $u$ para quase todo $x \in \mathbb{R}^{n}$, e o resultado segue do Lema 3.3.7.

Teorema 3.3.13. Sejam $X$ e $Y$ espaços de Banach separáveis, onde $Y$ tem RNP. Então toda função $f: X \rightarrow Y$ lipschitziana é Gâteaux diferenciável a menos de um cojunto Haar nulo.

Demonstração. Seja $\left(F_{n}\right)_{n \in \mathbb{N}}$ uma sequência crescente de subespaços de $X$ de dimensão finita cuja união é densa em $X$. Definimos

$$
\begin{aligned}
& A_{n}=\left\{x \in X: \text { existe } T=T(n, x) \in \mathcal{L}\left(F_{n}, Y\right)\right. \text { tal que } \\
& \left.\qquad \lim _{t \rightarrow 0}(f(x+t v)-f(x)-t T(v)) / t=0 \text { para todo } v \in F_{n}\right\}
\end{aligned}
$$

e definimos também $B_{n}=X \backslash A_{n}$. Fixe uma base $\left(e_{1}, \ldots, e_{m}\right)$ para $F_{n}$ e sejam $\left(\xi_{1}, \ldots, \xi_{m}\right)$ variáveis aleatórias em $[0,1]$ independentes com distribuição uniforme. Por fim, definimos $\xi=\sum_{i=1}^{m} \xi_{i} e_{i}$. Queremos provar que $\mathbb{E}\left(\chi_{B_{n}}(\xi+x)\right)=0$ para todo $x \in X$, e portanto que $B_{n}$ é Haar nulo.

Veja que $\xi(t)+x \in B_{n}$ se e somente se $t \in \xi^{-1}\left[B_{n}-x\right], \operatorname{logo} \mathbb{E}\left(\chi_{B_{n}}(\xi+x)\right)=m\left(\xi^{-1}\left[B_{n}-x\right]\right)$. 
Defina a função $F: \mathbb{R}^{m} \rightarrow Y$ por

$$
F\left(t_{1}, \ldots, t_{m}\right)=f\left(\sum_{i=1}^{m} t_{i} e_{i}+x\right) \text { para cada }\left(t_{1}, \ldots, t_{m}\right) \in \mathbb{R}^{m}
$$

Veja que $F$ é Lipschitz pois

$$
\begin{aligned}
\left\|F\left(t_{1}, \ldots, t_{m}\right)-F\left(t_{1}^{\prime}, \ldots, t_{m}^{\prime}\right)\right\| & =\left\|f\left(\sum_{i=1}^{m} t_{i} e_{i}+x\right)-f\left(\sum_{i=1}^{m} t_{i}^{\prime} e_{i}+x\right)\right\| \\
& \leq \operatorname{Lip}(f) \max _{i=1, . ., m}\left\|e_{i}\right\| \max _{i=1, \ldots, m}\left|t_{i}-t_{i}^{\prime}\right| .
\end{aligned}
$$

para todos $\left(t_{1}, \ldots, t_{m}\right),\left(t_{1}^{\prime}, \ldots, t_{m}^{\prime}\right) \in R^{m}$. Definimos também o conjunto $C_{n} \subset \mathbb{R}^{m}$ por

$$
C_{n}=\left\{\left(t_{1}, \ldots, t_{m}\right) \in \mathbb{R}^{m}: F \text { não é diferenciável em }\left(t_{1}, \ldots, t_{m}\right)\right\}
$$

Note que $F$ é diferenciável em $\left(t_{1}, \ldots, t_{m}\right)$ se e somente se $\sum_{i=1}^{m} t_{i} e_{i}+x \in A_{n}$, logo

$$
\xi^{-1}\left[B_{n}-x\right]=\left\{t \in[0,1]: \xi(t)+x \notin A_{n}\right\}=\left(\xi_{1}, \ldots, \xi_{m}\right)^{-1}\left(C_{n}\right)
$$

Portanto $m\left(\xi^{-1}\left[B_{n}-x\right]\right)=\nu_{\xi_{1}, \ldots, \xi_{m}}\left(C_{n}\right)$, onde esta última é a distribuição da variável aleatória $\left(\xi_{1}, \ldots, \xi_{m}\right)$, que é o produto $\nu_{\xi_{1}} \otimes \ldots \otimes \nu_{\xi_{m}}\left(C_{n}\right)=m_{\mathbb{R}^{m}}\left(C_{n} \cap(0,1)\right)$ pois as variáveis aleatórias têm distribuição uniforme (veja 2.2.7). Mas pelo Teorema 3.3.11, $m_{\mathbb{R}^{m}}\left(C_{n}\right)=0$. Por fim, basta provar que conjunto dos pontos de $X$ nos quais $f$ é Gâteaux diferenciável é exatamente $\bigcap_{n \in \mathbb{N}} A_{n}$. É claro que se $f$ é diferenciável em $x$ então $x \in \bigcap_{n \in \mathbb{N}} A_{n}$. Por outro lado, se $x \in \bigcap_{n \in \mathbb{N}} A_{n}$, temos por 3.3.2 que

$$
T(n+1, x)(v)=T(n, x)(v) \text { para cada } v \in F_{n},
$$

dessa forma conseguimos definir $T: \bigcup_{n \in \mathbb{N}} F_{n} \rightarrow Y$ linear tal que $T(v)=T(n, x)(v)$ para cada $x \in F_{n}$ e para todo $n \in \mathbb{N}$ e assim

$$
T(v)=\lim _{t \rightarrow 0}(f(x+t v)-f(x)) / t
$$

Segue da densidade de $\bigcup_{n \in \mathbb{N}} F_{n}$ e do Lema 3.3.7 que $f$ é Gâteaux diferenciável em $x$.

Definição 3.3.14. Sejam $M_{1}, M_{2}$ espaços métricos e $f: M_{1} \rightarrow M_{2}$ uma função. Dizemos que $f$ é um mergulho Lipschitz se existem constantes $A, B>0$ tais que

$$
A d(x, y) \leq d(f(x), f(y)) \leq B d(x, y) \text { para todos } x, y \in M_{1}
$$

Teorema 3.3.15. (Heinrich-Mankiewicz) Sejam X e $Y$ espaços de Banach separáveis e 
suponha que exista um mergulho Lipschitz de $X$ em $Y$. Assuma que $Y$ possua RNP. Então $X$ pode ser mergulhado linearmente em $Y$.

Demonstração. Seja $f: X \rightarrow Y$ um mergulho Lipschitz e $c_{1}, c_{2}>0$ tais que

$$
c_{1}\|x-y\| \leq\|f(x)-f(y)\| \leq c_{2}\|x-y\| .
$$

Como $f$ é Gâteaux diferenciável quase sempre, existe $a \in X$ tal que $f$ é diferenciável em $a$. Seja $T$ a derivada de $f$ em $a$. Então, escrevendo $T(x)=f(a+x)-f(a)-r(x)$, teremos para qualquer $x$ :

$$
c_{1}\|x\|-\|r(x)\| \leq\|T(x)\| \leq c_{2}\|x\|+\|r(x)\|
$$

e portanto, para cada $t \neq 0$ :

$$
c_{1} \frac{\|t x\|}{|t|}-\frac{\|r(t x)\|}{|t|} \leq \frac{\|T(t x)\|}{|t|} \leq c_{2} \frac{\|t x\|}{|t|}+\frac{\|r(t x)\|}{|t|} .
$$

donde segue que:

$$
c_{1}\|x\| \leq\|T(x)\| \leq c_{2}\|x\|
$$

\subsection{Teorema de Rademacher fraco estrela}

Para garantir que funções Lipschitz $f: X \rightarrow Y$ fossem diferenciáveis, pedimos na seção anterior que $Y$ tivesse a propriedade de Radon Nikodým. Nesta seção iremos introduzir o conceito de diferenciabilidade fraca* e vamos mostrar que se $Y$ for um espaço dual, podemos garantir a diferenciabilidade fraca* para certos pontos em $X$. Vamos mostrar em particular que a partir de todo mergulho Lipschitz de $X$ em um espaço de Banach $Y$ podemos construir um mergulho linear de $X$ em $Y^{* *}$.

Definição 3.4.1. Sejam $X, Z$ espaços de Banach e $Y=Z^{*}$. Seja $f: X \rightarrow Y$ uma função Lipschitz. Dizemos que $f$ é fraca*-diferenciável em $x_{0} \in X$ se para todo $x \in X$ existe o limite

$$
w^{*}-\lim _{t \rightarrow 0} \frac{f\left(x_{0}+t x\right)-f(x)}{t}=\left(D^{*} f\right)_{x_{0}}(x)
$$

na topologia fraca* de $Y$ e a função $\left(D^{*} f\right)_{x_{0}}(x)$ é linear em $x$.

Lema 3.4.2. Seja $X$ um espaço normado. Sejam $f: \mathbb{N} \rightarrow X^{*}$ uma sequência fraca*-Cauchy e $g: J \rightarrow \mathbb{N}$ uma rede tais que $f \circ g$ é uma subrede convergente de $f$. Então $f$ é uma sequência convergente.

Demonstração. Seja $x^{*} \in X^{*}$ tal que $x^{*}=w^{*}-\lim _{\beta \in J} f \circ g(\beta)$. Vamos mostrar que $x^{*}=$ $\lim _{n \in \mathbb{N}} f(n)$. Sejam $\epsilon>0$ e $x \in X$. Então existe $N \in \mathbb{N}$ tal que $k, l \geq N$ implica $\mid f(k)(x)-$ 
$f(l)(x) \mid<\epsilon$. Além disso, existe $\beta_{0} \in J$ tal que $\beta_{0} \preceq \beta$ implica $\left|f \circ g(\beta)(x)-x^{*}(x)\right|<\epsilon$. Como $f \circ g$ é uma subrede, existe $\beta_{1} \in J$ tal que $g\left(\beta_{1}\right) \geq N$ e $\beta_{0} \preceq \beta_{1}$. Então

$$
\left|x^{*}(x)-f(n)(x)\right| \leq\left|x^{*}(x)-f\left(g\left(\beta_{1}\right)\right)(x)\right|+\left|f\left(g\left(\beta_{1}\right)\right)(x)-f(n)(x)\right|<2 \epsilon \text { para todo } n \geq N \text {. }
$$

Segue da arbitrariedade de $x \in X$ que $f$ é uma sequência fraca* convergente a $x^{*}$.

Teorema 3.4.3. Sejam $X$ um espaço de Banach de dimensão finita, $Z$ um espaço de Banach separável e $f: X \rightarrow Z^{*}$ uma função Lipschitz. Então

1. $\left(D^{*} f\right)_{x}$ existe para quase todo $x \in X$.

2. Se $\|f(x)-f(y)\| \leq K\|x-y\|$ para todos $x, y \in X$, então $\left\|\left(D^{*} f\right)_{x}\right\| \leq K$ sempre que existir $\left(D^{*} f\right)_{x}$.

3. Se $k\|x-y\| \leq\|f(x)-f(y)\| \leq K\|x-y\|$ para todos $x, y \in X$, então para quase todo $x \in X,\left(D^{*} f\right)_{x}$ é um mergulho linear de $X$ em $Z^{*}$ com $\left\|\left(D^{*} f\right)_{x}^{-1}\right\| \leq k^{-1}$.

Demonstração. 1) Sejam $\left\{z_{n}: n \in \mathbb{N}\right\}$ um subconjunto denso da esfera unitária $S_{Z}$ e para cada $n \in \mathbb{N}$, defina $\varphi_{n}: X \rightarrow \mathbb{R}$ por $\varphi_{n}(x)=f(x)\left(z_{n}\right)$ para todo $x \in X$. Pelo teorema de Rademacher, cada $\varphi_{n}$ é diferenciável quase sempre e assim o complementar do conjunto $W=\left\{x \in X:\left(D \varphi_{n}\right)_{x}\right.$ existe para cada $\left.n \in \mathbb{N}\right\}$ tem medida nula. Afirmamos que $\left(D^{*} f\right)_{x}$ existe para todo $x \in W$. De fato, seja $x \in W, a \in X$ e $n \in \mathbb{N}$. Por definição temos que

$$
\left(D \varphi_{n}\right)_{x}(a)=\lim _{t \rightarrow 0} \frac{\varphi_{n}(x+t a)-\varphi_{n}(x)}{t}=\lim _{t \rightarrow 0} \frac{f(x+t a)-f(x)}{t}\left(z_{n}\right) .
$$

Seja $\left(t_{n}\right)_{n \in \mathbb{N}}$ uma sequência de números reais convergindo a 0 . Definindo para cada $k \in \mathbb{N}$ o funcional $f_{k}: Z \rightarrow \mathbb{R}$ por $f_{k}=t_{k}^{-1}\left(f\left(x+t_{k} a\right)-f(x)\right)$, temos uma sequência $\left(f_{k}\right)_{k}$ fraca*Cauchy. De fato, temos

$$
\begin{aligned}
\left\|f_{k}\right\|=\sup _{z \in S_{Z}}\left|f_{k}(z)\right| & =\sup _{z \in S_{Z}}\left|\frac{f\left(x+t_{k} a\right)-f(x)}{t_{k}}(z)\right| \\
& \leq\left\|\frac{f\left(x+t_{k} a\right)-f(x)}{t_{k}}\right\| \\
& \leq \operatorname{Lip}(f)\|a\|
\end{aligned}
$$

para todo $k \in \mathbb{N}$ e assim, para cada $z \in S_{Z}$ temos que

$\left\|\left(f_{k}-f_{l}\right)(z)\right\| \leq\left\|f_{k}-f_{l}\right\|\left\|z-z_{n}\right\|+\left\|\left(f_{k}-f_{l}\right)\left(z_{n}\right)\right\| \leq 2 \operatorname{Lip}(f)\|a\|\left\|z-z_{n}\right\|+\left\|\left(f_{k}-f_{l}\right)\left(z_{n}\right)\right\|$.

Como $\left\{z_{n}: n \in \mathbb{N}\right\}$ é denso em $S_{Z}$, dado $z \in S_{Z}$ tome $n_{0} \in \mathbb{N}$ tal que $\left\|z-z_{n_{0}}\right\|<$ $\epsilon(2 \operatorname{Lip}(f)\|a\|)^{-1}$. Mas como

$$
\lim _{k \in \mathbb{N}} f_{k}\left(z_{n_{0}}\right)=\lim _{k \in \mathbb{N}} \frac{f\left(x+t_{k} a\right)-f(x)}{t_{k}}\left(z_{n_{0}}\right)=\lim _{t \rightarrow 0} \frac{f(x+t a)-f(x)}{t}\left(z_{n_{0}}\right)=\left(D \varphi_{n_{0}}\right)_{x}(a),
$$


segue que $\left(f_{k}\left(z_{n_{0}}\right)\right)_{k}$ é Cauchy e então existe $N$ tal que $k, l \geq N$ implicam $\left\|\left(f_{k}-f_{l}\right)\left(z_{n_{0}}\right)\right\|<\epsilon$. Assim

$$
\left\|\left(f_{k}-f_{l}\right)(z)\right\| \leq 2 \operatorname{Lip}(f)\|a\|\left\|z-z_{n_{0}}\right\|+\left\|\left(f_{k}-f_{l}\right)\left(z_{n_{0}}\right)\right\|<\epsilon+\epsilon=2 \epsilon .
$$

Isto prova que $\left(f_{k}\right)_{k}$ é fraca*-Cauchy.

Por 2.3.25, toda sequência Cauchy em um espaço vetorial topológico é limitada, segue então do Teorema de Banach-Alaoglu 2.3.32 e da Proposição 2.3.44 que existe uma subrede convergente na topologica fraca*. Então a sequência $\left(f_{k}\right)_{k}$ é fraca*-convergente por 3.4.2. Segue da generalidade de $\left(t_{k}\right)_{k}$ que o limite

$$
w^{*}-\lim _{t \rightarrow 0} \frac{f(x+t a)-f(x)}{t}
$$

existe para todo $a \in X$ e logo existe $\left(D^{*} f\right)_{x}$ para todo $x \in W$. Vamos mostrar que $\left(D^{*} f\right)_{x}$ é linear. Sejam $a, b \in X$ e $\lambda \in \mathbb{R}$. Se $n \in \mathbb{N}$, então

$$
\begin{aligned}
\left(D^{*} f\right)_{x}(a+\lambda b)\left(z_{n}\right) & =\lim _{t \rightarrow 0} \frac{f(x+t(a+\lambda b))-f(x)}{t}\left(z_{n}\right) \\
& =\left(D \varphi_{n}\right)_{x}(a+\lambda b) \\
& =\left(D \varphi_{n}\right)_{x}(a)+\lambda\left(D \varphi_{n}\right)_{x}(b) \\
& =\left(D^{*} f\right)_{x}(a)\left(z_{n}\right)+\lambda\left(D^{*} f\right)_{x}(b)\left(z_{n}\right) .
\end{aligned}
$$

Como $\left\{z_{n}: n \in \mathbb{N}\right\}$ é denso em $S_{X}$, temos então que

$$
\left(D^{*} f\right)_{x}(a+\lambda b)=\left(D^{*} f\right)_{x}(a)+\lambda\left(D^{*} f\right)_{x}(b) .
$$

2) Sejam $x \in W$ e $a \in X$. Então

$$
\left\|\left(D^{*} f\right)_{x}(a)\right\|=\sup _{n}\left|\left(D^{*} f\right)_{x}(a)\left(z_{n}\right)\right|=\sup _{n}\left|\lim _{t \rightarrow 0} \frac{f(x+t a)-f(x)}{t}\left(z_{n}\right)\right| \leq K\|a\| .
$$

3) Suponha sem perda de generalidade que $k=1$. Afirmamos que o complementar do conjunto $M_{a}=\left\{x \in W:\left\|\left(D^{*} f\right)_{x}(a)\right\| \geq 1\right\}$ tem medida nula para todo $a \in X$ com $\|a\|=1$. Caso, contrário, como

$$
M_{a}^{c}=\bigcup_{m}\left\{x \in W:\left\|\left(D^{*} f\right)_{x}(a)\right\|<1-1 / m\right\}
$$

existe $m \in \mathbb{N}$ tal que $N=\left\{x \in W:\left\|\left(D^{*} f\right)_{x}(a)\right\|<1-1 / m\right\}$ tem medida positiva. Como fizemos no Lema 3.3.12, temos que

$$
m_{\mathbb{R}^{n}}(N)=\alpha \int_{\mathbb{R}} \ldots \int_{\mathbb{R}} \chi_{N}\left(J\left(0, x_{2}, \ldots, x_{n}\right)+x_{1} a\right) d m_{\mathbb{R}}\left(x_{1}\right) d m_{\mathbb{R}}\left(x_{2}\right) \ldots d m_{\mathbb{R}}\left(x_{n}\right)
$$


para algum $\alpha \in \mathbb{R}$ e $J: \mathbb{R}^{n} \rightarrow \mathbb{R}^{n}$ isomorfismo linear. Então existem $x_{2}, \ldots, x_{n} \in \mathbb{R}$ para os quais

$$
m_{\mathbb{R}}\left(\left\{t \in \mathbb{R}: x_{0}+t a \in N\right\}\right)=\int_{\mathbb{R}} \chi_{N}\left(x_{0}+x_{1} a\right) d m_{\mathbb{R}}\left(x_{1}\right)>0,
$$

onde $x_{0}=J\left(0, x_{2}, \ldots, x_{n}\right)$. Seja $t_{0} \in\left\{t \in \mathbb{R}: x_{0}+t a \in N\right\}$ um ponto de Lebesgue da função $t \mapsto \chi_{N}\left(x_{0}+t a\right)$. Então

$$
\lim _{r \rightarrow 0^{+}} r^{-1}\left|\int_{t_{0}}^{t_{0}+r}\left(\chi_{N}\left(x_{0}+y a\right)-\chi_{N}\left(x_{0}+t_{0} a\right)\right) d y\right|=0 .
$$

Mas então

$$
\lim _{r \rightarrow 0^{+}} r^{-1} \int_{t_{0}}^{t_{0}+r} \chi_{N}\left(x_{0}+y a\right) d y=\lim _{r \rightarrow 0^{+}} r^{-1} \int_{t_{0}}^{t_{0}+r} \chi_{N}\left(x_{0}+t_{0} a\right) d y=1
$$

Isto implica que existe $r>0$ tal que

$$
\int_{t_{0}}^{t_{0}+r} \chi_{N}\left(x_{0}+y a\right) d y>r(1-1 /(2 K m)) \text {. }
$$

Mas esta integral é justamente $m_{\mathbb{R}}\left(\left\{t \in\left(t_{0}, t_{0}+r\right): x_{0}+t a \in N\right\}\right)$, que por sua vez, como a medida de Lebesgue é invariante por translações, é $m_{\mathbb{R}}\left(\left\{t \in(0, r): x_{0}+\left(t_{0}+t\right) a \in N\right\}\right)$. Definimos $\bar{x}=x_{0}+t_{0} a, A=\{t \in(0, r): \bar{x}+t a \in N\}$ e $B=(0, r) \backslash A$. Temos

$$
\sup _{z \in S_{Z}}|(f(\bar{x}+r a)-f(\bar{x}))(z)|=\|f(\bar{x}+r a)-f(\bar{x})\| \geq r\|a\|=r
$$

então existe $z \in S_{Z}$ com

$$
|\varphi(r)-\varphi(0)|>r(1-1 /(2 K m))
$$

onde $\varphi(t)=f(\bar{x}+t a)(z)$ para todo $t \in[0, r]$. Temos que $\operatorname{Lip}(\varphi) \leq K$ e assim $\varphi^{\prime}(t)$ existe quase sempre e $\left|\varphi^{\prime}(t)\right| \leq K$ em $(0, r)$.

Se $t \in A$, em particular, $\bar{x}+t a \in W$, e assim

$$
\varphi^{\prime}(t)=\lim _{h \rightarrow 0} \frac{\varphi(t+h)-\varphi(t)}{h}=\lim _{h \rightarrow 0} \frac{f(\bar{x}+(t+h) a)-f(\bar{x}+t a)}{h}(z)=\left(D^{*} f\right)_{\bar{x}+t a}(a)(z) .
$$


Temos então

$$
\begin{aligned}
r(1-1 /(2 m))<|\varphi(r)-\varphi(0)| & =\left|\int_{(0, r)} \varphi^{\prime}(t) d t\right| \\
& \leq\left|\int_{A}\left(D^{*} f\right)_{\bar{x}+t a}(a)(z) d t\right|+\int_{B} K d t \\
& \leq \int_{A}\left\|\left(D^{*} f\right)_{\bar{x}+t a}(a)\right\| d t+K r /(2 K m) \\
& \leq r(1-1 / m)+r /(2 m)=r(1-1 /(2 m)),
\end{aligned}
$$

uma contradição.

Teorema 3.4.4. Sejam $X$ e $Z$ espaços de Banach separáveis. Se existir um mergulho Lipschitz de $X$ em $Z^{*}$, então $X$ é isomorfo a um subespaço de $Z^{*}$.

Demonstração. Seja $f: X \rightarrow Z^{*}$ um mergulho Lipschitz. A menos de um conjunto Haar nulo em $X$, existe $\left(D^{*} f\right)_{x}$. De fato, assim como em 3.3, podemos tomar uma sequência crescente de subespaços de dimensão finita $F_{n}$ cuja união $\bigcup_{n \in \mathbb{N}} F_{n}$ é densa em $X$ e definimos

$$
\begin{aligned}
& A_{n}=\left\{x \in X: \text { existe } T=T(n, x) \in \mathcal{L}\left(F_{n}, Z^{*}\right)\right. \text { tal que } \\
& \left.\quad T(v)=w^{*}-\lim _{t \rightarrow 0}(f(x+t v)-f(x)) / t \text { para todo } v \in F_{n} \text { e } T \text { é um mergulho linear }\right\} .
\end{aligned}
$$

Segue como fizemos no Teorema 3.3 que $\left(D^{*} f\right)_{x}$ existe e é um mergulho linear para todo $x \in \bigcap_{n \in \mathbb{N}} A_{n}$.

Queremos mostrar que o resultado acima ainda vale se não nos limitarmos a um espaço $Z$ separável. Para isso, precisamos de um resultado da teoria de modelos.

Teorema 3.4.5 (Lowenheim-Skolem). Seja $X$ um espaço de Banach e $Y \subset X$ um subespaço não trivial. Então existem um outro subespaço $Z \subset X$ contendo $Y$, com dens $Z=$ dens $Y$, um ultrafiltro $\mathcal{U}$ em $\mathbb{N}$ e uma isometria $S: Z_{\mathcal{U}} \rightarrow X_{\mathcal{U}}$ que façam o diagrama abaixo comutar

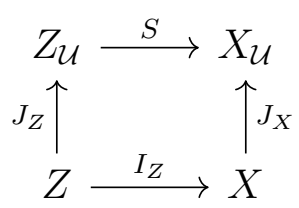

isto é, $S \circ J_{Z}=J_{X} \circ I_{Z}$, onde $J_{Z}: Z \rightarrow Z_{\mathcal{U}}, J_{X}: X \rightarrow X_{\mathcal{U}}$ e $I_{Z}: Z \rightarrow X$ são as inclusões canônicas.

Demonstração. Veja [Ste78] Teorema 2.2.

Proposição 3.4.6. Sejam $X$ um espaço de Banach e $Y \subset X$ um subespaço não trivial. Então existem um outro subespaço $Z \subset X$ contendo $Y$, com dens $Z=$ dens $Y$ e um mergulho isométrico $T: Z^{*} \rightarrow X^{*}$ tal que $T\left(z^{*}\right)(z)=z^{*}(z)$ para todo $z \in Z, z^{*} \in Z^{*}$. 
Demonstração. Sejam $Z \subset X$ subespaço, $\mathcal{U}$ um ultrafiltro em $\mathbb{N}$ e $S: Z_{\mathcal{U}} \rightarrow X_{\mathcal{U}}$ isometria como no Teorema de Lowenheim-Skolem. Definimos $T: Z^{*} \rightarrow X^{*}$ por

$$
T=\left(J_{X}\right)^{*} \circ\left(S^{-1}\right)^{*} \circ J_{Z^{*}}
$$

onde $J_{Z^{*}}: Z^{*} \rightarrow\left(Z_{\mathcal{U}}\right)^{*}$ é a inclusão $J_{Z^{*}}\left(z^{*}\right)\left(\overline{\left(z_{n}\right)_{n \in \mathbb{N}}}\right)=\lim _{n \in \mathcal{U}} z^{*}\left(z_{n}\right)$ e $J_{X}: X \rightarrow X_{\mathcal{U}}$ é as inclusão canônica. Sejam $z \in Z$ e $z^{*} \in Z^{*}$. Então

$$
\begin{aligned}
T\left(z^{*}\right)(z)=\left(J_{X}\right)^{*} \circ\left(S^{-1}\right)^{*} \circ J_{Z^{*}}\left(z^{*}\right)(z) & =\left(S^{-1}\right)^{*}\left(J_{Z^{*}}\left(z^{*}\right)\right)\left(J_{X}(z)\right) \\
& =J_{Z^{*}}\left(z^{*}\right)\left(S^{-1}\left(J_{X}(z)\right)\right) \\
& =J_{Z^{*}}\left(z^{*}\right)\left(J_{Z}(z)\right) \\
& =\lim _{n \in \mathcal{U}} z^{*}(z) \\
& =z^{*}(z) .
\end{aligned}
$$

T é isometria pois é uma composição de isometrias. Isto conclui a prova.

Definição 3.4.7. Seja $X$ um espaço de Banach, $Y \subset X$ e $Z \subset X^{*}$ subespaços. Dizemos que $Y$ é um espaço normante para $Z$ se $\sup _{y \in B_{Y}}|z(y)|$ define uma norma equivalente à norma padrão em $Z$.

Lema 3.4.8. Se $Z$ é um subespaço separável de $X^{*}$ então existe $Y$ subespaço separável de $X$ que é um espaço normante para $Z$.

Demonstração. Sejam $\left(z_{n}\right)_{n \in \mathbb{N}}$ uma sequência densa em $S_{Z}$ e $x_{n} \in S_{X}$ tais que $\left|z_{n}\left(x_{n}\right)\right|>\frac{n}{n+1}$ para cada $n \in \mathbb{N}$. Para cada $z \in S_{Z}$, seja $\left(z_{n_{k}}\right)_{k}$ subsequência convergindo a $z$. Temos assim, para $k$ suficientemente grande, $\frac{n_{k}}{n_{k}+1}>3 / 4$ e $\left\|z-z_{n_{k}}\right\|<1 / 4$. Então

$$
\left|z\left(x_{n_{k}}\right)\right|>\left|z_{n_{k}}\left(x_{n_{k}}\right)\right|-\left\|z-z_{n_{k}}\right\|>3 / 4-1 / 4=1 / 2 .
$$

Tomando $Y=\left[\left\{x_{n}: n \in \mathbb{N}\right\}\right]$, segue que

$$
1 / 2\|z\| \leq \sup _{y \in B_{Y}}|z(y)| \leq\|z\| \text { para todo } z \in Z
$$

Podemos finalmente provar o resultado mais geral.

Teorema 3.4.9. Sejam $X$ um espaço de Banach separável, $Z$ um espaço de Banach e $f: X \rightarrow Z^{*}$ um mergulho Lipschitz. Então $X$ é isomorfo a um subespaço de $Z^{*}$.

Demonstração. Seja $Z_{0} \subset Z$ um subespaço separável de $Z$ que é um espaço normante para $[f[X]]$. Pela proposição acima, existe $Z_{1}$ separável com $Z_{0} \subset Z_{1} \subset Z$ e $Z_{1}^{*}$ é mergulhado isometricamente em $Z^{*}$. Seja $Q: Z^{*} \rightarrow Z_{1}^{*}$ a função de restrição a $Z_{1}$. Como $Z_{0}$ é normante 
para $f[X]$, assim é $Z_{1}$ e logo $Q \circ f$ é um mergulho Lipschitz de $X$ em $Z_{1}^{*}$. De fato, existe $c<1$ tal que para cada $x \in X$, temos

$$
\|Q(f(x))\| \geq c\|f(x)\| \geq c \operatorname{Lip}(f)^{-1}\|x\| .
$$

Podemos aplicar então o Teorema 3.4.4 para obter que $X$ é isomorfo a um subespaço de $Z_{1}^{*}$, que por sua vez é isométrico a um subespaço de $Z^{*}$.

Corolário 3.4.10. Sejam $X$ e $Y$ espaços de Banach, $X$ separável, e suponha que exista um mergulho Lipschitz de $X$ em $Y$. Então X pode ser linearmente mergulhado em $Y^{* *}$.

\subsection{Isomorfismos Lipschitz entre espaços Reflexivos}

Até agora, conseguimos mergulhar linearmente um espaço de Banach $X$ em um espaço $Y$ com RNP que é Lipschitz isomorfo a $X$. Nesta seção, vamos mostrar que se esses espaços são reflexivos, este mergulho vai ter como imagem um subespaço complementado.

Definição 3.5.1. Seja $G$ um conjunto. Definimos $\ell_{\infty}(G)$ como sendo o espaço de todas funções $f: G \rightarrow \mathbb{R}$ limitadas. Definimos ainda para cada $f \in \ell_{\infty}(G)$

$$
\|f\|_{\infty}=\sup _{g \in G}|f(g)|
$$

Observação 3.5.2. A função $\|\cdot\|_{\infty}: \ell_{\infty}(G) \rightarrow[0, \infty)$ é uma norma em $\ell_{\infty}(G)$.

Definição 3.5.3. Seja $G$ um grupo. Uma média invariante em $G$ é um funcional linear $M$ em $\ell_{\infty}(G)$ tal que:

1. $M\left(1_{\ell_{\infty}(G)}\right)=1$, onde $1_{\ell_{\infty}(G)}: G \rightarrow \mathbb{R}$ é a função dada por $1_{\ell_{\infty}(G)}(g)=1$ para todo $g \in G$.

2. $\|M\|=1$.

3. $M\left(f_{g}\right)=M(f)$ para cada $g \in G$, onde $f_{g}(x)=f(g x)$ para todo $x \in X$.

Observação 3.5.4. Seja $G$ um grupo e $M \in\left(\ell_{\infty}(G)\right)^{*}$. Note que se $M\left(1_{\ell_{\infty}(G)}\right)=1$ e $M(f) \geq$ 0 para cada $f \geq 0$, então $\|M\|=1$. De fato, como $|f|-f \geq 0$ e $\|f\|_{\infty}-|f| \geq 0$ para cada $f \in \ell_{\infty}(G)$, temos

$$
|M(f)| \leq M(|f|) \leq\|f\|_{\infty} M\left(\left(1_{\ell_{\infty}(G)}\right)=\|f\|_{\infty} .\right.
$$

Logo $\|M\| \leq 1$ e como $M\left(\left(1_{\ell_{\infty}(G)}\right)=1\right.$, segue que $\|M\|=1$.

Definição 3.5.5. Sejam $X$ um espaço vetorial topológico e $W \subset X$ convexo. Dizemos que uma função $T: W \rightarrow X$ é afim se

$$
T\left(t w_{1}+(1-t) w_{2}\right)=t T\left(w_{1}\right)+(1-t) T\left(w_{2}\right) \text { para todos } w_{1}, w_{2} \in W \text { e } t \in[0,1] .
$$


Definição 3.5.6. Sejam $X$ um espaço vetorial topológico e $W \subset X$ um compacto convexo. Para cada função $T: W \rightarrow W$, definimos $F_{T}=\{w \in W: T(w)=w\}$.

Observação 3.5.7. Se $T: W \rightarrow W$ é uma função contínua e convexa, então $F_{T}$ é um compacto convexo de $X$. De fato, como $F_{T}=(T-I d)^{-1}[\{0\}]$ e $T$ é contínua, segue que $F_{T}$ é um subconjunto fechado do compacto $W$, logo é compacto também. Além disso, dados $w_{1}, w_{2} \in F_{T}$ e $t \in[0,1]$, temos que

$$
T\left(t w_{1}+(1-t) w_{2}\right)=t T\left(w_{1}\right)+(1-t) T\left(w_{2}\right)=t w_{1}+(1-t) w_{2} \in W
$$

pois $W$ é um conjunto convexo.

Lema 3.5.8. Sejam $X$ um espaço vetorial topológico e $W \subset X$ um compacto convexo. Sejam $n \in \mathbb{N}$ e $T_{i}: W \rightarrow W$ uma função contínua e afim para cada $i=1, \ldots, n$. Se $T_{i} \circ T_{j}=T_{j} \circ T_{i}$ para cada $i \neq j$, então

$$
\bigcap_{i=1}^{n} F_{T_{i}} \neq \emptyset .
$$

Demonstração. Considere primeiro $T: W \rightarrow W$ uma função contínua e afim. Vamos mostrar que $F_{T} \neq \emptyset$. De fato, fixado $x \in W$, defina $x_{n}=\left(\sum_{i=0}^{n-1} T^{i} x\right) / n$. Como $T$ é afim, temos que

$$
T\left(x_{n}\right)=T\left(\frac{\sum_{i=0}^{n-1} T^{i} x}{n}\right)=\frac{\sum_{i=0}^{n-1} T^{i+1} x}{n}
$$

Como $W$ é compacto por 2.3.23, $W$ é um subconjunto limitado de $X$. Assim, para cada aberto $U$ de 0 , existe $s_{U}>0$ tal que $W \subset t U$ para cada $t>s_{U}$. Em particular, $\frac{1}{n} W \subset U$ para $n>s_{U}$ e isto prova que $\lim _{n \in \mathbb{N}} x / n=0$ e $\lim _{n \in \mathbb{N}} T^{n}(x) / n=0$. Temos então que

$$
\lim _{n \in \mathbb{N}}\left(T\left(x_{n}\right)-x_{n}\right)=\lim _{n \in \mathbb{N}}\left(T^{n}(x)-x\right) / n=0 .
$$

Como $W$ é compacto e $T$ contínua, existe um ponto de acumulação $y$ da sequência $\left(x_{n}\right)_{n \in \mathbb{N}}$ e temos $T(y)=y, \log o F_{T} \neq \emptyset$. Além disso, pela observação acima $F_{T}$ é convexo e compacto. Note que para qualquer $S: W \rightarrow W$ contínua e afim que comute com $T$, temos

$$
T(S(x))=S(T(x))=S(x) \text { para todo } x \in F_{T},
$$

$\operatorname{logo} F_{T}$ é $S$-invariante e aplicando o primeiro argumento para $W=F_{T}$, segue que existe $w \in F_{T}$ tal que $S(w)=w$, isto é, $w \in F_{T} \cap F_{S}$. Assim, vale

$$
\bigcap_{i=1}^{n} F_{T_{i}} \neq \emptyset
$$


para qualquer sequência finita de operadores $T_{i}: W \rightarrow W, i=1, \ldots n$, onde $T_{i}$ é contínuo, afim e $T_{i} \circ T_{j}=T_{j} \circ T_{i}$ para $i \neq j$.

Teorema 3.5.9. Se Gé um grupo abeliano, então $G$ admite uma média invariante.

Demonstração. Vamos equipar o espaço $\ell_{\infty}(G)^{*}$ com a topologia fraca*. Definimos

$$
W=\left\{\phi \in \ell_{\infty}(G)^{*}: \phi(f) \geq 0 \text { se } f \geq 0 \text { e } \phi\left(1_{\ell_{\infty}(G)}\right)=1\right\} \subset S_{\ell_{\infty}(G)^{*}} .
$$

Note que $W$ é convexo e também é compacto pois é fechado. Dado $g \in G$, definimos $T_{g}$ : $W \rightarrow W$ por $T_{g}(\phi)(f)=\phi\left(f_{g}\right)$. Temos que $T_{g}$ é afim e se $\left(\phi_{\alpha}\right)_{\alpha}$ é uma rede convergindo na topologia fraca* de $W$ para $\phi$, então

$$
T_{g}\left(\phi_{\alpha}\right)(f)=\phi_{\alpha}\left(f_{g}\right) \rightarrow \phi\left(f_{g}\right)=T_{g}(\phi)(f)
$$

para cada $f \in \ell_{\infty}(G)$, logo $T_{g}\left(\phi_{\alpha}\right)$ converge para $T_{g}(\phi)$ na topologia fraca* e $T_{g}$ é contínua. Pelo que vimos acima, o conjunto $F_{g}$ definido por $F_{T_{g}}$ é não vazio para cada $g \in G$. Veja que

$$
T_{g} \circ T_{h}=T_{g h}=T_{h g}=T_{h} \circ T_{g}
$$

pois $G$ é abeliano, portanto a família $\left\{F_{T_{g}}: g \in G\right\}$ de subconjuntos fechados de $W$ tem a propriedade da interseção finita e logo existe $M \in \bigcap\left\{F_{T_{g}}: g \in G\right\}$. Em particular $M(1)=1$, $M(f) \geq 0$ se $f \geq 0$ e para qualquer $g \in G$,

$$
M\left(f_{g}\right)=T_{g}(M)(f)=M(f) .
$$

Definição 3.5.10. Seja $X$ um espaço normado. Definimos Lip $p_{0}(f)$ como sendo o espaço de todas as funções Lipschitzianas $f: X \rightarrow \mathbb{R}$ tais que $f(0)=0$.

Observação 3.5.11. O espaço Lip $p_{0}$ é um espaço normado munido da norma $\|\cdot\|: \operatorname{Lip}_{0}(X) \rightarrow$ $[0, \infty]$ dada por $\|f\|=\operatorname{Lip}(f)$ pois se Lip $(f)=0$, então $f(x)=f(0)=0$ para todo $x \in X$.

Proposição 3.5.12. Se X é um espaço de Banach. Então existe uma projeção $P$ de Lip $p_{0}(X)$ em $X^{*}$. Além disso, dado um subespaço $E$ de $X$, tal projeção pode ser definida com a propriedade de que se $\left.f\right|_{E}$ é linear então $\left.P(f)\right|_{E}=\left.f\right|_{E}$ para toda $f \in \operatorname{Lip}_{0}(X)$.

Demonstração. Dado qualquer subespaço $Y$ de $X$, seja $M_{Y}$ uma média invariante em $(Y,+)$ e defina $Q_{Y}: \operatorname{Lip}_{0}(X) \rightarrow \operatorname{Lip}_{0}(X)$ por

$$
Q_{Y}(f)(x)=M_{Y}\left((f(x+u)-f(u))_{u \in E}\right) .
$$

Seja $f \in \operatorname{Lip}_{0}(X)$. Veja que para todos $x, y \in X$, temos

$$
\left|Q_{Y}(f)(x)-Q_{Y}(f)(y)\right| \leq\left\|M_{Y}\right\|\left\|(f(x+u)-f(y+u))_{u \in Y}\right\|_{\infty} \leq \operatorname{Lip}(f)\|x-y\|,
$$


$\operatorname{logo} Q_{Y}(f) \in \operatorname{Lip}_{0}(X)$. Como $Q_{Y}$ é linear, também segue que $\left\|Q_{Y}\right\| \leq 1$. Temos para cada $f \in X^{*}$

$$
Q_{Y}(f)(x)=M_{Y}\left((f(x))_{u \in Y}\right)=f(x) M_{Y}(1)=f(x)
$$

$\operatorname{assim} Q_{Y}$ fixa os pontos em $X^{*}$.

Se $u \in Y$, então para todo $x \in X$ temos

$$
\begin{aligned}
Q_{Y}(f)(x+u) & =M_{Y}\left((f(x+u+v)-f(v))_{v \in Y}\right) \\
& =M_{Y}\left((f(x+u+v)-f(u+v))_{v \in Y}\right)+M_{Y}\left((f(u+v)-f(v))_{v \in Y}\right) \\
& =Q_{Y}(f)(x)+Q_{Y}(f)(u) .
\end{aligned}
$$

Em particular, temos que

$$
Q_{X}(f)(x+y)=Q_{X}(f)(x)+Q_{X}(f)(y) \text { para todos } x, y \in X
$$

Fixamos um subespaço $E$ de $X$ e definimos $P=Q_{X} \circ Q_{E}$. Então, pelo que vimos acima, $P$ é uma transformação linear contínua e $P(f)=f$ para cada $f \in X^{*}$. Se $\left.f\right|_{E}$ é linear e $u \in E$, temos

$$
Q_{E}(f)(u)=M_{E}\left((f(u))_{v \in E}\right)=f(u) M_{E}(1)=f(u)
$$

e então

$$
\begin{aligned}
P(f)(u)=Q_{X}\left(Q_{E}(f)\right)(u) & =M_{X}\left(\left(Q_{E}(f)(u+x)-Q_{E}(f)(x)\right)_{x \in X}\right) \\
& =M_{X}\left(Q_{E}(f)(x)+Q_{E}(f)(u)-Q_{E}(f)(x)\right)_{x \in X} \\
& =Q_{E}(f)(u)=f(u),
\end{aligned}
$$

mostrando que $\left.P(f)\right|_{E}=\left.f\right|_{E}$. Resta provar que $P\left[\operatorname{Lip}_{0}(X)\right]=X^{*}$. Temos para cada $f \in$ $\operatorname{Lip}_{0}(X)$

$P(f)(x+y)=Q_{X}\left(Q_{E}(f)\right)(x+y)=Q_{X}\left(Q_{E}(f)\right)(x)+Q_{X}\left(Q_{E}(f)\right)(y)=P(f)(x)+P(f)(y)$.

Como

$$
Q_{X}(f)(-x)=-M_{X}\left((f(x+u)-f(u))_{u \in E}\right)=-Q_{X}(f)(x),
$$

segue que $P(f)(r x)=r P(f)(x)$ para todo $r \in \mathbb{Q}$. Se $\alpha \in \mathbb{R}$, tome uma sequência de racionais $\left(r_{n}\right)_{n \in \mathbb{N}}$ convergindo a $\alpha$. Então

$$
P(f)(\alpha x)=P(f)\left(\lim _{n \in \mathbb{N}} r_{n} x\right)=\lim _{n \in \mathbb{N}} P(f)\left(r_{n} x\right)=\lim _{n \in \mathbb{N}} r_{n} P(f)(x)=\alpha P(f)(x) .
$$

. Portanto $P(f)$ é linear para todo $f \in \operatorname{Lip}_{0}(X)$ e o resultado segue.

Teorema 3.5.13. Sejam $X$ e $Y$ espaços de Banach separáveis reflexivos e Lipschitz isomorfos. Então $X$ é isomorfo a um subespaço complementado de $Y$ e vice-versa. 
Demonstração. Seja $f: X \rightarrow Y$ um isomorfismo Lipschitz e $g=f^{-1}$. Podemos supor sem perda de generalidade que $f$ é Gâteaux diferenciável em 0 e que $f(0)=0$. Seja $S$ a derivada de $f$. Sabemos que $S$ será um isomorfismo de $X$ em um subespaço fechado de $Y$ (este tem $R N P$ por 3.2 .16 pois é reflexivo). Como $X$ é reflexivo, $B_{X}$ é compacto na topologia fraca. Fixado um ultrafiltro $\mathcal{U}$ não principal em $\mathbb{N}$, podemos definir $h: Y \rightarrow X$ por $h(y)=w-\lim _{n \in \mathcal{U}} n g(y / n)$, e esta função está bem definida pois

$$
\|n g(y / n)\|=\|n g(y / n)-n g(0 / n)\| \leq \operatorname{Lip}(g)\|y\| \text { para cada } n \in \mathbb{N},
$$

ou seja, a sequência $(n g(y / n))_{n \in \mathbb{N}}$ mora no conjunto $\operatorname{Lip}(g)\|y\| B_{X}$, que é compacto com respeito à topologia fraca por 2.3.34.

Temos que $h$ é lipschitziana pois vale para cada $n \in \mathbb{N}$ :

$$
\|n g(y / n)-n g(x / n)\| \leq n \operatorname{Lip}(g)\|x / n-y / n\|=\operatorname{Lip}(g)\|x-y\|
$$

e assim aplicamos 2.3.53. Além disso, temos que

$$
h(S(x))=\lim _{n \in \mathcal{U}} n g(S(x) / n)=\lim _{n \in \mathcal{U}} n g(f(x / n)-r(x / n))
$$

onde $r(x)=f(0+x)-f(0)-D_{f}(0)(x)=f(x)-D_{f}(0)(x)$. Mas para cada $x \in X$ e $n \in \mathbb{N}$, temos

$$
\|x-n g(f(x / n)-r(x / n))\|=\|n g(f(x / n))-n g(f(x / n)-r(x / n))\| \leq \operatorname{Lip}(g) n\|r(x / n)\| .
$$

Como $\lim _{n \in \mathbb{N}} n r(x / n)=0$, segue que

$$
h(S(x))=\lim _{n \in \mathcal{U}} n g(f(x / n)-r(x / n))=x \text { para todo } x \in X,
$$

$\operatorname{assim} h(S(x))=x$. Defina $V: X^{*} \rightarrow \operatorname{Lip}_{0}(Y)$ por $V\left(x^{*}\right)=x^{*} \circ h$ e $T: X^{*} \rightarrow Y^{*}$ por $T=P V$, onde $P: \operatorname{Lip}_{0}(Y) \rightarrow Y^{*}$ é a projeção dada pela Proposição 3.5.12. Veja que se $x^{*} \in X^{*}$, então

$$
V\left(x^{*}\right)(S(x))=x^{*}(h(S(x)))=x^{*}(x) .
$$

Logo, para cada $x^{*} \in X^{*}$ :

$$
T^{*}(S(x))\left(x^{*}\right)=S(x)\left(T\left(x^{*}\right)\right)=T\left(x^{*}\right)(S(x))=P\left(V\left(x^{*}\right)(S(x))\right)=x^{*}(x)
$$

donde segue que $T^{*} \circ S=I d_{X}$. Assim, $S(X)$ é um subespaço complementado em $Y$.

Sejam $X$ e $Y$ espaços de Banach tais que $X$ é isomorfo a um subespaço complementado de $Y$ e vice-versa. É desejável que possamos deduzir então que $X$ e $Y$ são isomorfos. O critério abaixo estabelece uma classe de espaços para os quais esse problema tem uma solução. 
Definição 3.5.14. Seja $X$ um espaço de Banach. Para cada $1 \leq p<\infty$, definimos o espaço $\ell_{p}(X)=\left\{\left(x_{n}\right)_{n \in \mathbb{N}} \in X^{\mathbb{N}}: \sum_{n \in \mathbb{N}}\left\|x_{n}\right\|^{p}<\infty\right\}$. Definimos também o espaço $c_{0}(X)=\left\{\left(x_{n}\right)_{n \in \mathbb{N}} \in\right.$ $\left.X^{\mathbb{N}}: \lim _{n \in \mathbb{N}}\left\|x_{n}\right\|=0\right\}$.

Observação 3.5.15. A função $\|\cdot\|_{p}: \ell_{p}(X) \rightarrow[0, \infty)$ dada por

$$
\left\|\left(x_{n}\right)_{n \in \mathbb{N}}\right\|_{p}=\left(\sum_{n \in \mathbb{N}}\left\|x_{n}\right\|^{p}\right)^{1 / p}=\left\|\left(\left\|x_{n}\right\|\right)_{n \in \mathbb{N}}\right\|_{\ell_{p}},
$$

é uma norma em $\ell_{p}(X)$. Analogamente, a função $\|\cdot\|_{\infty}: c_{0}(X) \rightarrow[0, \infty)$ dada por

$$
\left\|\left(x_{n}\right)_{n \in \mathbb{N}}\right\|_{\infty}=\sup _{n \in \mathbb{N}}\left\|x_{n}\right\|=\left\|\left(\left\|x_{n}\right\|\right)_{n \in \mathbb{N}}\right\|_{c_{0}},
$$

é uma norma em $c_{0}(X)$.

Teorema 3.5.16 (Técnica de Decomposição de Pełczyński). Sejam X e Y espaços de Banach tais que $X$ é isomorfo a um subespaço complementado de $Y$ e $Y$ é isomorfo a um subespaço complementado de X. Suponha que uma das propriedades abaixo seja verdadeira.

1. $X$ é isomorfo a $X^{2}$ e $Y$ é isomorfo a $Y^{2}$.

2. $X$ é isomorfo a $c_{0}(X)$ ou a um dos espaços $\ell_{p}(X)$, para $1 \leq p<\infty$.

Então $X$ é isomorfo a $Y$.

Demonstração. Ver [AK98] Teorema 2.2.3, página 34.

Lema 3.5.17. Sejam $1<p<\infty$ e $X=\ell_{p}$ ou $X=L_{p}$. Então $X$ é isomorfo a $\ell_{p}(X)$.

Demonstração. Suponha primeiro que $X=\ell_{p}$. Note que um elemento de $\ell_{p}(X)$ é da forma $\left(\left(x_{n, m}\right)_{n \in \mathbb{N}}\right)_{m \in \mathbb{N}}$, onde $\left(x_{n, m}\right)_{n \in \mathbb{N}} \in \ell_{p}$ para cada $m \in \mathbb{N}$ e tal que

$$
\left(\sum_{n \in \mathbb{N}} \sum_{m \in \mathbb{N}}\left|x_{n, m}\right|^{p}\right)^{1 / p}<\infty
$$

Seja $\varphi: \mathbb{N} \rightarrow \mathbb{N} \times \mathbb{N}$ uma bijeção. Definimos $T: \ell_{p}(X) \rightarrow \ell_{p}$ por

$$
T\left(\left(\left(x_{n, m}\right)_{n \in \mathbb{N}}\right)_{m \in \mathbb{N}}\right)=\left(x_{\varphi(n)}\right)_{n \in \mathbb{N}} \text { para cada }\left(\left(x_{n, m}\right)_{n \in \mathbb{N}}\right)_{m \in \mathbb{N}} \in \ell_{p}(X) .
$$


Veja que para cada $\left(\left(x_{n, m}\right)_{n \in \mathbb{N}}\right)_{m \in \mathbb{N}} \in \ell_{p}(X)$, temos

$$
\begin{aligned}
\left\|\left(\left(x_{n, m}\right)_{n \in \mathbb{N}}\right)_{m \in \mathbb{N}}\right\|_{p} & =\left(\sum_{n \in \mathbb{N}} \sum_{m \in \mathbb{N}}\left|x_{n, m}\right|^{p}\right)^{1 / p} \\
& =\left(\sum_{n, m \in \mathbb{N}}\left|x_{n, m}\right|^{p}\right)^{1 / p} \\
& =\left(\sum_{k \in \mathbb{N}}\left|x_{\varphi(k)}\right|^{p}\right)^{1 / p} \\
& =\left\|T\left(\left(\left(x_{n, m}\right)_{n \in \mathbb{N}}\right)_{m \in \mathbb{N}}\right)\right\|_{\ell_{p}} .
\end{aligned}
$$

Logo $T$ é uma isometria. É também sobrejetora pois se $\left(y_{n}\right)_{n} \in \ell_{p}$, tomamos $x_{n, m}=y_{\varphi^{-1}(n, m)}$ para todos $n, m \in \mathbb{N}$ e então

$$
T\left(\left(\left(x_{n, m}\right)_{n \in \mathbb{N}}\right)_{m \in \mathbb{N}}\right)=\left(x_{\varphi(n)}\right)_{n \in \mathbb{N}}=\left(y_{\varphi^{-1}(\varphi(n))}\right)_{n \in \mathbb{N}}=\left(y_{n}\right)_{n \in \mathbb{N}}
$$

Suponha agora que $X=L_{p}$. Note que um elemento de $\ell_{p}(X)$ é da forma $\left(f_{n}\right)_{n \in \mathbb{N}}$, onde $f_{n} \in L_{p}$ para cada $n \in \mathbb{N}$ e

$$
\left(\sum_{n \in \mathbb{N}} \int_{0}^{1}\left|f_{n}(t)\right|^{p} d t\right)^{1 / p}<\infty
$$

Definimos $T: \ell_{p}\left(L_{p}\right) \rightarrow L_{p}$ por

$$
T\left(\left(f_{n}\right)_{n \in \mathbb{N}}\right)(t)=\left\{\begin{array}{ll}
2^{n+1} f_{n}\left(2^{n+1} t-1\right) & \frac{1}{2^{n+1}}<t \leq \frac{1}{2^{n}} \\
0 & t=0
\end{array} \text { para cada }\left(f_{n}\right)_{n \in \mathbb{N}} \in \ell_{p}(X) .\right.
$$

Temos então para cada $\left(f_{n}\right)_{n \in \mathbb{N}} \in \ell_{p}(X)$ que

$$
\begin{aligned}
\left\|T\left(\left(f_{n}\right)_{n \in \mathbb{N}}\right)\right\| & =\left(\int_{0}^{1}\left|T\left(\left(f_{n}\right)_{n \in \mathbb{N}}\right)(t)\right|^{p} d t\right)^{1 / p} \\
& =\left(\sum_{n \in \mathbb{N}} \int_{\frac{1}{2^{n+1}}}^{\frac{1}{2^{n}}} 2^{n+1}\left|f_{n}\left(2^{n+1} t-1\right)\right|^{p} d t\right)^{1 / p} \\
& =\left(\sum_{n \in \mathbb{N}} \int_{0}^{1}\left|f_{n}(t)\right|^{p} d t\right)^{1 / p} \\
& =\left\|\left(f_{n}\right)_{n \in \mathbb{N}}\right\|_{p} .
\end{aligned}
$$

Logo $T$ é uma isometria. É também sobrejetora pois se $f \in L_{p}$, definimos $f_{n}(t)=\frac{1}{2^{n+1}} f\left(\frac{t+1}{2^{n+1}}\right)$. 
Então

$$
\begin{aligned}
T\left(\left(f_{n}\right)_{n \in \mathbb{N}}\right)(t) & = \begin{cases}2^{n+1} f_{n}\left(2^{n+1} t-1\right) & \frac{1}{2^{n+1}}<t \leq \frac{1}{2^{n}} \\
0 & t=0\end{cases} \\
& = \begin{cases}f(t) & \frac{1}{2^{n+1}}<t \leq \frac{1}{2^{n}} \\
0 & t=0\end{cases} \\
& =f(t) .
\end{aligned}
$$

Teorema 3.5.18. Sejam $1<p<\infty$ e $X=\ell_{p}$ ou $X=L_{p}$. Suponha que $Y$ seja um espaço de Banach Lipschitz isomorfo a $X$. Então $Y$ é isomorfo a $X$.

Demonstração. Seja $f: Y \rightarrow X$ um isomorfismo Lipschitz. Como $X$ é reflexivo, $X$ tem $\mathrm{RNP}$ e $\log$ o $Y$ é isomorfo a um subespaço de $X$, assim $Y$ também é reflexivo. Pelo Teorema 3.5.13, $X$ é isomorfo a um subespaço complementado de $Y$ e vice-versa. Mas pelo Lema 3.5.17, $X$ e $Y$ satisfazem a decomposição de Pełczyński. Segue então que $X$ e $Y$ são isomorfos. 


\section{Capítulo 4}

\section{HOMEOMORFISMOS UNIFORMES ENTRE ESPAÇOS DE BANACH}

Neste capítulo vamos focar nos homeomorfismos uniformes entre espaços de Banach. Diferente dos isomorfismos Lipschitz, não temos técnicas de diferenciabilidade em mãos. Entretanto, vamos introduzir os conceitos de pontos médio aproximados e de grafos métricos, e estes vão quantificar algumas propriedades entre espaços de Banach uniformemente homeomorfos. Nosso objetivo principal é mostrar o clássico resultado de [JLS96]: se $1<p<\infty$ e $X$ é uniformemente homeomorfo a $\ell_{p}$, então $X$ é isomorfo a $\ell_{p}$. Após isso, vamos reaproveitar as técnicas desenvolvidas para provar resultados análogos de [JLS96] e [KR08] para espaços da forma $\ell_{p} \oplus \ell_{q}$.

\subsection{Definições Básicas}

Primeiramente, vamos definir algumas classes de funções. Indicamos [Kal08] como referência desta seção.

Definição 4.1.1. Sejam $\left(M_{1}, d_{1}\right)$ e $\left(M_{2}, d_{2}\right)$ espaços métricos e $f: M_{1} \rightarrow M_{2}$ uma função. Definimos o módulo de continuidade de $f$ por

$$
\omega_{f}(t)=\sup \left\{d_{2}(f(x), f(y)): x, y \in M_{1} \text { e } d_{1}(x, y) \leq t\right\} \text { para cada } t>0 .
$$

Definição 4.1.2. Sejam $M_{1}, M_{2}$ espaços métricos e $f: M_{1} \rightarrow M_{2}$ uma função. Dizemos que $f$ é uniformemete continua se $\lim _{t \rightarrow 0^{+}} \omega_{f}(t)=0$.

Definição 4.1.3. Sejam $M_{1}$ e $M_{2}$ espaços métricos. Dizemos que $M_{1}$ e $M_{2}$ são uniformemente homeomorfos se existe uma função invertivel $f: M_{1} \rightarrow M_{2}$ uniformemente continua tal que sua inversa $f^{-1}: M_{2} \rightarrow M_{1}$ é uniformemente contínua. Neste caso, dizemos que $f$ é um homeomorfismo uniforme. 
Vamos definir agora o que é uma função grosseiramente contínua. Estas funções preservam a estrutura grosseira de um espaço métrico e basicamente são funções que enviam conjuntos limitados do domínio em conjuntos limitados do contradomínio.

Definição 4.1.4. Sejam $M_{1}, M_{2}$ espaços métricos e $f: M_{1} \rightarrow M_{2}$ uma função. Dizemos que $f$ é grosseiramente contínua, ou coarsely contínua, se $\omega_{f}(t)<\infty$ para cada $t>0$.

Ou seja, funções grosseiramente contínuas enviam conjuntos limitados do domínio em conjuntos limitados do codomínio. Um conceito um pouco mais refinado de função grosseiramente contínua é o conceito de função grosseira Lipschitz contínua.

Definição 4.1.5. Sejam $M_{1}, M_{2}$ espaços métricos e $f: M_{1} \rightarrow M_{2}$ uma função. Dizemos que $f$ é grosseira Lipschitz contínua (CLC), ou coarse Lipschitz contínua, se $\limsup _{t \rightarrow \infty} \omega_{f}(t) / t<$ $\infty$. Neste caso, vamos denotar $\limsup _{t \rightarrow \infty} \omega_{f}(t) / t$ por $\operatorname{Lip}_{\infty}(f)$.

Definição 4.1.6. Sejam $\left(M_{1}, d_{1}\right),\left(M_{2}, d_{2}\right)$ espaços métricos e $f: M_{1} \rightarrow M_{2}$ uma função. Definimos para cada $t>0$

$$
K_{f}(t)=\sup \left\{\frac{d_{2}(f(x), f(y))}{\max \left\{t, d_{1}(x, y)\right\}}: x, y \in M_{1} \text { e } x \neq y\right\}
$$

Observação 4.1.7. Note que para $t \geq$ s e para cada $x, y \in M_{1}$ distintos, temos $\max \left\{t, d_{1}(x, y)\right\} \geq$ $\max \left\{s, d_{1}(x, y)\right\}$ e assim

$$
\frac{d_{2}(f(x), f(y))}{\max \left\{t, d_{1}(x, y)\right\}} \leq \frac{d_{2}(f(x), f(y))}{\max \left\{s, d_{1}(x, y)\right\}}
$$

portanto $K_{f}(t) \leq K_{f}(s)$.

Lema 4.1.8. Sejam $M_{1}, M_{2}$ espaços métricos e $f: M_{1} \rightarrow M_{2}$ uma função. Então $K_{f}(t)=$ $\sup _{s \geq t} \omega_{f}(s) / s$.

Demonstração. Dados $t>0$ e $x, y \in M_{1}$ distintos, então ou $d_{1}(x, y) \leq t$ e neste caso

$$
\frac{d_{2}(f(x), f(y))}{\max \left\{t, d_{1}(x, y)\right\}}=\frac{d_{2}(f(x), f(y))}{t} \leq \omega_{f}(t) / t \leq \sup _{s \geq t} \omega_{f}(s) / s,
$$

ou $d_{1}(x, y) \geq t$ e fazendo $s_{0}=d_{1}(x, y)$, temos

$$
\frac{d_{2}(f(x), f(y))}{\max \left\{t, d_{1}(x, y)\right\}}=\frac{d_{2}(f(x), f(y))}{s_{0}} \leq \omega_{f}\left(s_{0}\right) / s_{0} \leq \sup _{s \geq t} \omega_{f}(s) / s .
$$

Segue então que $K_{f}(t) \leq \sup _{s \geq t} \omega_{f}(s) / s$. Por outro lado, seja $s \geq t$ e $x, y \in M_{1}$ tais que $d_{1}(x, y) \leq s$. Então $\max \left\{t, d_{1}(x, y)\right\} \leq s$ e assim

$$
\frac{d_{2}(f(x), f(y))}{s} \leq \frac{d_{2}(f(x), f(y))}{\max \left\{t, d_{1}(x, y)\right\}} \leq K_{f}(t),
$$


e segue que $\omega_{f}(s) / s \leq K_{f}(t)$. Pela arbitrariedade de $s \geq t$, concluimos que $\sup _{s \geq t} \omega_{f}(s) / s \leq$ $K_{f}(t)$.

Proposição 4.1.9. Sejam $M_{1}, M_{2}$ espaços métricos e $f: M_{1} \rightarrow M_{2}$ uma função. Então $f$ é CLC se e somente se $K_{f}(t)<\infty$ para todo $t>0$.

Demonstração. Suponha que $\limsup _{t \rightarrow \infty} \omega_{f}(t) / t<\infty$. Então existe um $t_{0}>0$ tal que $\sup _{s \geq t_{0}} \omega_{f}(s) / s<$ $\infty$. Em particular

$$
K_{f}(t) \leq K_{f}\left(t_{0}\right)=\sup _{s \geq t_{0}} \omega_{f}(s) / s<\infty \text { para cada } t \geq t_{0}
$$

Se $0<t<t_{0}$, temos

$$
K_{f}(t)=\sup _{s \geq t} \omega_{f}(s) / s=\max \left\{\sup _{s \geq t_{0}} \omega_{f}(s) / s, \sup _{t \leq s \leq t_{0}} \omega_{f}(s) / s\right\} \leq \max \left\{K_{f}\left(t_{0}\right), \omega_{f}\left(t_{0}\right) / t\right\}<\infty .
$$

Por fim, se $K_{f}(t)<\infty$ para todo $t>0$, segue que $\inf _{t>0} K_{f}(t)<\infty$ e

$$
\limsup _{t \rightarrow \infty} \omega_{f}(t) / t=\inf _{t>0} \sup _{s \geq t} \omega_{f}(s) / s=\inf _{t>0} K_{f}(t)<\infty
$$

Por conta da proposição acima, as funções CLC também são chamadas de funções Lipschitz para grandes distâncias pois se $f: M_{1} \rightarrow M_{2}$ é CLC, para cada $t>0$, temos que

$$
d_{2}(f(x), f(y)) \leq K_{f}(t) d_{1}(x, y) \text { para cada } x, y \in M_{1} \operatorname{com} d_{1}(x, y) \geq t
$$

A seguir, vamos definir quando dois espaços são grosseiramente homeomorfos. Diferente do usual, não é necessário que a função seja uma bijeção.

Definição 4.1.10. Dizemos que $M_{1}$ e $M_{2}$ são grosseiramente (grosseiro Lipschitz) homeomorfos se existem funções $f: M_{1} \rightarrow M_{2}$ e $g: M_{2} \rightarrow M_{1}$ grosseiramente (grosseiro Lipschitz) continuas tais que

$$
\sup _{x \in M_{1}} d(x, g \circ f(x))<\infty e \sup _{y \in M_{2}} d(y, f \circ g(y))<\infty
$$

Exemplo 4.1.11. $\mathbb{R}$ munido da métrica usual e $(\mathbb{R}, \rho)$, onde $\rho(x, y)=\min (1,|x-y|)$, são uniformemente homeomorfos mas não são grosseiramente homeomorfos. De fato, se $f:(\mathbb{R},|\cdot|) \rightarrow(\mathbb{R}, \rho)$ e $g:(\mathbb{R}, \rho) \rightarrow(\mathbb{R},|\cdot|)$ são grosseiramente contínuas então $g[f[\mathbb{R}]]$ é limitado, e logo $\{|x-g \circ f(x)|: x \in \mathbb{R}\}$ é ilimitado.

Exemplo 4.1.12. $\mathbb{R}$ munido da métrica usual e $(\mathbb{R}, \sigma)$, onde $\sigma(x, y)=|x-y|+1$ se $x \neq y$ e $\sigma(x, x)=0$ para cada $x \in \mathbb{R}$, são grosseiramente homeomorfos mas não são uniformemente 
isomorfos. De fato, não existe uma função uniformemente continua $f:(\mathbb{R},|\cdot|) \rightarrow(\mathbb{R}, \sigma)$. Além disso, a função identidade $I d:(\mathbb{R},|\cdot|) \rightarrow(\mathbb{R}, \sigma)$ é grosseiramente contínua pois $\omega_{I d}(t) \leq t+1$ para cada $0<t<\infty$. A função $I d_{\sigma}:(\mathbb{R}, \sigma) \rightarrow(\mathbb{R},|\cdot|)$ também é grosseiramente continua pois $\omega_{I d_{\sigma}}(t) \leq t-1$ para cada $1<t<\infty$ e $\omega_{I d_{\sigma}}(t)=0$ para $0<t \leq 1$.

Para certos espaços métricos, os conceitos de funções grosseiramente contínuas e Lipschitz para grandes distâncias são equivalentes. Este será o caso, por exemplo, de espaços normados.

Definição 4.1.13. Dizemos que um espaço métrico $M$ é metricamente convexo se para cada $x, y \in M$ e $\lambda \in(0,1)$, existe $z \in M$ tal que $d(x, z)=\lambda d(x, y)$ e $d(y, z)=(1-\lambda) d(x, y)$.

Lema 4.1.14. Se $M_{1}$ é um espaço métrico convexo, $M_{2}$ é um espaço métrico e $f: M_{1} \rightarrow M_{2}$ então $\omega_{f}$ é uma função subaditiva, isto é, $\omega_{f}(s+t) \leq \omega_{f}(s)+\omega_{f}(t)$ para todos $s, t>0$.

Demonstração. Sejam $0<s<t$. Pelo teorema do valor intermediário, existe $\beta \in(0,1)$ tal que $s=\frac{\beta}{1-\beta} t$. Repare que

$$
\beta(s+t)=\beta\left(s+\frac{1-\beta}{\beta} s\right)=s,
$$

e analogamente temos que $(1-\beta)(s+t)=t$. Assim, se $d(x, y) \leq s+t$, existe $z \in M$ tal que $d(x, z) \leq \beta(s+t)=s$ e $d(y, z) \leq(1-\beta)(s+t)=t$, e então

$$
d(f(x), f(y)) \leq d(f(x), f(z))+d(f(z), f(y)) \leq \omega_{f}(s)+\omega_{f}(t) .
$$

Segue portanto que $\omega_{f}(s+t) \leq \omega_{f}(s)+\omega_{f}(t)$. No caso em que $s=t$, para cada $x, y \in M_{1}$ com $d(x, y) \leq 2 t$, tome $z \in M_{1}$ tal que $d(x, z), d(z, y)=\frac{1}{2} d(x, y) \leq t$. Então

$$
d(f(x), f(y)) \leq d(f(x), f(z))+d(f(z), f(x)) \leq 2 \omega_{f}(t)
$$

e portanto $\omega_{f}(2 t) \leq 2 \omega_{f}(t)$

Lema 4.1.15. Seja $M_{1}$ metricamente convexo e $f: M_{1} \rightarrow M_{2}$. São equivalentes:

1. f é grosseiramente contínua.

2. Existe $0<t<\infty$ tal que $\omega_{f}(t)<\infty$.

3. fé $C L C$.

Em particular, se $f$ é uniformemente contínua, $f$ é grosseiramente contínua.

Demonstração. 2) $\Rightarrow 1$ ) Seja $t>0$ tal que $\omega_{f}(t)<\infty$. Veja que se $s<t$, então $\omega_{f}(s) \leq$ $\omega_{f}(t)<\infty$. Se $n \in \mathbb{N}$, temos que $\omega_{f}(n t) \leq n \omega_{f}(t)$ pois $\omega_{f}$ é subaditiva e como para cada $0<s<\infty$ existe $n$ suficientemente grande tal que $s<n t$, temos

$$
\omega_{f}(s) \leq \omega_{f}(n t) \leq n \omega_{f}(t)<\infty .
$$


Fica claro então que 1) e 2) são equivalentes.

Resta provar que 2) $\Rightarrow 3$ ) pois 3) $\Rightarrow 2$ ) é direto. Dado $s \geq t$, tome $n \in \mathbb{N}$ tal que $(n-1) t \leq$ $s<n t$. Então

$$
\frac{1}{s} \omega_{f}(s) \leq \frac{n \omega_{f}(t)}{s} \leq \frac{n}{(n-1) t} \omega_{f}(t) \leq \frac{2}{t} \omega_{f}(t)
$$

e segue então que

$$
\sup _{s \geq t} \omega_{f}(s) / s \leq \frac{2}{t} \omega_{f}(t)<\infty
$$

Portanto $\limsup _{t \rightarrow \infty} \omega_{f}(t) / t<\infty$.

Exemplo 4.1.16. A função $f: \mathbb{R} \rightarrow \mathbb{Z}$, onde $f(x)$ é a parte inteira de $x$ para cada $x \in \mathbb{R}$, é uma função $C L C$. De fato, veja que $|f(x)-f(y)| \leq|x-y|+2$ para todos $x, y \in \mathbb{R}$, logo $\omega_{f}(t) \leq t+2<\infty$ para todo $t>0$. Segue então que $f$ é CLC pois $\mathbb{R}$ é convexo.

No caso particular dos espaços de Banach, temos então a seguinte equivalência.

Proposição 4.1.17. Sejam X e $Y$ espaços de Banach. São equivalentes:

1. X e Y são grosseiramente homeomorfos.

2. X e Y são grosseiro Lipschitz homeomorfos.

Em particular, se $X$ e $Y$ são uniformemente homeomorfos, então X e $Y$ também são grosseiramente homeomorfos.

Uma conexão entre homeomorfismos uniformes (ou no caso mais geral de homeomorfismos grosseiros) e isomorfismos Lipschitz é dada pelo resultado de Heinrich e Mankiewicz (veja [HM82]) abaixo.

Teorema 4.1.18 (Heinrich-Mankiewicz). Sejam X e $Y$ espaços de Banach grosseiramente homeomorfos. Então $X_{\mathcal{U}}$ e $Y_{\mathcal{U}}$ são Lipschitz isomorfos para qualquer que seja o ultrafiltro não principal $\mathcal{U}$ em $\mathbb{N}$.

Demonstração. Seja $f: X \rightarrow Y$ grosseiramente contínua dada pela definição 4.1.10. Defina $F: X_{\mathcal{U}} \rightarrow Y_{\mathcal{U}}$ por $F\left(\overline{\left(x_{n}\right)_{n \in \mathbb{N}}}\right)=\overline{\left(f\left(n x_{n}\right) / n\right)_{n \in \mathbb{N}}}$. Veja que $F$ está bem definida pois se $\left(z_{n}\right)_{n \in \mathbb{N}} \in \overline{\left(x_{n}\right)_{n \in \mathbb{N}}}$, por 2.3.61 temos $\lim _{n \in \mathcal{U}}\left\|x_{n}-z_{n}\right\|=0$ e para cada $n \in \mathbb{N}$

$$
\begin{aligned}
\left\|f\left(n x_{n}\right)-f\left(n z_{n}\right)\right\| / n & \leq K_{f}(1) \max \left\{n\left\|x_{n}-z_{n}\right\|, 1\right\} / n \\
& =K_{f}(1) \max \left\{\left\|x_{n}-z_{n}\right\|, 1 / n\right\} .
\end{aligned}
$$

Mas para cada $\epsilon>0$ temos que $\left\{n \in \mathbb{N}: \max \left\{\left\|x_{n}-z_{n}\right\|, 1 / n\right\}<\epsilon / K_{f}(1)\right\} \in \mathcal{U}$. Mas este conjunto está contido em $\left\{n \in \mathbb{N}:\left\|\frac{f\left(n x_{n}\right)}{n}-\frac{f\left(n z_{n}\right)}{n}\right\|<\epsilon\right\}$. De fato, se $n \in \mathbb{N}$ é tal que $\max \left\{\left\|x_{n}-z_{n}\right\|, 1 / n\right\}<\epsilon / K_{f}(1)$, então

$$
\left\|f\left(n x_{n}\right)-f\left(n z_{n}\right)\right\| / n \leq K_{f}(1) \max \left\{\left\|x_{n}-z_{n}\right\|, 1 / n\right\}<\epsilon .
$$


Segue que $\left\{n \in \mathbb{N}:\left\|\frac{f\left(n x_{n}\right)}{n}-\frac{f\left(n z_{n}\right)}{n}\right\|<\epsilon\right\} \in \mathcal{U}$ e então, como $\epsilon>0$ é arbitrário, temos que $\lim _{n \in \mathcal{U}}\left\|\frac{f\left(n x_{n}\right)}{n}-\frac{f\left(n z_{n}\right)}{n}\right\|=0$.

Vamos mostrar que $F$ é Lipschitziana. Como $f$ é CLC, por 4.1 .9 existe $K=K_{f}(1)$ tal que $\|x-y\| \geq 1$ implica:

$$
\|f(x)-f(y)\| \leq K\|x-y\|
$$

Considere $\bar{x}, \bar{y} \in X_{\mathcal{U}}$ distintos e $x=\left(x_{n}\right) \in \bar{x}, y=\left(y_{n}\right) \in \bar{y}$ representantes. Seja $n_{0} \in \mathbb{N}$ tal que $\alpha=\|\bar{x}-\bar{y}\|_{X_{\mathcal{U}}}>1 / n_{0}$.

Afirmamos que $D=\left\{n \in \mathbb{N}:\left\|x_{n}-y_{n}\right\| \geq 1 / n_{0}\right\} \in \mathcal{U}$. De fato, seja $\delta>0$ tal que $|r-\alpha|<\delta$ implica $r \geq 1 / n_{0}$. Mas por 2.3.61, $\alpha=\lim _{n \in \mathcal{U}}\left\|x_{n}-y_{n}\right\|$ e temos que $\left\{m \in \mathbb{N}:\left|\left\|x_{m}-y_{m}\right\|-\alpha\right|<\right.$ $\delta\} \in \mathcal{U}$ e pela escolha de $\delta$, este conjunto está contido em $D$. Assim temos que $D \in \mathcal{U}$. Tome $D_{0}=D \cap\left\{m \in \mathbb{N}: m \geq n_{0}\right\}$. Como $\mathcal{U}$ é não principal, por 2.3.47 e 2.3.49 temos que $\left\{m \in \mathbb{N}: m \geq n_{0}\right\} \in \mathcal{U}$ e assim $D_{0} \in U$. Logo, se $n \in D_{0}$, temos $\left\|x_{n}-y_{n}\right\| \geq 1 / n_{0} \geq 1 / n$ e

$$
\left\|f\left(n x_{n}\right)-f\left(n y_{n}\right)\right\| \leq K\left\|n x_{n}-n y_{n}\right\|
$$

e assim, pelo Lema 2.3.53, obtemos

$$
\|F(\bar{x})-F(\bar{y})\|_{Y_{\mathcal{U}}} \leq K\|\bar{x}-\bar{y}\|_{X_{\mathcal{U}}}
$$

Seja agora $g: Y \rightarrow X$ grosseiramente contínua tal que

$$
\sup _{x \in X} d(x, g \circ f(x))<\infty \text { e } \sup _{y \in Y} d(y, f \circ g(y))<\infty .
$$

Podemos definir a função Lipschitziana $G: Y_{\mathcal{U}} \rightarrow X_{\mathcal{U}}$ por $G\left(\left(y_{n}\right)_{n \in \mathbb{N}}\right)=\overline{\left(g\left(n y_{n}\right) / n\right)_{n \in \mathbb{N}}}$. Seja $\overline{\left(y_{n}\right)_{n \in \mathbb{N}}} \in Y_{\mathcal{U}}$. Como $\sup _{y \in Y}\|y-f \circ g(y)\|=M$ para algum $M>0$, temos $\left\|n y_{n}-f\left(g\left(n y_{n}\right)\right)\right\| \leq M$ para cada $n \in \mathbb{N}$ e assim $\left\|y_{n}-f\left(g\left(n y_{n}\right)\right) / n\right\| \leq M / n$, mas então

$$
\lim _{n \in \mathcal{U}}\left\|f\left(g\left(n y_{n}\right)\right) / n-y_{n}\right\|=0
$$

isto é, $F \circ G\left(\overline{\left(y_{n}\right)_{n \in \mathbb{N}}}\right)=\overline{\left(y_{n}\right)_{n \in \mathbb{N}}}$, ou seja, $F \circ G=I d_{Y_{\mathcal{U}}}$. Analogamente provamos que $G \circ F=I d_{X_{\mathcal{U}}}$.

Em particular, podemos deduzir, junto aos resultados que vimos no capítulo 3, os seguintes teoremas. Este primeiro foi provado inicialmente por [Enf70].

Teorema 4.1.19. (Enflo) Seja X um espaço de Banach separável grosseiramente homeomorfo a um espaço de Hilbert. Então X é isomorfo a um espaço de Hilbert.

Demonstração. Sejam $H$ um espaço de Hilbert e $f: X \rightarrow H$ tal que $f$ é uma função grosseiramente contínua dada pela definição 4.1.10. Veja que a lei do paralelogramo vale em 
$H_{\mathcal{U}}$. De fato, dados $\bar{x}, \bar{y} \in H_{\mathcal{U}}$ e representantes $x=\left(x_{n}\right)_{n \in \mathbb{N}} \in \bar{x}, y=\left(y_{n}\right)_{n \in \mathbb{N}} \in \bar{y}$, temos

$$
\begin{aligned}
2\|x\|_{H_{\mathcal{U}}}^{2}+2\|y\|_{H_{\mathcal{U}}}^{2}=\lim _{n \in \mathcal{U}}\left(2\left\|x_{n}\right\|^{2}+2\left\|y_{n}\right\|^{2}\right) & =\lim _{n \in \mathcal{U}}\left(\left\|x_{n}+y_{n}\right\|^{2}+\left\|x_{n}-y_{n}\right\|^{2}\right) \\
& =\|x+y\|_{H_{\mathcal{U}}}^{2}+\|x-y\|_{H_{\mathcal{U}}}^{2} .
\end{aligned}
$$

Assim $H_{\mathcal{U}}$ também é um espaço de Hilbert. Seja $F: X_{\mathcal{U}} \rightarrow H_{\mathcal{U}}$ o isomorfismo Lipschitz construído como no Teorema 4.1.18. A função $\rho: X \rightarrow X_{\mathcal{U}}$ dada por $\rho(x)=\overline{\left(x_{n}\right)_{n \in \mathbb{N}}}$, onde $x_{n}=x$ para cada $n \in \mathbb{N}$, é uma isometria linear e logo $G=F \circ \rho$ é um mergulho Lipschitz de $X$ em $G(X) \subset H_{\mathcal{U}}$. Como $[G(X)]$ é um subespaço fechado separável de $H_{\mathcal{U}}$ (em particular, $[G(X)]$ possui RNP pois é Hilbert), podemos aplicar o Teorema 3.3.15 para garantir um mergulho linear de $X$ em $[G(X)]$ e portanto $X$ é um espaço de Hilbert.

O resultado abaixo será dado através das técnicas que estudamos e que foram desenvolvidas por [HM82], mas foi dado originalmente por [Rib76].

Teorema 4.1.20. (Ribe) Sejam X e $Y$ espaços de Banach separáveis. Suponha que X seja grosseiramente homeomorfo a $Y$. Então $X$ pode ser finitamente representável em $Y$ e viceversa.

Demonstração. Seja $G: X \rightarrow Y_{\mathcal{U}}$ um mergulho Lipschitz como construímos em 4.1.19. Então existe um mergulho linear $T: X \rightarrow Y_{\mathcal{U}}^{* *}$ pelo Corolário 3.4.10. O Princípio da Reflexividade Local 2.3.74 nos garante que $Y_{\mathcal{U}}^{* *}$ é finitamente representável em $Y_{\mathcal{U}}$, que por sua vez é finitamente representável em $Y$ pela Proposição 2.3.73. Logo $X$ é finitamente representável em $Y$.

Corolário 4.1.21. Sejam $X$ e $Y$ espaços de Banach. Suponha que $X$ seja grosseiramente homeomorfo a $Y$. Suponha também que $Y$ tem tipo $p$ e cotipo q para $1 \leq p \leq 2$ e $2 \leq q \leq \infty$. Então $X$ tem tipo $p$ e cotipo $q$ com as mesmas constantes de $Y$;

Demonstração. Segue do teorema de Ribe que $X$ é finitamente representável em $Y$, e o resultado segue pela Proposição 2.3.79.

\subsection{Pontos médio aproximados}

O conceito de pontos médio aproximados é inspirado na demonstração do teorema de Mazur-Ulam (veja A.0.3), onde é mostrado que pontos médio são preservados por isometrias. Vamos mostrar que os pontos médio aproximados são preservados por certas funções entre espaços de Banach, em particular por homeomorfismos uniformes. Assim, poderemos encontrar algumas estimativas sobre homeomorfismos uniformes que serão importantes nas seções seguintes.

Definição 4.2.1. Sejam $X$ um espaço normado, $x, y \in X$ e $\delta>0$. Definimos

$$
\operatorname{Mid}(x, y, \delta)=\{z \in X: \max (\|x-z\|,\|y-z\|) \leq(1+\delta)\|x-y\| / 2\}
$$


Chamamos os elementos de $\operatorname{Mid}(x, y, \delta)$ de pontos médio aproximados entre $x$ e $y$.

Definição 4.2.2. Sejam $M_{1}, M_{2}$ espaços métricos e $f: M_{1} \rightarrow M_{2}$ uma função. Definimos

$$
R_{f}(s)=\sup \left\{\frac{d(f(x), f(y))}{d(x, y)}: d(x, y) \geq s\right\}
$$

Lema 4.2.3. Sejam $X$ espaço normado, $M$ espaço métrico e $f: X \rightarrow M C L C$. Então $\operatorname{Lip}_{\infty}(f)=\inf _{s>0} R_{f}(s)$.

Demonstração. Temos por definição e pelo Lema 4.1.8 que:

$$
\begin{aligned}
\operatorname{Lip}_{\infty}(f) & =\inf _{s>0} \sup _{t \geq s} \omega_{f}(t) / t \\
& =\inf _{s>0} K_{f}(s) \\
& =\inf _{s>0} \sup _{t \geq s}\left\{\left\{\frac{d(f(x), f(y))}{\|x-y\|}:\|x-y\| \geq s\right\} \cup\left\{\frac{d(f(x), f(y))}{s}:\|x-y\| \leq s\right\}\right\} \\
& =\inf _{s>0} \max \left\{R_{f}(s), \omega_{f}(s) / s\right\} \\
& \geq \inf _{s>0} R_{f}(s) .
\end{aligned}
$$

Suponha por contradição que $\operatorname{Lip}_{\infty}(f)>\inf _{s>0} R_{f}(s)$. Então existe $t_{0}>0$ tal que

$$
R_{f}\left(t_{0}\right)<\limsup _{t \rightarrow \infty} \omega_{f}(t) / t
$$

Em particular, para cada $n>1$, temos $R_{f}\left(t_{0}\right)<\sup _{t \geq n t_{0}} \omega_{f}(t) / t$ e assim, para cada $n>1$ existe $t_{n} \geq n t_{0}$ tal que $R_{f}\left(t_{0}\right)<\omega_{f}\left(t_{n}\right) / t_{n}$. Logo, para cada $n>1$ existem $x_{n}, y_{n} \in X$ tais que $\left\|x_{n}-y_{n}\right\| \leq t_{n} \mathrm{e}$

$$
R_{f}\left(t_{0}\right)<d\left(f\left(x_{n}\right), f\left(y_{n}\right)\right) / t_{n}
$$

Vamos separar o final da demonstração em dois casos. Suponha primeiramente que exista $n>1$ tal que $\left\|x_{n}-y_{n}\right\| \geq n t_{0} \geq 2 t_{0}$. Tome $z=\left(x_{n}+y_{n}\right) / 2$ e note que

$$
t_{0} \leq \min \left\{\left\|z-x_{n}\right\|,\left\|z-y_{n}\right\|\right\} \leq \max \left\{\left\|z-x_{n}\right\|,\left\|z-y_{n}\right\|\right\} \leq t_{n} / 2 .
$$

Mas então teremos que

$$
\frac{d\left(f(z), f\left(x_{n}\right)\right)}{t_{n} / 2} \leq \frac{d\left(f(z), f\left(x_{n}\right)\right)}{\left\|z-x_{n}\right\|} \leq R_{f}\left(t_{0}\right)<d\left(f\left(x_{n}\right), f\left(y_{n}\right)\right) / t_{n}
$$

e analogamente $\frac{d\left(f(z), f\left(y_{n}\right)\right)}{t_{n} / 2}<d\left(f\left(x_{n}\right), f\left(y_{n}\right)\right) / t_{n}$. Isso contradiz a desigualdade triangular em $M$ pois obtemos

$$
d\left(f(z), f\left(x_{n}\right)\right)+d\left(f(z), f\left(y_{n}\right)\right)<2 d\left(f\left(x_{n}\right), f\left(y_{n}\right)\right) / 2=d\left(f\left(x_{n}\right), f\left(y_{n}\right)\right) .
$$

Suponha por fim que para cada $n>1,\left\|x_{n}-y_{n}\right\|<n t_{0}$. Pela escolha de $x_{n}$ e $y_{n}$, temos 
que $R_{f}\left(t_{0}\right)<\omega_{f}\left(n t_{0}\right) / n t_{0}$ para cada $n>1$. Vamos mostrar que para cada $n>1$ temos $\omega_{f}\left(n t_{0}\right)=\omega_{f}\left(t_{0}\right)$. De fato, caso houvessem $x, y \in X$ tais que $t_{0} \leq\|x-y\| \leq n t_{0}$ e $R_{f}\left(t_{0}\right)<$ $d(f(x), f(y)) / n t_{0}$, então

$$
d(f(x), f(y)) / n t_{0} \leq d(f(x), f(y)) /\|x-y\| \leq R_{f}\left(t_{0}\right)<d(f(x), f(y)) / n t_{0},
$$

uma contradição. Portanto o valor $\omega_{f}\left(n t_{0}\right)$ é atingido no conjunto $\left\{\|x-y\|:\|x-y\| \leq t_{0}\right\}$, isto é, $\omega_{f}\left(n t_{0}\right)=\omega_{f}\left(t_{0}\right)$ para cada $n>1$. Porém isto implica que

$$
\operatorname{Lip}_{\infty}(f)=\limsup _{t \rightarrow \infty} \omega_{f}(t) / t=\limsup _{n \in \mathbb{N}} \omega_{f}\left(n t_{0}\right) /\left(n t_{0}\right)=\lim _{n \in \mathbb{N}} \omega_{f}\left(t_{0}\right) /\left(n t_{0}\right)=0
$$

que é uma contradição pois supomos acima que $\operatorname{Lip}_{\infty}(f)>\inf _{s>0} R_{f}(s) \geq 0$.

O resultado a seguir nos dá condições para que uma função entre espaços normados envie pontos médio aproximados do domínio em pontos médio aproximados do codomínio.

Proposição 4.2.4. Sejam $X$ e $Y$ espaços normados e $f: X \rightarrow Y$ CLC tal que Lip $p_{\infty}(f)>0$. Então para todo $\delta>0, \epsilon>0$ e $d>0$ existem $x, y \in X$ com $\|x-y\|>d$ e tais que:

$$
f[\operatorname{Mid}(x, y, \delta)] \subset \operatorname{Mid}(f(x), f(y),(1+\epsilon) \delta) .
$$

Demonstração. Seja $0<\nu<1$ tal que

$$
\frac{1+\nu}{1-\nu}<\frac{1+(1+\epsilon) \delta}{1+\delta}
$$

Como $R_{f}$ é uma função decrescente, pelo Lema 4.2 .3 existe $s>d$ tal que $R_{f}(s)<(1+$ $\nu) \operatorname{Lip}_{\infty}(f)$. Sejam $x, y \in X$ tais que $\|x-y\| \geq 2 s(1-\delta)^{-1} \mathrm{e}$

$$
\|f(x)-f(y)\|>(1-\nu) R_{f}\left(2 s(1-\delta)^{-1}\right)\|x-y\| \geq(1-\nu) \operatorname{Lip}_{\infty}(f)\|x-y\| .
$$

Então para cada $u \in \operatorname{Mid}(x, y, \delta)$, temos que

$$
\min \{\|x-u\|,\|y-u\|\} \geq(1-\delta)\|x-y\| / 2 \geq(1-\delta) 2 s(1-\delta)^{-1} / 2=s,
$$

e assim

$$
\begin{aligned}
\max \{\|f(x)-f(u)\|,\|f(y)-f(u)\|\} & \leq R_{f}(s) \max \{\|x-u\|,\|y-u\|\} \\
& <(1+\nu) \operatorname{Lip}_{\infty}(f)(1+\delta)\|x-y\| / 2 .
\end{aligned}
$$

Por escolha, temos $\operatorname{Lip}_{\infty}(f)\|x-y\|<\|f(x)-f(y)\|(1-\nu)^{-1}$ e portanto

$$
\begin{aligned}
\max \{\|f(x)-f(u)\|,\|f(y)-f(u)\|\} & <(1+\nu)(1-\nu)^{-1}(1+\delta)\|f(x)-f(y)\| / 2 \\
& <(1+(1+\epsilon) \delta)\|f(x)-f(y)\| / 2 .
\end{aligned}
$$


Ou seja, $f(u) \in \operatorname{Mid}(f(x), f(y),(1+\epsilon) \delta)$.

\subsection{Princípio de Gorelik}

Enunciemos primeiramente o resultado abaixo.

Teorema 4.3.1 (Bourgain, Enflo, Gorelik). Se $1 \leq p<\infty$, então $\ell_{p}$ e $L_{p}$ não são uniformemente homeomorfos.

O caso $p=1$ foi provado por Enflo (não publicado) e o caso $1<p<2$ foi provado por Bourgain ([Bou83]). Gorelik provou o caso $2<p<\infty$ ([Gor94]) e para isso desenvolveu o que chamamos de princípio de Gorelik. O princípio nos diz, a grosso modo, que um homeomorfismo uniforme entre espaços de Banach não pode levar uma bola grande de um espaço de codimensão finita em uma vizinhança pequena de um espaço de codimensão infinita. Este princípio, junto ao que desenvolvemos sobre pontos médio aproximados, nos permitirá concluir que certos homeomorfismos uniformes entre espaços de Banach não podem existir. Para demonstrar o teorema, precisamos antes de dois lemas.

Lema 4.3.2. Sejam $Y$ um espaço de Banach, $Y_{0}$ um subespaço fechado de $Y$ de codimensão infinita e $K \subset Y$ um compacto. Então para todo $\tau>0$ existe $y \in Y$ tal que $\|y\|<\tau$ e $d\left(Y_{0}, K+y\right)>\tau / 2$.

Demonstração. Sejam $q: Y \rightarrow Y / Y_{0}$ a aplicação quociente e $x_{1}, \ldots, x_{n} \in K$ tais que $q[K] \subset$ $\bigcup_{i=1}^{n} B\left(\overline{x_{i}} ; \tau / 16\right)$. Como a dimensão de $Y / Y_{0}$ é infinita, o subespaço fechado $Z=\left\langle\left\{\overline{x_{1}}, \ldots, \overline{x_{n}}\right\}\right\rangle$ é próprio. Pelo Lema de Riez 2.3.3, existe $y \in Y$ tal que $\|\bar{y}\|=5 \tau / 6$ e $d(\bar{y}, Z) \geq 5 \tau / 8=\tau / 2+$ $\tau / 8$. Tome por fim $y_{0} \in \bar{y} \operatorname{com}\left\|y_{0}\right\|<\tau$. Para cada $x \in K$, tome $x_{i} \operatorname{com}\left\|\bar{x}-\overline{x_{i}}\right\|_{Y / Y_{0}}<\tau / 16$. Então

$$
\begin{aligned}
d\left(x+y_{0}, Y_{0}\right)=\left\|\bar{x}+\overline{y_{0}}\right\|_{Y / Y_{0}} & \geq\left\|\overline{x_{i}}+\bar{y}\right\|_{Y / Y_{0}}-\left\|\bar{x}-\overline{x_{i}}\right\|_{Y / Y_{0}} \\
& \geq \tau / 2+\tau / 8-\tau / 16 \\
& =\tau / 2+\tau / 16
\end{aligned}
$$

e portanto $d\left(K+y_{0}, Y_{0}\right)>\tau / 2$.

Lema 4.3.3. Seja $X$ um espaço de Banach e $X_{0}$ um subespaço fechado de codimensão finita. Então para todo $\tau>0$ existe um compacto $A \subset \tau B_{X}$ tal que para toda função $\varphi: A \rightarrow X$ continua satisfazendo $\|\varphi(x)-x\|<\tau / 2$ para cada $x \in A$, temos $\varphi[A] \cap X_{0} \neq \emptyset$.

Demonstração. Aplicando o Teorema 2.3.35 para $q: X \rightarrow X / X_{0}$ e $\lambda=\frac{4}{3}$, obtemos que existe $\phi^{\prime}: X / X_{0} \rightarrow X$ tal que $\phi^{\prime}(\bar{x}) \in \bar{x}$ para cada $\bar{x} \in X / X_{0}$ e $\left\|\phi^{\prime}(\bar{x})\right\| \leq \frac{4}{3}\|\bar{x}\|$ para cada $\bar{x} \in X$. Seja $\phi$ a restrição de $\phi^{\prime}$ ao conjunto $3 \tau / 4 B_{X / X_{0}}$. Obtemos então $\phi: 3 \tau / 4 B_{X / X_{0}} \rightarrow \tau B_{X}$ contínua tal que $\phi(\bar{x}) \in \bar{x}$ para cada $\bar{x} \in 3 \tau / 4 B_{X / X_{0}}$. Sejam $A=\phi\left[3 \tau / 4 B_{X / X_{0}}\right]$ e $\varphi: A \rightarrow X$ 
contínua satisfazendo $\|\varphi(x)-x\|<\tau / 2$ para cada $x \in A$. Defina $\psi: 3 \tau / 4 B_{X / X_{0}} \rightarrow X / X_{0}$ por $\psi(\bar{x})=\bar{x}-\overline{\varphi(\phi(\bar{x}))}$ para cada $\bar{x} \in 3 \tau / 4 B_{X / X_{0}}$ e veja que

$$
\|\psi(\bar{x})\|=\|\bar{x}-\overline{\varphi(\phi(\bar{x}))}\|=\|\overline{\phi(\bar{x})}-\overline{\varphi(\phi(\bar{x}))}\| \leq\|\phi(\bar{x})-\varphi(\phi(\bar{x}))\|<\tau / 2 .
$$

Portanto $\psi$ é uma função contínua com $\psi\left[3 \tau / 4 B_{X / X_{0}}\right] \subset 3 \tau / 4 B_{X / X_{0}}$, e pelo Teorema do ponto fixo de Schauder 2.3.36 existe $\bar{x} \in 3 \tau / 4 B_{X / X_{0}}$ tal que $\psi(\bar{x})=\bar{x}$. Assim, segue que $\overline{\varphi(\phi(\bar{x}))}=0$, isto é, $\varphi(\phi(\bar{x})) \in X_{0} \cap \varphi(A)$.

Teorema 4.3.4 (Princípio de Gorelik). Sejam X, Y espaços de Banach e $f: X \rightarrow Y$ um homeomorfismo entre $X$ e $Y$ tal que $f^{-1}$ é uniformemente contínuo. Suponha que existam $X_{0}$ subespaço fechado de codimensão finita de $X, Y_{1}$ subespaço fechado de codimensão infinita de $Y$ e b, $d>0$ tais que:

$$
f\left[d B_{X_{0}}\right] \subset Y_{1}+b B_{Y}
$$

Então $d / 4 \leq \omega_{f^{-1}}(2 b)$.

Demonstração. Suponha por contradição que $\omega_{f^{-1}}(2 b)<d / 4$ e seja $y \in Y$ com $\|y\|<2 b$. Definimos $\varphi: X \rightarrow X$ por $\varphi(x)=f^{-1}(f(x)+y)$. Note que

$$
\|\varphi(x)-x\|=\left\|f^{-1}(f(x)+y)-f^{-1}(f(x))\right\| \leq \omega_{f^{-1}}(\|y\|) \leq \omega_{f^{-1}}(2 b)<d / 4 .
$$

Aplicando 4.3.3 para $\tau=d / 2$, obtemos um compacto $A \subset \tau B_{X}$ e $a_{0} \in A$ tal que $\varphi\left(a_{0}\right) \in X_{0}$. Veja que para cada $a \in A$, temos

$$
\|\varphi(a)\| \leq\|a\|+d / 4 \leq d / 2+d / 4<d
$$

$\operatorname{logo} f^{-1}\left(f\left(a_{0}\right)+y\right)=\varphi\left(a_{0}\right) \in d B_{X_{0}}$. Segue que

$$
f\left(a_{0}\right)+y \in f\left[d B_{X_{0}}\right] \subset Y_{1}+b B_{Y},
$$

e então $d\left(f(A)+y, Y_{1}\right) \leq b$. Logo, aplicando o Lema 4.3.2 para $\tau=2 b$, obtemos uma contradição pois $y \in Y$ foi escolhido arbitrariamente em $B_{Y}(0 ; 2 b)$.

\subsection{Grafos Métricos}

Uma outra ferramenta que iremos usar é a de grafos métricos. Combinando com a teoria de Ramsey, conseguiremos estimativas que irão complementar a seção anterior. A principal referência da seção é [KR08].

Definição 4.4.1. Seja $\mathbb{M} \subset \mathbb{N}$ infinito. Definimos $G_{k}(\mathbb{M})$ como sendo o conjunto de todos subconjuntos de $\mathbb{M}$ com tamanho $k$. Além disso, equipamos $G_{k}(\mathbb{M})$ com a seguinte métrica: $d\left(\left(n_{1}, \ldots, n_{k}\right),\left(m_{1}, \ldots, m_{k}\right)\right)=\left|\left\{i: n_{i} \neq m_{i}\right\}\right|$ para todos $\left\{n_{1}, \ldots, n_{k}\right\},\left\{m_{1}, \ldots, m_{k}\right\} \in G_{k}(\mathbb{M})$, 
onde as $k$-tuplas acima estão ordenadas de maneira crescente.

Definição 4.4.2. Sejam $\mathbb{M} \subset \mathbb{N}$ infinito, $(M, d)$ um espaço métrico e $f: G_{k}(\mathbb{M}) \rightarrow M$ uma função. Dizemos que $\alpha \in M$ é o limite de $f$ se para cada $\epsilon>0$, existe $N \in \mathbb{N}$ tal que $A \in G_{k}(\mathbb{M})$ e $A \subset[N, \infty)$ implicam $d(f(A), \alpha)<\epsilon$.

\section{Observação 4.4.3.}

1. Caso exista o limite da função acima, este limite é único e o denotamos por $\lim _{A \in G_{k}(\mathbb{M})} f(A)$.

2. No caso em que $k=1$, existir o limite da função $f: \mathbb{M} \rightarrow M$ equivale a existir o limite da sequência $\left(f\left(m_{k}\right)\right)_{k \in \mathbb{N}}$, onde $\mathbb{M}=\left\{m_{k}: k \in \mathbb{N}\right.$ e $m_{k_{1}}<m_{k_{2}}$ para $k_{1}<$ $\left.k_{2} \operatorname{com} k_{1}, k_{2} \in \mathbb{N}\right\}$.

Teorema 4.4.4 (Ramsey). Sejam $k \in \mathbb{N},(K, d)$ um espaço métrico compacto e $f: G_{k}(\mathbb{N}) \rightarrow$ $K$ uma função. Então existe $\mathbb{M} \subset \mathbb{N}$ infinito para o qual existe o limite $\lim _{A \in G_{k}(\mathbb{M})} f(A)$.

Demonstração. Demonstraremos por indução em $k$. Para $k=1$, consideramos a sequência $(f(n))_{n \in \mathbb{N}}$, que é uma sequência em um compacto e então existe uma sequência crescente de naturais $\left(n_{k}\right)_{k \in \mathbb{N}}$ tal que $\left(f\left(n_{k}\right)\right)_{k \in \mathbb{N}}$ é convergente. O resultado segue da observação acima tomando $\mathbb{M}=\left\{n_{k}: k \in \mathbb{N}\right\}$. Suponha $k \geq 2$ e que vale o teorema para $k-1$. Podemos definir $\hat{f}\left(m_{1}, \ldots, m_{k}\right)=f\left(\left\{m_{1}, \ldots, m_{k}\right\}\right)$ sempre que $m_{1}, \ldots, m_{k}$ forem números naturais distintos entre si. Fixados $m_{1}, \ldots, m_{k-1}$ distintos, obtemos uma sequência $\left(\hat{f}\left(m_{1}, \ldots, m_{k}\right)\right)_{m_{k} \in \mathbb{N} /\left\{m_{1}, \ldots, m_{k-1}\right\}}$. Portanto existe $M_{1} \subset \mathbb{N}$ tal que existe

$$
g\left(\left\{m_{1}, \ldots, m_{k-1}\right\}\right)=\lim _{m_{k} \in M_{1} /\left\{m_{1}, \ldots, m_{k-1}\right\}} \hat{f}\left(m_{1}, \ldots, m_{k}\right)
$$

para cada $\left\{m_{1}, \ldots, m_{k-1}\right\} \in G_{k-1}(\mathbb{N})$. Pela hipótese de indução, existem $M_{2} \subset M_{1}$ infinito e $\alpha \in K$ tais que $\alpha=\lim _{A \in G_{k-1}\left(M_{2}\right)} g(A)$.

Se $A \in G_{k-1}\left(M_{2}\right)$ e $\epsilon>0$, pela definição de $g$ podemos encontrar $N=N(A, \epsilon) \in \mathbb{N}$ tal que $n \in M_{2}$ e $n \geq N$ implicam $n \notin A$ e $d(f(A \cup\{n\}), g(A))<\epsilon$. Sejam agora $m_{1}<\ldots<m_{k}$ em $M_{2}$. Para cada $n>k-1$, tomamos $m_{n+1}>m_{n}$ tal que

$$
m_{n+1}>\max \left\{N\left(A, 2^{-n}\right): A \in G_{k-1}\left\{m_{1}, \ldots, m_{n}\right\}\right\}
$$

Seja então $\mathbb{M}=\left\{m_{n}: n \in \mathbb{N}\right\}$ e provemos que $\alpha=\lim _{A \in G_{k}(\mathbb{M})} f(A)$. De fato, seja $\epsilon>0$ e $n \in \mathbb{N}$ tal que $A \in G_{k-1}(\mathbb{M})$ e $A \subset\left[m_{n}, \infty\right)$ implicam $d(g(A), \alpha)<\epsilon / 2$ e que seja grande o suficiente de modo que $2^{-n}<\epsilon / 2$. Seja agora $B \in G_{k}(\mathbb{M})$ com $B \subset\left[m_{n}, \infty\right)$. Tome $m_{r}=\max B$ e seja $A=B \backslash\left\{m_{r}\right\}$. Por construção, temos

$$
d(f(B), g(A))=d\left(f\left(A \cup\left\{m_{r}\right\}\right), g(A)\right)<2^{-(r-1)} \leq 2^{-n}<\epsilon / 2
$$

e portanto $d(f(A), \alpha)<\epsilon$. 
Lema 4.4.5. Sejam $X$ um espaço de Banach, $\mathbb{M} \subset \mathbb{N}$ infinito e $f: G_{k}(\mathbb{M}) \rightarrow X$ uma função. Suponha que existam $K \subset X$ compacto e $\delta>0$ tais que $f\left(G_{k}(\mathbb{M})\right) \subset K+\delta B_{X}$. Então para todo $\epsilon>0$, existe $\mathbb{M}^{\prime} \subset \mathbb{M}$ infinito tal que diamf $\left[G_{k}\left(\mathbb{M}^{\prime}\right)\right] \leq 2 \delta+\epsilon$.

Demonstração. Podemos escrever $f=g+h$, onde $g: G_{k}(\mathbb{M}) \rightarrow K$ e $h: G_{k}(\mathbb{M}) \rightarrow \delta B_{X}$. Aplicando o teorema acima para $g$ obtemos $\alpha \in X$ e $\mathbb{M}_{0} \subset \mathbb{M}$ infinito tais que $\alpha=\lim _{A \in G_{k}\left(\mathbb{M}_{0}\right)} g(A)$. Em particular, para cada $\epsilon>0$, existe $N \in \mathbb{N}$ tal que $A \in G_{k}\left(\mathbb{M}_{0}\right)$ e $A \subset[N, \infty)$ implicam $\|g(A)-\alpha\|<\epsilon / 2$. Seja então $\mathbb{M}^{\prime}=\mathbb{M}_{0} \cap\{n \in \mathbb{N}: n \geq N\}$. Então $\|g(A)-g(B)\|<\epsilon$ para todos $A, B \in G_{k}\left(\mathbb{M}^{\prime}\right)$ e segue que

$$
\sup _{A, B \in G_{k}\left(\mathbb{M}^{\prime}\right)}\|f(A)-f(B)\| \leq \sup _{A, B \in G_{k}\left(\mathbb{M}^{\prime}\right)}(\|h(A)-h(B)\|+\|g(A)-g(B)\|) \leq 2 \delta+\epsilon .
$$

Definição 4.4.6. Sejam $X$ um espaço de Banach reflexivo e $1<p<\infty$. Dizemos que $X$ tem a propriedade $P(p)$ se para todos $x \in X e\left(x_{n}\right)_{n \in \mathbb{N}}$ sequência fracamente convergente a 0 , temos:

$$
\limsup _{n \in \mathbb{N}}\left\|x+x_{n}\right\|^{p} \leq\|x\|^{p}+\limsup _{n \in \mathbb{N}}\left\|x_{n}\right\|^{p} .
$$

Teorema 4.4.7. Sejam $1<p<\infty$ e X um espaço de Banach reflexivo com a propriedade $P(p)$. Então se $\mathbb{M} \subset \mathbb{N}$ é infinito, $\epsilon>0$ e $f: G_{k}(\mathbb{M}) \rightarrow X$ é limitada, existe $\mathbb{M}^{\prime} \subset \mathbb{M}$ infinito tal que

$$
\operatorname{diamf}\left[G_{k}\left(\mathbb{M}^{\prime}\right)\right]<2 \operatorname{Lip}(f) k^{1 / p}+\epsilon .
$$

Demonstração. Vamos demonstrar por indução em $k$ que dados qualquer $f: G_{k}(\mathbb{M}) \rightarrow X$ limitada e $\epsilon>0$, existem $\mathbb{M}^{\prime} \subset \mathbb{M}$ infinito e $u \in X$ tais que:

$$
\left\|f\left(\left\{n_{1}, \ldots, n_{k}\right\}\right)-u\right\|<\operatorname{Lip}(f) k^{1 / p}+\epsilon / 2 \text { para cada }\left\{n_{1}, \ldots, n_{k}\right\} \in G_{k}\left(\mathbb{M}^{\prime}\right)
$$

Se $k=1, f$ é uma sequência e como $X$ é reflexivo, podemos encontrar $M_{0} \subset \mathbb{M}$ infinito para o qual existe o limite $u=\lim _{n \in M_{0}} f(\{n\})$ na topologia fraca. Logo, para cada $n \in M_{0}$, tome $x_{n}^{*} \in S_{X^{*}}$ tal que $x_{n}^{*}(f(\{n\})-u)=\|f(\{n\})-u\|\left(\right.$ tal $x_{n}^{*}$ existe por 2.3.7) e então

$$
\|f(\{n\})-u\|=\liminf _{m \in M_{0}}\left|x_{n}^{*}(f(\{n\})-f(\{m\}))\right| \leq \liminf _{m \in M_{0}}\|f(\{n\})-f(\{m\})\| \leq \operatorname{Lip}(f) .
$$

Suponha então que $k>1$ e que vale a sentença acima para $k-1$. Seja $f: G_{k}(\mathbb{M}) \rightarrow X$ limitada. Definindo $\hat{f}\left(n_{1}, \ldots, n_{k}\right)=f\left(\left\{n_{1}, \ldots, n_{k}\right\}\right)$ para cada $\left\{n_{1}, \ldots, n_{k}\right\} \in G_{k}(\mathbb{M})$, e fixados $n_{1}, \ldots, n_{k-1} \in \mathbb{M}$ distintos, obtemos uma sequência $\left(\hat{f}\left(n_{1}, \ldots, n_{k-1}, n_{k}\right)\right)_{n_{k} \in \mathbb{M} \backslash\left\{n_{1}, \ldots, n_{k-1}\right\}}$. Então, podemos encontrar $M_{0} \subset \mathbb{M}$ infinito tal que $g\left(\left\{n_{1}, \ldots, n_{k-1}\right\}\right)=\lim _{n_{k} \in M_{0}} \hat{f}\left(n_{1}, \ldots, n_{k}\right)$ existe fracamente para cada $\left\{n_{1}, \ldots, n_{k-1}\right\} \in G_{k-1}\left(M_{0}\right)$. Pela hipótese de indução, para cada $\delta>0$ existe $M_{1} \subset M_{0}$ infinito tal que para cada $\left\{n_{1}, \ldots, n_{k-1}\right\} \in G_{k-1}\left(M_{1}\right)$,

$$
\left\|g\left(\left\{n_{1}, \ldots, n_{k-1}\right\}\right)-u\right\|<\operatorname{Lip}(g)(k-1)^{1 / p}+\delta / 4 .
$$


Então para cada $\bar{n}=\left\{n_{1}, \ldots, n_{k-1}\right\} \in G_{k-1}\left(M_{1}\right)$ e pela propriedade $P(p)$ do espaço,

$$
\begin{aligned}
\limsup _{n_{k} \in M_{1}}\left\|f\left(\bar{n} \cup\left\{n_{k}\right\}\right)-u\right\|^{p} & =\limsup _{n_{k} \in M_{1}}\left\|f\left(\bar{n} \cup\left\{n_{k}\right\}\right)-g(\bar{n})+g(\bar{n})-u\right\|^{p} \\
& \leq\left(\operatorname{Lip}(g)(k-1)^{1 / p}+\delta / 4\right)^{p}+\limsup _{n_{k} \in M_{1}}\left\|f\left(\bar{n} \cup\left\{n_{k}\right\}\right)-g(\bar{n})\right\|^{p} .
\end{aligned}
$$

Mas $\limsup _{n_{k} \in M_{1}}\left\|f\left(\bar{n} \cup\left\{n_{k}\right\}\right)-g(\bar{n})\right\| \leq \limsup _{n_{k} \in M_{1}} \limsup _{m_{k} \in M_{1}}\left\|f\left(\bar{n} \cup\left\{n_{k}\right\}\right)-f\left(\bar{n} \cup\left\{m_{k}\right\}\right)\right\| \leq \operatorname{Lip}(f)^{p}$ e assim

$$
\limsup _{n_{k} \in M_{1}}\left\|f\left(\bar{n} \cup\left\{n_{k}\right\}\right)-u\right\| \leq\left(\left(\operatorname{Lip}(g)(k-1)^{1 / p}+\delta / 4\right)^{p}+\operatorname{Lip}(f)^{p}\right)^{1 / p} .
$$

Considere a função contínua $\phi: \mathbb{R} \rightarrow \mathbb{R}$ dada por

$$
\phi(t)=\left(\left(\operatorname{Lip}(g)(k-1)^{1 / p}+t / 4\right)^{p}+\operatorname{Lip}(f)^{p}\right)^{1 / p} .
$$

Afirmamos que $\operatorname{Lip}(g) \leq \operatorname{Lip}(f)$. De fato, sejam $\left\{n_{1}, \ldots, n_{k-1}\right\},\left\{m_{1}, \ldots, m_{k-1}\right\} \in G_{k-1}\left(M_{0}\right)$ e seja $x^{*} \in S_{X^{*}}$ tal que $x^{*}\left(g\left(\left\{n_{1}, \ldots, n_{k-1}\right\}\right)-g\left(\left\{m_{1}, \ldots, m_{k-1}\right\}\right)\right)=\| g\left(\left\{n_{1}, \ldots, n_{k-1}\right\}\right)-$ $g\left(\left\{m_{1}, \ldots, m_{k-1}\right\}\right) \|$. Então

$$
\begin{aligned}
\left\|g\left(\left\{n_{1}, \ldots, n_{k-1}\right\}\right)-g\left(\left\{m_{1}, \ldots, m_{k-1}\right\}\right)\right\| & =\left|x^{*}\left(g\left(\left\{n_{1}, \ldots, n_{k-1}\right\}\right)-g\left(\left\{m_{1}, \ldots, m_{k-1}\right\}\right)\right)\right| \\
& =\liminf _{n_{k} \in M_{0}}\left|x^{*}\left(f\left(\left\{n_{1}, \ldots, n_{k-1}, n_{k}\right\}\right)-f\left(\left\{m_{1}, \ldots, m_{k-1}, n_{k}\right\}\right)\right)\right| \\
& \leq \liminf _{n_{k} \in M_{0}}\left\|f\left(\left\{n_{1}, \ldots, n_{k-1}, n_{k}\right\}\right)-f\left(\left\{m_{1}, \ldots, m_{k-1}, n_{k}\right\}\right)\right\| \\
& \leq \operatorname{Lip}(f) d\left(\left(n_{1}, \ldots, n_{k-1}\right),\left(m_{1}, \ldots, m_{k-1}\right)\right) .
\end{aligned}
$$

Temos então que $\phi(0) \leq \operatorname{Lip}(f) k^{1 / p}$ e $\operatorname{logo} \phi(\delta)<\operatorname{Lip}(f) k^{1 / p}+\epsilon / 4$ para $\delta>0$ suficientemente pequeno. Segue então que

$$
\limsup _{n_{k} \in M_{1}}\left\|f\left(\bar{n} \cup\left\{n_{k}\right\}\right)-u\right\|<\operatorname{Lip}(f) k^{1 / p}+\epsilon / 4 .
$$

Defina $h: G_{k}\left(M_{1}\right) \rightarrow \mathbb{R}$ por $h(\bar{n})=\|f(\bar{n})-u\|$ para cada $\bar{n} \in G_{k}\left(M_{1}\right)$. Como $h$ é limitada, podemos aplicar o Teorema de Ramsey 4.4.4 para obter $\mathbb{M}^{\prime} \subset M_{1}$ infinito tal que $\mid\|f(\bar{n})-u\|-$ $\|f(\bar{m})-u\| \mid<\epsilon / 4$ para cada $\bar{n}, \bar{m} \in G_{k}\left(\mathbb{M}^{\prime}\right)$. Portanto $\left\|f\left(\left\{n_{1}, \ldots, n_{k}\right\}\right)-u\right\|<\operatorname{Lip}(f) k^{1 / p}+$ $\epsilon / 2$ para cada $\left\{n_{1}, \ldots, n_{k}\right\} \in G_{k}\left(\mathbb{M}^{\prime}\right)$ e isto conclui a indução e consequentemente a prova do teorema.

Definição 4.4.8. Sejam $1 \leq p<\infty$ e $x=\left(x_{n}\right)_{n \in \mathbb{N}} \in \ell_{p}$. Definimos o suporte da sequência $x$ por suppx $=\left\{n \in \mathbb{N}: x_{n} \neq 0\right\}$.

Lema 4.4.9. Sejam $1<p_{1}<p_{2}<\ldots<p_{m}$. Então o espaço $X=\left(\ell_{p_{1}} \oplus \ldots \oplus \ell_{p_{m}}\right)_{\infty}$ satisfaz a propriedade $P\left(p_{1}\right)$.

Demonstração. Vamos provar primeiro que $X=\ell_{q}$ satisfaz a propriedade $P(p)$ com $1<$ $p \leq q<\infty$. Sejam $x \in \ell_{q}$ e $\left(x_{n}\right)_{n \in \mathbb{N}}$ sequência fracamente convergente a 0 em $\ell_{q}$. Queremos 
provar que

$$
\limsup _{n \in \mathbb{N}}\left\|x+x_{n}\right\|_{\ell_{q}}^{p} \leq\|x\|_{\ell_{q}}^{p}+\limsup _{n \in \mathbb{N}}\left\|x_{n}\right\|_{\ell_{q}}^{p} .
$$

Seja $\left(z_{m}\right)_{m}=\left(x_{n_{m}}\right)_{m}$ uma subsequência de $\left(x_{n}\right)_{n \in \mathbb{N}}$ tal que $\lim _{m}\left\|x+z_{m}\right\|^{p}=\limsup _{n \in \mathbb{N}}\left\|x+x_{n}\right\|^{p}$ e $\lim _{m}\left\|z_{m}\right\|^{p}=\limsup _{n \in \mathbb{N}}\left\|x_{n}\right\|^{p}$. Vamos criar uma sequência $\left(y_{k}\right)_{k}$ em $\ell_{q}$ e uma subsequência $\left(z_{m_{k}}\right)_{k}$ de $\left(z_{m}\right)_{m}$ tais que $\left\|z_{m_{k}}-y_{k}\right\|_{\ell_{q}} \rightarrow 0$, onde cada $y_{k}$ tem suporte finito com respeito à base canônica e para cada $k \in \mathbb{N}$

$$
\max \left\{n \in \mathbb{N}: y_{k}(n) \neq 0\right\}<\min \left\{n \in \mathbb{N}: y_{k+1}(n) \neq 0\right\},
$$

isto é, os suportes são crescentes.

Seja $N_{1}$ tal que $\left\|z_{1}-\sum_{i=1}^{N_{1}} z_{1}(i) e_{i}\right\|_{\ell_{q}}<1 / 2$. Definimos $m_{1}=1$ e $y_{1}=\sum_{i=1}^{N_{1}} z_{1}(i) e_{i}$. Como $z_{m} \rightarrow 0$ fracamente quando $m \rightarrow \infty$, segue que $\lim _{m} z_{m}(i)=0$ para cada $i \in \mathbb{N}$ e assim conseguimos encontrar $m_{2}$ tal que $\left\|\sum_{i=1}^{N_{1}} z_{m_{2}}(i) e_{i}\right\|_{\ell_{q}}<1 / 2^{3}$. Encontramos $N_{2}>N_{1}$ tal que $\left\|z_{m_{2}}-\sum_{i=1}^{N_{2}} z_{m_{2}}(i) e_{i}\right\|_{\ell_{q}}<1 / 2^{3}$. Então

$$
\left\|z_{m_{2}}-\sum_{i=N_{1}+1}^{N_{2}} z_{m_{2}}(i) e_{i}\right\|_{\ell_{q}}<1 / 2^{2} .
$$

Fazemos $y_{2}=\sum_{i=N_{1}+1}^{N_{2}} z_{m_{2}}(i) e_{i}$. Construimos então indutivamente $\left(m_{k}\right)_{k}$ e $\left(N_{k}\right)_{k}$ tais que

$$
\left\|z_{m_{k}}-\sum_{i=N_{k-1}+1}^{N_{k}} z_{m_{k}}(i) e_{i}\right\|_{\ell_{q}}<1 / 2^{k}
$$

e fazemos $y_{k}=\sum_{i=N_{k-1}}^{N_{k}} z_{m_{k}}(i) e_{i}$.

Sejam $\epsilon>0$ e $k$ suficientemente grande tal que

$$
\left(\left\|x+y_{k}\right\|_{\ell_{q}}+\left\|z_{m_{k}}-y_{k}\right\|_{\ell_{q}}\right)^{p} \leq\left\|x+y_{k}\right\|_{\ell_{q}}^{p}+\epsilon .
$$

Então $\left\|x+z_{m_{k}}\right\|_{\ell_{q}}^{p} \leq\left\|x+y_{k}\right\|_{\ell_{q}}^{p}+\epsilon$. Por outro lado, seja $\overline{x_{k}}=\sum_{i \in \text { suppy }} x(i) e_{i}$. Note que $x_{k} \rightarrow 0$ quando $k \rightarrow \infty$. Temos então

$$
\begin{aligned}
\left\|x+y_{k}\right\|_{\ell_{q}}^{p} & =\left(\sum_{i \notin \text { suppy }}|x(i)|^{q}+\sum_{i \in \text { suppy }}\left|x(i)+y_{k}(i)\right|^{q}\right)^{p / q} \\
& =\left(\left\|x-\overline{x_{k}}\right\|_{\ell_{q}}^{q}+\left\|\overline{x_{k}}+y_{k}\right\|_{\ell_{q}}^{q}\right)^{p / q} \\
& \leq\left\|x-\overline{x_{k}}\right\|_{\ell_{q}}^{p}+\left\|\overline{x_{k}}+y_{k}\right\|_{\ell_{q}}^{p} .
\end{aligned}
$$


Mas esta última expressão tende a $\|x\|_{\ell_{q}}^{p}+\lim _{k}\left\|y_{k}\right\|_{\ell_{q}}^{p}=\|x\|_{\ell_{q}}^{p}+\limsup _{n \in \mathbb{N}}\left\|x_{n}\right\|_{\ell_{q}}^{p}$. Portanto

$$
\limsup _{n \in \mathbb{N}}\left\|x+x_{n}\right\|_{\ell_{q}}^{p}=\lim _{k}\left\|x+z_{m_{k}}\right\|_{\ell_{q}}^{p} \leq \lim _{k}\left\|x+y_{k}\right\|_{\ell_{q}}^{p}+\epsilon \leq\|x\|_{\ell_{q}}^{p}+\limsup _{n \in \mathbb{N}}\left\|x_{n}\right\|_{\ell_{q}}^{p}+\epsilon .
$$

Como $\epsilon$ é arbitrário, o resultado segue.

Considere agora o espaço $X=\left(\ell_{p_{1}} \oplus \ldots \oplus \ell_{p_{m}}\right)_{\infty}$, onde $1<p_{1}<p_{2}<\ldots<p_{m}$. Sejam $x \in X$ e $\left(x_{n}\right)_{n \in \mathbb{N}}$ umas sequência fracamente convergente a 0 em $X$. Vamos escrever $x=$ $\left(x^{(1)}, \ldots, x^{(m)}\right)$ e $x_{n}=\left(x_{n}^{(1)}, \ldots, x_{n}^{(m)}\right)$ para cada $n \in \mathbb{N}$. Então $\left(x_{n}^{(j)}\right)_{n \in \mathbb{N}}$ é uma sequência fracamente convergente a 0 em $\ell_{p_{j}}$ para cada $j=1, \ldots, m$. Pelo que provamos acima, temos

$$
\limsup _{n \in \mathbb{N}}\left\|x^{(j)}+x_{n}^{(j)}\right\|_{\ell_{p_{j}}}^{p_{1}} \leq\|x\|_{\ell_{p_{j}}}^{p_{1}}+\limsup _{n \in \mathbb{N}}\left\|x_{n}\right\|_{\ell_{p_{j}}}^{p_{1}} \text { para cada } j=1, . ., m
$$

Segue então que

$$
\begin{aligned}
\limsup _{n \in \mathbb{N}}\left\|x+x_{n}\right\|_{X}^{p_{1}} & =\max _{j=1, \ldots, m}\left(\limsup _{n \in \mathbb{N}}\left\|x^{(j)}+x_{n}^{(j)}\right\|_{\ell_{p_{j}}}^{p_{1}}\right) \\
& \leq \max _{j=1, \ldots, m}\left(\left\|x^{(j)}\right\|_{\ell_{p_{j}}}^{p_{1}}+\limsup _{n \in \mathbb{N}}\left\|x_{n}^{(j)}\right\|_{\ell_{p_{j}}}^{p_{1}}\right) \\
& \left.\leq \max _{j=1, \ldots, m}\left(\left\|x^{(j)}\right\|_{\ell_{p_{j}}}^{p_{1}}\right)+\max _{j=1, \ldots, m}\left(\limsup _{n \in \mathbb{N}}\left\|x_{n}^{(j)}\right\|_{\ell_{p_{j}}}^{p_{1}}\right)\right) \\
& =\|x\|_{X}+\limsup _{n \in \mathbb{N}}\left\|x_{n}\right\|_{X} .
\end{aligned}
$$

\subsection{Estrutura Uniforme dos espaços $\ell_{p}$}

Agora que desenvolvemos as ferramentas necessárias, vamos estudar algumas propriedades dos espaços $\ell_{p}$ 's que irão possibilitar a aplicação das seções anteriores.

Definição 4.5.1. Sejam $X$ um espaço de Banach, $1 \leq p<\infty$ e $\left(e_{n}\right)_{n \in \mathbb{N}}$ uma base incondicional de $X$. Dizemos que esta base tem estimativa superior $p$ se existir uma constante $0<C<\infty$ tal que para qualquer sequência finita $\left(x_{k}\right)_{k=1}^{m}$ de vetores disjuntos com respeito $\grave{a}$ base $\left(e_{n}\right)_{n \in \mathbb{N}}$, vale:

$$
\left\|\sum_{k=1}^{m} x_{k}\right\|^{p} \leq C^{p} \sum_{k=1}^{m}\left\|x_{k}\right\|^{p} .
$$

Analogamente, dizemos que a base tem estimativa inferior $p$ se existir uma constante $0<$ $C<\infty$ tal que para qualquer sequência finita $\left(x_{k}\right)_{k=1}^{m}$ de vetores disjuntos com respeito à base $\left(e_{n}\right)_{n \in \mathbb{N}}$, vale:

$$
\left\|\sum_{k=1}^{m} x_{k}\right\|^{p} \geq C^{p} \sum_{k=1}^{m}\left\|x_{k}\right\|^{p} .
$$

Exemplo 4.5.2. Sejam $1 \leq p<\infty, X=\ell_{p} e\left(e_{n}\right)_{n \in \mathbb{N}}$ a base canônica do $\ell_{p}$. Então toda 
sequência $\left(x_{k}\right)_{k=1}^{m}$ disjunta com respeito à base satisfaz $\left\|\sum_{k=1}^{m} x_{k}\right\|^{p}=\sum_{k=1}^{m}\left\|x_{k}\right\|^{p}$. Logo $\left(e_{n}\right)_{n \in \mathbb{N}}$ tem estimativas superior e inferior $p$ e com constante $C \stackrel{k=1}{=}$.

Definição 4.5.3. Sejam $X$ um espaço com base incondicional $\left(e_{n}\right)_{n \in \mathbb{N}}$ e $x, y \in X$.Dizemos que $y \perp x$ sempre que os suportes de y e $x$ forem disjuntos com respeito à base $\left(e_{n}\right)_{n \in \mathbb{N}}$. Se $X_{1}$ é um subespaço de $X$, definimos ainda $X_{1}^{\perp}=\left\{y \in X: y \perp x\right.$ para cada $\left.x \in X_{1}\right\}$.

Definição 4.5.4. Sejam $1 \leq r<\infty$ e $N \in \mathbb{N}$. Definimos o subespaço $E_{N}$ de $\ell_{r}$ por

$$
E_{N}=\left[e_{j}\right]_{j>N}=\left\{\left(x_{n}\right)_{n \in \mathbb{N}} \in \ell_{r}: x_{1}=\ldots=x_{N}=0\right\}
$$

Definição 4.5.5. Sejam $1 \leq p<\infty$ e $n \in \mathbb{N}$. Definimos a n-ésima projeção de $\ell_{p}$ como sendo a função $P_{n}: \ell_{p} \rightarrow\left[e_{1}, \ldots, e_{n}\right]$ dada por $P_{n}(x)=\sum_{i=1}^{n} x(i) e_{i}$. Definimos analogamente a $n$-ésima projeção de $c_{0}$.

O lema a seguir mostra algumas relações entre os pontos médio aproximados dos espaços $\ell_{p}$ 's e do espaço $c_{0}$ com subconjuntos clássicos de espaços normados.

Lema 4.5.6. Sejam $X$ espaço de Banach e $\left(e_{n}\right)_{n \in \mathbb{N}}$ uma base incondicional de $X$ com constante igual a 1. Temos:

1. Se $\left(e_{n}\right)_{n \in \mathbb{N}}$ tem estimativa superior $1 \leq p<\infty$ com constante $C=1$, então para todo $\delta>0$ e para cada $x \in X$ existe um subespaço $X_{0}$ de codimensão finita tal que:

$$
\delta^{1 / p}\|x\| B_{X_{0}} \subset \operatorname{Mid}(x,-x, \delta)
$$

2. Se $\left(e_{n}\right)_{n \in \mathbb{N}}$ tem estimativa inferior $1 \leq r<\infty$ com constante $C=1$, então para todo $\delta>0$ suficientemente pequeno e para cada $x \in X$ existe um subespaço $X_{1}$ de dimensão finita tal que:

$$
\operatorname{Mid}(x,-x, \delta) \subset X_{1}+(3 r \delta)^{1 / r}\|x\| B_{X}
$$

3. Se $X=c_{0}$, para cada $x \in X$ com $x \neq 0$ e $0<\epsilon<\delta$, existe um subespaço $X_{0}$ de $X$ de codimensão finita tal que:

$$
(1+\epsilon)\|x\| B_{X_{0}} \subset \operatorname{Mid}(x,-x, \delta)
$$

Demonstração. 1) Sejam $x \in X$ e $\delta>0$. Considere primeiro o caso em que $p>1$ e $x$ tem suporte finito com respeito à base $\left(e_{n}\right)_{n \in \mathbb{N}}$. Tome $X_{0}(x)=X_{0}=\{y \in X: y \perp x\}$. Então para cada $y \in \delta^{1 / p}\|x\| B_{X_{0}}$, temos

$$
\|y \pm x\| \leq\left(\|y\|^{p}+\|x\|^{p}\right)^{1 / p} \leq(1+\delta)^{1 / p}\|x\|<(1+\delta)\|x\|
$$


Seja $x \in X$ qualquer, $\epsilon=(1+\delta)\|x\|-(1+\delta)^{1 / p}\|x\|$ e $z=P_{n}(x)$, onde $n$ é suficientemente grande tal que $\left\|P_{n}(x)-x\right\|<\epsilon$. Como a constante da base é 1 , temos $\|z\| \leq\|x\|$. Logo para cada $y \in \delta^{1 / p}\|x\| B_{X_{0}(z)}$, temos

$$
\|y \pm x\| \leq\|y \pm z\|+\epsilon<\left(\delta\|x\|^{p}+\|z\|^{p}\right)^{1 / p}+\epsilon \leq(1+\delta)^{1 / p}\|x\|+\epsilon=(1+\delta)\|x\| .
$$

Portanto $\max \{\|y+x\|,\|y-x\|\} \leq(1+\delta)\|x\|$, isto é, $y \in \operatorname{Mid}(x,-y, \delta)$.

Se $p=1$, para qualquer $y \in \delta\|x\| B_{X}$ temos $\|y \pm x\| \leq(1+\delta)\|x\|$ e assim $\delta B_{X} \subset$ $\operatorname{Mid}(x,-x, \delta)$.

2) Seja $0<\delta \leq 2^{1 /(r-1)}-1$. Suponha primeiramente que $x \in X_{1}=\left\langle\left\{e_{1}, \ldots, e_{N}\right\}\right\rangle$ para algum $N \in \mathbb{N}$ e $\|x\|=1$. Seja $y \in X$ tal que $\|y \pm x\| \leq 1+\delta$. Escrevendo $y=y_{1}+y_{2}$ com $y_{1} \in X_{1}$ e $y_{2} \in X_{1}^{\perp}$, vamos mostrar que $\left\|y_{2}\right\| \leq(2 r \delta)^{1 / 2}$. Como

$$
2=\|2 x\|=\left\|x-y_{1}+x+y_{1}\right\| \leq\left\|x-y_{1}\right\|+\left\|x+y_{1}\right\|,
$$

temos $\max \left\{\left\|x-y_{1}\right\|,\left\|x+y_{1}\right\|\right\} \geq 1$ e assim existe um sinal $\theta \in\{-1,1\}$ tal que $\left\|x+\theta y_{1}\right\| \geq 1$. Portanto

$$
1+\left\|y_{2}\right\|^{r} \leq\left\|x+\theta y_{1}\right\|^{r}+\left\|y_{2}\right\|^{r} \leq\left\|\theta x+y_{1}+y_{2}\right\|^{r} \leq(1+\delta)^{r} .
$$

Como $\delta \leq 2^{1 /(r-1)}-1$, temos $(1+\delta)^{r}-1 \leq 2 r \delta$, logo $y_{2} \in(2 r \delta)^{1 / r} B_{X}$.

Suponhamos agora que $x \in X$ é qualquer com $\|x\|=1$. Seja $n$ suficientemente grande tal que $\left\|x-P_{n}(x)\right\|<\delta / 4$. Sejam $z=P_{n}(x)$ e $y \in \operatorname{Mid}(x,-x, \delta)$. Veja que

$$
1-\|z\| \leq\|x-z\|<\delta / 4
$$

Então

$$
\begin{aligned}
\|y \pm z /\| z\|\| \leq\|y \pm z\|+\|z-z /\| z\|\| \| & <\|y \pm x\|+\|x-z\|+\|z-z /\| z\|\| \\
& <1+\delta+\delta / 4+1-\|z\| \\
& \leq 1+3 \delta / 2 .
\end{aligned}
$$

Portanto $y \in \operatorname{Mid}(z /\|z\|,-z /\|z\|, 3 \delta / 2) \subset X_{1}+(3 r \delta)^{1 / r} B_{X}$.

3) Sejam $0<\epsilon<\delta$. Considere primeiro o caso em que $x \in S_{c_{0}}$ tem suporte finito com respeito à base canônica. Seja então $N \in \mathbb{N}$ tal que $x \in\left[e_{n}: n<N\right]$. Defina $X_{0}$ por $\left[e_{n}: n \geq N\right]$ e seja $y \in(1+\epsilon) B_{X_{0}}$. Então $\|x \pm y\| \leq 1+\epsilon$, e como $1+\epsilon=(1+\epsilon)\|x-(-x)\| / 2$, temos que $y \in \operatorname{Mid}(x,-x, \epsilon) \subset \operatorname{Mid}(x,-x, \delta)$.

Seja agora $x \in S_{X}$ qualquer e $N \in \mathbb{N}$ tal que $\left\|P_{N}(x)-x\right\|<(\delta-\epsilon) / 2$. Sejam $z=P_{N}(x)$ e $X_{0}=\left[e_{n}: n \geq N+1\right]$, ou seja, $X_{0}$ é o subespaço dado acima para $z$. Seja $y \in(1+\epsilon) B_{X_{0}}$. 
Então

$$
\begin{aligned}
\|y \pm x\| \leq\|y \pm z /\| z\|\|+\|z /\| z\|-x\| & \leq 1+\epsilon+\|x-z\|+1-\|z\| \\
& \leq 1+\epsilon+2\|x-z\| \\
& \leq 1+\epsilon+\delta-\epsilon \\
& \leq 1+\delta .
\end{aligned}
$$

Portanto $y \in \operatorname{Mid}(x,-x, \delta)$.

\section{Observação 4.5.7.}

1. Note que em 1) podemos substituir o subespaço $X_{0}$ por algum $E_{N}$ pois para $N$ suficientemente grande temos $E_{N} \subset X_{0}$.

2. Note que em 2) podemos substituir $X_{1}$ por um conjunto compacto $K$ pois a primeira coordenada $y_{1}$ na demonstração é limitada.

No caso em que $X$ é uma soma de espaços $\ell_{p}$ 's, temos o seguinte resultado.

Lema 4.5.8. Sejam $1 \leq p_{1}<p_{2}<\ldots<p_{n}<\infty$ e $X=\left(\sum_{i=1}^{n} \ell_{p_{i}}\right)_{\ell_{p_{n}}}$. Seja $x \in X$ e $0<\delta<1$.

Então existe um compacto $K \subset X$ tal que $\operatorname{Mid}(x,-x, \delta) \subset K+\left(3 p_{n} \delta\right)^{1 / p_{n}}\|x\| B_{X}$.

Demonstração. Vamos provar para o caso $n=2$ e $X=\left(\ell_{p} \oplus \ell_{q}\right)_{\ell_{q}} \operatorname{com} p<q$. Seja $\left(f_{n}\right)_{n \in \mathbb{N}}$ a base de $X$ dada por

$$
f(n)=\left\{\begin{array}{ll}
\left(e_{\frac{n-1}{2}+1}, 0\right) & \text { se } n \text { é impar } \\
\left(0, e_{n / 2}\right) & \text { se } n \text { é par }
\end{array} \text { para cada } n \in \mathbb{N} .\right.
$$

Sejam $x_{1}, \ldots, x_{l} \in X$ disjuntos com respeito à base $\left(f_{n}\right)$. Vamos provar que $\left\|x_{1}+\ldots+x_{l}\right\|^{q} \geq$ $\left\|x_{1}\right\|^{q}+\ldots+\left\|x_{l}\right\|^{q}$, e com isso mostramos que a base $\left(f_{n}\right)_{n \in \mathbb{N}}$ tem estimativa inferior $q$ com constante $C=1$. O resultado vai seguir então de 4.5.6 2).

Escrevendo $x_{i}=\left(x_{i}^{\prime}, x_{i}^{\prime \prime}\right)$, obtemos que

$$
\left\|x_{1}+\ldots+x_{l}\right\|^{q}=\left\|x_{1}^{\prime}+\ldots+x_{l}^{\prime}\right\|_{\ell_{p}}^{q}+\left\|x_{1}^{\prime \prime}+\ldots+x_{l}^{\prime \prime}\right\|_{\ell_{q}}^{q}
$$

Mas como $x_{1}, \ldots, x_{l} \in X$ são disjuntos com respeito à base $\left(f_{n}\right)$, temos

$$
\begin{aligned}
\left\|x_{1}^{\prime}+\ldots+x_{l}^{\prime}\right\|_{\ell_{p}}^{q} & =\left(\sum_{n=1}^{\infty}\left|x_{1}^{\prime}(n)+\ldots+x_{l}^{\prime}(n)\right|^{p}\right)^{q / p} \\
& =\left(\sum_{n=1}^{\infty}\left|x_{1}^{\prime}(n)\right|^{p}+\ldots+\sum_{n=1}^{n}\left|x_{l}^{\prime}(n)\right|^{p}\right)^{q / p} \\
& =\left(\left\|x_{1}^{\prime}\right\|_{\ell_{p}}^{p}+\ldots+\left\|x_{l}^{\prime}\right\|_{\ell_{p}}^{p}\right)^{q / p} \\
& \geq\left\|x_{1}^{\prime}\right\|_{\ell_{p}}^{q}+\ldots+\left\|x_{l}^{\prime}\right\|_{\ell_{p}}^{q}
\end{aligned}
$$


pois $p<q$. De forma análoga, provamos que $\left\|x_{1}^{\prime \prime}+\ldots+x_{l}^{\prime \prime}\right\|_{\ell_{q}}^{q}=\left\|x_{1}^{\prime \prime}\right\|_{\ell_{q}}^{q}+\ldots+\left\|x_{l}^{\prime \prime}\right\|_{\ell_{q}}^{q}$. Portanto, segue que $X$ tem estimativa inferior $q$ com constante $C=1$.

Unindo o resultado anterior à Proposição 4.2.4, obtemos a seguinte proposição.

Proposição 4.5.9. Sejam $1 \leq p_{1}, p_{2}, \ldots, p_{n}<r<\infty, X=\left(\sum_{i=1}^{n} \ell_{p_{i}}\right)_{\ell_{p_{n}}}$ e $f: \ell_{r} \rightarrow X$ uma função CLC. Então para cada $t, \delta>0$ existem $x \in \ell_{r}, \tau>t, N \in \mathbb{N}$ e $K \subset X$ compacto tais que

$$
f\left[x+\tau B_{E_{N}}\right] \subset K+\delta \tau B_{X}
$$

Demonstração. Suponha primeiramente que $\operatorname{Lip}_{\infty}(f)=0$. Então para cada $\delta, t>0$ existe $\tau>t$ tal que $\omega_{f}(\tau) / \tau<\delta$. Tomando $x=0$ e $K=\{f(0)\}$, obtemos que $\|f(y)-f(0)\|<\delta \tau$ para todo $y$ com $\|y\|<\tau$, isto é,

$$
f\left[\tau B_{\ell_{r}}\right] \subset K+\delta \tau B_{X}
$$

Podemos supor então que $\sup \omega_{f}(t) / t=C>0$ para algum $s>0$. Seja $\nu<1 / 2$ tal que $\left(6 p_{n}\right)^{1 / p_{n}} C \nu^{1 / p_{n}-1 / r}<\delta$. Pelo Lema 4.2.4 existem $x, y \in \ell_{r}$ tais que $\|x-y\|>\max \left\{s, 2 t \nu^{-1 / r}\right\}$ $\mathrm{e}$

$$
f[\operatorname{Mid}(x, y, \nu)] \subset \operatorname{Mid}(f(x), f(y), 2 \nu) .
$$

Vamos supor primeiramente que $y=-x$ e que $f(-x)=-f(x)$. Seja então $\tau=\nu^{1 / r}\|x\|$. Pela Proposição 4.5.6 1), existe $N \in \mathbb{N}$ tal que $\tau B_{E_{N}} \subset \operatorname{Mid}(x,-x, \nu)$ e pelo Lema 4.5.8 existe $K \subset X$ compacto tal que

$$
\operatorname{Mid}(f(x),-f(x), 2 \nu) \subset K+\left(6 p_{n} \nu\right)^{1 / p_{n}}\|f(x)\| B_{X}
$$

e assim

$$
f\left[\tau B_{E_{N}}\right] \subset K+\left(6 p_{n} \nu\right)^{1 / p_{n}}\|f(x)\| B_{X} .
$$

Mas como $\|x-(-x)\|>s$, temos $\|f(x)\| \leq C\|x\|=C \tau \nu^{-1 / r}$ e portanto

$$
\left(6 p_{n} \nu\right)^{1 / p_{n}}\|f(x)\| \leq\left(6 p_{n}\right)^{1 / p_{n}} C \nu^{1 / p_{n}-1 / r} \tau<\delta \tau,
$$

isto é, $f\left[\tau B_{E_{N}}\right] \subset K+\delta \tau B_{X}$.

Em geral, aplicamos a proposição para a função $\phi(w)=f(w+(y+x) / 2)-(f(x)+f(y)) / 2$ e obtemos que

$$
f\left[(y+x) / 2+\tau B_{E_{N}}\right] \subset(K-(f(x)+f(y)) / 2)+\delta \tau B_{X} .
$$

Vamos mostrar no exemplo a seguir como podemos aplicar tudo que desenvolvemos até aqui para provar que $l_{1}$ e $c_{0}$ não são uniformemente homeomorfos. 
Exemplo 4.5.10. $\ell_{1}$ e $c_{0}$ não são uniformemente homeomorfos.

Demonstração. Suponha por contradição que exista um homeomorfismo uniforme $f: c_{0} \rightarrow$ $\ell_{1}$. Então para cada $y, w \in \ell_{1}$, temos para $M=K_{f^{-1}}(2)$ :

$$
\left\|f^{-1}(y)-f^{-1}(w)\right\| \leq M \max \{\|x-y\|, 2\}
$$

Sejam $K=K_{f}(2), 0<\delta<\min \{1 / 2,1 /(120 K M)\}$ e $d \geq 2 /(15 K \delta)$. Por 4.2.4, existem $x, y \in c_{0}$ tais que $\|x-y\| \geq \max \{d, 2\} \mathrm{e}$

$$
f[\operatorname{Mid}(x, y, \delta)] \subset \operatorname{Mid}(f(x), f(y), 5 \delta) .
$$

Podemos então supor sem perda de generalidade que $y=-x$ e que $f(-x)=-f(x)$. De fato, basta considerar $\phi: c_{0} \rightarrow \ell_{1}$ dado por $\phi(w)=f(w+(y+x) / 2)-(f(x)+f(y)) / 2$, e então $\phi((y-x) / 2)=-\phi((x-y) / 2)$ e $\operatorname{Lip}_{\infty}(\phi)=\operatorname{Lip}_{\infty}(f)$.

Pelo Lema 4.5.6, existe um subespaço $X_{0}$ de $c_{0}$ de codimensão finita e existe um subespaço $Y_{1}$ de $\ell_{1}$ de codimensão infinita tais que $(1+\delta / 2)\|x\| B_{X_{0}} \subset \operatorname{Mid}(x,-x, \delta) \mathrm{e}$ $\operatorname{Mid}(f(x),-f(x), 5 \delta) \subset Y_{1}+15 \delta\|f(x)\| B_{\ell_{1}}$. Então

$$
f\left[(1+\delta / 2)\|x\| B_{X_{0}}\right] \subset f[\operatorname{Mid}(x,-x, \delta)] \subset \operatorname{Mid}(f(x),-f(x), 5 \delta) \subset Y_{1}+15 \delta\|f(x)\| B_{\ell_{1}}
$$

Assim, o princípio de Gorelik 4.3.4 pode ser aplicado e obtemos $(1+\delta / 2)\|x\| / 4 \leq \omega_{f^{-1}}(30\|f(x)\| \delta)$. Por escolha, $\|x-(-x)\| \geq 2$, assim $\|f(x)\| \leq K\|x\|$ e

$$
(1+\delta / 2)\|x\| / 4 \leq \omega_{f^{-1}}(30\|f(x)\| \delta) \leq \omega_{f^{-1}}(30 K\|x\| \delta)
$$

Veja que sempre que $t \geq 2$, temos $\omega_{f^{-1}}(t) \leq M t$, mas pela nossa escolha de $d$,

$$
30 K\|x\| \delta \geq 15 K d \delta \geq 2
$$

e assim

$$
\|x\| / 4 \leq(1+\delta / 2)\|x\| / 4 \leq \omega_{f^{-1}}(30 K\|x\| \delta) \leq 30 K M\|x\| \delta .
$$

Portanto $\delta \geq 1 / 120 K M$, uma contradição.

Teorema 4.5.11. Sejam $1<p<\infty$ e X um espaço de Banach com uma base incondicional com estimativa superior $p$. Seja $Y$ um espaço de Banach uniformemente homeomorfo a $X$. Então nenhum quociente de $Y$ pode ter uma base com estimativa inferior $r$ com $r<p$.

Demonstração. Sejam $U: X \rightarrow Y$ um homeomorfismo uniforme entre $X$ e $Y$ e $\left(x_{n}\right)_{n \in \mathbb{N}}$ uma base incondicional de $X$ com estimativa superior $p$. Suponha por contradição que exista um operador quociente $Q: Y \rightarrow Z$ (veja 2.3.14) tal que $Z$ tem uma base incondicional $\left(z_{n}\right)_{n \in \mathbb{N}}$ com estimativa inferior $r$ para algum $0<r<p$. Defina $S: X \rightarrow Z$ por $S=Q \circ U$. Depois de renormar os espaços podemos assumir sem perda de generalidade que as constantes das 
bases sejam 1 e que $\operatorname{Lip}_{\infty}(S)=1$. Dados $d>1$ e $\delta>0$, segue da Proposição 4.2 .4 que existem $x, y \in X \operatorname{com}\|x-y\|>d \mathrm{e}$

$$
S[\operatorname{Mid}(x, y, \delta)] \subset \operatorname{Mid}(S(x), S(y), 5 \delta) .
$$

Assim como no exemplo acima, podemos supor que $y=-x$ e $S(y)=-S(x)$. Segue do Lema 4.5.6 que:

$$
S\left[\delta^{1 / p}\|x\| B_{X_{0}}\right] \subset Z_{1}+(15 r \delta)^{1 / r}\|S(x)\| B_{Z},
$$

onde $X_{0}$ é um subespaço de $X$ de codimensão finita e $Z_{1}$ um subespaço de $Z$ de dimensão finita. Seja $Y_{1}=Q^{-1}\left[Z_{1}\right]$. Afirmamos que $Y_{1}$ tem codimensão infinita e que

$$
Q^{-1}\left[Z_{1}+(15 r \delta)^{1 / r}\|S(x)\| B_{Z}\right] \subset Y_{1}+(15 r \delta)^{1 / r}\|S(x)\| C_{Q} B_{Y}
$$

onde $C_{Q}$ é uma constante dependendo apenas de $Q$. De fato, suponha $\alpha \in \mathbb{R}$ e que $y \in$ $Q^{-1}\left[Z_{1}+\alpha B_{Z}\right]$. Entao $q(y)=z_{1}+\alpha z$, onde $z_{1} \in Z_{1}$ e $z \in B_{Z}$, e $\log \mathrm{o}$

$$
\inf _{y^{\prime} \in Q^{-1}[\{z\}]}\left\|y^{\prime}\right\| \leq\left\|T^{-1}\right\|\|z\| \leq\left\|T^{-1}\right\|
$$

onde $T: Y / \operatorname{ker} Q \rightarrow Z$ é o isomorfismo induzido por $Q$. Fazendo $C_{Q}=\left\|T^{-1}\right\|+1$, existe $y^{\prime} \in Q^{-1}[\{z\}]$ tal que $\left\|y^{\prime}\right\|<C_{Q}$ e assim $q\left(y-\alpha y^{\prime}\right) \in Z_{1}$, que por sua vez implica que $y \in Y_{1}+\alpha C_{Q} B_{Y}$. Temos então:

$$
\begin{aligned}
U\left[\delta^{1 / p}\|x\| B_{X_{0}}\right] \subset Q^{-1}\left[S\left[\delta^{1 / p}\|x\| B_{X_{0}}\right]\right] & \subset Q^{-1}\left[Z_{1}+(15 r \delta)^{1 / r}\|S(x)\| B_{Z}\right] \\
& \subset Y_{1}+(15 r \delta)^{1 / r}\|S(x)\| C_{Q} B_{Y}
\end{aligned}
$$

Podemos então aplicar o princípio de Gorelik para obtermos

$$
\delta^{1 / p}\|x\| / 4 \leq \omega_{U^{-1}}\left(2(15 r \delta)^{1 / r}\|S(x)\| C_{Q}\right) .
$$

Veja que $\|S(x)\| \leq K_{S}(1)$ e se $t>1, \omega_{U^{-1}}(t) \leq K_{U^{-1}}(1) t$, logo para $d$ suficientemente grande obtemos

$$
\delta^{1 / p}\|x\| / 4 \leq 2(15 r \delta)^{1 / r}\|x\| C_{Q} K_{S}(1) K_{U^{-1}}(1)
$$

uma contradição para $\delta$ suficientemente pequeno.

Também temos em mãos as ferramentas para demonstrar os dois seguintes teoremas.

Definição 4.5.12. Sejam $X$ e $Y$ espaços de Banach. Dizemos que $f: X \rightarrow Y$ é um mergulho $C L C$ se $f$ é uma função $C L C$ e para cada $t>0$, existe $C_{f}(t)>0$ tal que

$$
\|f(x)-f(y)\| \geq C_{f}(t)\|x-y\| \text { para cada } x, y \in X \operatorname{com}\|x-y\| \geq t .
$$

Teorema 4.5.13. Sejam $1<p<q<\infty$. Então não existe mergulho $C L C f: \ell_{q} \rightarrow \ell_{p}$. 
Demonstração. Suponha por contradição que exista tal função. Podemos supor sem perda de generalidade que existe $0<C \leq 1$ tal que $\|f(x)-f(y)\| \geq C\|x-y\|$ para todos $\|x-y\| \geq 1$. Seja $\delta<C / 3$. Pela Proposição 4.5.9 temos que existem $u \in \ell_{q}, \tau>1, N \in \mathbb{N}$ e $K \subset \ell_{p}$ compacto tais que $f\left(u+\tau B_{E_{N}}\right) \subset K+\delta \tau B_{\ell_{p}}$. Pelo Lema de Riesz 2.3.3 existe uma sequência $\left(u_{n}\right)_{n \in \mathbb{N}}$ em $u+\tau B_{E_{N}}$ tal que $\left\|u_{n}-u_{m}\right\| \geq 1$ para cada $n \neq m$. Cada $f\left(u_{n}\right)$ pode ser escrita como $k_{n}+\delta \tau v_{n}$, onde $k_{n} \in K$ e $v_{n} \in B_{\ell_{p}}$. Tomando se necessário uma subsequência convergente de $\left(k_{n}\right)_{n \in \mathbb{N}}$, podemos supor sem perda de generalidade que $\left\|k_{n}-k_{m}\right\| \leq \delta \tau$ para cada $n, m \in \mathbb{N}$. Temos assim

$$
\left\|f\left(u_{n}\right)-f\left(u_{m}\right)\right\| \leq\left\|k_{n}-k_{m}\right\|+\delta \tau\left\|v_{n}-v_{m}\right\| \leq 3 \delta \tau \text { para todos } n, m \in \mathbb{N} .
$$

Mas então $C \tau \leq\left\|f\left(u_{n}\right)-f\left(u_{m}\right)\right\| \leq 3 \delta \tau$ para $n \neq m$, contradizendo a escolha de $\delta$.

Teorema 4.5.14. Sejam $1<p<q<\infty$. Então não existe mergulho $C L C g: \ell_{p} \rightarrow \ell_{q}$.

Demonstração. Suponha por contradição que exista tal $g$. Podemos supor supor sem perda de generalidade que existe $C \geq 1$ tal que para todos $x, y \in \ell_{p}$ com $\|x-y\| \geq 1$

$$
\|x-y\| \leq\|g(x)-g(y)\| \leq C\|x-y\|
$$

Sejam agora $k \in \mathbb{N}$ e $\varphi: G_{k}(\mathbb{N}) \rightarrow \ell_{p}$ definida por $\varphi(\bar{n})=e_{n_{1}}+\ldots+e_{n_{k}}$, onde $\bar{n}=$ $\left(n_{1}, \ldots, n_{k}\right) \in G_{k}(\mathbb{N})$. Temos que

$$
1 \leq\|\varphi(\bar{n})-\varphi(\bar{m})\| \leq 2^{1 / p} d(\bar{n}, \bar{m})^{1 / p} \leq 2 d(\bar{n}, \bar{m})
$$

Então

$$
\|g(\varphi(\bar{n}))-g(\varphi(\bar{m}))\| \leq C\|\varphi(\bar{n})-\varphi(\bar{m})\| \leq 2 C d(\bar{n}, \bar{m})
$$

e assim $\operatorname{Lip}(g \circ \varphi) \leq 2 C$. Pelo Teorema 4.4.7 existe um subconjunto infinito $\mathbb{M}$ de $\mathbb{N}$ tal que $\operatorname{diam}(g \circ \varphi)\left[G_{k}(\mathbb{M})\right] \leq 6 C k^{1 / q}$. Mas $\operatorname{diam}(g \circ \varphi)\left[G_{k}(\mathbb{M})\right]=(2 k)^{1 / p}, \log (2 k)^{1 / p} \leq 6 C k^{1 / q} \mathrm{e}$

$$
\frac{1}{6 C} \leq \frac{2^{1 / p}}{6 C} \leq k^{1 / q-1 / p}
$$

que é uma contradição para $k$ suficientemente grande pois $1 / q-1 / p<0$.

Como consequência, temos o seguinte teorema de Bourgain que foi demonstrado primeiramente em ([Bou83]).

Teorema 4.5.15 (Bourgain). Seja $1<p<2$. Então não existe homeomorfismo uniforme $f: \ell_{2} \rightarrow \ell_{p}$ para o qual $f$ e $f^{-1}: f\left[\ell_{2}\right] \rightarrow \ell_{p}$ são $C L C$.

Vamos enunciar agora o teorema de Maurey, que é um teorema clássico na teoria de extensão de operadores. 
Teorema 4.5.16 (Maurey). Sejam X um espaço com tipo 2, $Y$ um espaço com cotipo 2 e $X_{0}$ um subespaço de $X$. Então para todo operador $T: X_{0} \rightarrow Y$, existe uma extensão $S: X \rightarrow Y$.

Demonstração. Ver [Mau74].

Corolário 4.5.17. Sejam $X$ um espaço com tipo 2, $Y$ um espaço com cotipo 2 e $X_{0}$ um subespaço de $X$. Suponha que $Y$ seja isomorfo a $X_{0}$. Então $X_{0}$ é complementado em $X$.

Demonstração. Seja $T: X_{0} \rightarrow Y$ um isomorfismo entre $X_{0}$ e $Y$. Pelo teorema de Maurey, existe uma extensão $S: X \rightarrow Y$ de $T$. Considere $P=T^{-1} \circ S: X \rightarrow X_{0}$. Então $P$ é contínua e para cada $x_{0} \in X_{0}$, temos

$$
P\left(x_{0}\right)=T^{-1}\left(S\left(x_{0}\right)\right)=T^{-1}\left(T\left(x_{0}\right)\right)=x_{0} .
$$

Logo $X_{0}$ é um subespaço complementado de $X$.

O resultado a seguir foi provado originalmente em [Rib78], mas vamos fazer uma demonstração utilizando técnicas de ultraprodutos como é feito em [HM82].

Teorema 4.5.18 (Ribe). Sejam $1<p<\infty$ e X um espaço de Banach uniformemente homeomorfo a um espaço $\mathcal{L}_{p}$. Então $X$ é um espaço $\mathcal{L}_{p}$.

Demonstração. Sejam $Y$ um espaço $\mathcal{L}_{p}$ ao qual $X$ é uniformemente homeomorfo e $\mathcal{U}$ um ultrafiltro não principal em $\mathbb{N}$. Então, pelo Teorema 4.1.18, $X_{\mathcal{U}}$ é Lipschitz isomorfo a $Y_{\mathcal{U}}$. Por 2.3.78, $Y_{\mathcal{U}}$ também é um espaço $\mathcal{L}_{p}$ e por 2.3.77 $Y_{\mathcal{U}}$ não é isomorfo ao espaço $\ell_{2}$ e existe um subespaço complementado $Z$ de $L_{p}$ tal que $Y_{\mathcal{U}}$ é isomorfo a $Z$. Então $X_{\mathcal{U}}$ é Lipschitz isomorfo a $Z$, que é um espaço reflexivo e assim $X_{\mathcal{U}}$ também é reflexivo. Pelo Teorema 3.5.13, $X_{\mathcal{U}}$ é isomorfo a um subespaço complementado de $Z$, e logo $X_{\mathcal{U}}$ é isomorfo a um subespaço complementado de $L_{p}$. Como $X_{\mathcal{U}}$ é reflexivo, assim será $X$ e logo $X$ é isomorfo a um subespaço complementado de $X_{\mathcal{U}}$ por 2.3.62, e portanto a um subespaço de $L_{p}$. Por fim, se $X$ é isomorfo a $\ell_{2}, Y$ seria isomorfo a $\ell_{2}$ por 4.1.19. Portanto $X$ é um espaço $\mathcal{L}_{p}$ por 2.3.77.

Teorema 4.5.19 (Johnson). Sejam $1<p<\infty$ e $X$ um espaço $\mathcal{L}_{p}$ separável tal que nenhum subespaço é isomorfo ao $\ell_{2}$. Então $X$ é isomorfo ao espaço $\ell_{p}$.

Demonstração. Ver [JO74].

Podemos finalmente demonstrar o principal teorema do capítulo.

Teorema 4.5.20. Seja $1<p<\infty$. Então todo espaço de Banach $X$ uniformemente homeomorfo a $\ell_{p}$ é linearmente isomorfo $a \ell_{p}$. 
Demonstração. Seja $X$ um espaço de Banach uniformemente homeomorfo a $\ell_{p}, \operatorname{com} p \neq 2$. Vamos supor por contradição que $\ell_{2}$ seja isomorfo a um subespaço de $X$. Suponhamos primeiramente $p>2$. Como o tipo de $X$ é o mesmo que o de $\ell_{p}$ por 4.1.21, que por sua vez tem tipo 2, segue do Corolário 4.5.17 que $\ell_{2}$ é isomorfo a um subespaço complementado de $X$. Mas então, $\ell_{2}$ é isomorfo a um quociente de $X$ e assim $X$ possui um quociente com base com estimativa inferior $2<p$, contradizendo o Teorema 4.5.11. Suponha agora $1<p<2$ e sejam $f: X \rightarrow \ell_{p}$ um homeomorfismo uniforme e $T: \ell_{2} \rightarrow X$ um isomorfismo na imagem. Então $f \circ T: \ell_{2} \rightarrow \ell_{p}$ é um homeomorfismo uniforme na imagem cuja inversa é CLC (pois é uma composição de funções CLC), contradizendo o Teorema de Bourgain 4.5.15. Então $X$ não possui cópia de $\ell_{2}$.

Segue do Teorema de Ribe 4.5.18 que $X$ é um espaço $\mathcal{L}_{p}$. Por fim, novamento pelo fato de $X$ não possuir cópias de $\ell_{2}$, segue do Teorema de Johnson 4.5.19 que $X$ é isomorfo ao $\ell_{p}$. No caso em que $p=2$, o resultado segue diretamente de 4.1.19.

\subsection{Estrutura Uniforme dos espaços $\ell_{p} \oplus \ell_{q}$}

Já desenvolvemos quase todas ferramentas na seção anterior para estudar a estrutura uniforme dos espaços $\ell_{p} \oplus \ell_{q}$. Vamos separar nos casos em que $1<p<q<2$ ou $2<p<$ $q<\infty$, estudados em [JLS96], e no caso em que $1<p<2<q<\infty$, estudados em [KR08]. Enunciemos antes os seguintes teoremas.

Lema 4.6.1. Sejam $X$ e $Y$ espaços de Banach e $\mathcal{U}$ um ultrafiltro não principal em $\mathbb{N}$. Então o espaço $(X \oplus Y)_{\mathcal{U}}$ é isométrico ao espaço $X_{\mathcal{U}} \oplus Y_{\mathcal{U}}$.

Demonstração. Vamos equipar as somas externas com a norma do máximo $\|\cdot\|_{\infty}$. Considere o operador $T: \ell_{\infty}(X \oplus Y) \rightarrow \ell_{\infty}(X) \oplus \ell_{\infty}(Y)$ dado por

$$
T\left(\left(x_{n}, y_{n}\right)_{n \in \mathbb{N}}\right)=\left(\left(x_{n}\right)_{n \in \mathbb{N}},\left(y_{n}\right)_{n \in \mathbb{N}}\right) \text { para cada }\left(x_{n}, y_{n}\right)_{n \in \mathbb{N}} \in \ell_{\infty}(X \oplus Y) .
$$

Então $T$ é uma isometria pois

$$
\begin{aligned}
\left\|T\left(\left(x_{n}, y_{n}\right)_{n \in \mathbb{N}}\right)\right\|_{\infty} & =\left\|\left(\left(x_{n}\right)_{n \in \mathbb{N}},\left(y_{n}\right)_{n \in \mathbb{N}}\right)\right\|_{\infty} \\
& =\max \left\{\sup _{n \in \mathbb{N}}\left\|x_{n}\right\|, \sup _{n \in \mathbb{N}}\left\|y_{n}\right\|\right\} \\
& =\sup _{n \in \mathbb{N}}\left(\max \left\{\left\|x_{n}\right\|,\left\|y_{n}\right\|\right\}\right) \\
& =\sup _{n \in \mathbb{N}}\left\|\left(x_{n}, y_{n}\right)\right\|_{X \oplus Y} \\
& =\left\|\left(x_{n}, y_{n}\right)_{n \in \mathbb{N}}\right\|_{\ell_{\infty}(X \oplus Y) .}
\end{aligned}
$$

Além disso, $T$ é sobrejetora. Para terminar a demonstração, vamos mostrar que $T\left[c_{0, \mathcal{U}}(X \oplus\right.$ 
$Y)]=c_{0, \mathcal{U}}(X) \oplus c_{0, \mathcal{U}}(Y)$. De fato, para cada $\epsilon>0$, temos que

$$
\begin{aligned}
\left\{n \in \mathbb{N}:\left\|\left(x_{n}, y_{n}\right)\right\|_{X \oplus Y}<\epsilon\right\} & =\left\{n \in \mathbb{N}: \max \left\{\left\|x_{n}\right\|,\left\|y_{n}\right\|\right\}<\epsilon\right\} \\
& =\left\{n \in \mathbb{N}:\left\|x_{n}\right\|<\epsilon\right\} \cap\left\{n \in \mathbb{N}:\left\|y_{n}\right\|<\epsilon\right\}
\end{aligned}
$$

$\operatorname{assim}\left(x_{n}, y_{n}\right)_{n \in \mathbb{N}} \in c_{0, \mathcal{U}}(X \oplus Y)$ se e somente se $\left(x_{n}\right)_{n \in \mathbb{N}} \in c_{0, \mathcal{U}}(X)$ e $\left(y_{n}\right)_{n \in \mathbb{N}} \in c_{0, \mathcal{U}}(Y)$, isto é, $\left(x_{n}, y_{n}\right)_{n \in \mathbb{N}} \in c_{0, \mathcal{U}}(X \oplus Y)$ se e somente se $\left(\left(x_{n}\right)_{n \in \mathbb{N}},\left(y_{n}\right)_{n \in \mathbb{N}}\right) \in c_{0, \mathcal{U}}(X) \oplus c_{0, \mathcal{U}}(Y)$.

Teorema 4.6.2. Sejam $1<p<q<\infty$ e X uniformemente homeomorfo ao espaço $\ell_{p} \oplus \ell_{q}$. Então para cada ultrafiltro não principal $\mathcal{U}$ em $\mathbb{N}$, temos que $X_{\mathcal{U}}$ é isomorfo a um subespaço complementado de $L_{p} \oplus L_{q}$. Em particular, $X$ também é isomorfo a um subespaço complementado de $L_{p} \oplus L_{q}$.

Demonstração. Segue do Teorema 4.1 .18 que $X_{\mathcal{U}}$ é Lipschitz isomorfo ao espaço $\left(\ell_{p} \oplus \ell_{q}\right)_{\mathcal{U}}$, que por sua vez é isométrico ao espaço $\left(\ell_{p}\right)_{\mathcal{U}} \oplus\left(\ell_{q}\right) \mathcal{U}$. Como $\ell_{p}$ é um espaço $\mathcal{L}_{p}$, assim será $\left(\ell_{p}\right)_{\mathcal{U}}$ por 2.3.78, logo, pelo Teorema 2.3.77, existe um isomorfismo $T:\left(\ell_{p}\right)_{\mathcal{U}} \rightarrow L_{p}$ tal que $T\left[\left(\ell_{p}\right)_{\mathcal{U}}\right]$ é um subespaço complementado de $L_{p}$. Analogamente, existe um isomorfismo $S:\left(\ell_{q}\right)_{\mathcal{U}} \rightarrow L_{q}$ tal que $S\left[\left(\ell_{q}\right)_{\mathcal{U}}\right]$ é um subespaço complementado de $L_{q}$. Então a função $T \oplus S:\left(\ell_{p}\right)_{\mathcal{U}} \oplus\left(\ell_{q}\right)_{\mathcal{U}} \rightarrow L_{p} \oplus L_{q}$ dada por

$$
T \oplus S(\bar{x}, \bar{y})=(T(\bar{x}), S(\bar{y})) \text { para todo }(\bar{x}, \bar{y}) \in\left(\ell_{p}\right)_{\mathcal{U}} \oplus\left(\ell_{q}\right)_{\mathcal{U}}
$$

é um isomorfismo cuja imagem é um subespaço complementado de $L_{p} \oplus L_{q}$. Seja $Y$ tal imagem. Então $X_{\mathcal{U}}$ é Lipschitz isomorfo a $Y$, que é um espaço reflexivo por ser subespaço de $L_{p} \oplus L_{q}$ e então, por 3.5.13, $X$ é isomorfo a um subespaço complementado de $Y$, que por sua vez é um subespaço complementado de $L_{p} \oplus L_{q}$, e segue o resultado.

Definição 4.6.3. Sejam $X$ e $Y$ espaços de Banach de dimensão infinita. Dizemos que $X$ e $Y$ são totalmente incomparáveis se para todo subespaço de dimensão infinita $E$ de $X$ e todo subespaço de dimensão infinita $F$ de $Y$, temos que $E$ e $F$ não são isomorfos.

\section{Exemplo 4.6.4.}

1. Se $1 \leq p<q<\infty$, então $\ell_{p}$ e $\ell_{q}$ são totalmente incomparáveis. Ver [AK98] Corolário 2.1.6, página 32.

2. Se $1<p<2<q<\infty$, então $L_{p}$ e $\ell_{q}$ são totalmente incomparáveis. De fato, segue de [AK98] Proposição 2.2.1, página 33 e [AK98] Teorema 6.4.19, página 160.

Teorema 4.6.5 (Edelstein e Wojtaszczyk). Sejam $X$ e $Y$ espaços de Banach totalmente incomparáveis e $Z$ um subespaço complementado de $X \oplus Y$. Então existe um isomorfismo sobrejetor $\varphi: X \oplus Y \rightarrow X \oplus Y$ tal que $\varphi(Z)=(\varphi(Z) \cap X) \oplus(\varphi(Z) \cap Y)$.

Demonstração. Ver [EW76]. 
Ou seja, o teorema acima nos diz que um subespaço complementado da soma é isomorfo a uma soma de subespaços complementados.

Teorema 4.6.6 (Johnson). Sejam X um espaço de Banach sem cópias do $\ell_{2}$ e $2<p<\infty$. Então todo operador $T: X \rightarrow L_{p}$ se decompõe como $B \circ A$, onde $A: X \rightarrow \ell_{p}$ e $B: \ell_{p} \rightarrow L_{p}$.

Demonstração. Ver [Joh76].

Lema 4.6.7. Sejam $X$ e $Y$ espaços de Banach e $T: X \rightarrow Y$ um isomorfismo tal que $T[X]$ é um subespaço complementado de $Y$. Suponha que existam $Z$ um espaço de Banach, $T_{1}: Z \rightarrow Y$ e $T_{2}: X \rightarrow Z$ operadores contínuos tais que $T=T_{1} \circ T_{2}$. Então $T_{2}$ é um isomorfismo e $T_{2}[X]$ é um subespaço complementado de $Z$.

Demonstração. Sejam $T^{-1}: T[X] \rightarrow X$ a inversa de $T$ e $P: Y \rightarrow T[X]$ uma projeção linear e contínua. Seja $P^{\prime}=T_{2} \circ T^{-1} \circ P \circ T_{1}: Z \rightarrow T_{2}[X]$. $P^{\prime}$ é contínua, linear e além disso temos para cada $x \in X$

$$
P^{\prime}\left(T_{2}(x)\right)=T_{2} \circ T^{-1} \circ P \circ T_{1}\left(T_{2}(x)\right)=T_{2} \circ T^{-1} \circ P(T(x))=T_{2} \circ T^{-1}(T(x))=T_{2}(x) .
$$

Assim, $P^{\prime}$ é uma projeção linear e contínua. Por fim, a função $S=T^{-1} \circ T_{1}: T_{2}[X] \rightarrow X$ é a inversa de $T_{2}$.

Teorema 4.6.8. Sejam $1<p<q<2$ ou $2<p<q<\infty$. Então todo espaço de Banach $X$ uniformemente homeomorfo ao espaço $\ell_{p} \oplus \ell_{q}$ é linearmente isomorfo a $\ell_{p} \oplus \ell_{q}$.

Demonstração. Suponha $2<p<q$. Seja $J: X \rightarrow L_{p} \oplus L_{q}$ um isomorfismo de $X$ em um subespaço complementado de $L_{p} \oplus L_{q}$, que existe pelo Teorema 4.6.2. Sejam também $S_{p}$ e $S_{q}$ as projeções naturais de $L_{p} \oplus L_{q}$ em $L_{p}$ e $L_{q}$, respectivamente.

Como o espaço $\ell_{p} \oplus \ell_{q}$ tem tipo 2, segue que $X$ tem tipo 2 e pelo Teorema de Maurey 4.5.16 toda cópia de $\ell_{2}$ em $X$ é complementada. Mas isto é uma contradição com o Teorema 4.5.11 e $\operatorname{logo} X$ não tem cópias de $\ell_{2}$.

Pelo Teorema de Johnson 4.6.6, os operadores $S_{p} \circ J: X \rightarrow L_{p}$ e $S_{q} \circ J: X \rightarrow L_{q}$ se decompõem como $B_{p} \circ A_{p}$ e $B_{q} \circ A_{q}$, respectivamente, onde $A_{p}: X \rightarrow \ell_{p}, A_{q}: X \rightarrow \ell_{q}$, $B_{p}: \ell_{p} \rightarrow L_{p}$ e $B_{q}: \ell_{q} \rightarrow L_{q}$. Segue que $J$ se decompõe como $J_{2} \circ J_{1}$, onde $J_{1}: X \rightarrow \ell_{p} \oplus \ell_{q}$ é dada por

$$
J_{1}(x)=\left(A_{p}(x), A_{q}(x)\right) \text { para cada } x \in X,
$$

e $J_{2}: \ell_{p} \oplus \ell_{q} \rightarrow L_{p} \oplus L_{q}$ é dada por

$$
J_{2}\left(\left(x_{n}\right)_{n \in \mathbb{N}},\left(y_{n}\right)_{n \in \mathbb{N}}\right)=\left(B_{p}\left(\left(x_{n}\right)_{n \in \mathbb{N}}\right), B_{q}\left(\left(y_{n}\right)_{n \in \mathbb{N}}\right)\right) \text { para cada }\left(\left(x_{n}\right)_{n \in \mathbb{N}},\left(y_{n}\right)_{n \in \mathbb{N}}\right) \in \ell_{p} \oplus \ell_{q}
$$

Portanto, segue de 4.6.7 que $J_{1}$ é um isomorfismo de $X$ em um subespaço complementado de $\ell_{p} \oplus \ell_{q}$. Mas pelo Teorema de Edelstein 4.6.5, $X$ é isomorfo a $E \oplus F$, onde $E$ é um subespaço complementado de $\ell_{p}$ e $F$ um subespaço complementado de $\ell_{q}$. Ou seja, $E$ só pode ser $\ell_{p}$ 
ou um subespaço de dimensão finita de $\ell_{p}$ e $F$ só pode ser $\ell_{q}$ ou um subespaço de dimensão finita de $\ell_{q}$. Segue então do Teorema 4.5.20 que $X$ é isomorfo a $\ell_{p} \oplus \ell_{q}$.

Caso $1<p<q<2$, temos que $X^{*}$ é uniformemente homeomorfo ao espaço $\ell_{p^{*}} \oplus \ell_{q^{*}}$, onde $p^{*}$ e $q^{*}$ são os conjugados de $p$ e $q$, respectivamente. Pelo que vimos acima, $X^{*}$ é isomorfo ao $\ell_{p^{*}} \oplus \ell_{q^{*}}$. Como $X$ é superreflexivo, é em particular reflexivo e então $X$ é isomorfo ao $\ell_{q} \oplus \ell_{q}$

Para o caso em que $1<p<2<q<\infty$, precisamos usar as técnicas com grafos métricos que foram estudadas anteriormente.

Teorema 4.6.9. Sejam $1 \leq p_{1}<p_{2}<\ldots<p_{n}<\infty$. Se $r \notin\left\{p_{1}, \ldots, p_{n}\right\}$, então nãa existe mergulho grosseiro Lipschitz de $\ell_{r}$ em $\ell_{p_{1}} \oplus \ldots \oplus \ell_{p_{n}}$.

Demonstração. Suponha por contradição que exista $f: \ell_{r} \rightarrow \ell_{p_{1}} \oplus \ldots \oplus \ell_{p_{n}}$ mergulho grosseiro Lipschitz e consideremos primeiro o caso em que $p_{m}<r<p_{m+1}$ para algum $m<n$. Sejam $X=\left(\ell_{p_{1}} \oplus \ldots \oplus \ell_{p_{m}}\right)_{\ell_{p_{m}}}$ e $Y=\left(\ell_{p_{m+1}} \oplus \ldots \oplus \ell_{p_{n}}\right)_{\ell_{\infty}}$. Podemos assumir então sem perda de generalidade que $f: \ell_{r} \rightarrow X \oplus_{\infty} Y$, é da forma $f=(g, h)$ e que satisfaz:

$$
\|x-y\| \leq\|f(x)-f(y)\| \leq C\|x-y\|,\|x-y\| \geq 1 .
$$

Sejam $k \in \mathbb{N}$ e $\delta>0$ tais que $3.2^{1 / r}\left(C k^{1 / p_{m+1}-1 / r}+\delta\right)<1$. Podemos aplicar a Proposição 4.5.9 para $g$ e obtermos $\tau>k, x \in \ell_{r}, K \subset X$ compacto e $N \in \mathbb{N}$ tais que $g\left[x+\tau B_{E_{N}}\right] \subset K+\delta \tau B_{X}$. Definimos $\mathbb{M}=\{n \in \mathbb{N}: n>N\}$ e $\varphi: G_{k}(\mathbb{M}) \rightarrow \ell_{r}$ por

$$
\varphi\left(n_{1}, \ldots, n_{k}\right)=x+\tau k^{-1 / r}\left(e_{n_{1}}+\ldots+e_{n_{k}}\right) \in x+\tau B_{E} .
$$

Então $g \circ \varphi\left[G_{k}(\mathbb{M})\right] \subset K+\delta \tau B_{X}$ e pelo Lema 4.4.5 existe $M_{0} \subset \mathbb{M}$ infinito tal que

$$
\operatorname{diam}\left(g \circ \varphi\left[G_{k}\left(M_{0}\right)\right]\right) \leq 3 \delta \tau
$$

Por outro lado, $\|h(\varphi(\bar{n}))-h(\varphi(\bar{m}))\| \leq C\|\varphi(\bar{n})-\varphi(\bar{m})\|$ e para $\bar{n}, \bar{m} \in G_{k}\left(M_{0}\right)$ distintos,

$$
1<2^{1 / r} k^{1-1 / r} \leq 2^{1 / r} \tau k^{-1 / r} \leq\|\varphi(\bar{n})-\varphi(\bar{m})\| \leq 2^{1 / r} \tau k^{-1 / r} d(\bar{n}, \bar{m})^{1 / r} \leq 2^{1 / r} \tau k^{-1 / r} d(\bar{n}, \bar{m}) .
$$

Isto mostra que $\operatorname{Lip}(h \circ \varphi) \leq 2^{1 / r} C \tau k^{-1 / r}$ e aplicando o Teorema 4.4.7, obtemos $\mathbb{M}^{\prime} \subset M_{0}$ infinito tal que $\operatorname{diam}\left(h \circ \varphi\left[G_{k}\left(\mathbb{M}^{\prime}\right)\right]\right) \leq 3.2^{1 / r} C \tau k^{1 / p_{m+1}-1 / r}$. Segue então que

$$
\operatorname{diam}\left(f \circ \varphi\left[G_{k}\left(\mathbb{M}^{\prime}\right)\right]\right) \leq 3.2^{1 / r} \tau\left(C k^{1 / p_{m+1}-1 / r}+\delta\right)
$$

Por outro lado, sejam $\bar{n}=\left(n_{1}, \ldots, n_{k}\right), \bar{m}=\left(m_{1}, \ldots, m_{k}\right) \in G_{k}\left(\mathbb{M}^{\prime}\right)$ tais que $n_{i} \neq m_{i}$ para cada $i \in\{1, \ldots, k\}$. Temos

$$
\operatorname{diam}\left(\varphi\left[G_{k}\left(\mathbb{M}^{\prime}\right)\right]\right) \geq\|\varphi(\bar{n})-\varphi(\bar{m})\|=(2 k)^{1 / r} \tau k^{-1 / r}=2^{1 / r} \tau>\tau
$$


e então $\operatorname{diam}\left(f \circ \varphi\left[G_{k}\left(\mathbb{M}^{\prime}\right)\right]\right)>\tau$. Segue por fim que $1<3.2^{1 / r}\left(C k^{1 / p_{m+1}-1 / r}+\delta\right)$, contradizendo a escolha de $k$ e $\delta$.

No caso em que $r>p_{n}$, a demonstração é exatamente a mesma que fizemos em 4.5.13.

Teorema 4.6.10 (Kalton, Randrianarivony). Sejam $1<p<2<q<\infty$. Então todo espaço de Banach $X$ uniformemente homeomorfo a $\ell_{p} \oplus \ell_{q}$ é também linearmente isomorfo a $\ell_{p} \oplus \ell_{q}$.

Demonstração. Pelo Teorema 4.6.2 existe um mergulho $J: X \rightarrow L_{p} \oplus L_{q}$ cuja imagem é um subespaço complementado. Vamos escrever $J=\left(J_{p}, J_{q}\right)$, onde $J_{p}: X \rightarrow L_{p}$ e $J_{q}: X \rightarrow L_{q}$. Pelo teorema acima, $\ell_{2}$ não pode ser mergulhado em $X$. Como $q>2$, segue do Teorema de Johnson 4.6.6 que $J_{q}=A \circ B$, onde $B: X \rightarrow \ell_{q}$ e $A: \ell_{q} \rightarrow L_{q}$. Sejam $J_{1}: L_{p} \oplus \ell_{q} \rightarrow L_{p} \oplus L_{q}$ dado por $J_{1}(x, y)=(x, A(y))$ para cada $(x, y) \in L_{p} \oplus \ell_{q}$ e $J_{2}: X \rightarrow L_{p} \oplus \ell_{q}$ dado por $J_{2}(x)=\left(J_{p}(x), B(x)\right)$ para cada $x \in X$. Temos então que $J=J_{1} \circ J_{2}$ e por $4.6 .7 X$ será isomorfo a um subespaço complementado de $L_{p} \oplus \ell_{q}$. Segue do Teorema de Edelstein 4.6.5 que $X$ será isomorfo a $E \oplus F$, onde $E$ é um subespaço complementado de $L_{p}$ e $F$ um subespaço complementado de $\ell_{q}$. Veja que $E$ não pode conter cópias de $\ell_{2}$ e pelo Teorema de Johnson 4.5.19, $E$ será de dimensão finita ou o $\ell_{p}$. Também temos que $F$ só pode ser $\ell_{q}$ ou um subespaço de dimensão finita. Se qualquer um dos dois fosse de dimensão finita teríamos um homeomorfismo uniforme entre $\ell_{p} \oplus \ell_{q}$ e $\ell_{p}$ ou $\ell_{q}$, contradizendo o Teorema 4.5.20. Logo $X$ é isomorfo a $\ell_{p} \oplus \ell_{q}$. 


\section{Capítulo 5}

\section{Problemas em AberTo}

Neste capítulo, vamos comentar brevemente sobre alguns problemas em aberto que estão relacionados ao que estudamos nos capítulos passados. Como referência aos problemas em aberto indicamos [GLZ14], especificamente a tabela 1 da página 3.

Vimos em 4.5.20 que todo espaço de Banach $X$ uniformemente homeomorfo a $\ell_{p}$ é também linearmente isomorfo a $\ell_{p}$ para cada $1<p<\infty$. Entretanto, no caso em que $p=1$, temos o seguinte problema.

Problema 1 Se $X$ é Lipschitz isomorfo ao $\ell_{1}, X$ é linearmente isomorfo a $\ell_{1}$ ?

Um resultado positivo sobre $\ell_{1}$ pode ser obtido ao considerarmos espaços duais e repetir a demonstração do Teorema 3.5.13, trocando a topologia fraca pela topologia fraca*. Ver [Kal08] Teorema 2.1.17 para detalhes da demonstração.

Teorema 5.0.1. Seja X um espaço dual Lipschitz isomorfo a $\ell_{1}$. Então X é linearmente isomorfo a $\ell_{1}$.

Quando consideramos o espaço $c_{0}$, não temos a propriedade de Radon-Nikodým para podermos linearizar mergulhos Lipschitz. Entretanto, em [GKL00] é provado o seguinte resultado, onde aplica-se também o princípio de Gorelik.

Teorema 5.0.2 (Godefroy, Kalton e Lancien). Seja X um espaço Lipschitz isomorfo a $c_{0}$. Então $X$ é linearmente isomorfo a $c_{0}$.

Entretanto, está em aberto o seguinte problema.

Problema 2 Se $X$ é uniformemente homeomorfo ao $c_{0}, X$ é linearmente isomorfo a $c_{0}$ ?

Vimos em 3.5.18 que para cada $1<p<\infty$ todo espaço de Banach Lipschitz isomorfo a $L_{p}$ é também linearmente isomorfo a $L_{p}$. No caso da estrutura uniforme, temos o seguinte problema. 
Problema 3 Se $1<p<\infty, p \neq 2$ e $X$ é uniformemente homeomorfo ao $L_{p}, X$ é linearmente isomorfo a $L_{p}$ ?

Por fim, observamos que para demonstrar os Teoremas 4.6.8 e 4.6.10 foi necessário provar que o espaço de Banach $X$ uniformemente isomorfo a $\ell_{p} \oplus \ell_{2}$ não contenha cópias de $\ell_{2}$. Assim, não podemos aplicar essas técnicas para o espaço $\ell_{p} \oplus \ell_{2}$.

Problema 4 Se $1<p<\infty, p \neq 2$ e $X$ é uniformemente homeomorfo ao $\ell_{p} \oplus \ell_{2}, X$ é linearmente isomorfo a $\ell_{p} \oplus \ell_{2}$ ?

Mas temos um resultado positivo sobre isomorfismos Lipschitz.

Teorema 5.0.3. Seja $X$ um espaço de Banach Lipschitz isomorfo a $\ell_{p} \oplus \ell_{2}$. Então $X$ é linearmente isomorfo a $\ell_{p} \oplus \ell_{2}$.

Demonstração. Como $\ell_{p} \oplus \ell_{2}$ é reflexivo, podemos aplicar o teorema 3.5.13 para obtermos que $X$ é isomorfo a um subespaço complementado de $\ell_{p} \oplus \ell_{2}$. Pelo Teorema 4.6.5, $X$ é isomorfo a $E \oplus F$, onde $E$ é um subespaço complementado de $\ell_{p}$ e $F$ um subespaço complementado de $\ell_{2}$. Ou seja, $E$ só pode ser $\ell_{p}$ ou um subespaço de dimensão finita de $\ell_{p}$ e $F$ só pode ser $\ell_{2}$ ou um subespaço de dimensão finita de $\ell_{2}$. Segue então do Teorema 4.5.20 que $E=\ell_{p}$ e $F=\ell_{2}$. 


\section{Apêndice A}

\section{TEOREMA DE MAZUR ULAM}

Proposição A.0.1. Sejam $X, Y$ espaços de Banach reais e $f: X \rightarrow Y$ uma função contínua. São equivalentes:

1. $f((x+y) / 2)=(f(x)+f(y)) / 2$ para todos $x, y \in X$.

2. $f(t x+(1-t) y)=t f(x)+(1-t) f(y)$ para todos $x, y \in X$ e para cada $t \in[0,1]$.

3. $f(x)=T(x)+f(0)$ para cada $x \in X$, onde $T \in \mathcal{L}(X, Y)$.

Demonstração. 1) $\Rightarrow 2)$ Vamos mostrar primeiro que $f\left(\left(x_{1}+\ldots+x_{k}\right) / k\right)=\left(f\left(x_{1}\right)+\ldots+\right.$ $\left.f\left(x_{k}\right)\right) / k$ para quaisquer $x_{1}, \ldots, x_{k} \in X$. Se $k=2^{n}$, então

$f\left(x_{1}+\ldots+x_{2^{n}}\right)=\left(f\left(x_{1}+\ldots+x_{2^{n-1}}\right)+f\left(x_{2^{n-1}+1}+\ldots+x_{2^{n}}\right)\right) / 2=\ldots=\left(f\left(x_{1}\right)+\ldots+f\left(x_{2^{n}}\right)\right) / 2^{n}$

Se $2^{n-1}<k<2^{n}$, seja $\bar{x}=\left(x_{1}+\ldots+x_{k}\right) / k$. Então

$f(\bar{x})=f\left(2^{n} \bar{x} / 2^{n}\right)=f\left(\left(x_{1}+\ldots x_{k}+\left(2^{n}-k\right) \bar{x}\right) / 2^{n}\right)=\left(f\left(x_{1}\right)+\ldots f\left(x_{k}\right)\right) / 2^{n}+\left(2^{n}-k\right) f(\bar{x}) / 2^{n}$.

Segue dai que $k f(\bar{x})=f\left(x_{1}\right)+\ldots f\left(x_{k}\right)$. Por fim, se $\frac{m}{n} \in[0,1]$ com $m, n \in \mathbb{Z}$, temos que

$f((m / n) x+(1-(m / n) y))=f((x+\ldots+x+y+\ldots+y) / n)=(m f(x)+(n-m) f(y)) / n$,

onde acima somamos $x m$ vezes e $y n-m$ vezes. A implicação para $t \in[0,1]$ segue da continuidade da $f$ e da densidade dos racionais.

$2) \Rightarrow 3)$ Definimos $T: X \rightarrow Y$ por $T(x)=f(x)-f(0)$. Obviamente $T$ é contínua. Vamos mostrar que $T$ é linear. Veja que para $n \in \mathbb{N}, f(x)=f(n x / n)=f(n x) / n+(n-1) f(0) / n$ e $\operatorname{assim} f(n x)=n f(x)-(n-1) f(0)$. Assim,

$$
T(n x)=f(n x)-f(0)=n(f(x)-f(0))=n T(x) .
$$


Se $x, y \in X$, então

$$
\begin{aligned}
T(x+y)=f(x+y)-f(0)=(f(2 x)+f(2 y)) / 2-f(0) & =(2 f(x)+2 f(y)-2 f(0)) / 2-f(0) \\
& =f(x)-f(0)+f(y)-f(0) \\
& =T(x)+T(y) .
\end{aligned}
$$

$\operatorname{Logo} T(x)+T(-x)=T(0)=0$ e $T(n x)=n T(x)$ para todo $x \in X$ e todo $n \in \mathbb{Z}$. Então $T(x)=n T(x / n)$, nos dando $T(x / n)=T(x) / n$ e $T(q x)=q T(x)$ para todo $x \in X$ e todo $q \in \mathbb{Q}$. O resultado segue da continuidade da $f$.

Definição A.0.2. Sejam $X, Y$ espaços de Banach reais e $f: X \rightarrow Y$ uma função. Dizemos que $f$ é uma função afim se satisfaz uma das três propriedades acima.

Teorema A.0.3 (Mazur-Ulam). Sejam $X, Y$ espaços de Banach reais e $f: X \rightarrow Y$ uma isometria sobrejetora de $X$ em $Y$. Então $f$ é uma função afim.

Demonstração. Sejam $x, y \in X$. Definimos $K_{0}(x, y)=\{u \in X:\|u-x\|=\|u-y\|=\| x-$ $y \| / 2\}$ e para $n \geq 1$, definimos $K_{n}(x, y)=\left\{u \in K_{n-1}(x, y): K_{n-1}(x, y) \subset B\left(u ; d_{n-1} / 2\right)\right\}$, onde $d_{n}=\operatorname{diam}\left(K_{n}\right)$ para cada $n \geq 0$. Note que cada $K_{n}(x,-x)$ é simétrico. Afirmamos que $\bigcap_{n \geq 0} K_{n}(x,-x)=\{0\}$. De fato, $0 \in K_{0}(x,-x)$ e se $0 \in K_{n}(x,-x)$, a simetria nos dá $\|u\|<$ $d_{n} / 2$ para todo $u \in K_{n}(x,-x)$ e asssim $K_{n}(x,-x) \subset B\left(0 ; d_{n} / 2\right)$, isto é, $0 \in K_{n+1}(x,-x)$ e por indução $\{0\} \subset \bigcap_{n \geq 0} K_{n}(x,-x)$. Por outro lado, se $u, v \in K_{n+1}(x,-x)$, temos que $v \in K_{n}(x,-x) \subset B\left(u ; \bar{d}_{n} / 2\right)$, nos dando $\|u-v\|<d_{n} / 2$ e $d_{n+1} \leq d_{n} / 2 \leq \ldots \leq d_{0} / 2^{n}$. Portanto $\bigcap_{n \geq 0} K_{n}(x,-x) \subset\{0\}$.

Em geral, $\{(x+y) / 2\}=\{0\}+(x+y) / 2=\bigcap_{n \geq 0} K_{n}((x-y) / 2,(y-x) / 2)+(x+y) / 2=$ $\bigcap_{n \geq 0} K_{n}(x, y)$. Vamos mostrar por indução que $K_{n}(f(x), f(y)) \subset f\left[K_{n}(x, y)\right]$ para cada $n \geq 0$. De fato, como $f$ é isometria sobrejetora, $f\left[K_{0}(x, y)\right]=K_{0}(f(x), f(y))$. Suponha $K_{n}(f(x), f(y)) \subset$ $f\left[K_{n}(x, y)\right]$, então para cada $u \in K_{n+1}, K_{n}(f(x), f(y)) \subset f\left[K_{n}(x, y)\right] \subset f\left[B\left(u ; d_{n} / 2\right)\right]=$ $B\left(f(u) ; d_{n} / 2\right)$, isto é, $f(u) \in K_{n+1}(f(x), f(y))$.

Podemos provar que $f^{-1}$ satisfaz a mesma inclusão, isto é, $K_{n}(x, y) \subset f^{-1}\left[K_{n}(f(x), f(y))\right]$, o que implica $f\left[K_{n}(x, y)\right] \subset K_{n}(f(x), f(y))$ para cada $n \geq 0$ e a igualdade segue. Finalmente,

$$
\{(f(x)+f(y)) / 2\}=\bigcap_{n \geq 0} K_{n}(f(x), f(y))=\bigcap_{n \geq 0} f\left[K_{n}(x, y)\right]=f\left[\bigcap_{n \geq 0} K_{n}(x, y)\right]=\{f((x+y) / 2)\} .
$$




\section{Referências Bibliográficas}

[AK98] F. Albiac e N. J. Kalton. Topics in Banach Space Theory. Springer, 2 edição, 1998. $24,26,28,67,96$

[BL00] Y. Benyamini e J. Lindenstrauss. Geometric Nonlinear Functional Analysis. American Mathematical Society Colloquium Publications, vol. 48, American Mathematical Society, 1 edição, 2000. 31, 44

[Bou83] J. Bourgain. Remarks on the extension of Lipschitz maps defined on discrete sets and uniform homeomorphisms. Ark. Mat., 21:163-168, 1983. 29, 80, 93

[Dur13] R. Durrett. Probability: Theory and Examples. Perspectiva, 4.1 edição, 2013. 3, 8, 12,13

[EG92] L.C. Evans e R.F. Gariepy. Measure theory and fine properties of functions. CRC Press, 1 edição, 1992. 31

[Enf70] P. Enflo. Uniform structures and square roots in topological groups, II. Israel J. Math., 8:253-272, 1970. 76

[EW76] I. S. Edelstein e P. Wojtaszczyk. On projections and unconditional bases in direct sums of Banach spaces. Studia Math, 56:263-276, 1976. 96

[GKL00] G. Godefroy, N. J. Kalton e G. Lancien. Subspaces of $c_{0}(\mathbb{N})$ and Lipschitz isomorphisms. Geom. Funct. Anal., 10:798-820, 2000. 101

[GLZ14] G. Godefroy, G. Lancien e V. Zizler. The non-linear geometry of Banach spaces after Nigel Kalton. Rocky Mountain J. Math, 44:1529-1583, 2014. 101

[Gor94] E. Gorelik. The uniform nonequivalence of $L_{p}$ and $\ell_{p}$. Israel J. Math., 87:1-8, 1994. 80

[Haa33] A. Haar. Der Massbegriff in der Theorie der kontinuierlichen Gruppen. Annals of Mathematics, 34:147-169, 1933. 32

[HM82] S. Heinrich e P. Mankiewicz. Applications of ultrapowers to the uniform and Lipschitz classification of Banach spaces. Studia Math., 73:225-251, 1982. 2, 75, 77, 94

[JL69] H. P. Rosenthal J. Lindenstrauss. The $\mathcal{L}_{p}$ Spaces. Israel J Math, 1 edição, 1969. 29

[JLS96] W. B. Johnson, J. Lindenstrauss e G. Schechtman. Banach spaces determined by their uniform structures. Geom. Funct. Anal., 6:430-470, 1996. 2, 71, 95 
[JO74] W. B. Johnson e E. Odell. Subspaces of $L_{p}$ which embed into $\ell_{p}$. Compositio Math., 28:37-49, 1974. 94

[Joh76] W. B. Johnson. Operators into $L_{p}$ which factor through $\ell_{p}$. J. London Math. Soc., 14:333-339, 1976. 97

[Kad67] M. I. Kadets. A proof of the topological equivalence of all separable infinitedimensional Banach spaces. Funkcional. Anal., 1:61-70, 1967. 1

[Kal08] N. J. Kalton. The Nonlinear Geometry of Banach Spaces. Rev. Mat. Complut., 21:7-60, 2008. 2, 31, 71, 101

[KR08] N. J. Kalton e N. L. Randrianarivony. The coarse Lipschitz geometry of $\ell_{p} \oplus \ell_{q}$. Math. Ann., 341:223-237, 2008. 71, 81, 95

[Mau74] B. Maurey. Un theorème de prolongement. C. R. Acad. Paris, 279:329-332, 1974. 94

[Meg98] R. E. Megginson. An Introduction to Banach Space Theory. Springer, 1 edição, 1998. 14, 15, 16, 17, 18, 19, 20

[Mic56] E. Michael. Continuous selections. Annals Math, 63:361-382, 1956. 18

[Nie97] O. A. Nielsen. An introduction to integration and measure theory. WileyInterscience, 1 edição, 1997. 45

[Pro56] Yu.V. Prokhorov. Convergence of random processes and limit theorems in probability theory. Theory Probab. Appl., 1:157-214, 1956. 35

[Rib76] M. Ribe. On uniformly homeomorphic normed spaces. Ark. Mat., 14:237-244, 1976. 77

[Rib78] M. Ribe. On uniformly homeomorphic normed spaces, II. Ark. Mat., 1:1-9, 1978. 94

[Rud70] W. Rudin. Real and Complex. Perspectiva, 22º edição, 1970. 3, 4, 5, 6, 7

[Sch30] J. Schauder. Der Fixpunktsatz in Funktionalräumen. Studia Math, 2:171-180, 1930. 18

[Spi65] M. Spivak. Calculus on Manifolds. Perspectiva, 22º edição, 1965. 6

[Ste78] J. Stern. Ultrapowers and local properties of Banach spaces. Trans. Amer. Math. Soc., 240:231-252, 1978. 60

[Tor81] H. Toruńczyk. Characterizing Hilbert space topology. Fund. Math., 111:247-262, 1981. 1 


\section{Índice Remissivo}

\section{Símbolos}

$B\left(x_{0} ; r\right), 14$

$B_{X}, 14$

$C_{b}(X), 33$

$G_{k}(\mathbb{M}), 82$

$K_{f}(t), 72$

$L_{p}(\mu), 5$

$\operatorname{Lip}_{0}(f), 64$

$\operatorname{Mid}(x, y, \delta), 78$

$\operatorname{Pr}(X), 34$

$S_{X}, 14$

$X$-medida, 43

$X / Y, 15$

$\ell_{\infty}(G), 62$

$\ell_{\infty}^{n}, 26$

$\ell_{p}^{n}, 26$

$\lambda$-sistema, 3

$\mathbb{E}[\xi], 9$

$\omega_{f}(t), 71$

$\pi$-sistema, 3

\section{C}

Conjunto Haar nulo, 32

Convolução, 35, 50

Cotipo, 25

D

Distância de Banach-Mazur, 26

E

Espaço de Probabildiade, 8

Espaço vetorial topológico, 16

F

Função absolutamente contínua, 45

Função afim, 63

Função Bochner integrável, 39

Função com suporte compacto, 49

Função fraca*-diferenciável, 56

Função grosseira Lipschitz contínua, 72

Função grosseiramente contínua, 72
Função Gâteaux diferenciável, 44

Função lipschitziana, 31

Função mensurável, 38

$\mathbf{H}$

Homeomorfismo uniforme, 71

I

Integral de Bochner, 40

Isomorfismo Lipschitz, 31

M

Mergulho Lipschitz, 55

$\mathbf{P}$

Ponto de Lebesgue, 43

Propriedade de Radon-Nikodým, 44

$\mathbf{R}$

Rede, 19

Representabilidade finita, 26

$\mathrm{S}$

Sequência de Rademacher, 24

Subespaço complementado, 16

Superreflexividade, 28

$\mathbf{T}$

Tipo, 25

Topologia fraca, 17

Topologia fraca*, 17

U

Ultrafiltro, 20

Ultralimite, 21

Ultraproduto, 23

V

Variáveis aleatórias independentes, 10

Vetor aleatório, 8, 32 